\title{
CONTROLLING THE PROPAGATION OF LIGHT IN DISORDERED SCATTERING MEDIA
}


Promotiecommissie

Promotor

Assistent Promotor

prof. dr. A. Lagendijk

dr. A. P. Mosk

Overige leden

prof. dr. D. Lohse

prof. dr. J. P. Woerdman

prof. dr. W. L. Vos

prof. dr. A. C. Boccara

Paranimfen

H. E. Holland

M. Pil

The work described in this thesis is part of the research program of the

'Stichting voor Fundamenteel Onderzoek der Materie (FOM)', which is financially supported by the

'Nederlandse Organisatie voor Wetenschappelijk Onderzoek' (NWO)'.

This work was carried out at the

Complex Photonic Systems Group,

Department of Science and Technology

and $\mathrm{MESA}^{+}$Institute for Nanotechnology,

University of Twente, P.O. Box 217,

7500 AE Enschede, The Netherlands.

This thesis can be downloaded from

http://www.wavesincomplexmedia.com.

ISBN: 978-90-365-2663-0 


\title{
CONTROLLING THE PROPAGATION OF LIGHT IN DISORDERED SCATTERING MEDIA
}

\section{PROEFSCHRIFT}

\author{
ter verkrijging van \\ de graad van doctor aan de Universiteit Twente, \\ op gezag van de rector magnificus, \\ prof. dr. W.H.M. Zijm, \\ volgens besluit van het College voor Promoties \\ in het openbaar te verdedigen \\ op donderdag 24 april 2008 om 16.45 uur
}

door

Ivo Micha Vellekoop

geboren op 11 november 1977

te 's-Gravenhage 
Dit proefschrift is goedgekeurd door:

prof. dr. A. Lagendijk en dr. A. P. Mosk 
“... no more substance than a pattern formed by frost that a simple rise in temperature would reduce to nothing."

“... pas plus de consistance qu'un motifformé par le givre qu'un simple redoux suffit à anéantir."

- Michel Houellebecq, La Possibilité d'une île 



\section{Contents}

1 Introduction 11

1.1 Opaque lenses . . . . . . . . . . . . . . . . . . . . 12

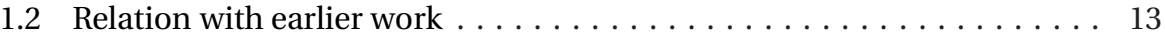

1.3 Mathematical tools for analyzing complex system . . . . . . . . . . 14

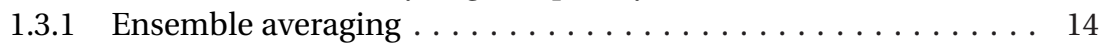

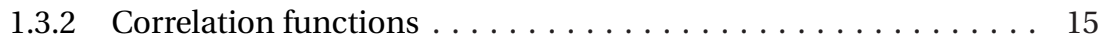

1.3.3 Probability density functions $\ldots \ldots \ldots \ldots \ldots \ldots \ldots \ldots$

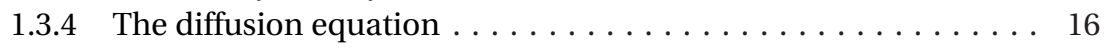

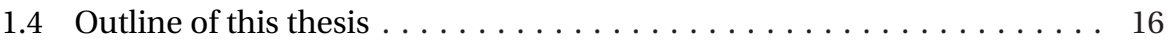

2 Experimental apparatus 19

2.1 Wavefront synthesizer . . . . . . . . . . . . . . . . . 20

2.1.1 Principle of a twisted nematic liquid crystal display . . . . . . . 20

2.1.2 Liquid crystal display characterization . . . . . . . . . 21

2.1.3 Liquid crystal phase-mostly modulation . . . . . . . . . 23

2.1.4 Decoupled amplitude and phase modulation . . . . . . . . . 24

2.1.5 Demonstration of amplitude and phase modulation . . . . . . 26

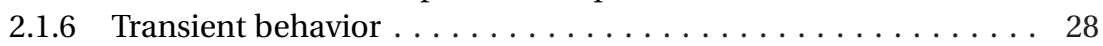

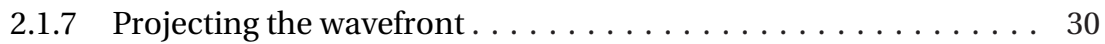

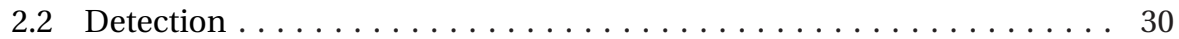

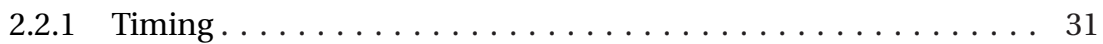

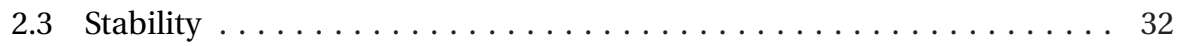

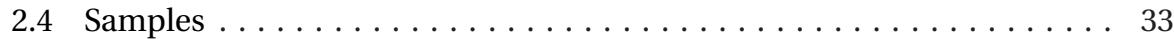

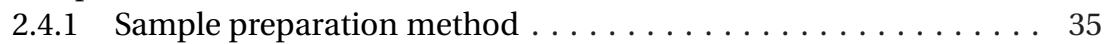

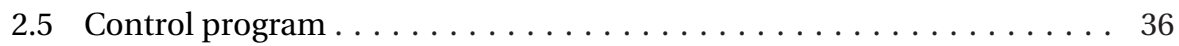

2.5.1 LabView and $\mathrm{C}++$ mixed programming $\ldots \ldots \ldots 37$

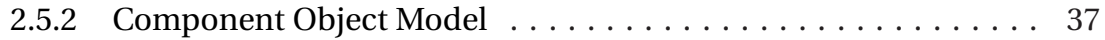

2.5.3 Global structure of the control program . . . . . . . . 38

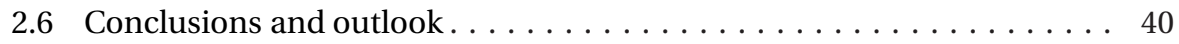

3 Focusing coherent light through opaque strongly scattering media 43

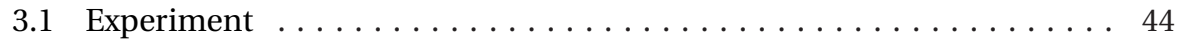

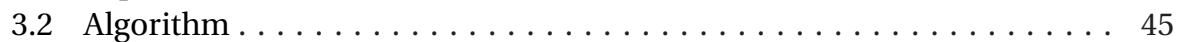

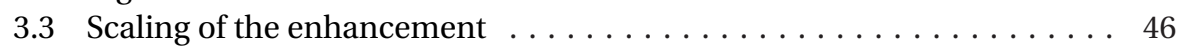

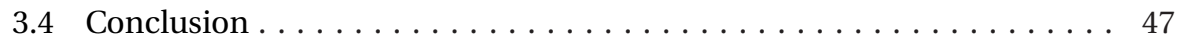

4 The focusing resolution of opaque lenses $\quad 51$

4.1 Wavefront shaping with an opaque lens . . . . . . . . . . . . . 52

4.2 Measured focusing resolution of an opaque lens . . . . . . . . 53

4.3 Measured relation between the focus and the speckle correlation func-

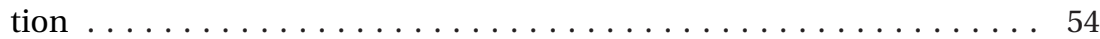


4.4 Continuous field theory for opaque lenses $\ldots \ldots \ldots \ldots \ldots \ldots$

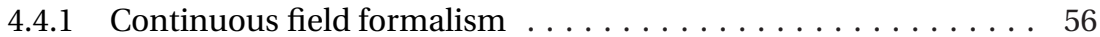

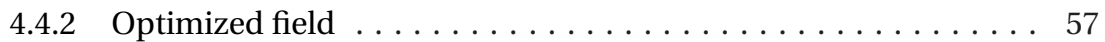

4.4.3 Better than diffraction limited focusing . . . . . . . . 58

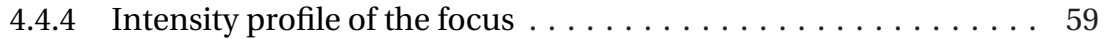

4.4.5 Connection with speckle correlation function . . . . . . . . 60

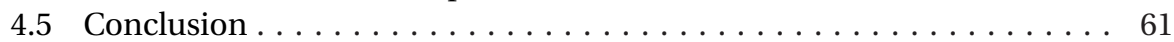

5 Demixing light paths inside disordered metamaterials 63

5. Experimental details . . . . . . . . . . . . . . . . . . . 68

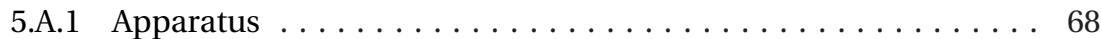

5.A.2 Measurement sequence . . . . . . . . . . . . . . 69

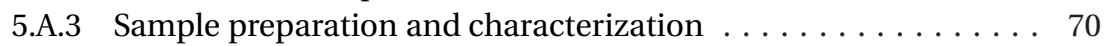

5.A.4 3-dimensional scan results . . . . . . . . . . . . . . . 72

5.B Analysis of the channel demixing method . . . . . . . . . . . 73

5.B.1 Maximum enhancement - scalar waves, simplified case . . . . . . 74

5.B.2 Maximum enhancement - finite size probe . . . . . . . . . . 74

5.B.3 Maximum enhancement of speckle scan . . . . . . . . . . 75

5.B.4 Diffuse and ballistic intensities inside the medium . . . . . . . 75

6 Exploiting the potential of disorder in optical communication $\quad 79$

6.1 Increasing the information density $\ldots \ldots \ldots \ldots \ldots \ldots \ldots \ldots$

6.2 Experimental details . . . . . . . . . . . . . . . . 81

7 Phase control algorithms for focusing light through turbid media 85

7.1 Algorithms for inverse diffusion . . . . . . . . . . . . . . . . . . . . . . 87

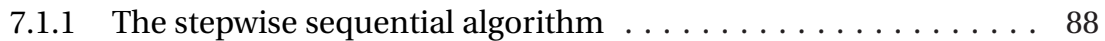

7.1 .2 The continuous sequential algorithm . . . . . . . . . 89

7.1 .3 The partitioning algorithm ............... 89

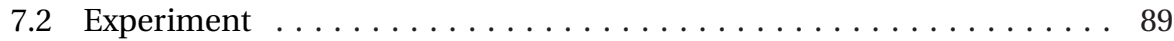

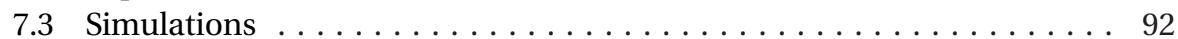

7.4 Analytical expressions for the enhancement . . . . . . . . . . 93

7.4.1 Performance in fluctuating environments . . . . . . . . . 93

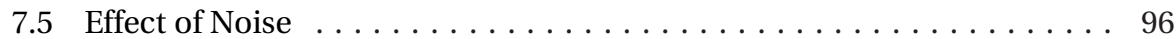

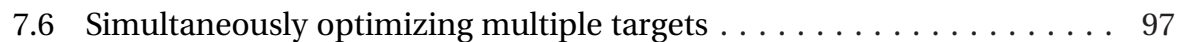

7.7 Conclusion . . . . . . . . . . . . . . . . . . . . . 98

7.A Calculation of the performance of the partitioning algorithm . . . . . 99

8 Transport of light with an optimized wavefront 103

8.1 Random matrix theory . . . . . . . . . . . . . . . . . . . 103

8.1.1 Random matrix theory in a waveguide geometry . . . . . . . . . . . . . . . . 104

8.1.2 Distribution of transmission eigenvalues . . . . . . . . . 106

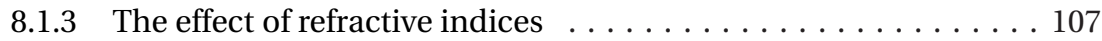

8.2 A new class of experimental observables . . . . . . . . . . . . . . . . 109

8.2.1 Observables in passive measurements . . . . . . . . . . 111

8.2.2 Observables in active measurements . . . . . . . . . . . . 112

8.2.3 Comparison with the uncorrelated model . . . . . . . . . . . . . 114 
8.2.4 Random matrix theory for thin samples . . . . . . . . 115

8.2 .5 Non-diffusive behavior . . . . . . . . . . . . . . . . . . 115

8.3 Random matrix theory in an optical experimental situation . . . . . . 116

8.3 .1 Slab geometry . . . . . . . . . . . . . . . 116

8.3.2 Wavefront modulation imperfections . . . . . . . . . 118

8.3.3 Examples of realistic experimental situations . . . . . . . 119

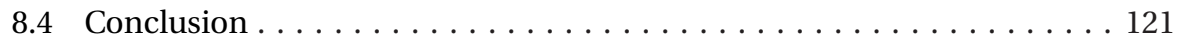

9 Observation of open transport channels in disordered optical systems $\quad 125$

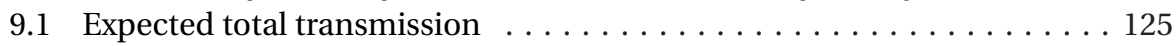

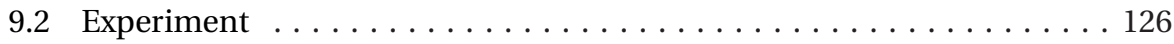

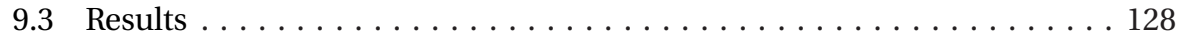

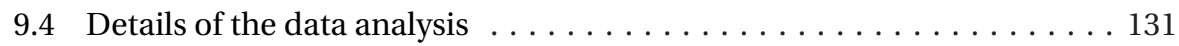

9.4.1 Diffuse transmission measurement with a camera . . . . . . . . 131

9.4.2 Possible causes of systematical error . . . . . . . . . . . . . 132

9.4.3 Measurement of the incident power . . . . . . . . . . . 133

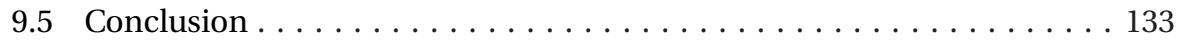

$\begin{array}{ll}10 \text { Summary and outlook } & 135\end{array}$

$\begin{array}{ll}\text { Nederlandse samenvatting } & 137\end{array}$

$\begin{array}{ll}\text { Dankwoord } & 141\end{array}$ 
10 | Contents 


\section{Chapter 1 \\ Introduction}

Devices that use or produce light play an important role in modern life. Among the numerous daily applications of light are displays, telecommunication, data storage, sensors. In industry, medicine and scientific research, optical techniques are also absolutely indispensable. Light is used for imaging and microscopy, but also for detecting and treating diseases, analyzing chemical compounds and investigating living cells on a molecular level.

In a transparent medium, like glass or air, light propagates along a straight line. However, as we all know from daily experience, it is impossible to see through, for instance, white paint or the shell of an egg. Such opaque materials have a microscopic structure that makes it impossible for light to go straight through. Figure. 1.1a shows schematically what happens when a beam of light impinges on a white opaque object: collisions with tiny particles causes the light to spread out and lose all directionality. This process is called diffusion of light; it is comparable to the irreversible diffusion process that makes a drop of ink in a glass of water spread out evenly.

Scattering and diffusion of light are huge limitations for optical imaging, but also severely hinder telecommunication, spectroscopy, and other optical techniques. In the last few decades, a tremendous effort was put in developing imaging methods that work in strongly scattering media.[1] That research has brought forward important new imaging methods like optical coherence tomography[2], diffusion tomography[3] and laser speckle velocimetry[4].

In this thesis, a new approach is taken to tackle the problem of scattering. We develop a wavefront shaping technique to steer light through opaque objects. When we shape the wavefront so that it exactly matches the scattering properties of the object, the object focuses light to a point (see Fig. 1.1b). The term 'opaque lens' was intro-
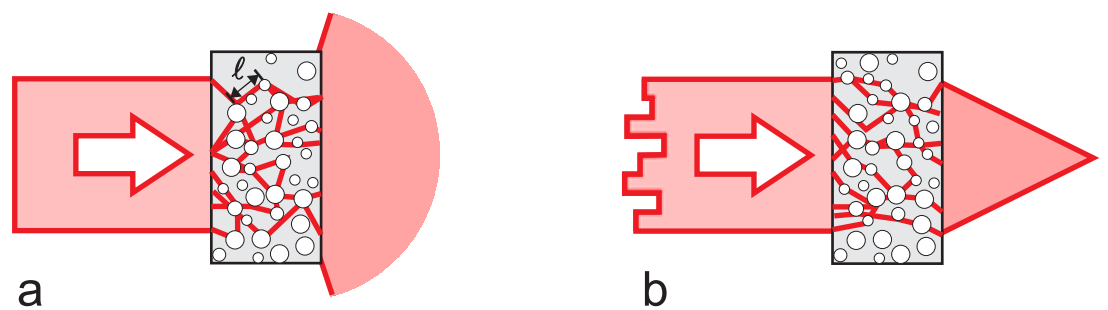

Figure 1.1: Principle of opaque lensing. a) A plane wave impinges on an opaque scattering object. In the object, light performs a random walk with a typical distance given by the mean free path for light $(\ell)$. The little light that makes it trough is scattered in all directions. b) The incident wave is shaped to match the scattering in the object. The opaque object focuses the shaped wave to any desired point, thereby acting as an 'opaque lens'. 

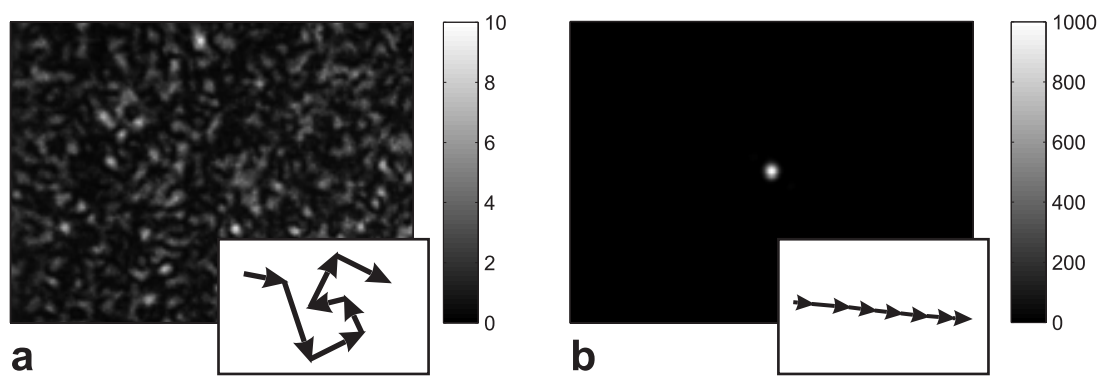

Figure 1.2: Interference in an opaque lens. a) Transmitted intensity of an unshaped incident beam. Scattered light forms a random interference pattern known as laser speckle. Inset (phasor plot), at each point many waves interfere randomly, resulting in a low overall intensity. b) Transmitted intensity of a wavefront that is optimized for focusing at a single point. The intensity in the focus is approximately 1000 times as high as the average intensity in a. Inset, in the focus all waves are in phase.

duced in a news item[5] about our research to describe this focusing behavior. Using our wavefront shaping method, we steered light through opaque objects, focused it inside and even projected simple images through the objects.

In this chapter, we first explain in general terms how we shape the wavefront to control the propagation of light. Then, we relate our work to other experimental fields. In Section 1.3, we give a brief introduction to the concepts and tools that are used for analyzing light propagation in opaque objects. We end this introductory chapter by giving an overview of this thesis.

\subsection{Opaque lenses}

The particles in a strongly scattering medium are smaller than the wavelength of light. Therefore, the wave nature of light needs to be taken into account and light propagation cannot be described in terms of light rays. Diffusion of waves is fundamentally different from diffusion of particles since waves exhibit interference. We first briefly explain how a wave propagates through a disordered medium. Then, we show how interference was used in our experiments to make an 'opaque lens' focus light.

Wave propagation in a disordered medium can be made insightful with the help of the Huygens principle[6]. When an incident beam of light hits a small particle in the object, part of the light is scattered and forms a spherical wave moving away from the particle. In turn, this spherical wave hits other particles, giving rise to more and more waves. Light propagation in a disordered scattering medium is extremely complex; light is typically scattered hundreds or thousands of times before it reaches the other side of the sample. Figure 1.2a shows the transmitted intensity for a sample that is illuminated with laser. The complicated random pattern is the result of the interference of very many different waves; this pattern is known as laser speckle.

We now illuminate the opaque object with thousands of light beams, instead of one. Each of this beams forms a different random speckle pattern when it is scattered by the object. The total field at a given point behind the sample is the sum of the 
speckle patterns of all incident beams. Since the object is disordered, each beam contributes to the field with a random phase. Therefore, the contributions from different beams mostly cancel each other (see the inset in Fig. 1.2a for a graphical representation of this statement).

Next, a spatial light modulator is used to delay each of the beams with respect to the others and thereby shape the wavefront of the incident light. The modulator is a liquid crystal on silicon display (LCoS). These displays have been developed in the last decennium for use in commercial video projectors. A computer controlled algorithm optimizes the intensity in a single target point. It does this by changing the phase of the beams one by one, until their speckle patterns are all in phase in the target. In Fig. 1.2b we show the transmission after a successful optimization. All contributions interfere constructively in the target and the intensity increases dramatically (by a factor of thousand in this case). The opaque object now sharply focuses light to a single point. The shaped wavefront uniquely matches the scattering object like a key matches a lock. When the microscopic scatterers in the object have a different position or orientation, a completely different wavefront is required. Therefore, this method only works when the scatterers do not move during the optimization.

\subsection{Relation with earlier work}

Our work is inspired by an article of Dorokhov[8] about electron scattering in a metal wire. Dorokhov predicted that there exist electron wave functions that are fully transmitted through the wire, regardless of the length of the wire. Scattering of light in a disordered medium is, in many ways, analogous to scattering of electrons in a wire. The optical equivalent of this prediction is that it should be possible to construct a shaped wave of which all $100 \%$ of the intensity is transmitted through an otherwise opaque object. We wanted to construct this wavefront. The work on opaque lenses was initially performed as a first step towards achieving full transmission. Because opaque lenses turned out to be a fascinating research subject on their own, we first explored that field for a while. Finally, we we used our optical analogue of a disordered electronic system to confirm Dorokhov's hypothesis.

In our opaque lens experiments, we 'borrowed' a lot of ideas from time-reversal experiments with ultrasound and microwaves. In pioneering work by Fink et al. (see Ref. 9 for a review) a short pulse impinged on a strongly scattering system. The amplitude of the scattered wave was recorded and played back reversed in time. Thanks to time reversal symmetry, the wave focused back to the original source. In a series of beautiful experiments, it was shown that time-reversal can be used to focus light through a disordered medium[10], break the diffraction limit[11, 12], and improve communication by using disorder[12].

In this thesis we show experiments that use our wavefront shaping method to obtain similar result with light. Our approach has two fundamental advantages over time-reversal methods. First of all, no time reversal symmetry is required. And secondly, for time reversal one needs to have a source at the target focus. With our method, it is sufficient to only have a detector in the target focus. This difference allows us to focus waves inside a scattering medium, as is demonstrated in Chapter 5. An optical method that is related to time-reversal is optical phase conjugation[13]. 
Using a non-linear crystal and high power lasers, it is possible to reverse both the direction and the phase of a speckle pattern to project the light back to its original source. Like with time-reversal, this method requires a source at the point where we want the waves to focus.

Our experimental apparatus is similar to the setups used in adaptive optics[14]. Adaptive optics is a technique for correcting aberrations in lenses or other transparent media, such as a turbulent atmosphere[15] or the human eye[16]. Adaptive optics works for situations where light propagates along rays. Diffraction and interference effects are not taken into account. In opaque scattering media, however, there are no rays of light that one can steer; light propagation is dominated by diffraction and interference. Therefore, our method uses interference instead of ray optics to steer light.

\subsection{Mathematical tools for analyzing complex system}

The propagation of light is described perfectly well by Maxwell's equations, so why would we need anything else? The problem is that these equations are so hard to solve that exact results can only be found for very simple geometries. Even for a simple sphere geometry the result is not a closed expression, but a complicated sum of Bessel functions.[17] Obviously, this approach cannot be scaled to a system containing billions of spheres, let alone irregularly shaped grains. Even worse, we do not even know the exact positions and orientations of the scatterers in a sample to begin with.

Although this seems to be a hopeless situation, it is possible to capture the overall characteristics of the system using statistical and physical tools. In this section, we introduce the most important statistical tools that are used in this thesis: ensemble averaging, correlation functions and probability density functions. Then, we highlight the powerful physical concept of diffusion. These tools are applicable to all complex systems, whether it is light propagation in a disordered medium, or the dynamics of a complex biological, chemical or economical system.

\subsubsection{Ensemble averaging}

Every sample scatters light in a unique way. Even if all macroscopic properties (layer composition and thickness, porosity, etcetera) are the same, the microscopic structure of two samples will be completely different. Therefore, it is impossible to predict the exact scattering properties of a specific sample.

Instead, one calculates average quantities for a whole ensemble of samples. For example, we could calculate the optical field averaged over all conceivable samples that consist of a $10 \mu \mathrm{m}$-thick layer of zinc oxide pigment. We write this quantity as $\langle E\rangle$, where $E$ is the field and $\langle\cdot\rangle$ denotes averaging over the ensemble of all possible samples of a given type.

In an experiment it is, most of the time, not needed to average over an ensemble of samples. Instead, one can average over the response of different portions of the sample. When both averaging methods are equivalent, the system is called ergodic. We assume ergodicity for all our samples. 


\subsubsection{Correlation functions}

The majority of the research in random scattering is concerned with calculating or measuring correlations of some kind. A correlation function relates the value of a quantity at one coordinate to the value of that quantity at a different coordinate ${ }^{1}$. For instance, the position correlation function of the electrical field is defined as

$$
C_{E}\left(\mathbf{r}_{1}, \mathbf{r}_{2}\right) \equiv\left\langle E^{*}\left(\mathbf{r}_{1}\right) E\left(\mathbf{r}_{2}\right)\right\rangle,
$$

where $^{*}$ denotes the complex conjugate. When two quantities are statistically independent, they can be averaged separately. For instance, when two points $\mathbf{r}_{1}$ and $\mathbf{r}_{2}$ are so far apart that the fields at both points are uncorrelated we can write (note the essential difference in the placement of the brackets)

$$
C_{E}\left(\mathbf{r}_{1}, \mathbf{r}_{2}\right)=\left\langle E^{*}\left(\mathbf{r}_{1}\right)\right\rangle\left\langle E\left(\mathbf{r}_{2}\right)\right\rangle \quad \text { for } \mathbf{r}_{1} \text { far from } \mathbf{r}_{2} .
$$

Since $E$ oscillates rapidly around 0 , it quickly averages out. Inside a diffusive medium $\langle E\rangle \approx 0$, and Eq. (1.2) vanishes. However, in general the decomposition in Eq. (1.2) cannot be made. Especially when $\mathbf{r}_{1}=\mathbf{r}_{2}$, the correlation function cannot be separated

$$
C_{E}(\mathbf{r}, \mathbf{r})=\left\langle E^{*}(\mathbf{r}) E(\mathbf{r})\right\rangle \neq\left\langle E^{*}(\mathbf{r})\right\rangle\langle E(\mathbf{r})\rangle
$$

We adopt the convention that the intensity $I$ (unit $\mathrm{Wm}^{-2}$ ) is defined as $I \equiv|E|^{2}$ (see e.g. [19]). Using this convention, $C_{E}(\mathbf{r}, \mathbf{r})$ equals the average intensity. Since the intensity is always positive, its average will not vanish and $C_{E}(\mathbf{r}, \mathbf{r})$ is positive.

\subsubsection{Probability density functions}

The probability density function (pdf) gives the probability that a variable has a certain value. An important pdf is that of the intensity $I$ of a speckle[20]

$$
p(I)=\frac{1}{\langle I\rangle} \exp \left(-\frac{I}{\langle I\rangle}\right),
$$

Eq. (1.4) tells us that the most likely intensity in a speckle pattern is zero. The chance of finding bright speckles decreases exponentially with intensity of that speckle. A typical speckle pattern with this distribution is shown in Fig. 1.2a.

A different pdf that is used extensively in this thesis describes the field of a speckle. The joint probability density of the real part of the field $\left(E_{r}\right)$ and the imaginary part of the field $\left(E_{i}\right)$ is given by a so-called circular Gaussian distribution

$$
p\left(E_{i}, E_{r}\right)=\frac{1}{\pi\langle I\rangle} \exp \left(-\frac{\left|E_{i}\right|^{2}+\left|E_{r}\right|^{2}}{\langle I\rangle}\right) .
$$

The Gaussian distribution is very common and always arises when many uncorrelated random variables with a finite variance are added. This important statistical fact is known as the Central Limit Theorem. In the case of a speckle pattern, the field in a single speckle is the sum of the contributions from a large number of light paths. When these light paths are independent, the field has a Gaussian distribution ${ }^{2}$.

\footnotetext{
${ }^{1}$ Here 'coordinate' can denote position, angle, frequency or any other independent variable. It is also very common to have correlation functions involving four or more coordinates.

${ }^{2}$ The assumption of independent paths is not completely true. Tiny deviations from Gaussian statistics
} 


\subsubsection{The diffusion equation}

The average propagation of intensity in a disordered medium can be described very well with a diffusion equation. For continuous wave illumination, the stationary diffusion equation[22, 23] applies

$$
-D \nabla^{2} I(\mathbf{r} ; t)=S(\mathbf{r} ; t)
$$

where $D$ is the diffusion constant for light, $S$ is the source of diffuse light, and $\nabla^{2}$ is the Laplace operator.

We now solve the diffusion equation to find the intensity distribution in a sample. All our samples are effectively infinitely large in the $x$ and $y$ directions and have a finite thickness $L$ in the $z$ direction. For such a geometry, it is convenient to work in Fourier transformed coordinates $\mathbf{q}_{\perp} \equiv\left(q_{x}, q_{y}\right)$ for the traversal coordinates $x$ and $y$. In these coordinates, Eq. (1.6) transforms to

$$
q_{\perp}^{2} I\left(\mathbf{q}_{\perp}, z\right)-\frac{\partial^{2} I\left(\mathbf{q}_{\perp}, z\right)}{\partial z^{2}}=\frac{S\left(\mathbf{q}_{\perp}, z\right)}{D},
$$

with $q_{\perp} \equiv\left|\mathbf{q}_{\perp}\right|$. We use the Dirichlet boundary conditions $I\left(-z_{e 1}\right)=0$, and $I\left(L+z_{e 2}\right)=$ 0 to describe the interfaces of the sample. These boundary conditions give a much simpler and more insightful result than the slightly more accurate mixed boundary conditions. [24] Here, $L$ is the thickness of the sample and $z_{e 1}$ and $z_{e 2}$ are the so called extrapolation lengths that account for reflection at the front and back surface of the medium, respectively. These extrapolation lengths depend on the effective refractive index of the sample and the refractive index of its surroundings[26-28]. When a slab of diffusive material is illuminated by an external source, the incident light can be described by a diffuse source at a depth of one mean free path $\ell$.[29] Then, the solution to Eq. (1.7) is (see e.g. Ref. [30])

$$
I\left(\mathbf{q}_{\perp}, z\right)=\left\{\begin{array}{ll}
S\left(\mathbf{q}_{\perp}\right) \frac{\sinh \left(q_{\perp}\left[L_{e}-z-z_{e 1}\right]\right) \sinh \left(q_{\perp}\left[\ell+z_{e 1}\right]\right)}{D q_{\perp} \sinh \left(q_{\perp} L_{e}\right)} & z \geq z_{0} \\
S\left(\mathbf{q}_{\perp}\right) \frac{\sinh \left(q_{\perp}\left[L_{e}-\ell-z_{e 1}\right]\right) \sinh \left(q_{\perp}\left[z+z_{e 1}\right]\right)}{D q_{\perp} \sinh \left(q_{\perp} L_{e}\right)} & z<z_{0}
\end{array},\right.
$$

with $L_{e} \equiv L+z_{e 1}+z_{e 2}$. A numerical Fourier transform gives $I$ in real space coordinates. The intensity distribution in a diffusive slab is shown in Fig. 1.3. The intensity is maximal close to the source and spreads out over the medium. Far away from the source, the intensity decays exponentially with distance. From an expansion of Eq. (1.8) in small variable $q_{\perp}$, we find that the intensity decreases exponentially with a decay length of $L_{e} / \sqrt{6}$.

\subsection{Outline of this thesis}

This thesis describes experiments and theory on controlling the propagation of light in opaque objects. The theoretical framework was developed in parallel with the experiments. Therefore, most of the theory is presented together with the experimental

have been observed experimentally.[21] In Chapter 9 we describe an experiment in which we observed very large effects of correlations between paths. 
a

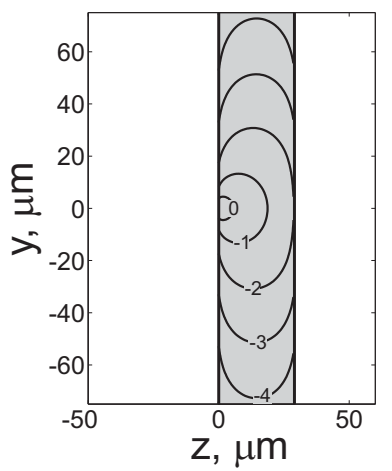

b

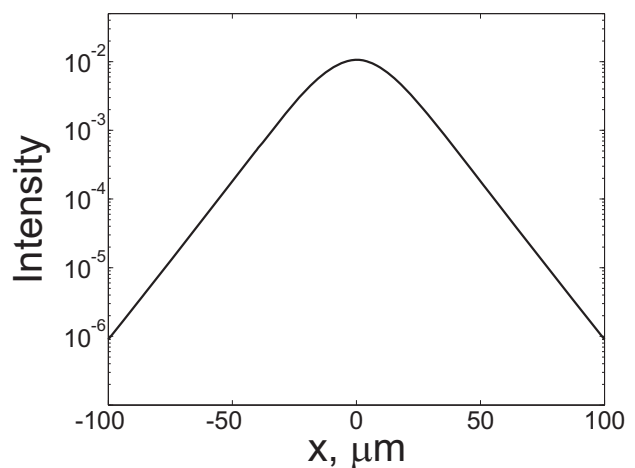

Figure 1.3: Average distribution of diffuse light in a slab (shaded rectangle of thickness $30 \mu \mathrm{m}$ ) of strongly scattering material. a) Contour plot of the energy density distribution inside the sample. The source is placed at a depth of $z=\ell=0.72 \mu \mathrm{m}$ and $x=y=0$. The numbers on the equal-density curves indicate $\log ^{10}(I)$. b) Logarithmic plot of the intensity profile at the back of the sample (at $z=30 \mu \mathrm{m}$ ).

results. The simple notation that was used in the first experiments did not suffice to describe the more advanced experiments and a more powerful notation was developed along with the theory. Therefore there are slight differences in the notation in the different chapters. Care has bee taken that all chapters are self-contained and can be read and understood separately.

In Chapter 2 we describe the experimental apparatus, the samples and the control program that were used in the experiments. Special attention is given to a novel wavefront modulation method that we developed.

In Chapter 3 the first experimental results of opaque lensing are presented. We also explain the optimization algorithm and calculate the maximally achievable intensity of the focus. The focusing resolution of opaque lenses is studied quantitatively in Chapter 4. It appears that an opaque lens focuses light as sharply as the best possible lens. In Chapter 5 the concept of opaque lenses is extended to focus light inside an opaque object. In Chapter 6 we demonstrate that disorder can improve optical communication. In Chapter 7 dynamic algorithms for use with non-stationary samples are investigated.

In Chapter 8 a transport theory for optimized wavefronts is developed. We show that wavefront shaping significantly increases the total transmission through opaque objects. The experimental observation of this effect is presented in Chapter 9.

Finally, in Chapter 10, we summarize our findings and give an outlook of the many possible applications of our work.

\section{Bibliography}

[1] Waves and imaging through complex media, edited by P. Sebbah (Kluwer Academic, Dordrecht, Netherlands, 2001).

[2] D. Huang, E. A. Swanson, C. P. Lin, J. S. Schuman, W. G. Stinson, W. Chang, M. R. Hee, T. 
Flotte, K. Gregory, and C. A. Puliafito, Optical coherence tomography, Science 254, 1178 (1991).

[3] A. Yodh and B. Chance, Spectrocopy and imaging with diffusing light, Phys. Today 48, 34 (1995).

[4] J. D. Briers, Laser Doppler, speckle and related techniques for blood perfusion mapping and imaging, Physiol. Meas. 22, R35 (2001).

[5] P. Schewe, Opaque lens, Physics News Update 835, 1 (2007).

[6] C. H. R. Huygens, Traité de la lumière (van der Aa, Leiden, 1690), as discussed in Ref. [7].

[7] A. E. Shapiro, Huygens' 'Traité de la lumière' and Newton's 'Opticks': Pursuing and eschewing hypotheses, Notes and Records Roy. Soc. Lond. 42, 223 (1989).

[8] O. N. Dorokhov, Coexistence of localized and extended electronic states in the metallic phase, Sol. St. Commun. 51, 381 (1984).

[9] M. Fink, D. Cassereau, A. Derode, C. Prada, P. Roux, M. Tanter, J.-L. Thomas, and F. Wu, Time-reversed acoustics, Rep. Prog. Phys. 63, 1933 (1999).

[10] M. Fink, C. Prada, F. Wu, and D. Cassereau, Self focusing in inhomogeneous media with time reversal acoustic mirrors, IEEE Ultrason. Symp. Proc. 2, 681 (1989).

[11] A. Derode, P. Roux, and M. Fink, Robust acoustic time reversal with high-order multiple scattering, Phys. Rev. Lett. 75, 4206 (1995).

[12] G. Lerosey, J. de Rosny, A. Tourin, and M. Fink, Focusing beyond the diffraction limit with far-field time reversal, Science 315, 1120 (2007).

[13] Optical phase conjugation, edited by R. A. Fisher (Academic Press, New York, 1983).

[14] R. K. Tyson, Principles of adaptive optics, 2nd ed. (Academic Press, New York, 1998).

[15] Adaptive optics in astronomy, edited by F. Roddier (Cambridge University Press, Cambridge, 1997).

[16] Special issue: Advances in retinal imaging, J. Opt. Soc. Am. A 24, 1223 (2007).

[17] G. Mie, Beiträge zur Optik trüber Medien, speziell kolloidaler Metallösungen, Ann. Phys. 330, 377 (1908), as discussed in Ref. [18].

[18] H. C. van de Hulst, Light scattering by small particles, 1981 ed. (Dover Publications, Inc., New York, 1957).

[19] M. C. W. van Rossum and T. M. Nieuwenhuizen, Multiple scattering of classical waves, Rev. Mod. Phys. 71, 313 (1999).

[20] J. W. Goodman, Statistical optics (Wiley, New York, 2000).

[21] J. F. de Boer, M. C. W. van Rossum, M. P. van Albada, T. M. Nieuwenhuizen, and A. Lagendijk, Probability distribution of multiple scattered light measured in total transmission, Phys. Rev. Lett. 73, 2567 (1994).

[22] H. S. Carslaw and J. C. Jaeger, Conduction of heat in solids, 2 ed. (University Press, 1959).

[23] S. Chandrasekhar, Radiative transfer (Dover Publications, Inc., New York, 1960).

[24] see e.g. Ref. 25 for an exact solution of Eq. (1.7) using mixed boundary conditions.

[25] I. M. Vellekoop, P. Lodahl, and A. Lagendijk, Determination of the diffusion constant using phase-sensitive measurements, Phys. Rev. E 71, 056604 (2005).

[26] A. Lagendijk, R. Vreeker, and P. de Vries, Influence of internal reflection on diffusive transport in strongly scattering media, Phys. Lett. A 136, 81 (1989).

[27] J. X. Zhu, D. J. Pine, and D. A. Weitz, Internal reflection of diffusive light in random media, Phys. Rev. A 44, 3948 (1991).

[28] M. U. Vera and D. J. Durian, Angular distribution of diffusely transmitted light, Phys. Rev. E 53, 3215 (1996).

[29] E. Akkermans, P. E. Wolf, and R. Maynard, Coherent backscattering of light in disordered media: Analysis of the peak line shape, Phys. Rev. Lett. 56, 1471 (1986).

[30] K. L. van der Molen, Experiments on scattering lasers from Mie to random, Ph.D. thesis, Univerity of Twente, Enschede, 2007, available on http://cops.tnw.utwente.nl. 


\section{Chapter 2}

\section{Experimental apparatus}

In this chapter we discuss the experimental apparatus that we built to control the propagation of light in disordered media. We discuss the considerations that played a role in designing the experiment and give special attention to a new wavefront shaping method that we developed for our experiments. The setup was modified for each of the individual experiments that are described in this thesis. Here we discuss the common elements of the design and explain what specific modifications were made.

A general diagram of the experiments is shown in Fig. 2.1. A strongly scattering sample is illuminated by a wavefront synthesizer that is able to construct a spatially modulated beam. The light that is scattered by the sample is collected by a detection system. This detection system provides feedback to a computer algorithm that controls the wavefront synthesizer.

The most complex part of the apparatus is the wavefront synthesizer. It contains a liquid crystal display (LCD) that spatially modulates incident light. In Section 2.1 we analyze the characteristics of the LCD and explain how it was used as a phase modulator. We also developed a new method for using the LCD to spatially modulate both amplitude and phase of the light. This method was published in Ref. 1. In Section 2.2 the detection system is described. Here we also discuss how the detection is synchronized with the wavefront synthesizer. We comment on the overall stability issues of the setup in Section 2.3. Then, in Section 2.4, we describe which types of samples were used and introduce a new fabrication method that we developed for making strongly scattering samples. The structure of the control program that coordinates the experiment is discussed in Section 2.5. Finally, in Section 2.6 we give an outlook of what can be expected in future experiments.

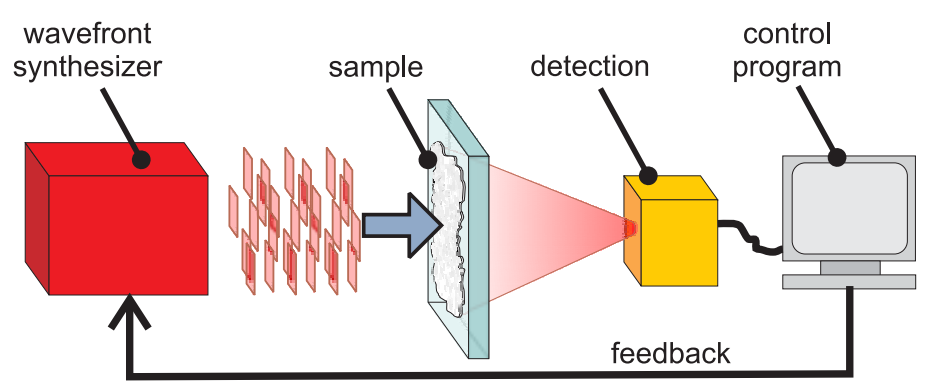

Figure 2.1: Block scheme of the experiment. A wavefront synthesizer generates a shaped monochromatic wavefront. The light is scattered by a strongly scattering sample. A detector defines a target position for the optimization procedure and provides a feedback signal. A computer analyzes the signal and reprograms the phase modulator until the light optimally focuses on the target. 

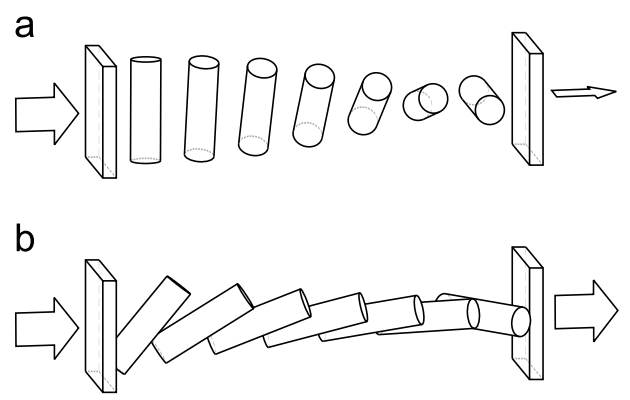

Figure 2.2: Operating principle of a transmissive TN LCD. a) No voltage is applied and the rod-like liquid crystal molecules are ordered in a helix. The polarization of incident light follows the twist. b) A voltage is applied between the two electrodes. The rods orient in the direction of the field and the twist disappears. The polarization of the incident light is not rotated.

\subsection{Wavefront synthesizer}

Computer controlled wavefront shaping is a very versatile technique that is used in many fields of optics. In adaptive optics[2], for example, deformable mirrors or other spatial light modulators are used to correct aberrations in a variety of optical systems. Another area that relies on computer controlled light modulators is that of digital holography. Topics in digital holography include holographic data storage[3], 3D display technology[4], and holographic image processing[5].

Liquid crystal displays are among the most popular types of light modulators because of their high optical efficiency, the high number of degrees of freedom and their wide availability. In our experiment, light is modulated with a twisted nematic (TN) liquid crystal display ${ }^{1}$. This LCD can modulate light at a refresh rate of 60 or $72 \mathrm{~Hz}$ at a resolution of $1024 \times 768$ pixels. In Section 2.1 .1 we discuss the operating principle of a TN LCD. LCDs are designed to modulate light intensity. To use the LCD as a phase modulator, a thorough characterization of the display is required. This characterization procedure is described in Section 2.1.2. In Section 2.1.3, we describe a common method to use TN LCDs as phase modulators. This method was used to perform the experiments that are described in Chapters 3 and 7 . For the other experiments in this thesis, we developed a new modulation method that has several advantages over existing methods. This novel modulation technique is introduced in Section 2.1.4 and experimentally demonstrated in Section 2.1.5. The switching behavior of the display is described in Section 2.1.6.

\subsubsection{Principle of a twisted nematic liquid crystal display}

The operating principle of LCDs is based on the birefringence of rod-like organic molecules. In the twisted nematic phase, these rods are ordered in a helix as is shown in Fig 2.2a. A transmissive LCD is typically sandwiched between two crossed polar-

\footnotetext{
${ }^{1}$ We used two Holoeye LC-R 2500 systems. The LCDs have a size of $19.6 \mathrm{~mm} \times 14.6 \mathrm{~mm}$. One system was customized by the manufacturer to control two LCDs with one control box. That system was used for the experiments in Chapter 9, where we needed control over both polarizations.
} 
izers. The $90^{\circ}$ helical structure of the liquid crystal rotates the angle of polarization of the incident light so that the light passes the second polarizer. When a voltage is applied over the liquid crystal cell, the molecules align with the electrical field, as is shown in Fig 2.2b. Now the optical axis of the molecules is parallel to the direction of light propagation and the polarization of the incident light is not rotated. As a result, the light is blocked by the second polarizer and the pixel is dark.

The LCDs in our experiments are TN liquid crystal on silicon (LCoS) displays. These reflective displays are designed to be used with a polarizing beam splitter or with crossed polarizers in an off axis configuration. The thickness of the liquid crystal layer and the twist angle are carefully engineered to optimize brightness, contrast and response time for projecting video.

The operation principle of a reflective LCD is more complicated than that of transmissive LCD (see e.g. Ref. 6). When no voltage is applied, the polarization of the linearly polarized incident light follows the helix. At the back surface of the LCD the light is reflected and, on its way back it is rotated back to its original polarization. When a voltage is applied, the helix is distorted (see Fig. 2.2b). Now, linearly polarized light cannot completely follow the twist anymore and becomes elliptically polarized. In the 'on' state, the light is exactly circularly polarized at the back surface of the LCD. Reflecting circularly polarized light changes its handedness. Therefore, on its way back, the polarization is not rotated back to its original polarization, but to the orthogonal polarization state. In the crossed polarizer configuration, the pixel now is reflecting.

\subsubsection{Liquid crystal display characterization}

The optical characteristics of a single pixel of the LCD can be described by its Jones matrix $J$. The Jones matrix relates the horizontal and vertical polarization components of the incident field (denoted as, respectively $E_{H}^{\text {in }}$ and $E_{V}^{\text {in }}$ ) to the components of the outgoing field

$$
\left[\begin{array}{c}
E_{H}^{\text {out }} \\
E_{V}^{\text {out }}
\end{array}\right]=J\left[\begin{array}{c}
E_{H}^{\text {in }} \\
E_{V}^{\text {in }}
\end{array}\right] .
$$

Elliptic and circular polarization are described by complex values of $E_{H}$ and $E_{V}$. In general, the elements of the Jones matrix are also complex numbers. A pixel of the LCD is fully characterized by measuring $J(V)$ for all voltage settings $V$.

The setup in Fig. 2.3a was used to measure the Jones matrix of the LCD. A laser illuminates a disk with a diameter of approximately $3 \mathrm{~mm}$ in the center of the LCD. With two waveplates, any polarization of incident light can be generated. A third waveplate and a polarizer are used to analyze the modulated light in any desired polarization basis. The modulated light is focused on a detector. To obtain, for instance, the $J_{12}$ component, the polarization optics were rotated so that the incident light was vertically polarized and the reflected horizontally polarized light was analyzed. We later used the simpler setup that is shown in Fig. 2.3b. That setup has no polarization optics that need to be rotated and, therefore, is less sensitive to alignment inaccuracies. With the simplified setup only the $J_{12}$ component can be measured. For the 


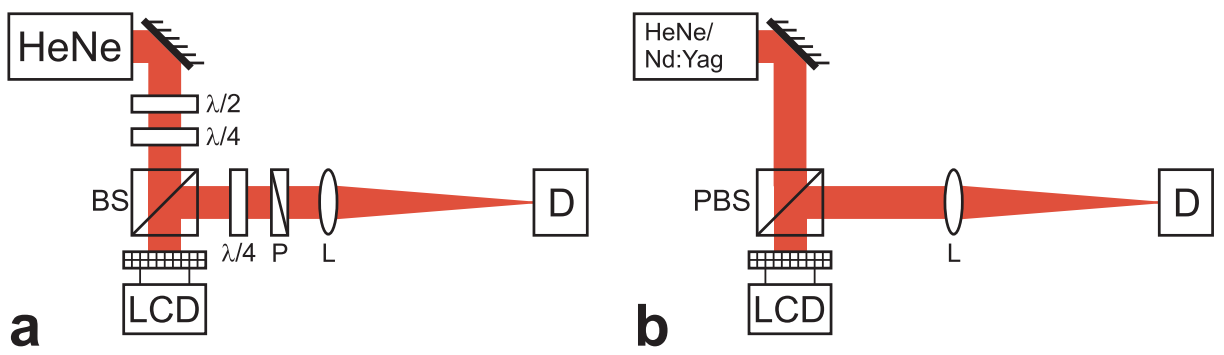

Figure 2.3: Setups used for characterizing the LCD. a) Setup for full characterization. $\lambda / 2$, half waveplate; $\lambda / 4$, quarter waveplate; BS, non-polarizing $50 \%$ beam splitter; $P$, polarizer; $L, 60 \mathrm{~cm}$ lens; D, detector. The light source was a $632.8 \mathrm{~nm}$ HeNe laser. b) Setup for partial characterization. PBS, polarizing beam splitter. Partial characterization was performed at wavelengths of $632.8 \mathrm{~nm}$ (HeNe laser) and $532 \mathrm{~nm}$ ( $\mathrm{Nb}$ :YAG laser).

modulation scheme that is discussed in Section 2.1.4, such a partial characterization of the LCD is sufficient.

In both setups, $J_{12}$ was measured using a diffractive technique that is comparable to the method described in Refs. 7 and 8 but only required detection of the $0^{\text {th }}$ order diffraction mode. We assumed that all pixels of the LCD have the same Jones matrix. First the absolute value of $J_{12}(V)$ was obtained by varying the voltage over all pixels of the LCD simultaneously. Then, we programmed the LCD with a binary grating with a duty cycle of $50 \%$ (a so called Ronchi grating). The voltage over the notches of the grating was kept constant at $V_{0}$ while the voltage over the rules was varied. The intensity in the $0^{\text {th }}$ diffraction order responds as

$$
\frac{I\left(V, V_{0}\right)}{\left|E_{H}^{\text {in }}\right|^{2}}=\left|J_{12}\left(V_{0}\right)\right|^{2}+\left|J_{12}(V)\right|^{2}+2\left|J_{12}\left(V_{0}\right)\right|\left|J_{12}(V)\right| \cos \left[\arg J_{12}(V)-\arg J_{12}\left(V_{0}\right)\right],
$$

which gives us, in principle, enough information to obtain the phase of $J_{12}(V)$ up to an overall phase offset. In practice, however, the inversion of Eq. (2.2) is very sensitive to noise for some values of $V$. We solved this problem by measuring $I\left(V, V_{0}\right)$ for different values of $V_{0}(0 \%, 25 \%, 50 \%, 75 \%$ and $100 \%$ of the maximum voltage). Then we combined the data, using only measurements for which the inversion of Eq. (2.2) was stable. The result of these measurements is shown in Fig. 2.4. We find that the phase of the reflected light changes from $\pi$ to 0 with increasing voltage. The amplitude increases from 0 to a maximum at the 'on' state (around a phase of $35^{\circ}$ ) and then decreases slightly.

To obtain all four elements of the Jones matrix, the measurement was repeated for each element of $J$. Moreover, the measurements were also performed in a rotated basis with left and right hand circularly polarized light. These extra measurements resolved the relative phase of the different components of the $J$ matrix.

All in all, measuring the full Jones matrix of an LCD is cumbersome. A complicating factor is the fact that all polarization states (except for horizontal or vertical polarization) are changed by reflecting off a coated mirror or passing through a beam splitter. Theoretical analysis is complicated by the fact that in thin LCDs surface effects start 


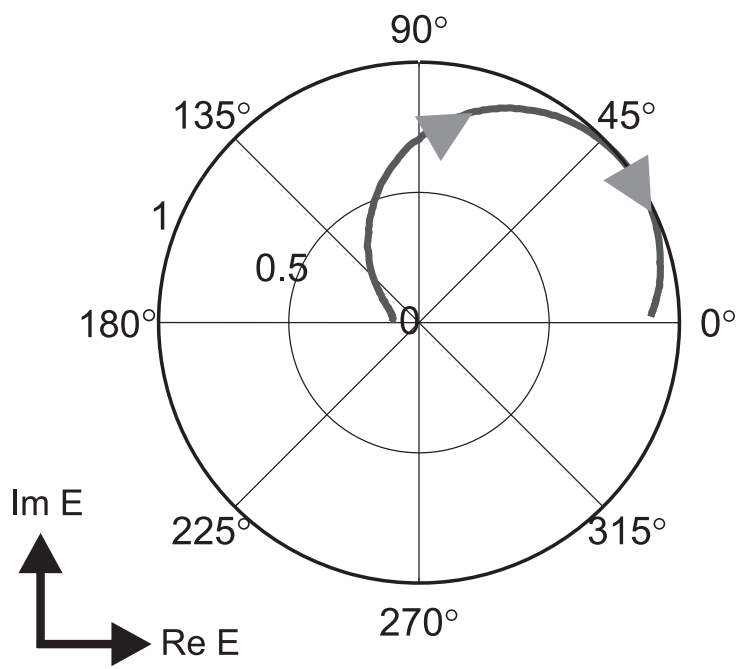

Figure 2.4: Polar plot of the modulator output amplitude with vertical polarization in, horizontal polarization out $\left(J_{12}\right)$, at a wavelength of $532 \mathrm{~nm}$. The modulation voltage increases in the direction of the gray arrows.

to play a role. Also, the total amount of light that was reflected by the LCD was found to depend on the voltage, which means that the Jones matrix is not unitary. Therefore, many of the theoretical models that are used to describe LCDs[9-11] can only give an approximate result.

\subsubsection{Liquid crystal phase-mostly modulation}

TN LCoS displays for intensity modulation typically achieve a maximum phase retardation of around $\pi$ for horizontally or vertically polarized light. Nevertheless, by choosing a suitable combination of incident polarization and analyzer orientation, it is often possible to find an operating mode where the phase retardation is $2 \pi$ while the amplitude modulation is relatively low. This mode of operation is called 'phasemostly' modulation and has been the subject of extensive experimental and theoretical research[7, 8, 10-18].

The optimal configuration of the polarizers is different for each specific series of LCDs and also depends on the wavelength of the light. In general, the optimal combination of polarization states is elliptical.[17] We found the optimal polarizations for a wavelength of $632.8 \mathrm{~nm}$ with a brute force optimization method. This method used the measured Jones matrix of the LCD to numerically try all configurations. In the optimal configuration the transmission is low and the system is very sensitive to variations in the angles of the waveplates. The measured modulation curve (see Fig. 2.5) has an intensity variation of $21 \%$ of the mean value. This configuration was used successfully in the wavefront shaping experiments that are described in Chapters 3 and 7. In these experiments, a reference detector compensated for the intensity variations. 


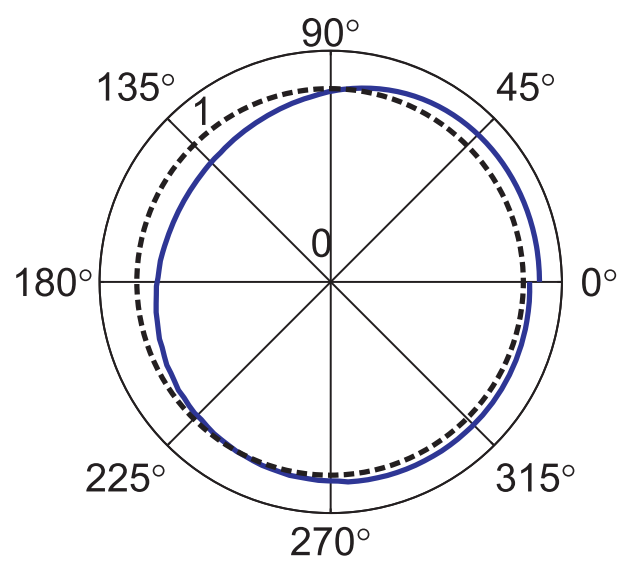

Figure 2.5: Polar plot of the modulator output amplitude. Dashed circle, perfect phase only modulation; solid curve, measured response in the configuration for optimal phase-mostly modulation at a wavelength of $632.8 \mathrm{~nm}$.

\subsubsection{Decoupled amplitude and phase modulation ${ }^{2}$}

In this section, we describe a novel modulation scheme that uses a TN LCD in combination with a spatial filter to achieve individual control over the phase and the amplitude of the light. The method has four major advantages over the scheme described in the previous section. Firstly, it allows separate control over amplitude and phase of the wavefront. Secondly, the residual amplitude-phase cross-modulation that occurred with the phase-mostly modulation scheme is almost completely eliminated. Thirdly, the LCD operates in a simple horizonal-in vertical-out configuration, this saves components and characterization time, and makes the setup less sensitive to align. And finally, the experimental setup can be easily extended to control both polarizations.

Since the introduction of liquid crystals, many techniques have been developed to achieve combined amplitude and phase modulation. Examples are setups where two LCDs are used to compensate amplitude-phase cross modulation[19, 20], and double pixel setups where two pixels are combined to a macropixel[21-23]. Each of this techniques has its own limitations. The use of two LCDs introduces alignment and synchronization issues. Dual pixel schemes put specific demands on the modulation capacities of the LCD, such as requiring phase-only modulation[23], amplitude-only modulation[21] or a phase modulation range of $2 \pi$ [22].

We developed a method that combines four pixels into a macropixel. With this method, full spatial amplitude and phase modulation can be achieved with any LCD. The setup used for this modulation scheme is shown in Fig. 2.6. A monochromatic beam of light is incident normal to the SLM surface. The modulated light is reflected from the SLM. We choose an observation plane at which the contribution of each

${ }^{2}$ This section and the following section are based on the article Spatial amplitude and phase modulation using commercial twisted nematic LCDs, E. G. van Putten, I. M. Vellekoop, and A. P. Mosk, accepted for publication in Applied Optics (2008). 


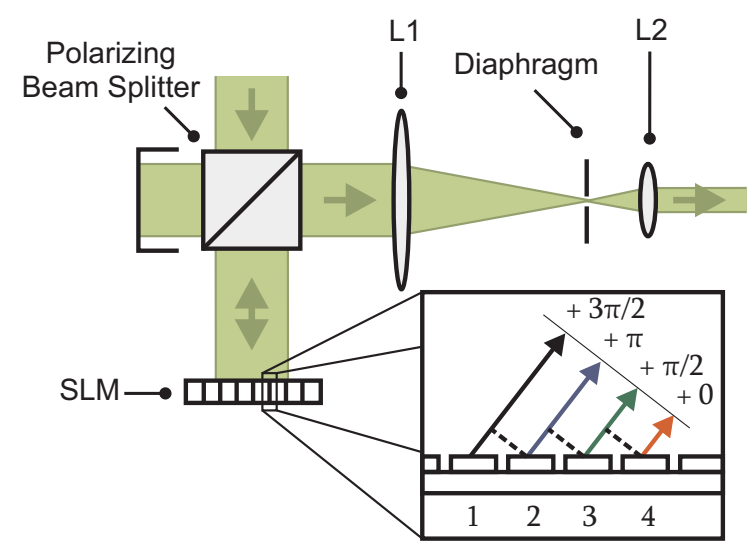

Figure 2.6: Experimental setup to decouple phase and amplitude modulation. Four neighboring pixels form one macropixel which can modulate amplitude and phase. In the plane of observation neighboring pixels are $\pi / 2$ out of phase (inset). A spatial filter combines the pixels into one macropixel. $L_{1}, L_{2}$, lenses with a focal distance of $250 \mathrm{~mm}$ and $200 \mathrm{~mm}$, respectively.

neighboring pixel is $\pi / 2$ out of phase, as is seen in the inset. A spatial filter removes all higher harmonics from the generated field, so that four neighboring pixels are merged into one macropixel.

By choosing the correct combination of pixel values, any complex value of the total field can be synthesized. The electric field in a macropixel, $E_{\mathrm{sp}}$, can be written as the sum of the fields, $E_{1}, E_{2}, E_{3}$, and $E_{4}$, coming from the four different pixels. Behind the spatial filter, $E_{\mathrm{sp}}$ is given by

$$
\begin{aligned}
E_{\mathrm{sp}} & =E_{1} \exp \left(\frac{3}{2} i \pi\right)+E_{2} \exp (i \pi)+E_{3} \exp \left(\frac{1}{2} i \pi\right)+E_{4}, \\
& =\left(E_{4 r}-E_{2 r}\right)+i\left(E_{3 r}-E_{1 r}\right)+\left(E_{1 i}-E_{3 i}\right)+i\left(E_{4 i}-E_{2 i}\right),
\end{aligned}
$$

where the indices $r$ and $i$ refer to the real and the imaginary part of the field. The voltages on pixels 2 and 4 are chosen such that $E_{4 i}-E_{2 i}=0$, and in the same way the voltages on pixels 1 and 3 are chosen such that $E_{1 i}-E_{3 i}=0$. Equation 1 is now reduced to

$$
E_{\mathrm{sp}}=\left(E_{4 r}-E_{2 r}\right)+i\left(E_{3 r}-E_{1 r}\right)
$$

The separate pixels are programmed so that the fields are given by

$$
\begin{aligned}
& E_{1}=-A \sin (i \phi)+i \Delta_{1}, \\
& E_{2}=-A \cos (i \phi)+i \Delta_{2}, \\
& E_{3}=A \sin (i \phi)+i \Delta_{1}, \\
& E_{4}=A \cos (i \phi)+i \Delta_{2},
\end{aligned}
$$

with $A$ and $\phi$, respectively, the desired amplitude and phase. The imaginary parts $\Delta$ cancel. The desired complex value is now synthesized by the real values of the field at the four different pixels. From the geometrical construction shown in Fig. 2.7 it can 


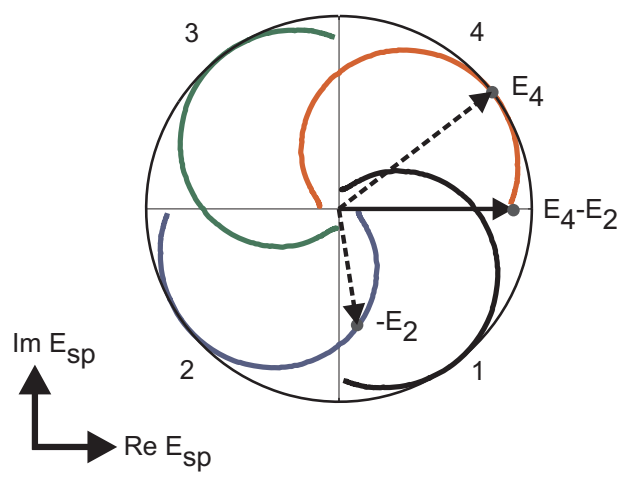

Figure 2.7: Modulation amplitude response of four pixels that form a macropixel. Pixels have a $\pi / 2$ phase shift with respect to each other. The four pixels synthesize any complex value: $E_{2}$ and $E_{4}$ generate the real part of the field; $\operatorname{Im}\left(E_{4}-E_{2}\right)=0 . E_{1}$ and $E_{3}$ form the imaginary part (not shown).

be seen how we modulate a value on the real axis, $E_{4 r}-E_{2 r}$, by choosing $E_{4 i}=E_{2 i}$. It is always possible to find pixels values with exactly opposite imaginary parts and different real parts. The only requirement posed on the SLM is that at least one of the field components has to vary when the pixel voltages are changed.

The decoupled amplitude and phase modulation was used with a $\lambda=532 \mathrm{~nm}$ diode pumped solid state laser $^{3}$ for the experiments in Chapter 5. For the experiments in Chapters 4, 6 and 9, a $\lambda=632.8 \mathrm{~nm}$ helium neon laser was used ${ }^{4}$. In Chapter 9 the wavefront synthesizer was extended to modulate two polarizations by simply replacing the beam dump (see Fig. 2.6) by a second LCD.

\subsubsection{Demonstration of amplitude and phase modulation}

We tested the new modulation technique with the same characterization method as was used to measure the modulation curve of the LCD (see Section 2.1.2), with the difference that we now use macropixels instead of actual pixels. We programmed the modulator so that the macropixels formed a Ronchi grating. The notches were set at half of the maximum amplitude with a phase offset of zero. The phase and amplitude of the rules was varied. A detector recorded the light intensity in the $0^{\text {th }}$ diffraction order $^{5}$. These experiments were performed at a wavelength of $532 \mathrm{~nm}$.

We observed that the intensity in the $0^{\text {th }}$ diffraction order varied as the cosine of the phase difference between the notches and the rules of the grating, just as is expected from Eq. (2.2). We repeated the experiment with different amplitudes in the rules of the grating. All experimental result overlaps almost perfectly with the theoretical curves (see Fig. 2.8). From this agreement, we conclude that a full $2 \pi$ phase shift is

\footnotetext{
${ }^{3}$ Coherent Compass M315-100, max. $100 \mathrm{~mW}$, intra cavity doubled, diode pumped Nb:YAG laser.

${ }^{4}$ JDS Uniphase 1125/P, 5mW polarized HeNe laser.

${ }^{5}$ The $0^{\text {th }}$ diffraction order of the macropixel grating is the direction of the modulated light when all macropixels are set to the same amplitude and phase. This is not the same as the $0^{\text {th }}$ diffraction order of the modulator, which is the direction of the light when all actual pixels are set to the same voltage.
} 


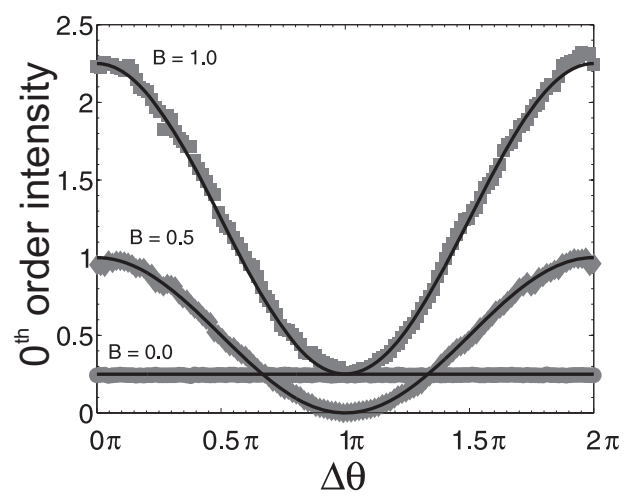

Figure 2.8: Intensity in $0^{\text {th }}$ order grating mode as a function of the set phase difference $\Delta \theta \equiv \theta_{B}-\theta_{A}$. Notches of the grating are kept at amplitude $A=0.5$, phase $\theta_{A}=0$. Amplitude $B$ and phase $\theta_{B}$ of the rulers is varied. Solid curves, expected intensity for perfect modulation. Intensities are referenced to $I_{0}=4.56 \cdot 10^{3}$ counts/second.

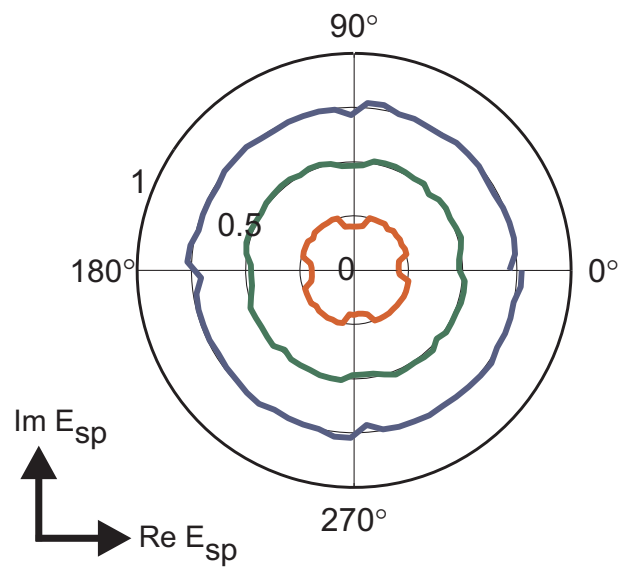

Figure 2.9: Independent phase and amplitude modulation. Curves show the measured relative amplitude $A / \sqrt{I_{0}}$ as a function of the programmed phase. Relative amplitudes are set to 0.25 , 0.5 , and 0.75 . $I_{0}=19.7 \cdot 10^{3}$ counts $/$ second.

achieved. Moreover, at $\pi$ phase shift the intensity in the $0^{\text {th }}$ order vanishes, which indicates that the field of the notches has the same magnitude and opposite sign as the field of the rules of the grating.

To determine the amount of amplitude-phase cross-modulation quantitatively, we measured the intensity in the $0^{\text {th }}$ diffraction order when all macropixels are set to the same field and amplitude. When the phase is cycled from 0 to $2 \pi$, the observed intensity remains virtually equal. From the results shown in Fig. 2.9, we find that the amplitude is constant to within $2.5 \%$, which is a significant improvement over the $21 \%$ cross-modulation observed with the phase-mostly modulation scheme. The small amplitude variations are periodic with a $\pi / 2$ period. This periodicity is understand- 
able, since after $\pi / 2$ rotation of the phase, the same pattern is programmed on the LCD shifted by one pixel (see Eqs.(2.6) - (2.9)). Unlike the phase-mostly modulation method, 0 and $2 \pi$ are equivalent, which means that there are no discontinuities at phase wraps. The amplitude variations are probably the result of dynamic cross-talk between neighboring pixels. This effect is discussed in the following section.

Although the amplitude of a macropixel is not affected by its phase, neighboring macropixels do effect each other. A detailed analysis of this effect is found in Ref. 24. The effect is similar to ordinary diffraction: the beams originating from the two macropixels expand through diffraction and interfere with each other. It was found that, typically, a completely random wavefront carries approximately $85 \%$ of the intensity of a plane wave. The rest of the intensity is clipped by the iris diaphragm. In Chapter 5 this effect was compensated for by measuring a reference intensity with a random wavefront. In Chapter 9 we used a more accurate method where the sample is translated.

\subsubsection{Transient behavior}

We now investigate how fast a pixel of the LCD can be switched. The image on the modulator is updated with a refresh rate of 60 frames per second. To allow for real time operation, we reconfigured the gamma lookup table of the LCD driver electronics. The table was configured so that a pixel value linearly corresponds to an amplitude modulation on the real or imaginary axis. Furthermore, the conversion from a polar representation (amplitude and phase) to the real and imaginary parts (see Eqs. (2.6)-(2.9)) is performed in real time by the video acceleration hardware. In the process of displaying a new image the following sequence of events can be identified:

1. The control program loads new matrices for the amplitude and phase to the video hardware

2. The video hardware scales and translates the matrices to screen coordinates. Then it performs the necessary calculations to convert amplitude and phase to pixel values and prepares the new image in a background buffer.

3. The control program waits to just before the start of a new frame (the so called vertical retrace period) to swap the background buffer with the foreground buffer.

4. After every vertical retrace the video hardware sends the image to the light modulator over a digital visual interface (DVI) link.

5. The light modulator hardware receives the image and converts pixel values to voltages with the use of an internal gamma lookup table

6. The light modulator hardware drives a matrix of transistors on the back of the LCoS display according to a pulse width modulation (PWM) scheme.

To measure the transient behavior of the LCD, we repeatedly switched the whole display from the minimum to the maximum voltage and back, each time waiting $500 \mathrm{~ms}$ between switches. A camera was configured to measure the intensity in the center 


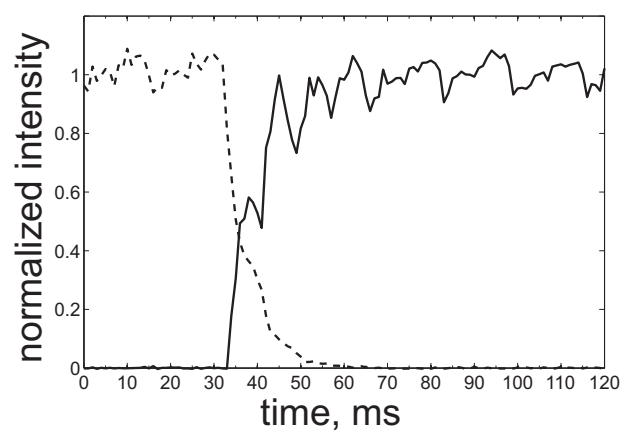

Figure 2.10: Transient intensity response of the LCD for switching the amplitude from 0 to maximum (solid curve) and for switching it back to 0 (dashed curve). During the first $33 \mathrm{~ms}$ ( 2 frames) the display does not respond at all. The intensity is normalized to the average intensity in the 'on' state.

of the modulated beam with a shutter time of $1 \mathrm{~ms}$. The delay between the vertical retrace and the camera trigger was varied from $0 \mathrm{~ms}$ to $120 \mathrm{~ms}$.

Figure 2.10 shows the measured switching response of the LCD. We recognize three distinct periods. During the first two frames (from 0 to $33 \mathrm{~ms}$ ) the image on the LCD does not change. We call this period the idle time $T_{\text {idle }}$. During the idle time the image is transferred from the computer to the modulator. In the second period the voltage over the pixels changes and the liquid crystal molecules reorient, which takes a certain time $T_{\text {settle }} \approx 50 \mathrm{~ms}$. In the third period, the liquid crystal molecules have reoriented completely. However, the signal still oscillates with a period of exactly half a frame $(8.3 \mathrm{~ms})$. These oscillations are the result of how the LCD hardware drives the pixels (see e.g. [25]). Each pixel is switched on and off according to a PWM code. A storage capacitor at each pixel integrates the total current to achieve the desired average voltage over the liquid crystal. After half a frame, the controller reverses the voltage to avoid a DC current that would damage the liquid crystal. This switching scheme results in rapid oscillations in the reflected light.

When two neighboring pixels have a different voltage, there is a field gradient at their border. Due to the pulse modulation scheme, the gradient will oscillate. The magnitude of this undesired effect depends on the PWM code of each of the pixels. For example, consider the field response for a varying phase and a constant amplitude of 0.25 (the smallest circle in Fig. 2.9). The response curve shows small jumps in the amplitude at phase values of $-15^{\circ}$ and $+15^{\circ}$, these jumps are repeated every $90^{\circ}$.

To understand these jumps, we examine the bit code of adjacent pixels in a macropixel. For a phase of $14^{\circ}$, pixel 1 and 2 have a value of 255 and 230 . At a phase of $15^{\circ}$, the first pixel values has changed to 256 and the second pixel is still at 230. Although the change from 255 to 256 appears to be small, these numbers have a completely different bit-pattern (011111111 and 100000000 respectively). Therefore, the field gradient between pixel 1 and pixel 2 will differ significantly between the two situations, resulting in a jump in the field response.

The transient switching characteristics of the LCD and the temporal oscillations put special demands on the timing of the detection. This issue is addressed in Sec- 


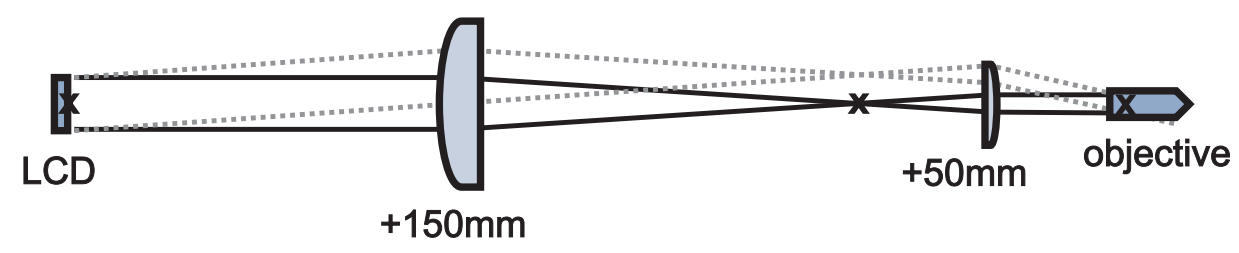

Figure 2.11: An imaging telescope is used to project the surface of the LCD to the entrance pupil of the microscope objective. Crosses indicate focal points of the lenses.

tion 2.2.

\subsubsection{Projecting the wavefront}

In most of the experiments in this thesis, the shaped wavefront is focused on the sample by means of a microscope objective. The surface of the LCD is imaged onto the entrance aperture of the microscope objective with an imaging telescope. When combined phase and amplitude modulation is used (see Section 2.1.4) there is a pinhole in the focus of the telescope to spatially filter the generated wavefront. The telescope also serves to demagnify the beam coming from the LCD to the size of the microscope objective's aperture. It is essential that a telescope with two positive lenses is used so that beams that leave the LCD at an angle are also imaged onto the aperture of the objective (see Fig. 2.11). When the lenses are aligned properly, the entrance pupil of the objective corresponds to a circular area on the LCD. Pixels outside this area do not contribute to the field on the sample and can, therefore, be skipped in the optimization procedure.

In this configuration, a pixel on the LCD corresponds to an angle in the focal plane of the objective. When we group pixels on the LCD together in blocks, we reduce the angular resolution at the sample surface and, thereby, reduce the size of the projected spot. The aperture of the objective is always filled completely. Therefore, when a high NA objective is used, the number of segments in the incident wavefront is approximately equal to the number of mesoscopic channels on the sample surface. If, for example, all pixels are grouped into a single segment, light is focused to a diffraction limited spot encompassing exactly one mesoscopic channel.

If the sample is not positioned in the focal plane of the objective, or when a low NA objective is used, the wavefront synthesizer illuminates a spot that supports more scattering channels than there are control segments in the incident wavefront. Thus the incident field cannot completely be defined by the wavefront synthesizer. It turns out that, in general, this limitation has little effect on how well the propagation of light is controlled (see for instance Fig. 3.4).

\subsection{Detection}

During the wavefront optimization procedure, a detector monitors the intensity in the target. Different detectors were used for this purpose. In this section we discuss the detectors that were used, as well as their relevant properties. 
The first experiments were performed with a photodiode. A photodiode has an excellent dynamic range and a fast response. The major drawback of using a photodiode is that it is very hard to select exactly a single speckle. For this reason we started using cameras. During optimization the camera image is integrated over a disk with a software defined radius and position. The diameter of the disk is chosen to be slightly smaller than the speckles that are visible on the initial camera image. Using a camera also allowed us to easily define multiple targets and to monitor the intensity in the background around the optimized speckle.

We have used three different models of cameras. The first model is the Allied Vision Technologies Dolphin F-145B. This camera is an all purpose charge coupled device (CCD) camera that is connected to the computer with a IEEE 1394 link (firewire). To increase the dynamic range of the camera, the shutter time was varied by the control program. In Chapter 9 the required dynamic range was so high that changing the shutter time was not sufficient. Instead, the computer controlled a motorized translation stage that automatically placed a neutral density filter in front of the camera.

We used the Dolphin camera for all experiments, except for the fluorescence measurements described in Chapter 5. For that experiment, we started with a Hamamatsu ORCA electron multiplying CCD (EMCCD) camera. This camera is cooled with a Peltier element to reduce the dark current. Also, the signal is amplified on the CCD chip to overcome readout noise when the signal is very weak. The Hamamatsu camera was connected to a dedicated computer with a CamLink interface. However, although the Hamamatsu camera has a high sensitivity, it was not possible to measure small variations in the signal intensity. It turned out that the overall background intensity of the camera image varied on a frame by frame basis. This so called baseline drift problem was solved by using a different EMCCD camera. The Andor Luca DL658M is a cooled EMCCD camera that connects to the USB 2.0 bus. It has a baseline clamping feature that eliminates almost all baseline drift.

The linearity of all cameras was confirmed experimentally. We also recorded a background image for every experiment. For most experiments it was sufficient to subtract the average value of the background image from the measured signal. However, the experiments in Chapter 9 required a measurement of the exact intensity distribution over the whole camera. Therefore, the full background image was subtracted pixel by pixel. Moreover, in that experiment we corrected for the approximately $30 \%$ lower sensitivity of the camera close to the corners of the CCD chip. The lower sensitivity is probably the result of a minute misalignment of the microlenses on this chip.

\subsubsection{Timing}

In all our experiments it turned out that the speed at which the optimal wavefront can be constructed is the limiting factor. Therefore, we want to measure as quickly as possible. In Section 2.1.6 we saw that the wavefront oscillates with a period of $8.3 \mathrm{~ms}$. To avoid noise due to aliasing, the shutter time of the cameras $\left(T_{\text {meas }}\right)$ was always set to a multiple of this value.

In Section 2.1.6 we observed that it takes some time for the image on the LCD to start changing $\left(T_{\text {idle }}\right)$ and then it takes some more time for the image to stabilize 


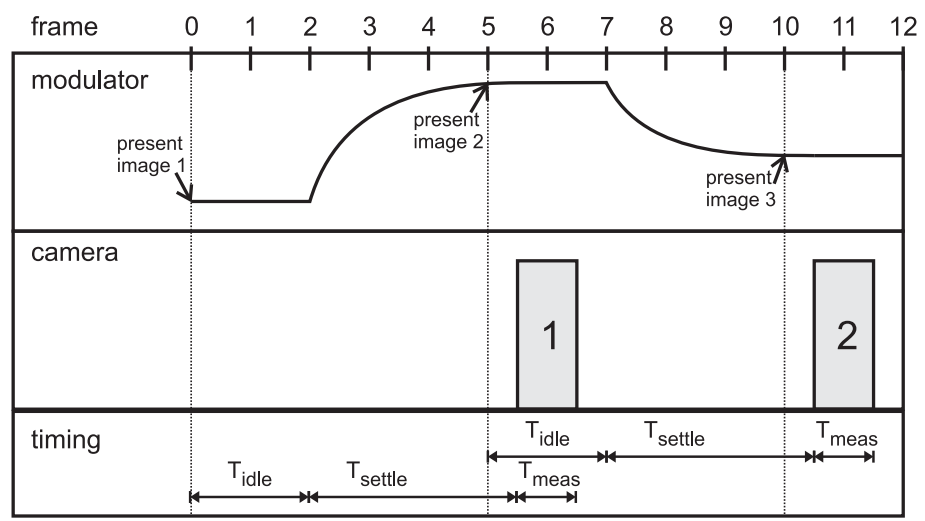

Figure 2.12: Timing diagram of a series of measurements. Time (in frames of $16.7 \mathrm{~ms}$ ) is indicated on the top axis. The curve in the topmost box symbolizes the switching behavior of the modulator. The numbered rectangles in the box below stand for the time that the camera is recording an image. In the lower box the relevant time intervals for synchronization are drawn.

( $T_{\text {settle }}$ ). To maximize the number of measurements per second and to further reduce aliasing effects, we synchronized the camera with the wavefront synthesizer.

A timing diagram of a series of measurements is given in Fig. 2.12. In this diagram, $T_{\text {idle }}=33 \mathrm{~ms}=2$ frames and $T_{\text {settle }}=57 \mathrm{~ms}=3.4$ frames. The first image is presented during the vertical retrace period of the modulator (frame 0 ). At frame 2 , the image starts to change. Then, at frame 5 , the second image is presented, although the measurement for the first image has not even started. Since it takes two more frames for the LCD to react, the image on the LCD is stable from frame 5 to frame 7 . We trigger the camera after $T_{\text {idle }}+T_{\text {settle }}=5.4$ frames. Even if there is some jitter in the timing, the camera has finished measuring before the image on the LCD starts changing (frame 7).

With this tight timing scheme, we can perform a measurement each 5 frames (instead of each 7 frames). The idle time $T_{\text {idle }}$ only affects the first measurement of a sequence. In the experiments we perform a sequence of 5 to 10 synchronized measurements for each segment.

\subsection{Stability}

The optimized wavefront is unique to a sample. When the sample is moved, the optimized wavefront does not fit the sample anymore. Therefore, during the course of an optimization, the sample has to be stable with sub-wavelength accuracy. The stability demands for the rest of the apparatus are high as well. In this section we discuss the most important stability considerations for our experiments.

The whole apparatus was built with the requirements of interferometric stability in mind. We built it on an actively levelled, damped optical table ${ }^{6}$. Moreover, only 
high quality opto-mechanical components were used 7 . The sample was mounted on a flexure stage to avoid the creep problems that are intrinsic to stages with ball bearings. The mechanical stability of the system was tested by tapping the optical components while monitoring the position of the focused light with sub-micron accuracy. If the focus did not return to exactly the same position after tapping a component, the mount of the component was replaced by a more stable one.

The temperature and humidity of the setup were not controlled. Therefore, it is likely that thermal drift and hygroscopic expansion negatively affect the stability. After switching on the system, it takes a few hours to stabilize completely. During this time the laser, the cameras, the LCD, and the computer controlled translation stages warm up. After this warmup time the total system is, typically, stable enough to perform an optimization that takes an hour.

We found that air turbulence causes fluctuations in the feedback signal. These fluctuations are in the order of a few percent in the interesting frequency range of about $1 \mathrm{~Hz}$. To reduce air turbulence, a box was built around the experiment. This box reduces stray light as well. As the laser is the most important source of hot, turbulent air, it needed to be placed outside the box. Of course, all components with fans (the cooled camera and the LCD control electronics) were also placed outside the box.

Finally, there is a constraint of the stability of the wavelength of the laser. To calculate the required stability, we estimate the average path length through a sample. For example: a typical sample has a mean free path of the path of $\ell=0.7 \mu \mathrm{m}$, and a thickness of $L=15 \ell$. Then, the diffuse path length $s$ is in the order of $s=15^{2} \ell=160 \mu \mathrm{m}$. At a wavelength of $532 \mathrm{~nm}$, a typical path is 300 wavelengths long. When a wavelength change results in a $\pi$ phase shift over this path length, the wavefront optimization fails. In this example, the wavelength needs to be stable to within a nanometer (or expressed in inverse centimeters, better than $31 \mathrm{~cm}^{-1}$ ), which is absolutely no problem for a temperature stabilized Nb:YAG laser or a HeNe laser.

\subsection{Samples}

For our experiments we used a large diversity of strongly scattering objects. In many ways, our method for controlling the propagation of light in such objects does not depend on the optical parameters of the sample. There are, however, some practical limitations that restrict what samples we can use:

Stability The optimal wavefront for focusing light through a strongly scattering object uniquely depends on the exact configuration of the scatterers. Therefore, we are limited to solid samples. We observed that after optimization the signal decreases with a typical timescale of approximately one hour, a value that is probably limited by thermal drift in the setup rather than by the samples.

Absorption For our method to work, there has to be at least some light on the detector before the optimization procedure is started. If the absorption in the

\footnotetext{
${ }^{7}$ Most opto-mechanical components were manufactured by Siskiyou and Thorlabs. For the beam expander, the laser, the LCDs, and the sample, special stable mounts were designed.
} 
sample is too strong, there will be no feedback signal to optimize. For most experiments it should be possible to use weakly absorbing materials as long as the initial transmission is detectable. However, for the experiment in Chapter 8, we expect that absorption decreases the effect that we are interested in. Therefore, we only used non-absorbing materials in all our experiments.

Thickness The optimization method detects the intensity in a single scattering channel. The number of independent channels is roughly equal to $(2 L)^{2} /(\lambda / 2)^{2}$, where $L$ is the thickness of the sample and $\lambda$ is the wavelength. Moreover, the total transmission scales as $\ell_{\text {tr }} / L$, with $\ell_{\text {tr }}$ the transport mean free path for light in the medium. Therefore, the total optical power in a single speckle scales with $L^{-3}$. For this reason, most of our samples were made relatively thin $(\sim 10 \mu \mathrm{m})$. However, successful optimizations have been performed on samples that were up to $1.5 \mathrm{~mm}$ thick (a baby tooth, see Table 3.1 ).

Scattering strength We want to study light propagation in non-absorbing opaque scattering media where all transmitted light is diffuse. A scattering object is opaque when it is thicker than a few transport mean free paths. We require that $L>4 \ell_{\mathrm{tr}}$. In this regime, the fraction of ballistic (non-diffuse) transmission is less than $\exp (-4) \approx 2 \%$. To keep the number of channels as low as possible, it is advantageous to use thin samples with a low $\ell_{\mathrm{tr}}$. There are two ways to minimize $\ell_{\text {tr }}$. First of all, the scale of the disorder should be comparable to the wavelength of light in the medium. Secondly, the index contrast should be as high as possible. Good candidates for making strongly scattering samples are pigment particles made of a high index material $\left(\mathrm{TiO}_{2}, \mathrm{ZnO}\right)$. These particles have a typical size of $\sim 200 \mathrm{~nm}$.

Flatness and homogeneity Our method for controlling propagation of light does not depend on the flatness or the homogeneity of the samples. However, for systematically analyzing the experimental results it is highly desirable to have samples that have the same thickness over the whole sample area $(<20 \%$ variations). Furthermore, the samples should be homogeneous in composition and certainly not have any holes.

Special requirement: doping For the experiment that is described in Chapter 5 it was required to place fluorescent nanospheres in the scattering medium.

Special requirement: substrate The experiment in Chapter 9 required that the samples were on a thin glass substrate to allow two high NA microscope objectives to focus both on the front and the back of the sample. Working without substrate all together was not possible because the substrate provides structural stability to the sample.

Special requirement: effective refractive index The experiment in Chapter 9 also required the number of channels to be as low as possible. Therefore, we used thin, strongly scattering samples. Moreover, we chose to use $\mathrm{ZnO}$ pigment in an air matrix because of its relatively low effective refractive index. A low refractive index reduces reflection at the sample boundaries and, thereby, reduces the size of the diffuse spot and the number of independent scattering channels. 
In conclusion, for most experiments the requirements on the samples are not very stringent and allow for a wide range of materials to be used. We successfully applied our wavefront shaping method to daisy petals, porous gallium phosphide, $\mathrm{TiO}_{2}$ pigment, $\mathrm{ZnO}$ pigment, white airbrush paint, eggshell, stacked layers of 3M Scotch tape, paper and even a baby tooth.

\subsubsection{Sample preparation method}

We developed a spray painting technique to fabricate layers of strongly scattering material. The technique allows the fabrication of flat, homogeneous layers with a thickness of around $5 \mu \mathrm{m}$ and more. Moreover, it is very easy to use different materials or to dope the sample with fluorescent markers. We first give the recipe for $\mathrm{ZnO}$ samples with embedded fluorescent nanospheres. These samples were used for the experiment in Chapter 5. Then we explain how different samples were made using the same technique.

1. Substrate cleaning Standard $40 \mathrm{~mm} \times 24 \mathrm{~mm}$ microscope cover slips with a thickness of $160 \mu \mathrm{m}$ were used as a substrate. The substrates were first rinsed with acetone to remove any residual organic material. Then they were rinsed with isopropanol, a solvent that is known to leave no drying stains, and left to dry.

2. Paint preparation First, the nanosphere suspension ${ }^{8}$ was diluted by a factor of $10^{5}$ $(2.5 \mu \mathrm{l}$ suspension in $250 \mathrm{ml}$ water). Then, a suspension was made by mixing $2.5 \mathrm{~g}$ of $\mathrm{ZnO}$ powder ${ }^{9}$ with $7.3 \mathrm{ml}$ water. The suspension was stirred on a roller bank for 30 minutes and then placed in an ultrasonic bath for 15 minutes. Finally, $0.73 \mathrm{ml}$ of the diluted nanosphere solution was added. The suspension was again stirred for 30 minutes and then placed in the ultrasonic bath for 15 seconds.

3. Spray painting The paint was sprayed onto the substrate with an airbrush ${ }^{10}$. The airbrush was operated at an air pressure of 2.3 bar. The paint was sprayed from approximately $20 \mathrm{~cm}$ distance to allow for a homogeneous coverage. The empty substrates were taped to an underground with an inclination of approximately $45^{\circ}$. Spraying covered these substrates with a thin wet film of paint. Directly after spraying, the samples were left to dry horizontally for two hours.

The resulting samples are homogeneous, flat up to a variation of $1 \mu \mathrm{m}$, and opaque (see Fig 2.13). The adhesion between the $\mathrm{ZnO}$ and the glass is remarkably good, especially since no binding agent was used at all. The thickness of the samples was determined around a scratch in the sample surface using optical microscopy or Dektak profilometry. At $\lambda=532 \mathrm{~nm}$, the mean free path is $0.7 \pm 0.2 \mu \mathrm{m}$, which was determined from total transmission measurements (see Section 5.A.3).

${ }^{8}$ Duke Scientific red fluorescent nanospheres. Diameter $0.30 \pm 5 \% \mu \mathrm{m}$. Suspension with $1 \%$ solids in water. $6.7 \cdot 10^{11}$ spheres / ml. Dyed with FireFly ${ }^{\mathrm{TM}}$ excitation maximum $542 \mathrm{~nm}$, emission maximum $612 \mathrm{~nm}$.

${ }^{9}$ Sigma-Aldrich Co. Zinc Oxide powder, $<1 \mu \mathrm{m}, 99.9 \% \mathrm{ZnO}$. With a scanning electron microscope (SEM) the average grain size was determined to be $200 \mathrm{~nm}$

${ }^{10}$ Evolution Solo Airbrush from Harder \& Steenbeck, $0.4 \mathrm{~mm}$ needle diameter. 
a

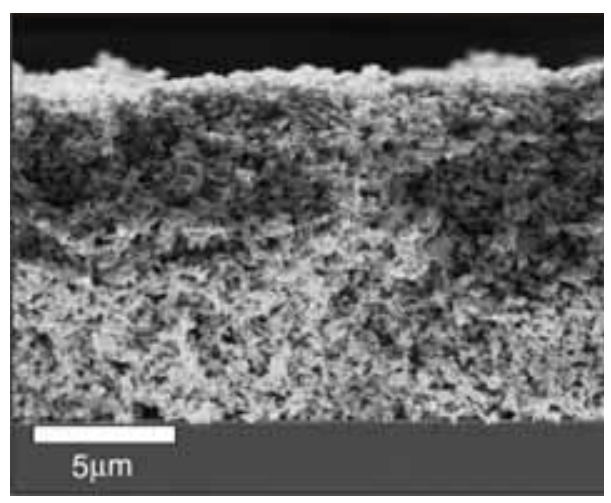

b

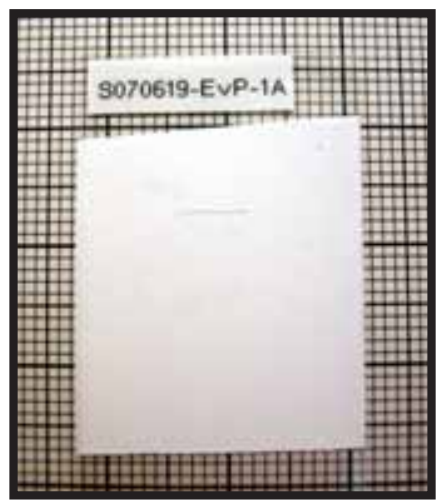

Figure 2.13: a) Scanning electron microscopy image of a $\mathrm{ZnO}$ sample (side view). Both the sample-air interface (top) and the sample-glass interface (bottom) are visible. b) Photograph of a $\mathrm{ZnO}$ sample (on a background of millimeter paper).

Various other suspensions were used to make samples. The most homogeneous samples were obtained with a mixture of 2.5 parts airbrush paint ${ }^{11}$ to 1 part acrylic medium ${ }^{12}$. We also created samples using a suspension of $5 \mathrm{~g}$ of rutile $\mathrm{TiO}_{2}$ pigment $^{13}$ in $10 \mathrm{ml}$ airbrush thinner. Both the samples with airbrush paint and the samples with rutile pigment are weakly fluorescent, which makes it hard to distinguish between the probe signal and the background fluorescence. Therefore, for the experiments in Chapter 5 we used $\mathrm{ZnO}$ samples, which do not fluoresce in the relevant wavelength area.

\subsection{Control program}

All elements of the experiment are controlled and synchronized by a control program. This program manages frame grabbing, detector triggering, video hardware acceleration, optimization algorithms, user input, realtime visual feedback, data storage and more. To keep a program of this scope maintainable we used a component based development strategy. Instead of thinking of the program as a whole, we separated it into self-contained components that offer a predefined service. An excellent introduction into the ideas behind of component based programming and agile software development can be found in Ref. 26.

Each component implements one or more interfaces. An interface is a specification of a set of properties (publicly accessible data) and methods (executable functions) with a certain functionality. For example, the interface of a camera component defines a method for triggering the camera, and it defines properties such as the shut-

\footnotetext{
${ }^{11}$ Hansa pro-color opaque white. Pigmented, water-based acrylic polymer paint. Pigment: $\mathrm{TiO}_{2} \mathrm{PW} 6$, CI\#77891 (specifications from manufacturer material safety data sheet).

${ }^{12}$ Schminke 50602 acrylic airbrush thinner

${ }^{13}$ Sachtleben Rutil R210 \#1060377/040
} 
ter time and the region of interest. Interfaces are defined in the most general terms possible and hide all details of the implementation.

The only way to interact with a component is through its external interface. This gives the possibility to create a different component that implements the same interface with different underlying details, for example using a different low-level camera driver. From the outside, these two components are indistinguishable. This means that we can exchange these two components transparently.[27]

The interchangeability of components also makes it possible to extend the functionality of code without changing it. This tantalizing idea is known as the OpenClosed principle[28]. For example, the first algorithm we wrote for shaping a wavefront was developed with a photodiode detector and a phase-mostly modulation configuration. Because of the abstraction that was used, we were able to extend this algorithm to work with an EMCCD camera and use the phase-amplitude modulation scheme simply by connecting two different components to the algorithm. Not a single line in the code of the algorithm was changed.

\subsubsection{LabView and $\mathrm{C}_{++}$mixed programming}

We chose a mixed programming environment for developing the control program. We used $\mathrm{C}++$ to implement reusable components for the detectors, algorithms and modulation schemes. Then, we used LabView to construct the measurement procedure for a specific experiment. The rationale behind this separation to make writing a measurement procedure as simple as possible.

LabView is a visual scripting environment that allows rapid development of simple measurement procedures. Moreover, it is very easy to create a user interface for setting the parameters of the experiment and for giving dynamic feedback in the form of graphs and false-color images. However, LabView is not suitable for creating complex programs or for performing time critical operations. Also, LabView code usually has a limited reusability.

$\mathrm{C}++$, on the other hand, is a programming language that provides a low level control over the hardware and allows for writing time critical code. Moreover, $\mathrm{C}++$ is a structured programming language that offers many mechanisms for code reuse and abstraction. Additionally, the Microsoft Visual Studio programming environment allows easy navigation through a complex project and contains various useful tools such as an excellent debugger.

\subsubsection{Component Object Model}

The Component Object Model (COM) is a standard for writing reusable components. A very accessible introduction to COM is given in Ref. 29. COM was introduced by Microsoft to enable interprocess communication and dynamic object creation in a way that is independent of the programming language that is used. Nowadays, COM and ActiveX (one of the extensions to COM), are supported on various operating systems.

COM/ActiveX components are listed in the Windows registry. The registry contains information about the location of the components executable code, as well as a description of the interface(s) of the component. In almost any modern programming 


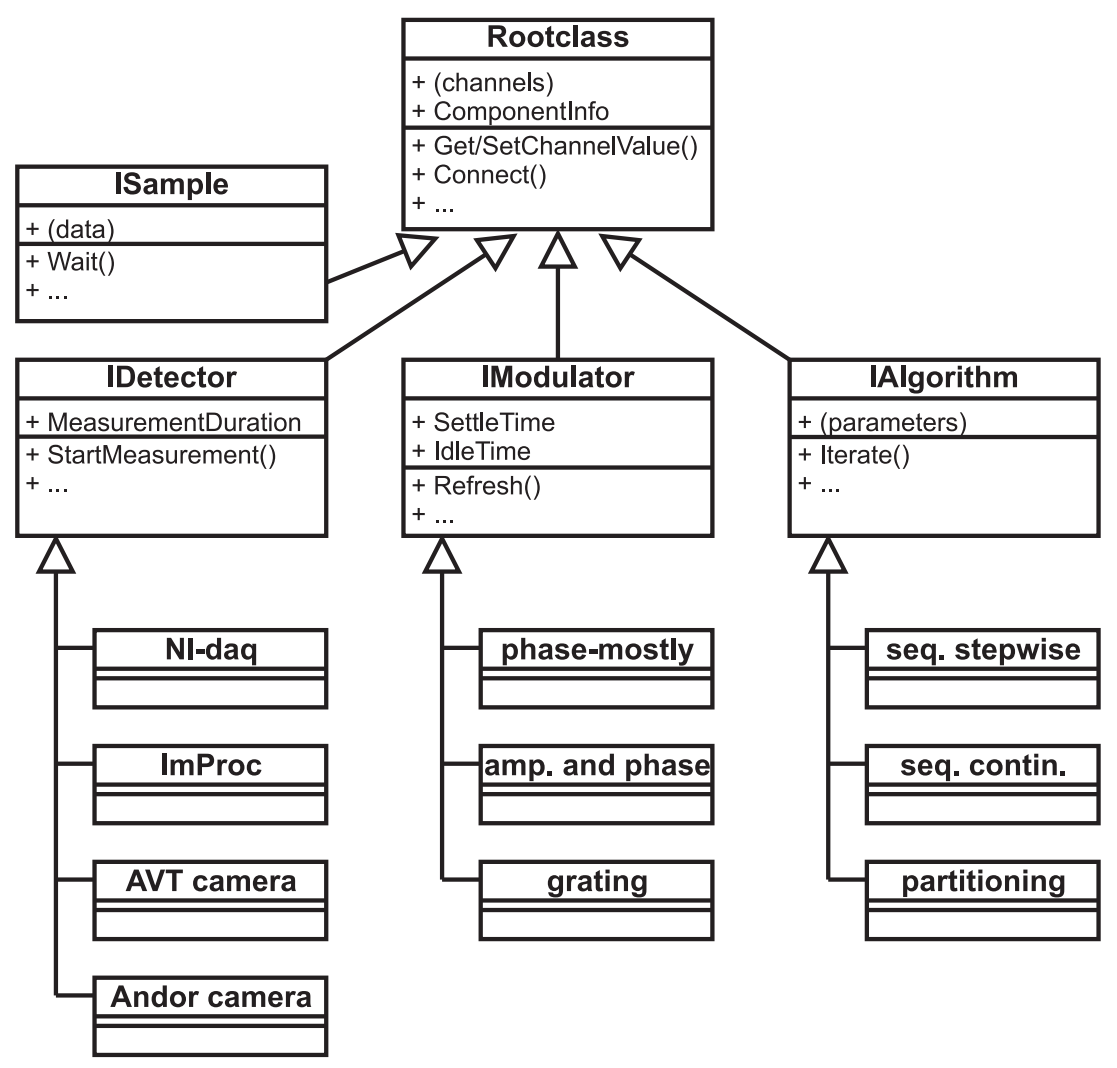

Figure 2.14: Simplified class diagram for the most important components of the control program. Blocks indicate classes. The top part of a box contains the class name, the middle part contains its properties and the lower part its methods. Arrows denote inheritance. E.g. AVT camera is an implementation of IDetector, which, in turn, derives from Rootclass.

language, including LabView, it is possible to select a component from the registry and use it as if it was written in that programming language. It is even possible create a COM object on a remote computer and access it as if it is a local object. The operating system makes sure that all function calls are transparently relayed over the network connection. We successfully used this feature to access the Hamamatsu EMCCD camera that was operated by a dedicated computer.

\subsubsection{Global structure of the control program}

We defined four interfaces for describing different functions in the control program. These interfaces are 'IDetector' (for accessing detectors and cameras), 'IModulator' (for programming the light modulator), 'IAlgorithm' (that implements a wavefront shaping algorithm) and 'ISample' (for storing and transferring measurement data). All interfaces have some common functionality that is embedded in a common root class. A simplified class diagram of the interface hierarchy is shown in Fig. 2.14. We 
first discuss these interfaces and then explain how these components are connected to form a measurement program.

Rootclass (IDataAccess) We constructed a common base interface for all components. It defines functions for accessing the component's data in a uniform way. All publicly accessible data of a component is organized in named 'channels'. Access to the data is, in many ways, independent of the data type. For example SetChannelValue("Shutter", 1000) sets the shutter time of a camera to $1000 \mu$ s and SetChanneIValue("Phase", 0) sets the phase of all segments on a modulator to 0 . Some data channels represent connections to a different component (more on this below).

The property ComponentInfo contains a text string with the description of the component, including the names and values of all data channels as well as the information of all connected components. All measurement programs save this string to the disk so that the values of all parameters in the experiment are stored.

IDetector and ISample The detector interface encompasses everything that returns a signal. Its channels correspond to settings of the detector, such as the shutter time for a camera. When StartMeasurement() is called, the detector returns a sample object containing all measured data. Data acquisition happens asynchronously: the sample object is returned before the measurement and the data processing is complete. Only when GetChannelValue() is called on one of the data channels of the sample the computer waits until that data is available. It is also possible to explicitly wait for the measurement to finish by calling Wait() on the sample.

The detector has a property MeasurementDuration, which is the maximum time that the measurement will take. This property is used for synchronizing the modulator and the measurements.

There are many implementations of the IDetector interface. We wrote a component for each of the types of cameras and for the National Instruments data acquisition hardware (NI-daq). We also wrote image processing components (ImProc) that analyze a camera image and return a scalar value. Such 'virtual detector' components are completely interchangeable with real detectors since they have the same interface.

IModulator The modulator interface standardizes a light modulator. A modulator component has channels that contain matrices for the phase and, depending on the modulation scheme, amplitude. The properties SettleTime and IdleTime affect the synchronization of the measurements (see Section 2.2.1). The function Refresh() is called to display the new image after the phase and/or amplitude channels have been changed.

For testing and characterization purposes, different types of modulator components have been created, such as the one that displays a Ronchi grating.

IAlgorithm The algorithm component contains code for the feedback procedure. The three different algorithms that are listed in Fig. 2.14 are discussed in Chapter 7. 


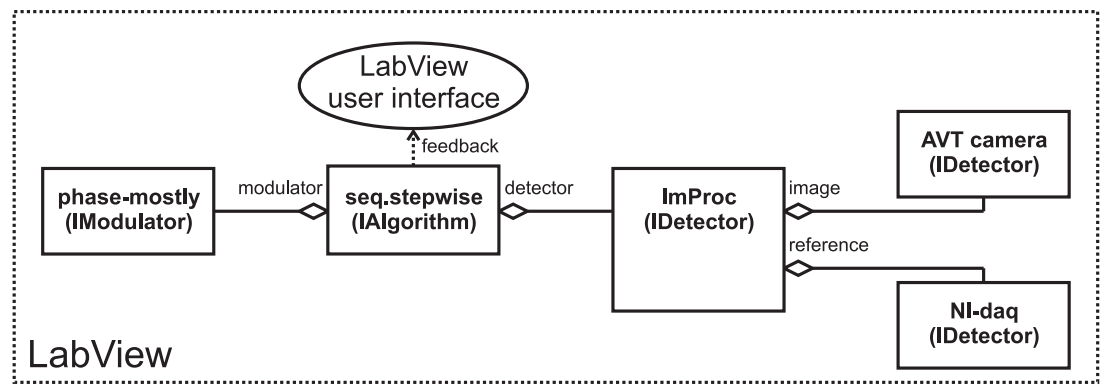

Figure 2.15: Structure of a typical wavefront optimization program. Rectangles, ActiveX components written in $\mathrm{C}++_{+}$are instantiated in a LabView environment and connected to each other; lines, connection between a component and a channel (diamond) on a different component; ellipse, a user interface constructed in LabView program provides feedback during optimization.

The algorithm is operated by connecting a detector and a modulator object and then repeatedly calling Iterate().

The LabView program instantiates the required objects and sets parameters such as the shutter time, the number of segments, the position of the target, etc. Then, the program connects the components together. The structure of a typical wavefront optimization program is shown in Fig. 2.15. A modulator and an image processing node are connected to the algorithm. The image processing node takes two inputs, one for the camera and one for a reference detector. The node averages the camera image over the target area and divides this value by the reference signal to compensate for variations in the incident intensity. The image processing node also takes care that the camera and the reference detector are triggered simultaneously. A LabView user interface displays the current status of the algorithm (percentage done, optimal wavefront settings measured so far, etc.).

It proved to be relatively easy to quickly build a program with these predefined components. Moreover, most of the LabView code could also be reused in different experiments by simply exchanging components. The design is open for extension; different cameras, algorithms, target functions and modulation schemes were added without affecting other parts of the program. All in all, the flexible design helped in quickly setting up an experiment.

\subsection{Conclusions and outlook}

In this chapter, we described the setup that was used for our wavefront shaping experiments. The complete apparatus was designed from scratch. We discussed the considerations that played a role in designing the experiment. Especially the wavefront synthesizer turned out to be more involved than the cartoon in Fig. 1.1 suggests. We first used a state-of-the-art method to achieve phase-mostly modulation with a commercial liquid crystal display. Then, we developed a new method that allows full 
control over both phase and amplitude of the light. We also discussed a versatile fabrication method that we developed for making samples. This method allowed us to make samples with different pigments and to embed fluorescent nanospheres.

We wrote a control program that executes the optimization algorithm and handles all data flow in the experiment. The program was designed using a component based development strategy. All detailed programming code was packaged in reusable and interchangeable components. Therefore, it was easy to quickly try alternative measurement schemes, to test new algorithms, and to replace detectors and cameras in a late stage of the project.

The setup that is discussed in this chapter is capable to create an optimal wavefront in a matter of minutes. Although this is an impressive achievement by itself, our method cannot yet be applied to media with rapidly moving scatterers such as perfused skin or turbid fluids. We believe that it is possible to speed up the measurements considerably. Currently the speed is limited by the response time of the LCD ( 50 ms). However, recently adaptive optics systems for turbulence correction have already been demonstrated to reach a correction speed of $800 \mathrm{~Hz}$.[30] Modulators that use ferroelectric liquid crystals (FLC) or transparent lanthanum-modified lead zirconate titanate (PLZT) as a switching medium can have a response time that is as short as $3 \mu \mathrm{s}$. Even faster switching is achieved with micromachined mirrors; such light modulators were shown to have a response frequency of up to $10 \mathrm{MHz}$.[31] Typical frequencies for a centimeter of living human tissue are in the order of 1 $100 \mathrm{kHz}[32,33]$ and we believe that these rates can be achieved with our method in the near future.

\section{Bibliography}

[1] E. G. van Putten, I. M. Vellekoop, and A. P. Mosk, Spatial amplitude and phase modulation using commercial twisted nematic LCDs, Appl. Opt. (2008), accepted. Preprint avaiable on arXiv: physics.optics/0711.4301. Also see Section 2.1 of this thesis.

[2] R. K. Tyson, Principles of adaptive optics, 2nd ed. (Academic Press, New York, 1998).

[3] D. Psaltis and G. Burr, Holographic data storage, Computer 31, 52 (1998).

[4] A. R. L. Travis, The display of three-dimensional video images, Proceedings of the IEEE $\mathbf{8 5}$, 1817 (1997).

[5] J. A. Davis, D. E. McNamara, and D. M. Cottrell, Encoding complex diffractive optical elements onto a phase-only liquid-crystal spatial light modulator, Optical Engineering 40, 327 (2001).

[6] S.-T. Wu and D.-K. Yang, Reflective liquid crystal displays (Wiley, Chichester, 2001).

[7] Z. Zhang, G. Lu, and F. T. S. Yu, Simple method for measuring phase modulation in liquid crystal televisions, Optical Engineering 33, 3018 (1994).

[8] J. Reményi, P. Várhegyi, L. Domján, P. Koppa, and E. Lõrincz, Amplitude, phase, and hybrid ternary modulation modes of a twisted-nematic liquid-crystal display at $400 \mathrm{~nm}$, Appl. Opt. 42, 3428 (2003).

[9] O. Aso, I. Ohshima, and H. Ogoshi, Unitarity-conserving construction of the Jones matrix and its application to polarization-mode dispersion analysis, J. Opt. Soc. Am. A 14, 1998 (1997).

[10] J. A. Davis, I. Moreno, and P. Tsai, Polarization eigenstates for twisted-nematic liquidcrystal displays, Appl. Opt. 37, 937 (1998).

[11] C. R. Fernández-Pousa, I. Moreno, N. Bennis, and C. Gómez-Reino, Generalized formula- 
tion and symmetry properties of reciprocal nonabsorbing polarization devices: application to liquid-crystal displays, J. Opt. Soc. Am. A 17, 2074 (2000).

[12] N. Konforti and E. Marom, Phase-only modulation with twisted nematic liquid-crystal spatial light modulators, Opt. Lett. 13, 251 (1988).

[13] A. Lien, Extended Jones matrix representation for the twisted nematic liquid-crystal display at oblique incidence, Appl. Phys. Lett. 57, 2767 (1990).

[14] M. Yamauchi and T. Eiju, Optimization of twisted nematic liquid crystal panels for spatial light phase modulation, Opt. Com. 115, 15 (1995).

[15] A. Bergeron, J. Gauvin, F. Gagnon, D. Gingras, H. H. Arsenault, and M. Doucet, Phase calibration and applications of a liquid-crystal spatial light modulator, Appl. Opt. 34, 5133 (1995).

[16] R. Dou and M. K. Giles, Phase measurement and compensation of a wave front using a twisted nematic liquid-crystal television, Appl. Opt. 35, 3647 (1996).

[17] J. A. Davis, J. Nicolás, and A. Márquez, Phasor analysis of eigenvectors generated in liquidcrystal displays, Appl. Opt. 41, 4579 (2002).

[18] S. Chavali, P. M. Birch, R. Young, and C. Chatwin, Synthesis and reconstruction of computer generated holograms by a double pass technique on a twisted nematic-based liquid crystal spatial light modulator, Optics and Lasers in Engineering 45, 413 (2007).

[19] L. G. Neto, D. Roberge, and Y. Sheng, Programmable optical phase-mostly holograms with coupled-mode modulation liquid-crystal television, Appl. Opt. 34, 1944 (1995).

[20] T.-L. Kelly and J. Munch, Phase-aberration correction with dual liquid-crystal spatial light modulators, Appl. Opt. 37, 5184 (1998).

[21] P. Birch, R. Young, D. Budgett, and C. Chatwin, Two-pixel computer-generated hologram with a zero-twist nematic liquid-crystal spatial light modulator, Optics Letters 25, 1013 (2000).

[22] V. Arrizón, Complex modulation with a twisted-nematic liquid-crystal spatial light modulator: double-pixel approach, Opt. Lett. 28, 1359 (2003).

[23] V. Bagnoud and J. D. Zuegel, Independent phase and amplitude control of a laser beam by use of a single-phase-only spatial light modulator, Opt. Lett. 29, 295 (2004).

[24] C. Hsueh and A. Sawchuk, Computer-generated double-phase holograms, Applied Optics 17, 3874 (1978).

[25] Aurora Systems, Inc., Method for modulating a multiplexed pixel display, US-patent 6067065s, 2000.

[26] R. C. Martin, Agile software development, principles, patterns, and practices (Prentice Hall, Upper Saddle River, NJ, 2003).

[27] B. H. Liskov and J. M. Wing, A behavioral notion of subtyping, ACM T. Progr. Lang. Sys. 15, 1811 (1994).

[28] R. C. Martin, The open-closed principle, C++ Report, SIGS 8, 1 (1996).

[29] K. Brockschmidt, Inside OLE (Microscoft Press, Redmond, WA, 1995).

[30] K. L. Baker et al., High-speed horizontal-path atmospheric turbulence correction with a large-actuator-number microelectromechanical system spatial light modulator in an interferometric phase-conjugation engine, Opt. Lett. 29, 1781 (2004).

[31] G. Griffith, B. Haji-saeed, S. K. Sengupta, W. D. Goodhue, J. Khoury, C. L. Woods, and J. Kierstead, Patterned multipixel membrane mirror MEMS optically addressed spatial light modulator with megahertz response, IEEE Phot. Tech. Lett. 19, 173 (2007).

[32] B. J. Vakoc, S. H. Yun, J. F. de Boer, G. J. Tearney, and B. E. Bouma, Phase-resolved optical frequency domain imaging, Optics Express 13, 5483 (2005).

[33] J. Li, G. Dietsche, D. Iftime, S. E. Skipetrov, G. Maret, T. Elbert, B. Rockstroh, and T. Gisler, Noninvasive detection of functional brain activity with near-infrared diffusing-wave spectroscopy, J. Biomed. Opt. 10, 044002 (2005). 


\title{
Chapter 3
}

\section{Focusing coherent light through opaque strongly scattering media}

\begin{abstract}
We report focusing of coherent light through opaque scattering materials, by control of the incident wavefront. The multiply scattered light forms a focus that is up to a factor 1000 brighter than the normal diffuse transmission.

[This chapter has been published as: I. M. Vellekoop and A. P. Mosk, Opt. Lett. 32, 2309-2311 (2007)]
\end{abstract}

Random scattering of light is what makes materials such as white paint, milk, or human tissue, opaque. In these materials, repeated scattering and interference distort the incident wavefront so strongly that all spatial coherence is lost.[1] Incident coherent light diffuses through the medium and forms a volume speckle field which has no correlations on a distance larger than the wavelength of light. The complete scrambling of the field makes it impossible to control light propagation using the well established wavefront correction methods of adaptive optics (see e.g. [2]).

We demonstrate focusing of coherent light through disordered scattering media by the construction of wavefronts that invert diffusion of light. Our method relies on interference and is universally applicable to scattering objects regardless of their constitution and scattering strength. We envision that, with such active control, random scattering will become beneficial, rather than detrimental, to imaging[1] and communication[3-5].

Figure 3.1 shows the principle of the experiment. Normally, incident light is scattered by the sample and forms a random speckle pattern (Fig. 3.1a). The goal is to
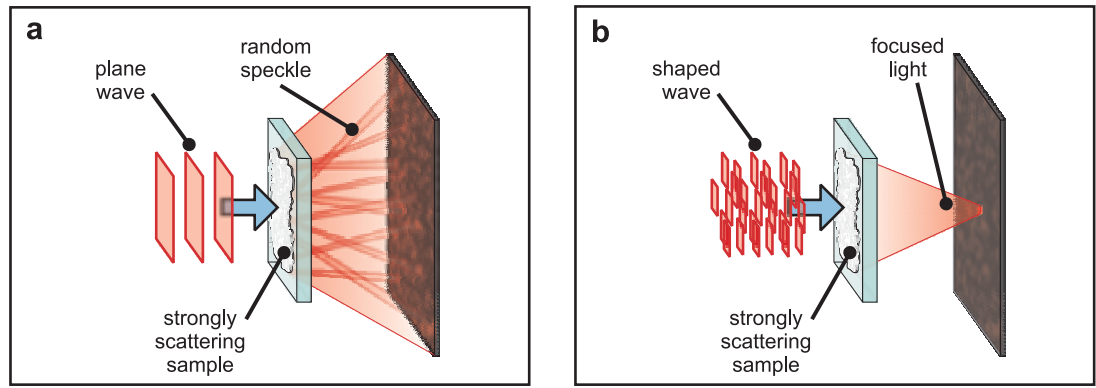

Figure 3.1: Design of the experiment. a) A plane wave is focused on a disordered medium, a speckle pattern is transmitted. b) The wavefront of the incident light is shaped so that scattering makes the light focus at a predefined target. 


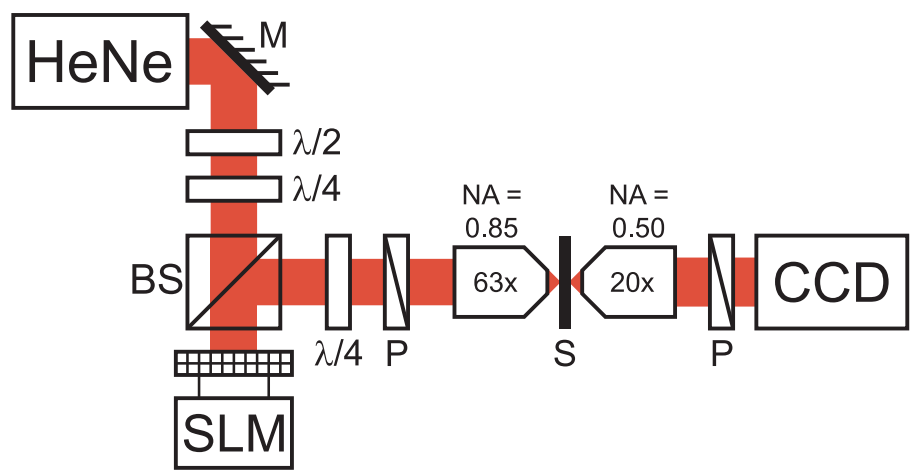

Figure 3.2: Schematic of the apparatus. A $632.8 \mathrm{~nm}$ HeNe laser beam is expanded and reflected off a Holoeye LC-R 2500 liquid crystal spatial light modulator (SLM). Polarization optics select a phase-mostly modulation mode. The SLM is imaged onto the entrance pupil of the objective with a 1:3 demagnifying lens system (not shown). The objective is overfilled; we only use segments that fall inside the pupil. The shaped wavefront is focused on the strongly scattering sample (S) and a CCD-camera images the transmitted intensity pattern. $\lambda / 4$, quarter wave plate. $\lambda / 2$, half wave plate. $M$, mirror. $B S, 50 \%$ non-polarizing beam splitter. P, polarizer.

match the incident wavefront to the sample, so that the scattered light is focused in a specified target area (Fig. 3.1b).

\subsection{Experiment}

The experimental setup for constructing such wavefronts is shown in Figure 3.2. Light from a $632.8 \mathrm{~nm}$ HeNe laser is spatially modulated by a liquid crystal phase modulator and focused on an opaque, strongly scattering sample. The number of degrees of freedom of the modulator is reduced by grouping pixels into a variable number $(N)$ of square segments. A charge coupled device (CCD) camera monitors the intensity in the target focus and provides feedback for an algorithm that programs the phase modulator.

We performed first tests of inverse wave diffusion using rutile $\left(\mathrm{TiO}_{2}\right)$ pigment, which is one of the most strongly scattering materials known. The sample consists of an opaque, $10.1 \mu \mathrm{m}$ thick layer of rutile[6] with a transport mean free path of $0.55 \pm 0.10 \mu \mathrm{m}$ measured at $\lambda=632.8 \mathrm{~nm}$. Since in this sample the transmitted light is scattered hundreds of times, there is no direct relation between the incident wavefront and the transmitted image.[7, 8]

In Fig. 3.3 we show the intensity pattern of the transmitted light. In Fig. 3.3a we see the pattern that was transmitted when a plane wave was focused onto the sample. The light formed a typical random speckle pattern with a low intensity. We then optimized the wavefront so that the transmitted light focused to a target area with the size of a single speckle. The result for a wavefront composed of 3228 individually controlled segments is seen in Fig. 3.3b, where a single bright spot stands out clearly 

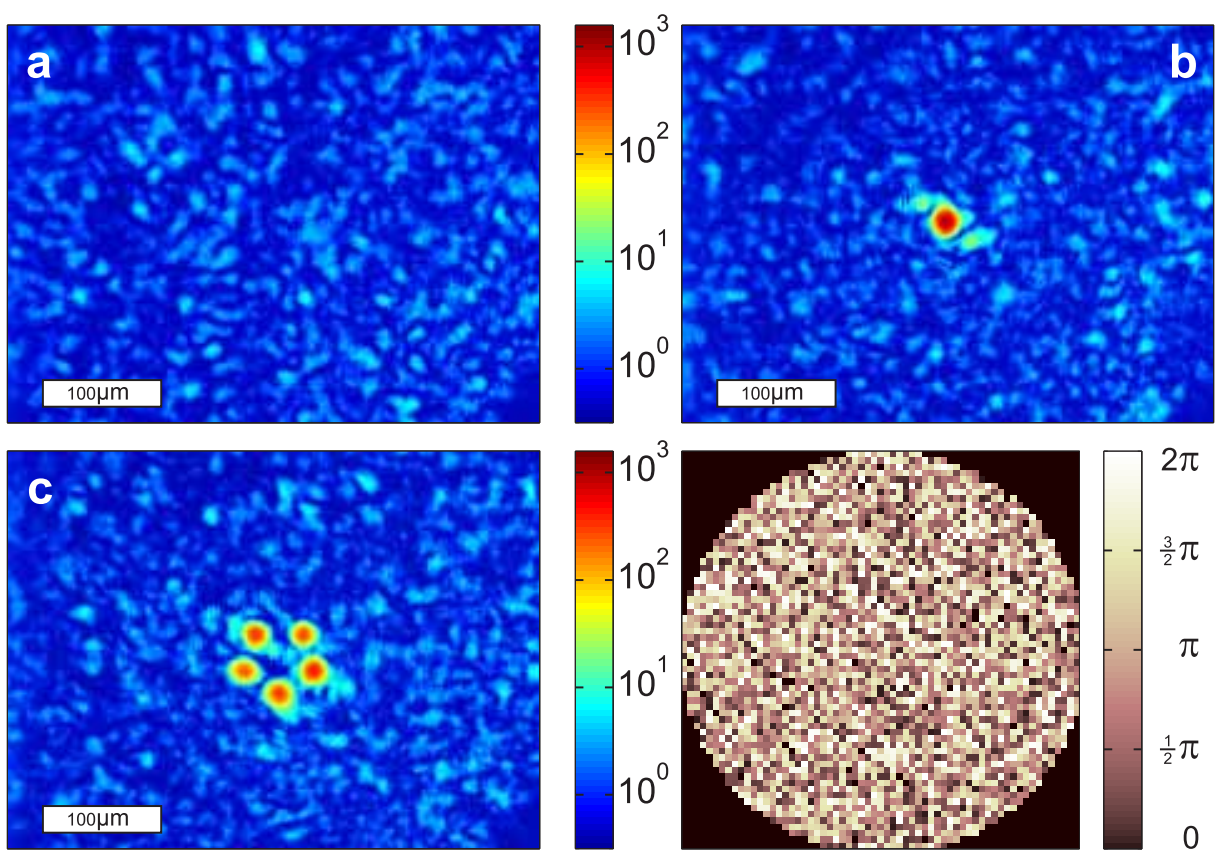

$2 \pi$

$\frac{3}{2} \pi$

$\pi$

$\frac{1}{2} \pi$

Figure 3.3: Transmission through a strongly scattering sample consisting of $\mathrm{TiO}_{2}$ pigment. a) Transmission micrograph with an unshaped incident beam. b) Transmission after optimization for focusing at a single target. The scattered light is focused to a spot that is 1000 times brighter than the original speckle pattern. c) Multi-beam optimization. The disordered medium generates five sharp foci at the defined positions. Figures $3.3 \mathrm{a}$ to $3.3 \mathrm{c}$ are presented on the same logarithmic color scale that is normalized to the average transmission before optimization. d) Phase of the incident wavefront used to form Fig. 3.3c.

against the diffuse background. The focus was over a factor 1000 more intense than the non-optimized speckle pattern. By adjusting the target function used as feedback it is also possible to optimize multiple foci simultaneously, as is shown in Fig. 3.3c where a pattern of five spots was optimized. Each of the spots has an intensity of approximately 200 times the original diffuse intensity. In Fig. 3.3d we show the phase of the incident wavefront corresponding to Fig. 3.3c. Neighboring segments are uncorrelated, which indicates that the sample fully scrambles the incident wavefront.

\subsection{Algorithm}

The algorithm that constructs the inverse diffusion wavefront uses the linearity of the scattering process. The transmitted field in the target, $E_{m}$, is a linear combination of the fields coming from the $N$ different segments of the modulator,

$$
E_{m}=\sum_{n=1}^{N} t_{m n} A_{n} e^{i \phi_{n}}
$$




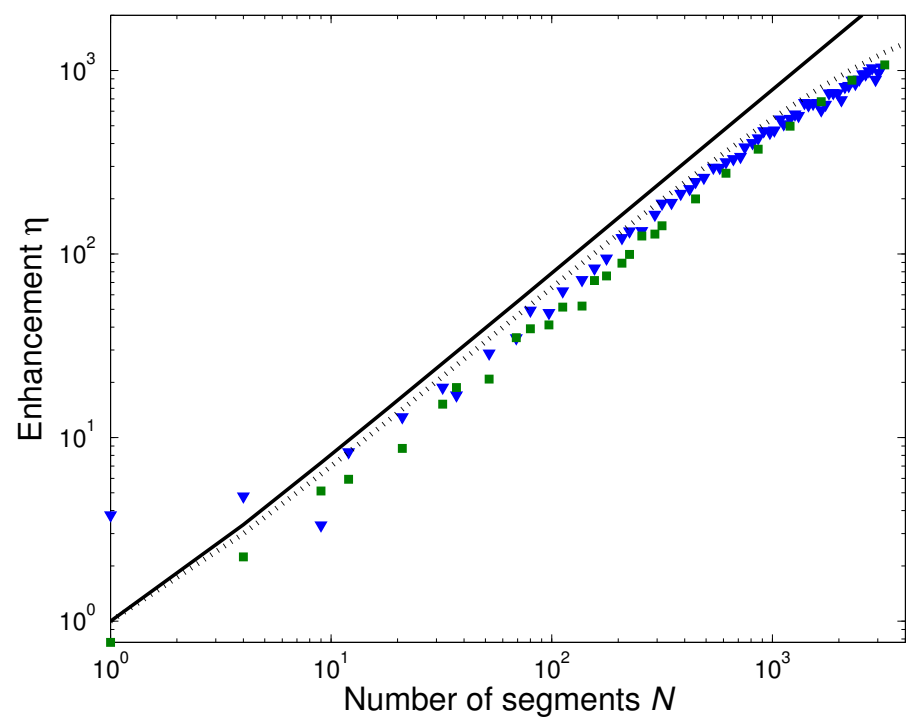

Figure 3.4: Measured intensity enhancement as a function of the number of segments. Squares, sample in focus, triangles, sample $100 \mu \mathrm{m}$ behind focus, solid line, ideal enhancement (Eq. (3.2)), dotted line, corrected for residual amplitude modulation and finite persistence time of $T_{p}=5400 \mathrm{~s}$. The experimental uncertainty is of the order of the symbol size.

where $A_{n}$ and $\phi_{n}$ are, respectively, the amplitude and phase of the light reflected from segment $n$. Scattering in the sample and propagation through the optical system is described by the elements $t_{m n}$ of the unknown transmission matrix. Clearly, the magnitude of $E_{m}$ will be the highest when all terms in Eq. (3.1) are in phase. We determine the optimal phase for a single segment at a time by cycling its phase from 0 to $2 \pi$. For each segment we store the phase at which the target intensity is the highest. At that point the contribution of the segment is in phase with the already present diffuse background. After the measurements have been performed for all segments, the phase of the segments is set to their stored values. Now the contributions from all segments interfere constructively and the target intensity is at the global maximum. A pre-optimization with a small number of segments significantly improves the signal to noise ratio. This method is generally applicable to linear systems and does not rely on time reversal symmetry or absence of absorption. Although mathematically this algorithm is the most efficient, in noisy experimental conditions adaptive learning algorithms[9] might be more effective and an investigation in such algorithms is on its way (also see Chapter 7).

\subsection{Scaling of the enhancement}

The maximum intensity enhancement that can be reached is related to the number of segments that are used to describe the incident wavefront. For a disordered medium the constants $t_{m n}$ are statistically independent and obey a circular Gaus- 
sian distribution $[8,10-12]$ and the expected enhancement $\eta$ - defined as the ratio between the optimized intensity and the average intensity before optimization - can be calculated,

$$
\eta=\frac{\pi}{4}(N-1)+1
$$

It was assumed that all segments of the phase modulator contribute equally to the total incident intensity. We expect the linear scaling behavior to be universal as Eq. (3.2) contains no parameters. Also, since we are free to choose the basis for Eq. (3.1), we expect to find the same enhancement regardless of whether the target is a focus or a far-field beam and regardless of how the shaped wavefront is projected onto the sample. Interesting correlations between the transmission matrix elements, which will cause corrections on Eq. (3.2), are predicted when $\mathrm{N}$ approaches the total number of mesoscopic channels.[11, 12] With our current apparatus we are far from this regime and no deviation from Eq. (3.2) is expected.

We tested the universal scaling behavior implied by Eq. (3.2) by changing $N$. Using the same $\mathrm{TiO}_{2}$ sample as before, the algorithm was targeted to construct a collimated beam. In Fig. 3.4 the enhancement is plotted as a function of the number of segments for different focusing conditions. The linear relation between the enhancement and the number of segments is evident until the enhancement saturates at $\eta=1000$. All measured enhancements were slightly below the theoretical maximum. This is understandable since all perturbations move the system away from the global maximum. The main reason for deviations from the optimal wavefront is residual amplitude modulation in the phase modulator, which introduced an uncontrolled bias in the field amounting to $14 \%$ of the total intensity.

The saturation of the enhancement is the result of slow changes in the speckle pattern. This instability effectively limited the number of segments for which the optimal phase could be measured. We estimate that the effective enhancement decreases to $\eta_{\text {eff }}=\eta /\left(1+N T / T_{p}\right)$, where $T=1.2 \mathrm{~s}$ is the time needed for one measurement and the persistence time $T_{p}=5400 \mathrm{~s}$ is the timescale at which the speckle pattern of the $\mathrm{TiO}_{2}$ sample remains stable. Depending on the environmental conditions, $T_{p}$ can be considerably higher and enhancements of over two thousand have been measured overnight.

To verify the universal applicability of inversion of wave diffusion, we used a variety of materials of natural origin. Table 3.1 lists the intensity enhancement for different materials we used. Although the samples vary in thickness, composition and scattering strength, they were all able to focus a properly prepared wavefront to a sharp spot. The intensity enhancement varies between 60 and 1000. The main reason for this variation is that the persistence time is not the same for all materials.

\subsection{Conclusion}

In summary, our results show that precise control of diffuse light is possible using an optimal, non-iterative algorithm; light can be directed through opaque objects to form one or multiple foci. The brightness of the focal spot is explained by a model based on statistical optics. We expect inverse wave diffusion to have applications 


\begin{tabular}{cccc}
\hline Sample & $\mathrm{L}(\mu \mathrm{m})$ & $\eta( \pm 15 \%)$ & $N$ \\
\hline Rutile $\mathrm{TiO}_{2}$ & $10.1 \pm 0.3$ & 1080 & 3228 \\
Daisy petal, fresh & $43 \pm 5$ & 64 & 208 \\
Daisy petal, dried & $37 \pm 5$ & 630 & 1664 \\
Chicken eggshell & $430 \pm 30$ & 250 & 3228 \\
Human tooth & $1500 \pm 100$ & 70 & 208 \\
\hline
\end{tabular}

Table 3.1: Measured intensity enhancement for different materials. $L$, sample thickness $( \pm$ surface roughness); $\eta$, maximum enhancement reached; $N$, number of segments used by the algorithm to describe the wavefront.

in imaging and light delivery in scattering media, possibly including metal nanostructures[13]. Dynamic measurements in biological tissue are possible when the time required for achieving a focus can be reduced to below $1 \mathrm{~ms}$ per segment[14, 15]; we estimate that this timescale is technologically possible with the use of fast phase modulators[16]. Furthermore, the high degree of control over the scattered light should permit experimental verification of random matrix theories for the transport of light $[11,12]$.

\section{Bibliography}

[1] Waves and imaging through complex media, edited by P. Sebbah (Kluwer Academic, Dordrecht, Netherlands, 2001).

[2] R. K. Tyson, Principles of adaptive optics, 2nd ed. (Academic Press, New York, 1998).

[3] A. Derode, A. Tourin, J. de Rosny, M. Tanter, S. Yon, and M. Fink, Taking advantage of multiple scattering to communicate with time-reversal antennas, Phys. Rev. Lett. 90, 014301 (2003).

[4] S. H. Simon, A. L. Moustakas, M. Stoytchev, and H. Safar, Communication in a disordered world, Phys. Today 54, 38 (2001).

[5] G. Lerosey, J. de Rosny, A. Tourin, and M. Fink, Focusing beyond the diffraction limit with far-field time reversal, Science 315, 1120 (2007).

[6] R. H. J. Kop, P. de Vries, R. Sprik, and A. Lagendijk, Observation of anomalous transport of strongly multiple scattered light in thin disordered slabs, Phys. Rev. Lett. 79, 4369 (1997).

[7] R. Pappu, B. Recht, J. Taylor, and N. Gershenfeld, Physical one-way functions, Science 297, 2026 (2002).

[8] J. W. Goodman, Statistical optics (Wiley, New York, 2000).

[9] D. E. Goldberg, Genetic algorithms in search, optimization \& machine learning (AddisonWesley, Reading, MA, 1989).

[10] N. Garcia and A. Z. Genack, Crossover to strong intensity correlation for microwave radiation in random media, Phys. Rev. Lett. 63, 1678 (1989).

[11] C. W. J. Beenakker, Random-matrix theory of quantum transport, Rev. Mod. Phys. 69, 731 (1997).

[12] J. B. Pendry, A. MacKinnon, and P. J. Roberts, Universality classes and fluctuations in disordered systems, Proc. R. Soc. Lond. A 437, 67 (1992). 
[13] M. I. Stockman, S. V. Faleev, and D. J. Bergman, Coherent control of femtosecond energy localization in nanosystems, Phys. Rev. Lett. 88, 067402 (2002).

[14] B. J. Vakoc, S. H. Yun, J. F. de Boer, G. J. Tearney, and B. E. Bouma, Phase-resolved optical frequency domain imaging, Optics Express 13, 5483 (2005).

[15] J. Li, G. Dietsche, D. Iftime, S. E. Skipetrov, G. Maret, T. Elbert, B. Rockstroh, and T. Gisler, Noninvasive detection of functional brain activity with near-infrared diffusing-wave spectroscopy, J. Biomed. Opt. 10, 044002 (2005).

[16] M. Hacker, G. Stobrawa, R. Sauerbrey, T. Buckup, M. Motzkus, M. Wildenhain, and A. Gehner, Micromirror SLM for femtosecond pulse shaping in the ultraviolet, Appl. Phys. B 76, 711 (2003). 
50 | Bibliography 


\section{Chapter 4}

\section{The focusing resolution of opaque lenses}

In this chapter, we investigate both experimentally and theoretically the focusing resolution of the opaque lenses that were introduced in Section 1.1. For every optical system there is a limit on how sharp it can focus light. The smallest focus that can theoretically be achieved with an aberration free lens is called the diffraction limit. The diffraction limit is determined by the aperture and focal distance of the lens. Here we examine how these concepts translate to the situation of an opaque lens. We use the experimental geometry that is shown in Fig. 4.1 to measure the focal width of an opaque lens and then compare this width to the theoretical diffraction limit of an ordinary lens.

In Section 4.1 we explain how scattering inside an opaque lens helps focusing. The focusing resolution of an opaque lens is determined experimentally in Section 4.2. In Section 4.3 we compare the shape of the focus with the measured speckle correlation function. To theoretically understand the focusing capacity of opaque lenses, a matrix model does not suffice. Therefore, we developed a continuous field description of opaque lenses in Section 4.4. This new theoretical framework explains the experimental results. Moreover, the theory predicts the existence of the optical equivalent

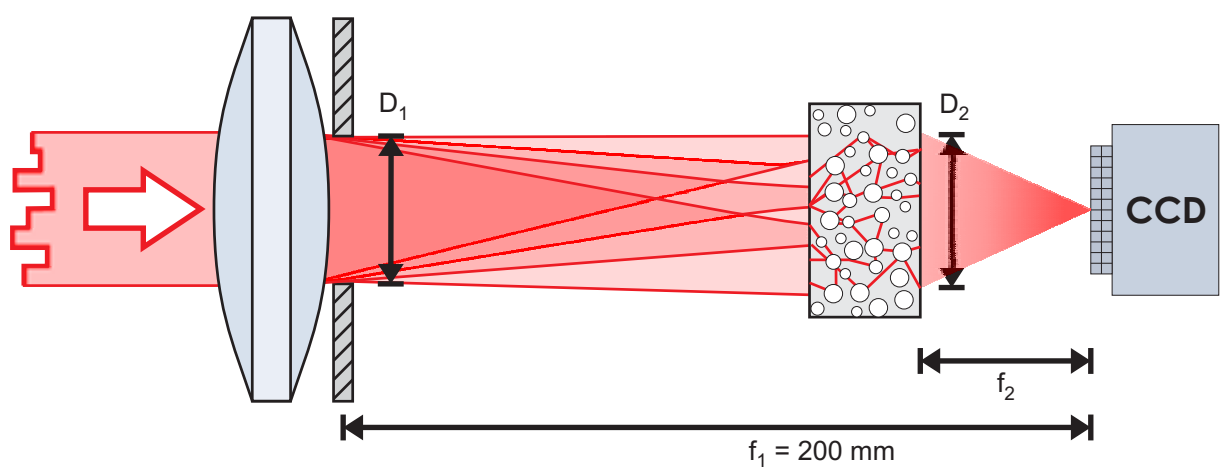

Figure 4.1: Schematic of the experimental geometry. Light from a phase modulator is imaged on the center plane of a lens (imaging telescope not shown). The numerical aperture of the lens is controlled by a pinhole. A CCD camera is positioned in the focal plane of the lens. Between the camera and the lens a strongly scattering sample is placed. The sample can be moved to change the distance to the camera. 


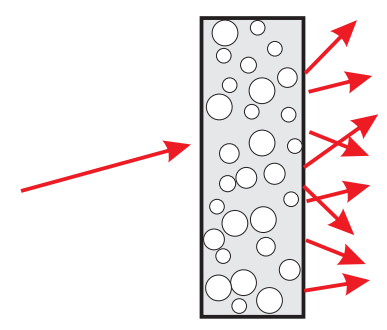

Figure 4.2: Scattering helps wavefront shaping and focusing. A single incident beam contributes to all transmitted wave vectors. As a result, a sharp focus can be generated even if the wavefront synthesizer only controls light in a small solid angle.

of the acoustical time-reversal lenses that focus sound beyond the diffraction limit[1].

\subsection{Wavefront shaping with an opaque lens}

In practice, it is extremely difficult to design (systems of) lenses that have almost no aberrations. [2, 3] A good microscope objective, for instance, contains up to twenty lens elements for compensating various aberrations.[4] An alternative approach to achieve a diffraction limited focus is to use adaptive optics to compensate for the aberrations in the lens system.[5] However, the adaptive optics system that generates the incident wavefront may have many limitations, such as a maximum number of control segments, residual phase-amplitude cross-modulation, a limited maximal wavefront curvature, etcetera. All such limitations cause systematical deviations from the diffraction limited focus.

We found that, surprisingly, the shape of the focus of an opaque lens is not affected by experimental limitations of the wavefront modulator. Paradoxically, the scattering medium makes that we have apparently more control over the scattered field than we have over the incident field. A similar effect has been observed in time-reversal experiments using ultrasound and microwaves: a limited number of broadband transmitters suffices to generate a diffraction limited focus through a disordered medium (see [6] for an excellent review on time-reversal focusing). Although our experiments are performed at a single (optical) frequency, many of the concepts developed for time-reversal experiments are still valid.

In Fig. 4.2, we illustrate how scattering improves focusing. Disordered scattering in the sample randomly redistributes the incident light over all scattering channels (see e.g. [7-9]). In a microwave experiment, it was demonstrated that even evanescent channels are excited.[10] Scattering makes that light from a single segment of the incident beam contributes to all wave vectors of the desired field. By tuning the relative phases of all segments, their contributions to the desired field add up constructively. The more segments are used, the better the desired field can be approximated. The constructed field will never be perfect as the number of degrees of freedom in the transmitted field cannot exceed the number of control segments. However, thanks to the disordered scattering any deviation in the generated field is, on average, uniformly distributed over all outgoing channels, resulting in a low overall intensity con- 
tribution. Imperfections in the generated wavefront cause a loss of contrast with the speckle background.

\subsection{Measured focusing resolution of an opaque lens}

The experimental geometry used for analyzing the focus of an opaque lens is shown in Fig. 4.1. The sample is a $6 \mu \mathrm{m}$ layer of airbrush paint on a standard microscope cover slip. The wavefront illuminating the sample is shaped to increase the intensity on a single pixel of the camera. The algorithm that shapes the wavefront is the same as was used in Chapter 3; it does not take into account the shape of the focus. The distance between the sample and the camera can be varied to analyze the focusing resolution of the opaque lens at different focal lengths.

We compare the measured focal width to the calculated diffraction limit. The optimal focus of a homogeneously illuminated lens with a circular aperture is an Airy disk.[2] The half width at half maximum of the Airy disk is $w=0.51 \lambda / \mathrm{NA}$, where NA is the numerical aperture of the lens. This radius is almost equal to the Abbe diffraction limit[11] of $w=\lambda /(2 \mathrm{NA})$. To calculate the diffraction limit of the opaque lens, we need to determine its numerical aperture $\mathrm{NA}_{2}=D_{2} /\left(2 f_{2}\right)$, where $D_{2}$ is the diameter of the illuminated area on sample and $f_{2}$ is the focal length of the opaque lens.

The diameter of the spot on the sample depends on what wavefront is generated. When the glass lens in front of the aperture is illuminated with a plane wave, the diameter of the spot on the sample equals $D_{2}=2 f_{2} \mathrm{NA}_{1}$, where the numerical aperture of the glass lens is $\mathrm{NA}_{1}=D_{1} /\left(2 f_{1}\right)=5 \cdot 10^{-3}$ with $D_{1}=2.1 \mathrm{~mm}$ the diameter of the aperture and $f_{1}=200 \mathrm{~mm}$ the focal length of the lens.

When the modulator is programmed to generate a shaped wavefront, diffraction at the lens aperture will cause the beam to diverge. Segments of the phase modulator are imaged onto the aperture to squares with sides of length $D_{s}=0.03 \mathrm{~mm}$. The angle of diffraction for a square source equals $\alpha \equiv(4 \lambda) /\left(\pi^{2} D_{s}\right)=0.008 \mathrm{rad}$ (see e.g. [12]). Combining the effects of the focusing and diffraction, we find the spot diameter

$$
D_{2}=2 f_{2} \mathrm{NA}_{1}+2\left(f_{1}-f_{2}\right) \alpha
$$

where $f=200 \mathrm{~mm}$ is the focal length of the lens. The radius (half width at half maximum intensity) of the diffraction limited focus at the detector plane is given by

$$
w=\frac{\lambda f_{2}}{2 f_{2} \mathrm{NA}_{1}+\left(f_{1}-f_{2}\right) \alpha} .
$$

The experimental results are displayed in Fig. 4.3. All widths are relative to the diffraction limit of the glass lens $\left(w_{0}=31 \mu \mathrm{m}\right)$. Without sample, the sharpest focus that was obtained by optimizing the wavefront has a width of 1.2 times this diffraction limit. The fact that the diffraction limit was not reached clearly demonstrates the limitations of the wavefront synthesizer. With a sample, however, these limitations do not degrade the sharpness of the focus. The width of the focus is exactly the diffraction limit that is predicted by Eq. (4.2). 


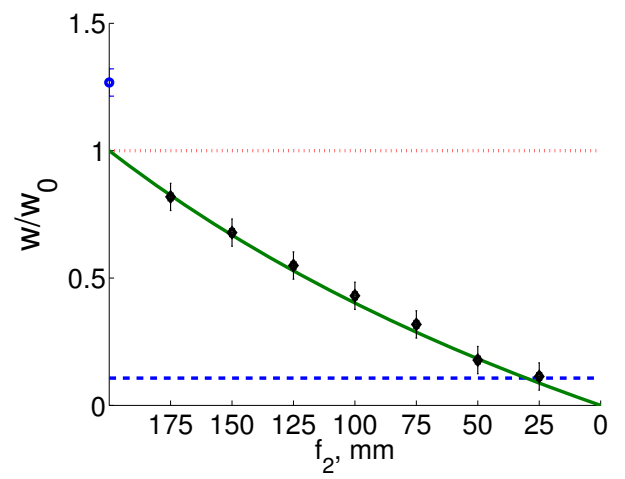

Figure 4.3: Relative focal width $w$ as function of sample-camera distance $f_{2}$. Diamonds, measured values with a sample present; solid curve, theoretical diffraction limit; circle (at $f_{2}=200 \mathrm{~mm}$ ), width of the focus after optimizing without sample; dashed line, size of a single pixel of the camera, detection limit. All widths are relative to the diffraction limit of the glass lens (dotted line, $\left.w_{0}=31 \mu \mathrm{m}\right)$.

\subsection{Measured relation between the focus and the speckle correlation function}

It appears that the focus of an opaque lens always has the same size as a typical speckle. In this section, we analyze this effect by comparing the measured profile of the focus to the measured speckle correlation function. The speckle correlation function $C$ is a measure for the shape of a typical speckle.[7] It is defined as

$$
C\left(x_{1}, y_{1} ; x_{2}, y_{2}\right) \equiv \frac{\left\langle I\left(x_{1}, y_{1}\right) I\left(x_{2}, y_{2}\right)\right\rangle}{\left\langle I\left(x_{1}, y_{1}\right)\right\rangle\left\langle I\left(x_{2}, y_{2}\right)\right\rangle}-1 \text {, }
$$

where the brackets denote spatial averaging over all speckles. We experimentally investigated the relation between the speckle correlation function and the generated focus. First, we used the geometry as shown in Fig. 4.1 with the sample as close to the camera as possible $(26 \mathrm{~mm})$. The resulting spot has a radius of $6.6 \pm 3 \mu \mathrm{m}$, which is hardly larger than the size of a single pixel on the camera $(6.45 \mu \mathrm{m})$. The speckle correlation function and the intensity profile of the spot overlap exactly, as can be seen in Fig. 4.4.

The same experiment was performed in a different geometry and with different samples. This time the incident light was focused using a microscope objective and the transmitted light was imaged using a second objective (see Chapter 3 ). For a relatively thick sample (egg shell, $430 \pm 30 \mu \mathrm{m}$ thick) we placed the detecting objective at a distance of $3.5 \pm 0.5 \mathrm{~mm}$ and found a very narrow focus of $1.1 \pm 0.2 \mu \mathrm{m}$ (see Fig. 4.5). In Fig. 4.6, the results for a thinner sample $\left(10.1 \pm 0.3 \mu \mathrm{m}\right.$ of rutile $\mathrm{TiO}_{2}$ pigment $)$ in the same configuration is shown. For this sample, we find a wider focus of $11 \pm 0.2 \mu \mathrm{m}$. In both cases, the profile of the focus overlaps with the speckle correlation function. 


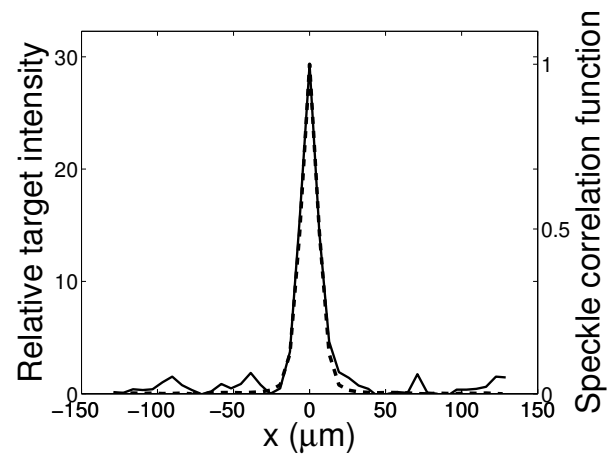

Figure 4.4: Intensity profile of the focus at $y=0$ (solid curve) and speckle correlation function (dashed curve) for an $8 \mu \mathrm{m}$ thick layer of airbrush paint creating a focus at $26 \mathrm{~mm}$ distance. The speckle correlation function was measured with a randomly generated incident wavefront.

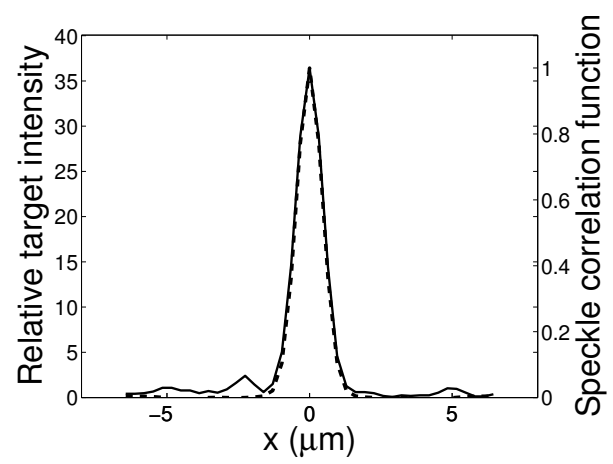

Figure 4.5: Intensity profile of the focus at $y=0$ (solid curve) and speckle correlation function (dashed curve) for an egg shell of $430 \pm 30 \mu \mathrm{m}$ thick creating a focus at $3.5 \pm 0.5 \mathrm{~mm}$ distance.

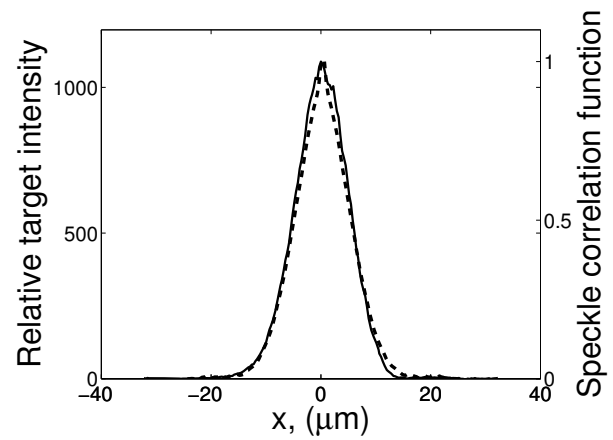

Figure 4.6: Intensity profile of the focus at $y=0$ (solid curve) and speckle correlation function (dashed curve) for a $10.1 \pm 0.3 \mu \mathrm{m}$ thick sample of white paint creating a focus at $3.5 \pm 0.5 \mathrm{~mm}$ distance. The central point of the correlation function was removed since it was affected by measurement noise in the camera image. 


\subsection{Continuous field theory for opaque lenses}

After optimizing to create a focus, a fraction of the field at the back of the sample forms a converging beam, while the rest of the field forms a random speckle. In Chapter 3, we introduced a matrix model to explain the intensity of the focus of an opaque lens. Although that model proved suitable for describing the intensity in the focus of an opaque lens, it cannot predict the shape of the focus. We now develop a continuous field formalism that explains both the intensity and the shape of the focus. This formalism also explains the similarity of the focus profile and the speckle correlation function that was observed in the experiment.

\subsubsection{Continuous field formalism}

Light propagates from the modulator to the sample, then it is transmitted by the sample, and finally it reaches the focal plane where the target detector is placed. We separate light propagation into two parts. The first part starts at the modulator and ends at the back of the sample. The second part is the propagation from the back of the sample to the focal plane.

We define coordinates in the focal plane as $\mathbf{r}_{b}\left(z_{b}=f_{2}\right.$ as defined in Fig. 4.1), on the back surface of the sample as $\mathbf{r}_{k}\left(z_{k}=0\right)$, and in the plane of the phase modulator as $\mathbf{r}_{a}$. The target for optimization is at point $\mathbf{r}_{\beta}$. The field at the back of the sample is given by

$$
E\left(\mathbf{r}_{k}\right)=\sum_{a}^{N} t_{a}\left(\mathbf{r}_{k}\right) E_{a},
$$

where the summation is over all segments of the phase modulator. We assume the field to be constant within a segment, so that we can write $E\left(\mathbf{r}_{a}\right)=E_{a}$. Just behind the modulator, light propagation is nearly perpendicular to the surface of the modulator. We can, therefore, use the Fresnel-Kirchhoff diffraction formula (see e.g. $[13,14]$ ) to find coefficient $t_{a}\left(\mathbf{r}_{k}\right)$

$$
t_{a}\left(\mathbf{r}_{k}\right)=\frac{i}{\lambda} \iint_{S_{a}} \mathrm{~d}^{2} \mathbf{r}_{a} G\left(\mathbf{r}_{k}, \mathbf{r}_{a}\right) .
$$

The integration is over the surface of a single segment of the light modulator $\left(S_{a}\right)$ and $G\left(\mathbf{r}_{k}, \mathbf{r}_{a}\right)$ is the unknown Green function for propagating from the modulator to the back of the sample. Light propagation from the back of the sample towards the focal plane is described by the free space Green function $g\left(\mathbf{r}_{b}-\mathbf{r}_{k}\right)$

$$
E\left(\mathbf{r}_{b}\right)=\iint \mathrm{d}^{2} \mathbf{r}_{k} g\left(\mathbf{r}_{b}-\mathbf{r}_{k}\right) \frac{i}{\lambda} E\left(\mathbf{r}_{k}\right) .
$$

Field propagation from a single segment $a$ to the focal plane is described by a transmission coefficient $t_{a}\left(\mathbf{r}_{b}\right)$

$$
t_{a}\left(\mathbf{r}_{b}\right) \equiv \iint \mathrm{d}^{2} \mathbf{r}_{k} g\left(\mathbf{r}_{b}-\mathbf{r}_{k}\right) \frac{i}{\lambda} t_{a}\left(\mathbf{r}_{k}\right) .
$$

The role of $t_{a}\left(\mathbf{r}_{b}\right)$ is similar to that of $t_{b a}$ in the matrix formalism. However, $t_{a}\left(\mathbf{r}_{b}\right)$ is a continuous function of the spatial coordinate and also gives the shape of the focus of the opaque lens. 


\subsubsection{Optimized field}

We consider the case where the incident field is shaped with a modulator that perfectly modulates phase and amplitude. In Chapter 8 , we will see that the results for imperfect modulation or phase only modulation are equal up to a constant prefactor. After optimizing for focusing at point $\mathbf{r}_{\beta}$, the field generated by the modulator is (see also Chapter 8)

$$
\widetilde{E}_{a}=E_{0} t_{a}^{*}\left(\mathbf{r}_{\beta}\right),
$$

where the tilde sign indicates the value of $E_{a}$ after optimization. $E_{0}$ is a constant that fixes the average intensity at the modulator to $I_{\text {in }}$, it is defined as

$$
\begin{aligned}
E_{0} & \equiv \frac{I_{\mathrm{in}}}{\sqrt{\frac{1}{N} I_{0}\left(\mathbf{r}_{\beta}\right)}}, \\
I_{0}\left(\mathbf{r}_{\beta}\right) & \equiv I_{\mathrm{in}} \sum_{a}^{N}\left|t_{a}\left(\mathbf{r}_{\beta}\right)\right|^{2} .
\end{aligned}
$$

A physical interpretation of $I_{0}\left(\mathbf{r}_{\beta}\right)$ is that it is the intensity in the focal plane averaged over all possible configurations of the light modulator that have an average intensity of $I_{\text {in }}$.

The field at the back of the sample after optimization is calculated by substituting Eq. (4.7) into Eq. (4.8) and then substituting the result in Eq. (4.4)

$$
\widetilde{E}\left(\mathbf{r}_{k}\right)=\sum_{a}^{N} t_{a}\left(\mathbf{r}_{k}\right)\left(E_{0} \iint \mathrm{d}^{2} \mathbf{r}_{k}^{\prime} g\left(\mathbf{r}_{\beta}-\mathbf{r}_{k}^{\prime}\right) \frac{i}{\lambda} t_{a}\left(\mathbf{r}_{k}^{\prime}\right)\right)^{*} .
$$

The term $t_{a}\left(\mathbf{r}_{k}\right) t_{a}^{*}\left(\mathbf{r}_{k}^{\prime}\right)$ fluctuates rapidly with the distance $\Delta \mathbf{r} \equiv \mathbf{r}_{k}^{\prime}-\mathbf{r}_{k}$. The ensemble average of this product is the field-field correlation function[15]

$$
\left\langle t_{a}\left(\mathbf{r}_{k}\right) t_{a}^{*}\left(\mathbf{r}_{k}^{\prime}\right)\right\rangle=\left\langle\left|t_{a}\left(\mathbf{r}_{k}\right)\right|^{2}\right\rangle \frac{\sin \left(k_{0}|\Delta \mathbf{r}|\right)}{k_{0}|\Delta \mathbf{r}|} \exp (-|\Delta \mathbf{r}| /(2 \ell)),
$$

where $k_{0} \equiv 2 \pi / \lambda$ and $\left|t_{a}\left(\mathbf{r}_{k}\right)\right|^{2}$ is the intensity transmission coefficient from segment $a$ to point $\mathbf{r}_{b}$. Its average value $\left\langle\left|t_{a}\left(\mathbf{r}_{k}\right)\right|^{2}\right\rangle$ depends on the geometry of the experiment and the thickness of the sample. Because the correlation function Eq. (4.12) is very sharp, we can take $g^{*}\left(\mathbf{r}_{\beta}-\mathbf{r}_{k}^{\prime}\right)$ out of the integral in Eq. (4.11). Integrating Eq. (4.12) over $\mathbf{r}_{k}^{\prime}$ gives.

$$
\iint \mathrm{d}^{2} \mathbf{r}_{k}^{\prime}\left\langle t_{a}\left(\mathbf{r}_{k}\right) t_{a}^{*}\left(\mathbf{r}_{k}^{\prime}\right)\right\rangle=\left\langle\left|t_{a}\left(\mathbf{r}_{k}\right)\right|^{2}\right\rangle 2 \pi \frac{1}{k_{0}^{2}+1 /(2 \ell)^{2}} \approx\left\langle\left|t_{a}\left(\mathbf{r}_{k}\right)\right|^{2}\right\rangle \frac{\lambda^{2}}{2 \pi},
$$

where we used that $4\left(k_{0} \ell\right)^{2} \gg 1$. This assumption is valid even for very strongly scattering samples. The area given by $\lambda^{2} /(2 \pi)$ corresponds to the size of a single scattering channel (see e.g. [16]). The average value of the optimized field at the back of the sample now reduces to

$$
\left\langle\widetilde{E}\left(\mathbf{r}_{k}\right)\right\rangle=g^{*}\left(\mathbf{r}_{\beta}-\mathbf{r}_{k}\right) C_{0} I_{0}\left(\mathbf{r}_{k}\right),
$$



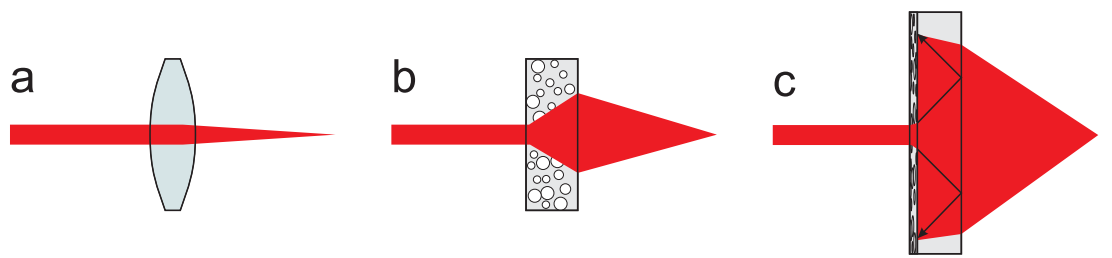

Figure 4.7: Better than diffraction limited focusing with an opaque lens. a) An ordinary lens at best focuses light to the diffraction limit given by the diameter of the incident beam. b) An opaque lens focuses light more sharply than the diffraction limit because diffusion in the lens increases the diameter of the transmitted converging beam. c) Opaque wafer lens made out of gallium phosphide. Reflection at the GaP-air interface fold back the optical paths and improve the focusing resolution even further.

where $C_{0} \equiv i \lambda E_{0} /\left(2 \pi I_{\text {in }}\right)$ and $I_{0}\left(\mathbf{r}_{k}\right) \equiv I_{\text {in }} \sum_{a}^{N}\left|t_{a}\left(\mathbf{r}_{k}\right)\right|^{2}$, just as in Eq. (4.10).

In the geometry as shown in Fig. 4.1, $I_{0}\left(\mathbf{r}_{k}\right)$ is constant in a disk with a diameter $D$ given by Eq. (4.1) and zero outside of the disk. In this case, Eq. (4.14) becomes

$$
\left\langle\widetilde{E}\left(\mathbf{r}_{k}\right)\right\rangle=g^{*}\left(\mathbf{r}_{\beta}-\mathbf{r}_{k}\right) C_{0} I_{0} \operatorname{circ}_{D}\left(\mathbf{r}_{k}\right)
$$

with

$$
\operatorname{circ}_{D}\left(\mathbf{r}_{k}\right) \equiv \begin{cases}0 & x_{k}^{2}+y_{k}^{2}>D^{2} / 4 \\ 1 & \text { otherwise. }\end{cases}
$$

Equation (4.15) tells us that, up to a constant proportionality factor, the average field at the back of the sample is the complex conjugate of the free space propagator. In other words, the transmitted field is the same as if the sample time-reversed a wave coming from the target focus. Hence, the transmitted light focuses on the target as well as is physically possible for a system with an aperture given by $\operatorname{circ}_{D}\left(\mathbf{r}_{k}\right)$.

In a different geometry, for example when the incident light is focused sharply on a thick sample, the intensity distribution $I_{0}\left(\mathbf{r}_{k}\right)$ will be a smooth function of the position. In such a geometry, an opaque lens differs from an ordinary lens. With an ordinary lens the amplitude profile at the back of the lens is equal to the amplitude profile of the incident illumination. For an opaque lens, however, the amplitude of the controlled field at the back of the sample is proportional to the intensity distribution at the back of the sample. In the following section we will see how to make use of this difference.

\subsubsection{Better than diffraction limited focusing}

In time-reversal microwave experiments it was observed that scattering improves the focusing resolution beyond the diffraction limit by 'folding' the propagation paths in a waveguide.[1] In this section, we show that a similar dramatic improvement of the focusing resolution can also be obtained in an optical system with a slab geometry.

With an ordinary lens, the intensity profile of the transmitted is the same as that of the incident light. For a given diameter of the incident beam, the lens at best focuses to the diffraction limit $\lambda f / D$ (see Fig. 4.7a). 
With an opaque lens, however, diffusion in the lens makes that the transmitted spot is larger than the incident beam (Fig. 4.7b). Therefore, an opaque lens focuses light more sharply than the best possible ordinary lens. The intensity distribution $I_{0}\left(\mathbf{r}_{k}\right)$ at the back of the sample depends on the thickness and mean free path of the sample, as well as on the extrapolation ratios that describe the boundary conditions. The thicker the scattering medium, the sharper the focus.

The benefit of a thick scattering medium was observed in the experiment. Under the same experimental conditions, the focus of a $430 \mu \mathrm{m}$ thick opaque lens (Fig. 4.5) was ten times narrower than the focus of a $10.1 \mu \mathrm{m}$ thick opaque lens (Fig. 4.6).

A proposed special example of an opaque lens is shown in Fig. 4.7c. A thin layer on one side of a gallium phosphide ( $\mathrm{GaP}, \mathrm{n}=3.3$ ) wafer is made porous. The porous gallium phosphide scatters the light into a $2 \pi$ solid angle. However, due to the high index contrast at the back of the wafer, most of the transmitted light is internally reflected back to the scattering layer, where it is scattered by the porous GaP for a second time. By folding the optical paths this way, the spot at the back of the sample increases dramatically, and the focusing resolution improves accordingly.

We now estimate the factor by which a GaP wafer lens improves the diffraction limit. The critical angle for total internal reflection at the GaP-air interface is $\theta_{c}=$ $17.6^{\circ}$. For a wafer with a thickness of $L$, the incident spot needs to have a diameter of at least $2 \tan \theta_{c} L$ for the reflected internally reflected light to overlap the original spot. We neglect the contribution of light that is reflected at an angle of over $45^{\circ}$ and we take into account only the first reflection at the GaP-air interface. Even with this pessimistic estimate, a $0.5 \mathrm{~mm}$ thick opaque a GaP wafer lens already expands a $0.32 \mathrm{~mm}$ beam to $4 \mathrm{~mm}$ before focusing it. These realistic numbers indicate that a $0.5 \mathrm{~mm}$ thick wafer can replace a $6 \times$ beam expander and a perfectly corrected microscope objective. In a way, this wafer is a miniature Cassegrain objective.

\subsubsection{Intensity profile of the focus}

We use the general result in Eq. (4.14) to calculate the intensity distribution in the focal plane. Using Eqs. (4.6) and (4.14) we find

$$
\widetilde{E}\left(\mathbf{r}_{b}\right)=\frac{i}{\lambda} \iint \mathrm{d}^{2} \mathbf{r}_{k} g\left(\mathbf{r}_{b}-\mathbf{r}_{k}\right) g^{*}\left(\mathbf{r}_{\beta}-\mathbf{r}_{k}\right) C_{0} I_{0}\left(\mathbf{r}_{k}\right) .
$$

In the paraxial limit (see e.g. [14]), we can approximate

$$
g\left(\mathbf{r}_{b}-\mathbf{r}_{k}\right) g^{*}\left(\mathbf{r}_{\beta}-\mathbf{r}_{k}\right) \approx \frac{e^{i \Phi}}{f_{2}^{2}} \exp \left(\frac{i k_{0}}{f_{2}}\left[x_{k}\left(x_{\beta}-x_{b}\right)+y_{k}\left(y_{\beta}-y_{b}\right)\right]\right),
$$

with $f_{2}$ the focal distance of the opaque lens. We isolated phase factor $\Phi \equiv x_{\beta}^{2}-x_{b}^{2}+$ $y_{\beta}^{2}-y_{b}^{2}$. We now have

$$
\left\langle\widetilde{E}\left(\mathbf{r}_{b}\right)\right\rangle=\frac{i e^{i \Phi}}{\lambda f_{2}^{2}} C_{0} \iint \mathrm{d}^{2} \mathbf{r}_{k} I_{0}\left(\mathbf{r}_{k}\right) \exp \left(\frac{i k_{0}}{f_{2}}\left[x_{k}\left(x_{\beta}-x_{b}\right)+y_{k}\left(y_{\beta}-y_{b}\right)\right]\right) .
$$


In Eq. (4.19) we recognize a 2-dimensional Fourier transform. We define the Fourier transformed intensity $U_{0}$ as

$$
U_{0}\left(\mathbf{r}_{b}\right) \equiv \iint \mathrm{d}^{2} \mathbf{r}_{k} I_{0}\left(\mathbf{r}_{k}\right) \exp \left(\frac{i k_{0}}{f_{2}}\left[x_{k}\left(x_{\beta}-x_{b}\right)+y_{k}\left(y_{\beta}-y_{b}\right)\right]\right) .
$$

To conform to literature (see e.g. [7]), we adopt the notation

$$
\mathscr{F}\left\{I_{0}\left(\mathbf{r}_{k}\right)\right\} \equiv U_{0}\left(\mathbf{r}_{b}\right) .
$$

Now, the intensity in the focal plane is given by expanding $C_{0}$

$$
\widetilde{I}\left(\mathbf{r}_{b}\right)=\frac{1}{\left(2 \pi f_{2}^{2}\right)^{2}} \frac{N}{I_{0}\left(\mathbf{r}_{\beta}\right)}\left|\mathscr{F}\left\{I_{0}\left(\mathbf{r}_{k}\right)\right\}\right|^{2},
$$

which confirms the observation made in Section 4.4.2 that the opaque lens perfectly focuses a field that is proportional to the intensity distribution at the back of the sample. The intensity exactly at the center of the focus equals

$$
\widetilde{I}\left(\mathbf{r}_{\beta}\right)=\left(\frac{P_{\text {tot }}}{2 \pi f_{2}^{2}}\right)^{2} \frac{N}{I_{0}\left(\mathbf{r}_{\beta}\right)}
$$

where $P_{\text {tot }} \equiv \iint \mathrm{d}^{2} \mathbf{r}_{k} I_{0}\left(\mathbf{r}_{k}\right)$ is the average total power that is transmitted through the sample. Before optimization, the power is distributed over $2 \pi$ solid angle. Therefore, $I_{0}\left(\mathbf{r}_{\beta}\right)=P_{\text {tot }} /\left(2 \pi f_{2}^{2}\right)$ and

$$
\widetilde{I}\left(\mathbf{r}_{\beta}\right)=N I_{0}\left(\mathbf{r}_{\beta}\right),
$$

which means that the intensity in the target has increased by exactly a factor $N$. For phase only modulation, the enhancement reduces to $1+(N-1) \pi / 4$ (see Chapter 8). The matrix model used in Chapter 3 predicts the same intensity enhancement. However, the new formalism clearly shows that the enhancement needs to be measured exactly at the maximum of the focus and not, for instance, averaged over the whole speckle. This experimentally important detail was not clear from the matrix model. Moreover, the new formalism predicts the exact shape of the focus.

\subsubsection{Connection with speckle correlation function}

When the sample is thicker than a transport mean free path, the field at the back surface of the sample is uncorrelated on length scales larger than the wavelength of light (also see Eq. (4.12)) and, equivalently, the transmitted light has no preferential direction. For such a sample, in the paraxial limit the correlation function (Eq. (4.3)) only depends on the coordinate difference $\Delta \mathbf{r}_{b} \equiv \mathbf{r}_{b}-\mathbf{r}_{b^{\prime}}$. The van Cittert-Zernike theorem tells us that, under these conditions, the correlation function is given by[7, $17,18]$

$$
C\left(\Delta x_{b}, \Delta y_{b}\right)=\frac{1}{P_{\text {tot }}^{2}}\left|\mathscr{F}\left\{\left\langle I\left(\mathbf{r}_{k}\right)\right\rangle\right\}\right|^{2},
$$

where $I\left(\mathbf{r}_{k}\right)$ is the diffuse intensity distribution at the back surface of the sample and $\mathscr{F}\left\{\left\langle I\left(\mathbf{r}_{k}\right)\right\rangle\right\}$ is defined as in Eq. (4.21). When the phase modulator is configured to 
generate a random field, $\left\langle I\left(\mathbf{r}_{k}\right)\right\rangle=\left\langle I_{0}\left(\mathbf{r}_{k}\right)\right\rangle$. In this case, it follows from Eq. (4.22) that,

$$
\widetilde{I}\left(x, y, f_{2}\right)=C(x, y) N \frac{P_{\text {tot }}}{2 \pi f_{2}^{2}} I_{0}\left(\mathbf{r}_{\beta}\right) .
$$

Equation 4.26 predicts that the intensity profile of the focus is exactly equal to the speckle correlation function (up to a constant prefactor). This identity is striking because the intensity in the focus is the square of an amplitude, whereas the correlation function is an product of intensities. Our continuous field model explains the results of Section 4.3, where this identity was observed experimentally in different geometries.

\subsection{Conclusion}

We observed experimentally that opaque lenses focus light to a diffraction limited spot. This experimental observation is explained by a continuous field model that was developed in Section 4.4. The model provides a simple method to predict the focusing resolution of an opaque lens for different geometries. For instance, a thin opaque lens that is illuminated with a flat-top profile focuses light to a diffraction limited spot. Theoretically, the focusing resolution can even be improved beyond the diffraction limit by making use of the diffusion in a thick opaque object. Extremely sharp focusing can be achieved with a GaP wafer lens where the optical paths are folded back by total internal reflection.

The shape of the focus is exactly the same as the correlation function of the speckle that is generated by the opaque lens. This experimentally important observation was explained with the continuous field model for opaque lenses.

An opaque lens focuses light to a diffraction limited spot or even sharper. Additionally, opaque lenses are extremely thin and extremely cheap compared to a microscope objective. Therefore, disposable opaque lenses might replace conventional microscope objectives, for example in applications that require tight focusing in a polluting or corroding environment, or when space is tight.

\section{Bibliography}

[1] A. Derode, A. Tourin, and M. Fink, Time reversal versus phase conjugation in a multiple scattering environment, Ultrasonics 40, 275 (2002).

[2] R. S. Longhurst, Geometrical and physical optics, 3rd ed. (Longman, London, 1973).

[3] E. Hecht, Optics (Addison Wesley Longman, New York, 1998).

[4] Nikon Instruments Inc., http://www.nikoninstruments.com/infocenter.php ? n=Coma as consulted on December 17th, 2007.

[5] R. K. Tyson, Principles of adaptive optics, 2nd ed. (Academic Press, New York, 1998).

[6] M. Fink, D. Cassereau, A. Derode, C. Prada, P. Roux, M. Tanter, J.-L. Thomas, and F. Wu, Time-reversed acoustics, Rep. Prog. Phys. 63, 1933 (1999).

[7] J. W. Goodman, Statistical optics (Wiley, New York, 2000).

[8] Waves and imaging through complex media, edited by P. Sebbah (Kluwer Academic, Dordrecht, Netherlands, 2001).

[9] R. Pappu, B. Recht, J. Taylor, and N. Gershenfeld, Physical one-way functions, Science 297, 2026 (2002). 


\section{Bibliography}

[10] G. Lerosey, J. de Rosny, A. Tourin, and M. Fink, Focusing beyond the diffraction limit with far-field time reversal, Science 315, 1120 (2007).

[11] E. Abbe, Beiträge zur Theorie des Mikroskops und der mikroskopischen Wahrnehmung, Archiv für Mikroskopische Anat Entwicklungsmech. 9, 413 (1873), as discussed in Ref. [2].

[12] A. E. Siegman, Lasers (University Science Books, Sausalito, USA, 1986).

[13] M. Born and E. Wolf, Principles of optics, 6th ed. (Pergamon Press, Oxford, United Kingdom, 1993).

[14] G. R. Fowles, Introduction to modern optics, 2nd ed. (Dover Publications, New York, 1989).

[15] R. Pnini and B. Shapiro, Fluctuations in transmission of waves through disordered slabs, Phys. Rev. B 39, 6986 (1989).

[16] J. F. de Boer, Optical fluctuations on the transmission and reflection of mesoscopic systems, Ph.D. thesis, University of Amsterdam, Amsterdam, 1995, available on http://cops.tnw.utwente.nl.

[17] P. H. van Cittert, Die wahrscheinliche Schwingungsverteilung in einer von einer Lichtquelle direkt oder mittels einer Linse beleuchteten Ebene, Physica 1, 201 (1934), as discussed in Ref. [7].

[18] F. Zernike, The concept of degree of coherence and its application to optical problems, Physica 5, 785 (1938), as discussed in Ref [7]. 


\title{
Chapter 5 \\ Demixing light paths inside disordered metamaterials
}

\begin{abstract}
We experimentally demonstrate the first method to focus light inside disordered photonic metamaterials. In such materials, scattering prevents light from forming a geometric focus. Instead of geometric optics, we used multi-path interference to make the scattering process itself concentrate light on a fluorescent nanoscale probe at the target position. Our method uses the fact that the disorder in a solid material is fixed in time. Therefore, even disordered light scattering is deterministic. Measurements of the probe's fluorescence provided the information needed to construct a specific linear combination of hundreds of incident waves, which interfere constructively at the probe.

[This chapter has been published as: I. M. Vellekoop, E. G. van Putten, A. Lagendijk, and A. P. Mosk, Opt. Express 16, 67-80 (2008)]
\end{abstract}

Photonic metamaterials are materials that gain their optical properties from their nanostructure, rather than from their chemical composition. Their sub-wavelength elements interact very strongly with the electromagnetic field and provide unprecedented control over the propagation of light $[1,2]$. Exciting perspectives of metamaterials include field enhancement in photonic crystal cavities[3], negative refractive index optics[4-6], and sub-wavelength imaging[7, 8]. Perhaps even more amazing is the use of metamaterials to cloak objects, making them completely invisible for a certain frequency of light. $[1,2]$ While these perspectives require near-perfectly manufactured metamaterials, intentionally disordered metamaterials also find applications such as sub-wavelength focusing[9] and enhanced non-linear conversion[10, 11]. Furthermore, disordered metamaterials are of fundamental interest because of their analogy to electrical conductors[12, 13], their quantum optical properties[14], and mesoscopic transport phenomena[15-17]. Recently it was shown experimentally that localization induced by disorder can confine and guide light.[18]

To direct light to a desired position in a metamaterial requires thorough knowledge of the propagation of light in these structures. Since metamaterials are strongly photonic, even weak manufacturing imperfections strongly influence propagation[1921], which makes it even harder to get light to the right place. For disordered metamaterials it is, even in theory, impossible to have a priori knowledge of light propagation. To treat the metamaterial as a homogeneous medium and pretend to focus somewhere inside (Fig. 5.1a) is an erroneous approach; in reality, the structure of the material prevents light from forming any focus at all.

We demonstrate experimentally that light can be delivered to a target inside a disordered photonic metamaterial, or in fact any strongly scattering solid, without having any a priori knowledge of the optical properties of the system. Our approach is based on the fact that the structure of the metamaterial mixes light entering through 

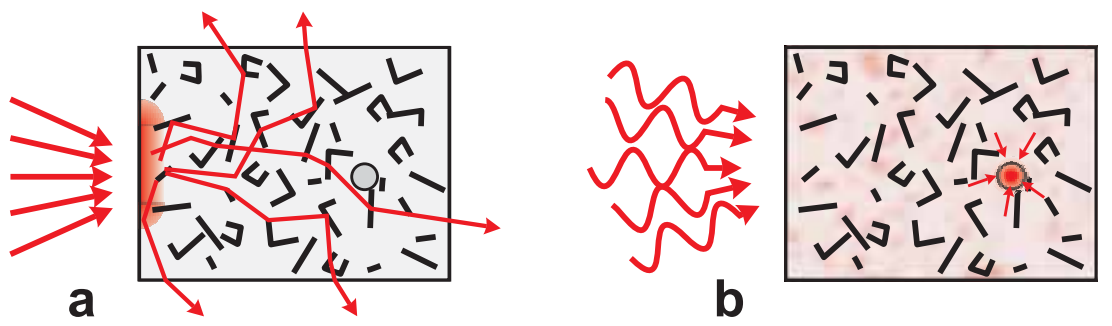

Figure 5.1: Principle of channel demixing. a) Conventional way of illuminating a metamaterial sample or device. In order to get light to the area inside the metamaterial indicated by the circle, one might try to focus light using an ordinary lens. However, the randomly oriented nanoparticles in the material mix the incident scattering channels in a complicated way so that light does not travel directly to the target. Incident rays will not converge to a geometrical focus. b) Our new way of illuminating a specific point inside a metamaterial. The wave nature of light is used as an advantage. When the phase delay for each of the incident scattering channels is set correctly, the channels demix inside the sample; multi-channel interference makes the light focus at the desired point.

different scattering channels (a scattering channel is an angular or spatial mode of the optical field[12]). The mixing process is complex and stochastic due to disorder. Even so, reciprocity dictates that there always exists a linear combination of incident channels that demix inside the metamaterial to focus at the target. In our experiment we find this unique configuration and focus light on a target that is deeply embedded in a disordered photonic structure (Fig. 5.1b).

It is well known that the inverse wave problem is ill posed. In other words, even in principle optical measurements cannot be used to reconstruct the three-dimensional structure of a photonic metamaterial. Likewise, optical reflection and transmission measurements cannot reveal the combination of channels that demix inside the material.

There exist different methods for focusing waves in the presence of scattering. In transparent media where geometrical optics applies, delivery of light can be achieved very effectively using a variety of adaptive optics wavefront correction techniques (see e.g. Ref. 22). For example, adaptive optics is commonly used to reduce astronomical seeing[23], to improve the resolution of ophthalmic imaging[24], or for femtosecond pulse shaping[25]. Unfortunately, these adaptive optics techniques break down in nanostructured media where geometrical optics is not applicable.

For ultrasound and microwaves time-reversal has proven highly effective[9, 26] in refocusing waves. Light waves can be refocused back to their source by phase conjugation[27], or they can be focused behind a scattering region by using feedback from an external detector[28]. All these methods require physical access to the target position, to place a transceiver, an extensive non-linear medium, or a detector. Inside a photonic metamaterial, with structures smaller than the wavelength of light, these methods cannot be used.

We have developed an approach that does allow focusing of light inside a metamaterial. Our method combines an advanced feedback and control system with a nanoscale fluorescent probe that is situated at the desired location. The power of the 
a

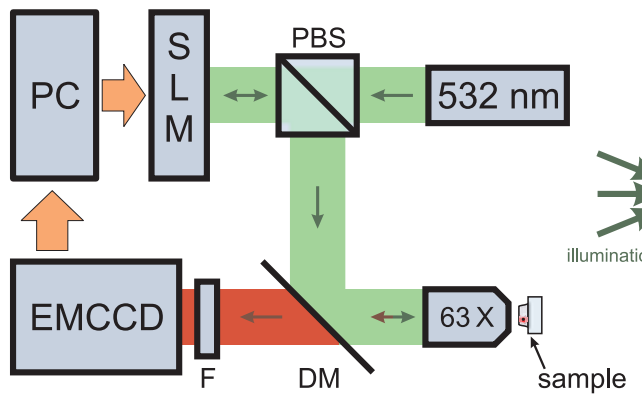

b

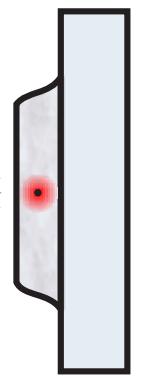

C

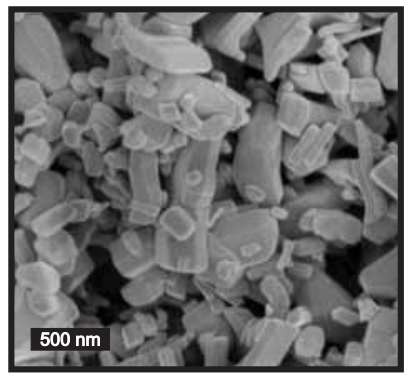

Figure 5.2: Experimental setup and samples. a) Simplified schematic of the experiment. A $532 \mathrm{~nm}$ laser is expanded and illuminates a spatial light modulator (SLM) that spatially modulates the phase of the reflected light. The SLM is imaged onto the back aperture of a $63 \mathrm{x}$ microscope objective that focuses the modulated light on a sample. Fluorescence light is collected by the same objective and imaged with an EMCCD camera. A computer drives the SLM and analyzes the EMCCD images. PBS, polarizing beam splitter; DM, dichroic mirror; F, fluorescence filter. Lenses were omitted from the schematic. b) Geometry of the samples. A low concentration of 300-nm diameter fluorescent spheres is dispersed in a zinc oxide pigment, deposited on a glass substrate. c) SEM image of a sample.

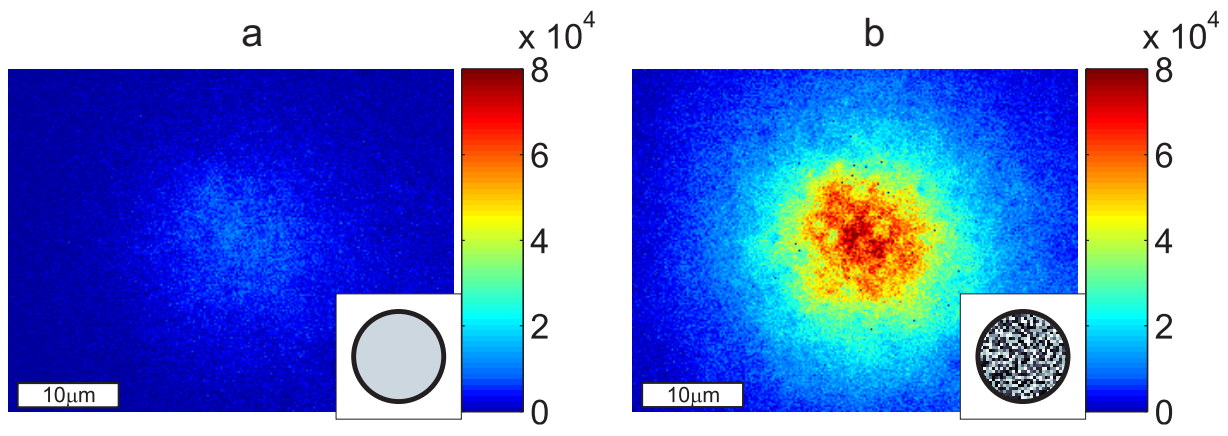

Figure 5.3: Experimental demonstration of channel demixing. a) Fluorescence image of a $300 \mathrm{~nm}$ sphere, embedded in $\mathrm{ZnO}$ pigment at a depth of $9.7 \mu \mathrm{m}$. Conventional illumination after a scan to find the position where the fluorescence is maximal; approximately 3 times the speckle average intensity. Inset, phase of the incident wavefront (plane wave). b) Our channel demixing method. Same sphere and sample position as in a, the phases of the incident channels were set to the measured optimal values. The resulting fluorescence intensity has increased by a factor of 22 with respect to the average intensity. Inset, phase of the incident wavefront. Inside the sample this seemingly random wavefront transforms into a focus. Intensities are in counts per second. 
emitted light is a measure for the intensity at the position of the probe. In our experiment (Fig. 5.2a), we used a liquid crystal spatial light modulator (SLM) that spatially modulates the phase of light coming from a green (532 nm) continuous wave laser. The pixels of the SLM are grouped into 640 square segments that are imaged onto the back aperture of a microscope objective. In this configuration, at the focal plane of the objective each segment corresponds to a solid angle encompassing approximately one mesoscopic scattering channel. For each of these incoming channels the phase can be set individually using the SLM. The microscope objective illuminates a sample that is mounted on a xyz-translation stage. The fluorescence light emitted by the probe inside the sample is collected by the same microscope objective and imaged with an electron multiplying charge coupled device (EMCCD) camera placed in a confocal reflection configuration. Our samples consist of a white layer of zinc oxide ( $\mathrm{ZnO}$ ) pigment on a glass substrate (Fig. 5.2b). The pigment grains have an average diameter of $200 \mathrm{~nm}$, resulting in a sub-wavelength disordered structure (Fig. 5.2c). The pigment layer is up to $32 \mu \mathrm{m}$ thick, and all samples are fully opaque. The transport mean free path was measured by fully analyzing transport of light through a series of samples (see Section 5.A). The samples were found to be strongly photonic with a mean free path of $0.7 \pm 0.2 \mu \mathrm{m}$, comparable to the wavelength of light. Fluorescent spheres with a diameter of $300 \pm 15 \mathrm{~nm}$ are sparsely dispersed through the sample.

Conventional focusing of light does not work in disordered photonic media. The absence of a ballistic focus was confirmed by performing an extensive 3-dimensional scan of the sample with respect to the microscope objective (see Section 5.A). The only change in the fluorescence emission was the result of the volume speckle field in the medium that is formed by scattered monochromatic light. As the sample moves, the weak fluorescence emission fluctuates randomly with the sample position. We consequently find that the maximal emission found by scanning the sample is only approximately 3 times the typical emission. In numerical simulations we find a similarly weak enhancement (Section 5.B). The fluorescence emission with the sample positioned at the brightest speckle is shown in Fig. 5.3a.

We now cycle the relative phase of a single incident channel between 0 and $2 \pi$ while keeping the phase of all other channels constant. The intensity at the probe is the result of interference between the modulated channel and the other channels, and therefore has a sinusoidal dependence on the phase. From the phase and the amplitude of the sinusoid we obtain the complex propagation amplitude from the modulated channel to the point inside the metamaterial. The complete set of propagation amplitudes contains all information that is needed to calculate the propagation of any incident wave towards the target. The optimal configuration of incident channels is found by taking the complex conjugate of the propagation amplitudes, so as to fully compensate for the mixing of the channels that will occur.

Figure 5.3b shows the strong increase in fluorescence emission that was achieved with channel demixing. By constructing the optimal configuration of incident channels, the intensity distribution inside the sample changed from random speckle to a pattern specially tailored for focusing light at the target. We find that the amplitudes for neighboring channels were uncorrelated, which is an indication that the channels were originally fully mixed. Light from different incident channels mixed while propagating in the medium and demixed again at the target position to form a focus. This 


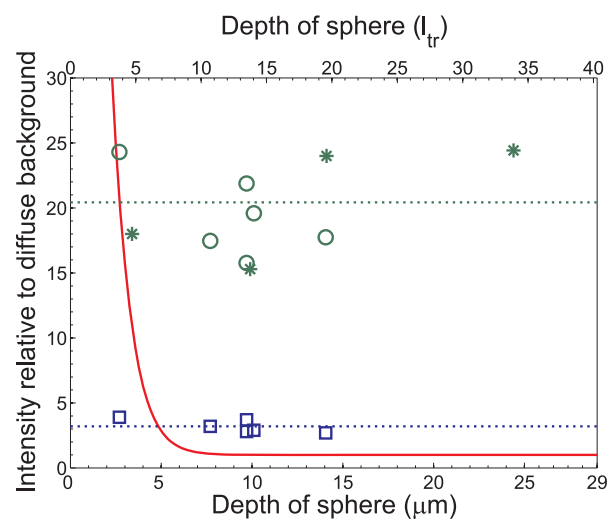

Figure 5.4: Measured intensity for targets at different depths inside a $29 \pm 3 \mu \mathrm{m}$ thick sample. Stars, results of our channel demixing technique, starting from a random sample position. An intensity was reached that is on average 20.4 times higher than the diffuse background (dotted line). Circles, results of our channel demixing technique starting on a bright speckle, demonstrating that starting from a bright spot is not advantageous. Squares, maximum intensity that was achieved by searching for the brightest speckle. An average intensity increase of only 3.2 was obtained (dotted line). Solid line, theoretical best case speckle average using adaptive optics, assuming that all geometrical aberrations are corrected perfectly. All measurements are normalized to the diffuse background.

focus was a factor of 22 more intense than the typical intensity and a factor of 6 more intense than the maximum that was achieved by searching for the brightest speckle.

The experiment was repeated for targets at different depths inside a $29 \pm 3 \mu \mathrm{m}$ thick sample. In Fig. 5.4 the target intensity that was reached with channel demixing is compared to the maximum intensity reached by translating the sample. Channel demixing increases the intensity at the target by a factor of on average 20.4, regardless of the depth of the target. It makes no difference whether the sample was meticulously placed on the brightest speckle first or whether the channel demixing procedure was started without a preceding scan of the sample position. For completeness, we also plot the calculated residual ballistic focusing component that can be expected when the sphere is placed exactly in the geometrical focus. For this calculation, we optimistically assumed that all geometric aberrations are perfectly corrected by an adaptive optics system[22]. As can be seen in Fig. 5.4, adaptive optics could be effective for targets that are only a few mean free paths deep. When the target lies deeper, scattering on the sub-wavelength structure of the material destroys even a perfectly corrected geometrical focus. Using channel demixing, however, we consequently find that the increase does not depend on the position of the target. Therefore, we conclude that scattering in no way limits the depth at which light can be delivered inside the metamaterial.

Theoretically, in the current experimental configuration, channel demixing has the possibility to achieve an intensity ratio that is approximately four times as high as the measured value of 20.4 (see Section 5.B). In practice, however, we are limited by photobleaching of the probe. We expect to achieve even higher intensity ratios as 
more stable probes, such as quantum dot assemblies[29], become available.

The measured set of complex propagation amplitudes characterizes propagation of light towards a sub-wavelength target inside the metamaterial. For this measurement no physical access to the target is required; it is not even required to know where the target probe exactly is. In both ordered and disordered metamaterials these amplitudes contain information about the structure and the optical quality of the metamaterial.[20, 30-32] This information, which was not experimentally accessible before, makes channel demixing a unique method for characterizing any metamaterial. Our experimental results call for the development of theoretical approaches that treat light propagation inside disordered photonic metamaterials.[33]

We have shown that light can be focused on a target inside a photonic metamaterial that completely scrambles the incident waves. Since the illumination is selective, channel demixing can be used to increase the contrast with a fluorescent background. A different application would be to selectively activate photochemical processes by concentrating light at a point inside a scattering material. Finally, the use of a fluorescent probe permits selectively focusing light on fluorescent areas, which is highly desirable in biological imaging, and may be a key technology for selectively illuminating individual cells in disordered biomaterials such as human skin tissue.

\section{A Experimental details}

\section{A.1 Apparatus}

The goal of the experiment is to illuminate a disordered medium in such a way that light focuses at a predefined target deep inside. The experimental setup consists of a spatial light modulator that is used to adjust the illumination, and a confocal fluorescence microscope that indirectly measures the intensity at the target. A detailed schematic of the setup is given in Fig. 5.5. Light from a continuous wave $532 \mathrm{~nm}$ laser (Coherent Compass M315-100) is expanded using a 30x beam expander. The expanded beam illuminates a Holoeye LC-R 2500 spatial light modulator (SLM) with a resolution of $1024 \times 768$ pixels. The pixels are grouped into square segments of 20 x 20 pixels. We have independent control over the phase and amplitude of each of the segments.[34] During this experiment, the amplitude of all segments is kept constant. The SLM is imaged onto the back aperture of a microscope objective using a 1:2 demagnifying telescope. In total, 640 segments fall within the aperture of the objective, which means that we control 640 degrees of freedom. The microscope objective (Zeiss infinity corrected 63x, NA=0.95 Achroplan air) focuses the light on the sample, thereby mapping the segments to channels in k-space. The sample is mounted on a XYZ-nanopositioning stage (Physik Instrumente P-611.3S NanoCube) to allow for a precise 3D-scan of the geometrical focus.

The returned fluorescence light is separated from the excitation light using a combination of a dichroic mirror (Semrock FF562-Di02-25x36) and a bandpass filter (Semrock FF01-593/40-25). An electron multiplying CCD camera (Andor Luca DL$658 \mathrm{M}$ ) images the fluorescence emission. A computer sums the intensity in the camera image to find the total power in the diffuse spot of fluorescence light. For each of the segments, measurements are performed at phase delays of $0,2 \pi / 5,4 \pi / 5,6 \pi / 5$, 


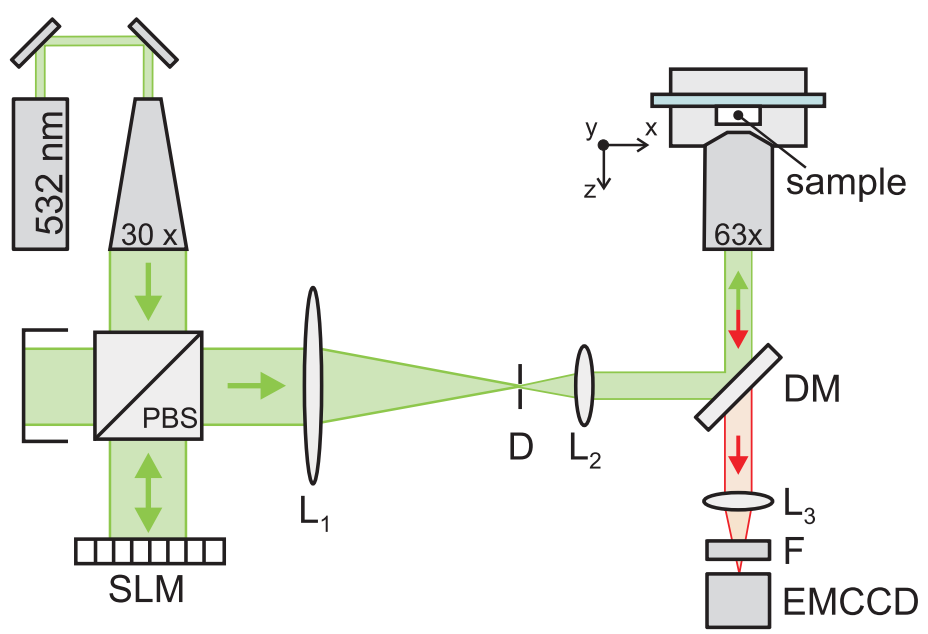

Figure 5.5: Experimental setup. The left part consists of a laser $(532 \mathrm{~nm})$ that is expanded by a 30x beam expander and modulated with a spatial light modulator (SLM). A 1:2 demagnifying telescope images the SLM on the back aperture of a $63 x$ microscope objective. The objective focuses the light on a sample that is mounted on a $X Y Z$ piezo positioning stage. A dichroic mirror (DM) and a bandpass filter (F) block the excitation light. The fluorescence emission is imaged on an electron multiplying CCD camera (EMCCD). D, iris diaphragm; PBS, polarizing beam splitter cube; $L_{1}, L_{2}, L_{3}$, lenses with a focal distance of $200 \mathrm{~mm}, 100$ $\mathrm{mm}$ and $150 \mathrm{~mm}$, respectively. Some folding mirrors and beam attenuation optics were omitted.

and $8 \pi / 5$ and a sinusoid is fitted to these measurements. Before advancing to the next segment, the phase delay is reset to 0 .

\section{A.2 Measurement sequence}

Channel demixing is achieved by measuring the propagation coefficients from the incident channels towards the embedded target. After all propagation coefficients are measured, it is known which linear combination of scattering channels focuses at the target. To quantify the effectiveness of this method, we measure the intensity at the probe position before and after the demixing procedure.

Since the field inside the medium is a completely random speckle pattern, the initial intensity is a random variable. A single measurement will not provide a reliable reference. Therefore, we measure the speckle averaged initial intensity $I_{0}$ by generating 500 different random combinations of incident channels and determining the average fluorescence intensity. This way, fluctuations caused by volume speckle are averaged out and a reliable measure for the initial intensity is found.

Next, the propagation constants for the different scattering channels are measured. This measurement takes approximately 18 minutes. During these measurements, the dye in the sphere photobleaches, which causes the response of the probe to decrease. Since we are interested in how much excitation light is focused on the target position, 


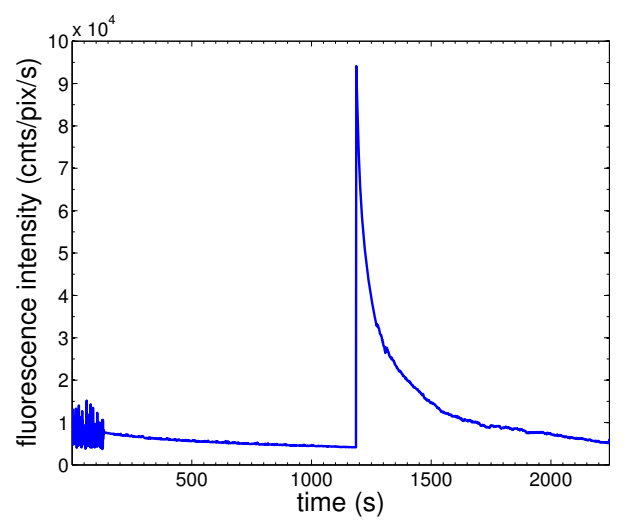

Figure 5.6: Evolution of the fluorescence intensity during the measurements. The measurement procedure has three phases. 0-130 s, reference measurement; $130-1187 \mathrm{~s}$, measurement of propagation coefficients; at $1187 \mathrm{~s}$, construction of optimal wavefront; $1187-2244 \mathrm{~s}$, fluorescence measurement with optimal wavefront.

we have to take into account the reduced response of the probe particle

$$
\eta_{\text {measured }}=\frac{I_{\mathrm{opt}}}{I_{0}} \frac{I_{1}}{I_{2}},
$$

where $I_{\mathrm{opt}}$ is the fluorescence intensity directly after constructing the optimal combination of scattering channels. $I_{1}$ is the fluorescence intensity at the start of this measurement step (directly after the reference measurement), and $I_{2}$ is the intensity at the end of the measurements (directly before the SLM is programmed with the optimal configuration). The ratio $I_{1} / I_{2}$ - which is between 1.0 and 1.8 when the sample is positioned at a typical position and between 1.5 and 3.9 when the sample is positioned at the brightest speckle - corrects for the fact that photobleaching decreases the sensitivity of the probe.

Figure 5.6 shows how the fluorescence intensity evolves during the measurement sequence. During the reference measurement, the intensity fluctuates rapidly. In the next step, the propagation constants are measured. Since we only cycle the phase of a single channel at a time, the illuminating wavefront is practically constant. During this step the fluorescence intensity decreases slowly due to photobleaching. After the measurements have been performed for all segments, the phase modulator is programmed with the optimal phases and the fluorescence intensity jumps to a higher value as the incident light now interferes constructively at the target. After this jump, the wavefront is kept constant. The reason that the fluorescence intensity decreases is that the higher incident intensity on the sphere causes the dye to bleach more rapidly.

\section{A.3 Sample preparation and characterization}

We demonstrated how interference of scattered light was used to focus light deep inside disordered metamaterials. Our samples are strongly scattering, that is, the mean free path for light is of the order of the wavelength. Inside the sample, fluorescent 


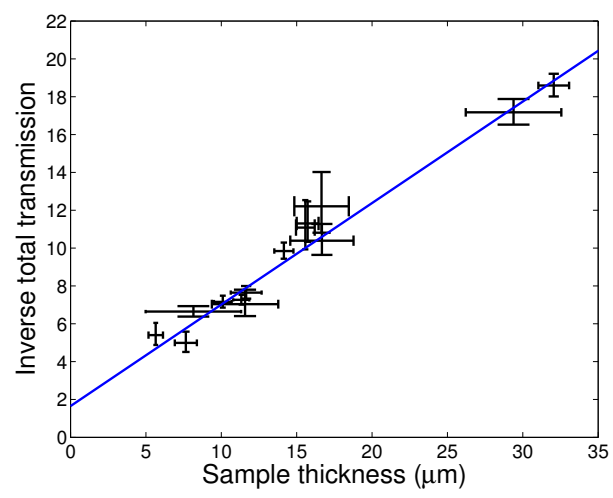

Figure 5.7: Inverse total transmission through layers of $\mathrm{ZnO}$ pigment. The solid line is a fit of Eq. (5.2) with $\ell=0.72 \mu \mathrm{m}, z_{e 1}=1.15 \mu \mathrm{m}$, and $z_{e 2}=1.24 \mu \mathrm{m}$. Error bars indicate the standard deviation of the measured thickness and total transmission at different positions on the sample.

probes mark the target positions. Typically, the probes are over 10 mean free paths deep, which makes it impossible to focus light on them using conventional means (Also see Section 5.B.4). In this section we address the fabrication and characterization of these samples.

\section{Sample preparation}

Our samples consist of a layer of Zinc Oxide pigments (Aldrich Zinc Oxide $<1 \mu \mathrm{m}$, $99.9 \%$ ). The average particle diameter is $200 \mathrm{~nm}$ (measured using scanning electron microscopy). The fluorescent probes are made from polystyrene and have a specified diameter of $300 \pm 15 \mathrm{~nm}$ (Duke Scientific red fluorescent nanospheres dyed with FireFly $\left.{ }^{\mathrm{TM}}\right)$. An aqueous suspension of pigment and spheres was sprayed on a standard microscope cover slide using a commercial airbrush (Harder \& Steenbeck Evolution). The thickness of the dried samples was measured with an optical microscope by making a scratch in the surface. The background fluorescence of the $\mathrm{ZnO}$ was shown to be negligible compared to the fluorescence of the dyed nanospheres.

\section{Measurement of the mean free path}

Transport of light in scattering media is quantified by the transport mean free path $\ell$. A method for obtaining $\ell$ is to measure the total (diffuse) transmission of light using an integrating sphere. $\ell$ then follows from the relation[35]

$$
\frac{1}{T}=\frac{L+z_{e 1}+z_{e 2}}{\ell+z_{e 1}}
$$

where $T$ is the total intensity transmission, $L$ is the sample thickness and $z_{e 1}, z_{e 2}$ are the extrapolation lengths that describe boundary effects on the front and back surfaces of the sample respectively. The extrapolation lengths follow from the ef-

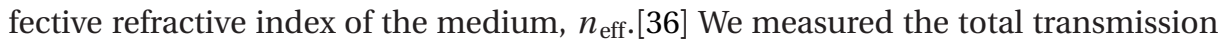


through a set of similar samples with varying thickness. The inverse total transmission for an ensemble of 14 samples is plotted in Fig. 5.7. The samples range in thickness from $6 \mu \mathrm{m}$ to $32 \mu \mathrm{m}$. As expected, the inverse total transmission increases linearly with sample thickness. We obtain a good linear fit for $\ell=0.7 \pm 0.2 \mu \mathrm{m}$ and $n_{\text {eff }}=1.4 \pm 0.1$. The extrapolation lengths can then be calculated from $n_{\text {eff }}$ and we find $z_{e 1}=1.15 \pm 0.2 \mu \mathrm{m}$ and $z_{e 2}=1.24 \pm 0.2 \mu \mathrm{m}$.

\section{Determination of the depth of the fluorescent probe}

For each experiment we select a different probe sphere at a different depth inside a sample. To determine the depth of the probe, we image the diffuse spot of emitted light and fit this data with diffusion theory. The accuracy of methods that rely on inverting the diffusion equation is limited to about one mean free path due to noise.[37]

The intensity inside a disordered medium with a source at depth $z_{0}$ follows from diffusion theory. We solved the diffusion equation for a slab of thickness $L$ using the Dirichlet boundary conditions $I\left(-z_{e 1}\right)=0$, and $I\left(L+z_{e 2}\right)=0[38,39]$. The extrapolation lengths $z_{e 1}$ and $z_{e 2}$ account for the reflections at the front and the back of the sample, respectively. A closed form solution is found using Fourier transformed traversal coordinates $\mathbf{q}_{\perp} \equiv\left(q_{\perp x}, q_{\perp y}\right)$.

$$
I_{d}\left(\mathbf{q}_{\perp}, z\right)= \begin{cases}J_{\operatorname{in}} \frac{\sinh \left(q_{\perp}\left[L_{e}-z-z_{e 1}\right]\right) \sinh \left(q_{\perp}\left[z_{0}+z_{e 1}\right]\right)}{D q_{\perp} \sinh \left(q_{\perp} L_{e}\right)} & z>z_{0} \\ J_{\operatorname{in}} \frac{\sinh \left(q_{\perp}\left[L_{e}-z_{0}-z_{e 1}\right]\right) \sinh \left(q_{\perp}\left[z+z_{e 1}\right]\right)}{D q_{\perp} \sinh \left(q_{\perp} L_{e}\right)} & z \leq z_{0}\end{cases}
$$

where $I_{d}$ is the diffuse energy density at depth $z, q_{\perp} \equiv \sqrt{q_{\perp x}^{2}+q_{\perp y}^{2}}$, and $J_{\text {in }}$ is the power of the source in Watts. $D$ is the diffusion constant and $L_{e} \equiv L+z_{e 1}+z_{e 2}$.

To obtain the depth of the source, we Fourier transform the microscope image of the fluorescence light and fit Eq. (5.3) with $\mathrm{z}=0$ using $z_{0}$ and an intensity prefactor as fit parameters.

\section{A.4 3-dimensional scan results}

Conventional focusing does not work in strongly scattering materials such as our samples. The absence of a ballistic focus was confirmed by performing an extensive 3 -dimensional scan of the sample with respect to the microscope objective. The fluorescence emission strongly fluctuates with the sample position. These fluctuations are the result of a volume speckle field in the medium that is formed by scattered monochromatic light. As the sample moves, the volume speckle fluctuates randomly. For each depth of the geometrical focus, we record the brightest speckle intensity. Figure 5.8 shows the results of a typical 3-D scan. The depth of the geometrical focus was estimated to be $n_{\text {eff }} z$, with $n_{\text {eff }}=1.4$ as measured, and $z$ the position of the translation stage. No sign of a ballistic focus was observed. In other words, it is not possible to focus light on the target using conventional means. 


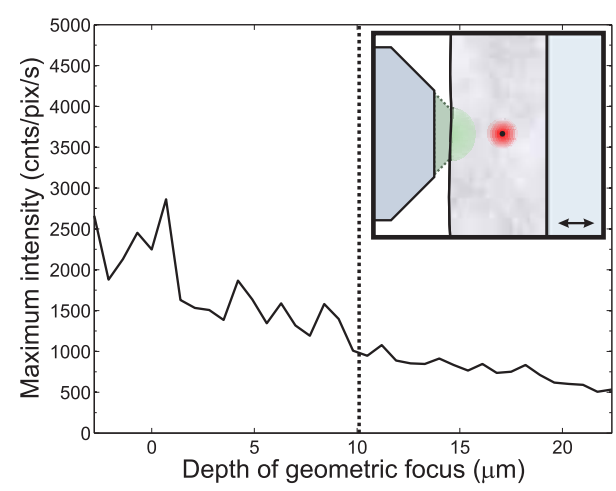

Figure 5.8: Determination of the absence of a geometrical focus. The sample is moved with respect to the objective (inset). For each depth, the sample is scanned in a plane parallel to the surface to find the maximum fluorescence intensity (solid line). In homogeneous media, there would be a sharp peak at the point where the fluorescent sphere is in the geometrical focus of the microscope objective (dotted line). In disordered photonic media, a geometric focus cannot be formed. The highest intensity is found when 'focusing' just below the sample surface.

\section{B Analysis of the channel demixing method}

The optical field $E$ at a point $\mathbf{r}_{b}$ inside the medium is given by

$$
E\left(\mathbf{r}_{b}\right)=\iiint \mathrm{d}^{3} \mathbf{r}_{a} G\left(\mathbf{r}_{b}, \mathbf{r}_{a}\right) S\left(\mathbf{r}_{a}\right),
$$

where $G$ is Green's function for propagating from the sources $S\left(\mathbf{r}_{a}\right)$ to point $\mathbf{r}_{b}$. In a disordered medium, $G$ is stochastic and, therefore, initially unknown. We treat each segment of the phase modulator as a sheet source with amplitude $A$ and phase $\phi$. Since the phase modulator is in good approximation illuminated homogeneously, all amplitudes $A$ are equal. By integrating over the surface area $S$ of each of the $N$ segments, Eq. (5.4) is discretized,

$$
\begin{aligned}
E\left(\mathbf{r}_{b}\right) & =\sum_{a}^{N} \iint_{S_{a}} \mathrm{~d}^{2} \mathbf{r}_{a} G\left(\mathbf{r}_{b}, \mathbf{r}_{a}\right) A e^{i \phi_{a}}, \\
& \equiv A \sum_{a}^{N} t_{b a} e^{i \phi_{a}} .
\end{aligned}
$$

When the phase of a single segment $a$ of the phase modulator is changed, the intensity at point $\mathbf{r}_{b}$ responds as

$$
I\left(\mathbf{r}_{b}\right) \equiv\left|E\left(\mathbf{r}_{b}\right)\right|^{2}=I_{0 b}+2 A \operatorname{Re}\left(E_{b \bar{a}}^{*} t_{b a} e^{i \phi_{a}}\right),
$$


with

$$
\begin{aligned}
I_{0 b} & \equiv\left|E_{b \bar{a}}\right|^{2}+A^{2}\left|t_{b a}\right|^{2}, \\
E_{b \bar{a}} & \equiv A \sum_{a^{\prime} \neq a}^{N} t_{b a^{\prime}} e^{i \phi_{a^{\prime}}} \approx E\left(\mathbf{r}_{b}\right) .
\end{aligned}
$$

Since the number of segments $N$ is large, $E_{b \bar{a}} \approx E\left(\mathbf{r}_{b}\right)$ and, therefore, equal for each of the segments. By repeating this procedure for all segments $a$, we measure the coefficients $t_{b a}$ up to an unknown common prefactor $E\left(\mathbf{r}_{b}\right)$.

Once all coefficients $t_{b a}$ are known, our channel demixing method maximizes $E\left(\mathbf{r}_{b}\right)$ by setting $\phi_{a}=-\arg \left(t_{b a}\right)$ for all segments. This configuration of phases gives the global maximum of Eq. (5.6),

$$
E_{\max }\left(\mathbf{r}_{b}\right)=A \sum_{a}^{N}\left|t_{b a}\right| .
$$

A comparison with Eq. (5.6) shows that now all amplitudes are summed with the same phase and that all incident channels will interfere constructively at the target.

\section{B.1 Maximum enhancement - scalar waves, simplified case}

The theoretical increase in intensity that can be achieved with channel demixing depends on the number of modulator segments $N$ and the statistical properties of coefficients $t_{b a}$. In disordered systems it is often allowed to assume that the coefficients are independently drawn from a single circular Gaussian distribution.[12, 13, 40-42] Under this assumption, the expected ratio between the original diffuse intensity and the maximally achievable intensity equals

$$
\eta=\frac{\pi}{4}(N-1)+1
$$

where $N$ can never exceed the number of mesoscopic scattering channels. The number of contributing scattering channels depends on the depth of the target. The deeper the target is embedded in the scattering material, the more channels will contribute to the mixing process. We estimate the number of channels that contribute to the target field by examining the size of the diffuse spot of the fluorescence light. The number of scattering channels in a spot with surface area $A$ is given by $[43,44] N_{\max }=2 \pi A / \lambda^{2}$. In Fig. $5.3 \mathrm{~b}$, the full width at half maximum of the diffuse spot is $15 \mu \mathrm{m}$, which leads to $A=177 \mu \mathrm{m}^{2}$ and $N_{\max }=3.9 \cdot 10^{3}$. In our experiment $N=640 \ll 3.9 \cdot 10^{3}$ and neighboring segments were uncorrelated.

\section{B.2 Maximum enhancement - finite size probe}

Ideally, the sphere that is used as a probe is only sensitive to the field of a single polarization at a single point. In reality, however, the fluorescent sphere probes the summed intensity of three polarizations in a finite volume. Therefore, it is as if we have multiple targets for which we try to increase the intensity simultaneously. As a 
result, the extra intensity is divided over all targets. The ratio between the original diffuse intensity and the maximally achievable intensity now equals

$$
\eta_{\mathrm{M}}=\frac{\pi}{4} \frac{N-1}{M}+1
$$

where $M$ is the number of targets. We determine $M$ by calculating how many orthogonal fields can exist in the volume of the probe sphere. Using Mie theory we find that for spheres of $300 \pm 15 \mathrm{~nm}$ the three electric dipole modes and the three magnetic dipole modes all give an equally strong contribution to the fluorescence power. The sphere is too small to support higher order (quadrupole) modes, so we estimate that the probe contains 6 optical modes. Using Eq. (5.12) with $M=6$ and $N=640$, we find an expected theoretical enhancement of 85. The experimental enhancement was approximately $25 \%$ of this value. We believe that the most important reason for not reaching the theoretical maximum enhancement is photobleaching. The channel demixing method favors the mode that initially is the brightest. Unfortunately, this target mode also photobleaches the fastest. Since we correct only for the average photo bleaching rate (see Section 5.A.2), the measured enhancement will systematically be lower than the actual enhancement.

\section{B.3 Maximum enhancement of speckle scan}

When the sample is moved, the speckle pattern in the medium fluctuates. As a result, the probe fluorescence will fluctuate too. Therefore, when a 3D-scan of the sample position is performed, at some points the intensity will be somewhat higher than the diffuse background. We used numerical simulations to calculate the expected maximum enhancement that this scanning technique can achieve. We assumed Rayleigh statistics for the speckle field and calculated the summed intensity in 6 independent optical modes at $N_{r}$ different positions. The simulation was repeated 1000 times and for each run the maximum speckle intensity was stored. The number of independent speckles, $N_{r}$, is limited by the diffuse intensity envelope of the light inside the material. We estimate that $N_{r}$ is between 1000 and 10000, depending on the depth of the probe. With these numbers, the simulations predict that the brightest speckle is 2.9 to 3.4 times as intense as the diffuse background.

\section{B.4 Diffuse and ballistic intensities inside the medium}

\section{Diffuse intensity}

When a slab of diffusive material is illuminated, the incident light can be described by a diffuse source at depth $z_{0}=\ell$ in the medium. [45] The maximum diffuse intensity at depth $z$ is found by inverse Fourier transforming Eq. (5.3) with $x=y=0$.

$$
I_{d}(x=0, y=0, z)=\frac{1}{2 \pi} \int_{0}^{\infty} \mathrm{d} q_{\perp} I_{d}\left(q_{\perp}, z\right) S\left(q_{\perp}\right) q_{\perp} .
$$

Where $S\left(q_{\perp}\right)$ accounts for the spatial extent of the source. We modelled the source as a Gaussian spot that encompasses 640 scattering channels; $S\left(q_{\perp}\right)=\exp \left(-q_{\perp}^{2} / 0.43\right)$. Eq. (5.13) was evaluated numerically. The diffuse intensity was used to normalize the theoretical curve for the perfect ballistic intensity in Fig. 5.4. 


\section{Perfect ballistic intensity}

We assume that all geometrical abberations are perfectly corrected and that all the incident light is directed towards a sphere in the geometrical focus. While travelling through the disordered medium, scattering and diffraction on sub-wavelength structures attenuate the incident beam according to the Lambert-Beer law,

$$
I_{b}=\frac{e^{-z / \ell_{s c}}}{A v}
$$

where $I_{b}$ is the ballistic energy density at the sphere, $A$ is the cross-section area of the sphere, $z$ is the depth of the sphere, $v$ is the velocity of light inside the medium and $\ell_{s c}$ is the scattering mean free path for light. The parameters $\ell_{s c}$ and $v$ have not been measured directly for our samples. We can, however, give an upper limit for the ballistic intensity by overestimating $\ell_{s c}=\ell$ and underestimating $v=3 D / \ell$. The ballistic intensity was normalized by the diffuse intensity and plotted in Fig. 5.4.

\section{Bibliography}

[1] U. Leonhardt, Optical conformal mapping, Science 312, 1777 (2006).

[2] J. B. Pendry, D. Schurig, and D. R. Smith, Controlling electromagnetic fields, Science 312, 1780 (2006).

[3] T. Yoshie, A. Scherer, J. Hendrickson, G. Khitrova, H. M. Gibbs, G. Rupper, C. Ell, O. B. Shchekin, and D. G. Deppe, Vacuum Rabi splitting with a single quantum dot in a photonic crystal nanocavity, Nature 432, 200 (2004).

[4] H. J. Lezec, J. A. Dionne, and H. A. Atwater, Negative refraction at visible frequencies, Science 316, 430 (2007).

[5] G. Dolling, M. Wegener, C. M. Soukoulis, and S. Linden, Negative-index metamaterial at $780 \mathrm{~nm}$ wavelength, Opt. Lett. 1, 53 (2007).

[6] V. M. Shalaev, Optical negative-index metamaterials, Nature Photonics 1, 41 (2007).

[7] V. G. Veselago, The electrodynamics of substances with simultaneously negative values of $\epsilon$ and $\mu$, Sov. Phys. Usp. 10, 509 (1968).

[8] Z. Liu, H. Lee, Y. Xiong, C. Sun, and X. Zhang, Far-field optical hyperlens magnifying subdiffraction-limited objects, Science 315, 1686 (2007).

[9] G. Lerosey, J. de Rosny, A. Tourin, and M. Fink, Focusing beyond the diffraction limit with far-field time reversal, Science 315, 1120 (2007).

[10] M. Baudrier-Raybaut, R. Haidar, P. Kupecek, P. Lemasson, and E. Rosencher, Random quasi-phase-matching in bulk polycrystalline isotropic nonlinear materials, Nature $\mathbf{4 3 2}$, 374 (2004).

[11] M. I. Stockman, D. J. Bergman, C. Anceau, S. Brasselet, and J. Zyss, Enhanced secondharmonic generation by metal surfaces with nanoscale roughness: Nanoscale dephasing, depolarization, and correlations, Phys. Rev. Lett. 92, 057402 (2004).

[12] C. W. J. Beenakker, Random-matrix theory of quantum transport, Rev. Mod. Phys. 69, 731 (1997).

[13] J. B. Pendry, A. MacKinnon, and P. J. Roberts, Universality classes and fluctuations in disordered systems, Proc. R. Soc. Lond. A 437, 67 (1992).

[14] P. Lodahl, A. P. Mosk, and A. Lagendijk, Spatial quantum correlations in multiple scattered light, Phys. Rev. Lett. 95, 173901 (2005).

[15] M. Störzer, P. Gross, C. M. Aegerter, and G. Maret, Observation of the critical regime near Anderson localization of light, Phys. Rev. Lett. 96, 063904 (2006). 
[16] S. Zhang, B. Hu, P. Sebbah, and A. Z. Genack, Speckle evolution of diffusive and localized waves, Phys. Rev. Lett. 99, 063902 (2007).

[17] P. Sebbah, B. Hu, A. Z. Genack, R. Pnini, and B. Shapiro, Spatial-field correlation: The building block of mesoscopic fluctuations, Phys. Rev. Lett. 88, 123901 (2002).

[18] T. Schwartz, G. Bartal, S. Fishman, and M. Segev, Transport and Anderson localization in disordered two-dimensional photonic lattices, Nature 446, 52 (2007).

[19] Y. A. Vlasov, V. N. Astratov, A. V. Baryshev, A. A. Kaplyanskii, O. Z. Karimov, and M. F. Limonov, Manifestation of intrinsic defects in optical properties of self-organized opal photonic crystals, Phys. Rev. E 61, 5784 (2000).

[20] S. Hughes, L. Ramunno, J. F. Young, and J. E. Sipe, Extrinsic optical scattering loss in photonic crystal waveguides: Role of fabrication disorder and photon group velocity, Phys. Rev. Lett. 94, 033903 (2005).

[21] A. F. Koenderink, A. Lagendijk, and W. L. Vos, Optical extinction due to intrinsic structural variations of photonic crystals, Phys. Rev. B 72, 153102 (2005).

[22] R. K. Tyson, Principles of adaptive optics, 2nd ed. (Academic Press, New York, 1998).

[23] Adaptive optics in astronomy, edited by F. Roddier (Cambridge University Press, Cambridge, 1997).

[24] Special issue: Advances in retinal imaging, J. Opt. Soc. Am. A 24, 1223 (2007).

[25] A. M. Weiner, Femtosecond pulse shaping using spatial light modulators, Rev. Sci. Instrum. 71, 1929 (2000).

[26] M. Fink, D. Cassereau, A. Derode, C. Prada, P. Roux, M. Tanter, J.-L. Thomas, and F. Wu, Time-reversed acoustics, Rep. Prog. Phys. 63, 1933 (1999).

[27] Optical phase conjugation, edited by R. A. Fisher (Academic Press, New York, 1983).

[28] I. M. Vellekoop and A. P. Mosk, Focusing coherent light through opaque strongly scattering media, Opt. Lett. 32, 2309 (2007), Chapter 3 of this thesis.

[29] M. Han, X. Gao, J. Z. Su, and S. Nie, Quantum-dot-tagged microbeads for multiplexed optical coding of biomolecules, Nature Biotech. 19, 631 (2001).

[30] S. John and R. Rangarajan, Optimal structures for classical wave localization: an alternative to the ioffe-regel criterion, Phys. Rev. B 38, 10101 (1988).

[31] N. M. Shapiro, M. Campillo, L. Stehly, and M. H. Ritzwoller, High-resolution surface-wave tomography from ambient seismic noise, Science 307, 1615 (2005).

[32] R. L. Weaver and O. I. Lobkis, Fluctuations in diffuse field-field correlations and the emergence of the Green's function in open systems, J. Acoust. Soc. Am. 117, 3432 (2005).

[33] We thank Carlo Beenakker and John Pendry for discussions on this promising field.

[34] E. G. van Putten, I. M. Vellekoop, and A. P. Mosk, Spatial amplitude and phase modulation using commercial twisted nematic LCDs, Appl. Opt. (2008), accepted. Preprint avaiable on arXiv: physics.optics/0711.4301. Also see Section 2.1 of this thesis.

[35] J. G. Rivas, R. Sprik, C. M. Soukoulis, K. Busch, and A. Lagendijk, Optical transmission through strong scattering and highly polydisperse media, Europhys. Lett. 48, 22 (1999).

[36] M. U. Vera and D. J. Durian, Angular distribution of diffusely transmitted light, Phys. Rev. E 53, 3215 (1996).

[37] A. S. McLean and J. B. Pendry, Beyond diffusion to diffraction, J. Mod. Opt. 42, 2495 (1995).

[38] H. S. Carslaw and J. C. Jaeger, Conduction of heat in solids, 2 ed. (University Press, 1959).

[39] S. Chandrasekhar, Radiative transfer (Dover Publications, Inc., New York, 1960).

[40] J. W. Goodman, Statistical optics (Wiley, New York, 2000).

[41] N. Garcia and A. Z. Genack, Crossover to strong intensity correlation for microwave radiation in random media, Phys. Rev. Lett. 63, 1678 (1989).

[42] A. D. Mirlin, R. Pnini, and B. Shapiro, Intensity distribution for waves in disordered media: Deviations from rayleigh statistics, Phys. Rev. E 57, R6285 (1998).

[43] B. A. van Tiggelen, A. Tip, and A. Lagendijk, Dwell times for light and electrons, J. Phys. A 


\section{8 | Bibliography}

26, 1731 (1993).

[44] J. F. de Boer, Optical fluctuations on the transmission and reflection of mesoscopic systems, Ph.D. thesis, University of Amsterdam, Amsterdam, 1995, available on http://cops.tnw.utwente.nl.

[45] E. Akkermans, P. E. Wolf, and R. Maynard, Coherent backscattering of light in disordered media: Analysis of the peak line shape, Phys. Rev. Lett. 56, 1471 (1986). 


\section{Chapter 6}

\section{Exploiting the potential of disorder in optical communication}

Scattering of light is a long recognized problem that causes losses in optical telecommunication, and decreases the resolution of imaging systems[1]. However, impressive experiments utilizing ultrasound and radio waves have shown that, for these waves, scattering and interference can be used as an advantage.[2] Disordered scattering is used for ultrafast radiographic communication[3] and for sub-wavelength focusing of microwaves[4].

Unfortunately, these revolutionary methods have so far not been available in the optical regime. The reason is that recording and playing back electrical fields at optical frequencies is close to impossible. Therefore, time-reversal - which is highly effective for sound and radio waves - is not a realistic option. In this chapter, we show that the potential of scattering can be exploited without time-reversal. A self-learning optical system uses scattering to improve its resolution beyond the limit imposed by diffraction at its aperture. Thanks to the increased resolution, multiple communication channels can coexist in the space that is normally needed to support one unscattered communication channel.

We first explain the principle of the experiment and present our results. Then, in Section 6.2, we give a more detailed description of the apparatus and the experimental procedure.

\subsection{Increasing the information density}

Figure 6.1a shows an optical system where a lens focuses monochromatic light on a camera. Due to the limited numerical aperture $(N A=1 / 200)$ of the system, the spot will at best have a diffraction limited width of $60 \mu \mathrm{m}$ (full width at half maximum). While aberrations in the optics can efficiently be removed using adaptive optics[5], the resolution of an adaptive optics system is still limited by its numerical aperture.

We choose three individual pixels of the camera to define three independent receivers. The spacing between the pixels is less than the diffraction limit of the lens. When the beam path is blocked by a disordered scattering medium (a $9 \mu \mathrm{m}$-thick layer of airbrushed white paint), the image on the camera changes dramatically. Instead of a focal spot, we now record a disordered speckle pattern that is the result of random interference. Although the scattered light does not focus, its features are much sharper than the size of the original diffraction limited spot because the scattering process increases the effective numerical aperture of the optical system.

To control the propagation of light through the disordered environment, we developed a learning algorithm that acquires an optical fingerprint (a spatial amplitude 

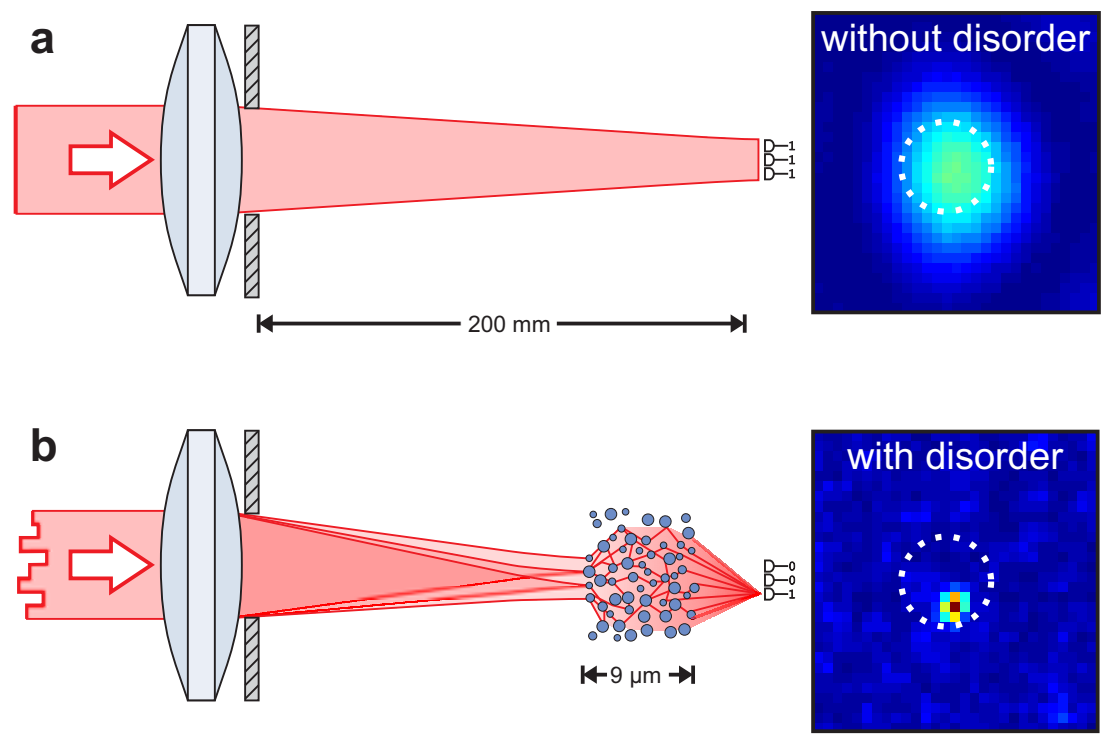

\section{C}
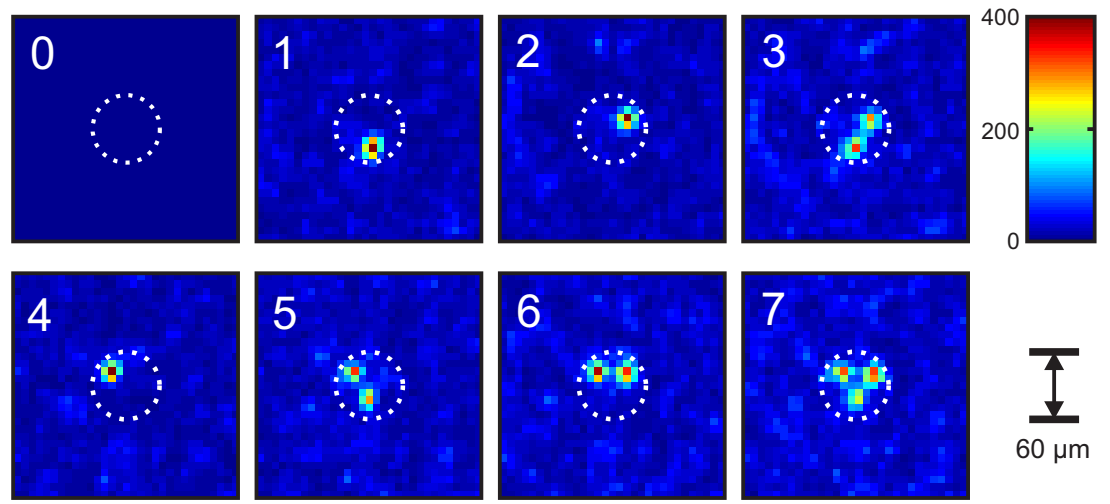

Figure 6.1: a) An optical system with a limited numerical aperture cannot focus light sharply, all three detectors receive the same signal. b) Scattering improves the diffraction limit. The incident light is spatially shaped to match a scattering fingerprint. Scattered light focuses sharply to a point. Now, each detector can be addressed individually. c) Measured transmitted intensity for eight different linear combinations of incident wavefronts. Any three-bit pattern can be generated in the area previously needed for one bit. 
and phase pattern) for each of the three receivers. The optical fingerprint uniquely describes propagation through the scattering medium towards the receiver. When the wavefront of the incident light matches this fingerprint, the scattered light converges to a focus at the receiver (Fig. 6.1b) and simultaneously the intensity on the other two receivers decreases.

We used a recently developed technique to spatially shape both phase and amplitude of a laser beam with a high accuracy.[6] This technique allowed us to construct any linear superpositions of fingerprints. Propagation through the scattering medium separates the superposition into separated foci on the receivers. All patterns of three bits can be generated, as is shown in Fig. 6.1c. A thin layer of white paint has increased the information density by a factor of three.

In conclusion, our method for matching optical fingerprints takes highly successful ideas from microwave technology and introduces them to the optical regime. Apart from improving optical communication, applications include sub-wavelength focusing with ordered[7] or disordered[4] metamaterials.

\subsection{Experimental details}

The setup consists of a low numerical aperture optical system and a wavefront synthesizer that spatially modulates light from a $632.8 \mathrm{~nm}$ Helium-Neon laser (see Figure 6.2). Wavefront modulation is achieved using a Holoeye LC-R 2500 spatial light modulator (SLM). The SLM is of the twisted nematic type and by itself does not allow independent control over the phase and amplitude of the reflected light. We developed a technique that combines four pixels into a macropixel of which both phase and amplitude can be controlled fully.[6]

Without scattering, the optical system is not able to create a sharp focus, regardless of the wavefront that the wavefront synthesizer produces. When we place a layer of strongly scattering paint in the optical path, light is scattered. The scattered light forms a random speckle pattern with sharp features. To focus light to a single point, we generate a wavefront that precisely matches the scattering matrix of the layer of paint. The scattered light now interferes constructively in a single point.

To construct the matching wavefront, or 'fingerprint', we use a method that is similar to the technique described in Chapter 3. One by one the phase of a segment of the phase modulator is cycled from 0 to $2 \pi$. Meanwhile, the intensity at the desired focal point is monitored with a camera. From the resulting interference signal we deduce the phase and amplitude of that segment's contribution to the target focus. This measurement is repeated for all segments. From these measurements, the unique[8] optical fingerprint that causes constructive interference in the target focus is constructed. In contrast to the method presented in Chapter 3, we also control the amplitude of the incident light. We set the amplitude of each of the segments to be proportional to the measured amplitude of that segment's contribution to the target focus. In this way the overlap of the incident field with the fingerprint of the medium is optimal. Segments that contribute little to the focus are effectively switched off so that the background intensity outside of the focus is reduced and contrast is improved. Theoretically, amplitude and phase modulation increases the contrast with the diffuse background by a factor of $4 / \pi$ with respect to phase only modulation. Moreover, thanks to the com- 


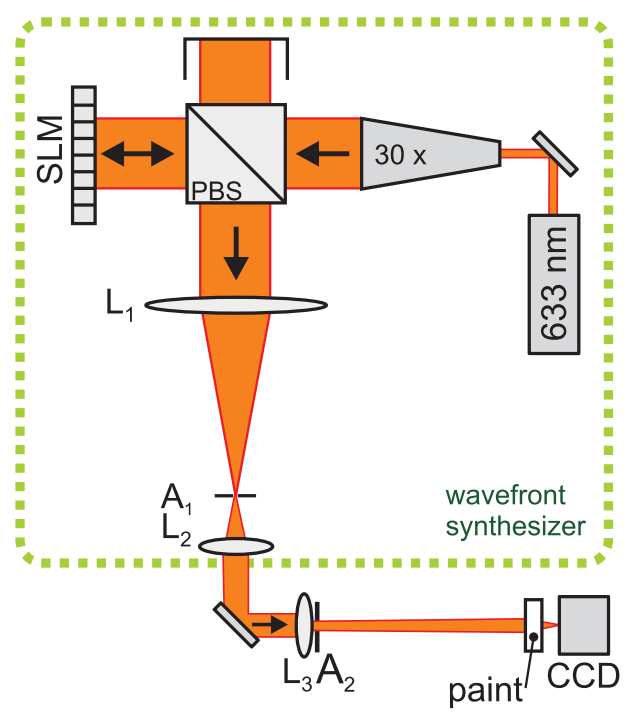

Figure 6.2: Schematic of the experimental setup. The first part of the setup consists of a wavefront synthesizer that modulates amplitude and phase of a $632.8 \mathrm{~nm}$ Helium-Neon laser. The laser beam is expanded by a $30 \mathrm{x}$ beam expander and modulated with a spatial light modulator (SLM). A 1:5 demagnifying telescope images the SLM on the surface of lens $L_{3}$. $A$ pinhole in the focal point of the telescope $\left(A_{1}\right)$ acts as a spatial filter, which allows the SLM to modulate both amplitude and phase (see text). The second part is the actual experiment, consisting of a camera (CCD) that is placed in the focal plane of lens $L_{3}$. The numerical aperture of the lens is lowered using a 2.1-mm diameter aperture $\left(A_{2}\right)$. At $26 \mathrm{~mm}$ from the camera a $9 \mu$ m-thick strongly scattering layer of white paint is placed. $A_{1}, A_{2}$, apertures; PBS, polarizing beam splitter cube; $L_{1}, L_{2}, L_{3}$, lenses with a focal distance of $250 \mathrm{~mm}$, $50 \mathrm{~mm}$ and $200 \mathrm{~mm}$, respectively. Some folding mirrors and beam attenuation optics were omitted.

bined amplitude and phase control, fingerprints for different foci can be superposed to generate any desired bit pattern.

\section{Bibliography}

[1] A. Ishimaru, Limitation on image resolution imposed by a random medium, Appl. Opt. 17, 348 (1978).

[2] S. H. Simon, A. L. Moustakas, M. Stoytchev, and H. Safar, Communication in a disordered world, Phys. Today 54, 38 (2001).

[3] G. J. Foschini, Layered space-time architecture for wireless communication in a fading environment when using multi-element antennas, Bell Labs Technical Journal 1, 41 (1996).

[4] G. Lerosey, J. de Rosny, A. Tourin, and M. Fink, Focusing beyond the diffraction limit with far-field time reversal, Science 315, 1120 (2007).

[5] R. K. Tyson, Principles of adaptive optics, 2nd ed. (Academic Press, New York, 1998).

[6] E. G. van Putten, I. M. Vellekoop, and A. P. Mosk, Spatial amplitude and phase modulation 
using commercial twisted nematic LCDs, Appl. Opt. (2008), accepted. Preprint avaiable on arXiv: physics.optics/0711.4301. Also see Section 2.1 of this thesis.

[7] C. Luo, S. G. Johnson, J. D. Joannopoulos, and J. B. Pendry, Subwavelength imaging in photonic crystals, Phys. Rev. B 68, 045115 (2003).

[8] R. Pappu, B. Recht, J. Taylor, and N. Gershenfeld, Physical one-way functions, Science 297, 2026 (2002). 
84 | Bibliography 


\title{
Chapter 7
}

\section{Phase control algorithms for focusing light through turbid media}

\begin{abstract}
Light propagation in materials with microscopic inhomogeneities is affected by scattering. In scattering materials, such as powders, disordered metamaterials or biological tissue, multiple scattering on sub-wavelength particles makes light diffuse. Recently, we showed that it is possible to construct a wavefront that focuses through a solid, strongly scattering object. The focusing wavefront uniquely matches a certain configuration of the particles in the medium. To focus light through a turbid liquid or living tissue, it is necessary to dynamically adjust the wavefront as the particles in the medium move. Here we present three algorithms for constructing a wavefront that focuses through a scattering medium. We analyze the dynamic behavior of these algorithms and compare their sensitivity to measurement noise. The algorithms are compared both experimentally and using numerical simulations. The results are in good agreement with an intuitive model, which may be used to develop dynamic diffusion compensators with applications in, for example, light delivery in human tissue.

[This chapter has been accepted for publication in Optics Communications.]
\end{abstract}

Materials such as paper, white paint or human tissue are non-transparent because of multiple scattering of light.[1-3] Light propagating in such materials is diffuse. Recently, we have shown that coherent light can be focused through diffusive media yielding a sharp, intense focus.[4] Starting with the situation where a scattering object (a layer of $\mathrm{TiO}_{2}$ pigment with a thickness of approximately 20 transport mean free paths) completely destroys the spatial coherence of the incident light (Fig. 7.1a, 7.1c), we controlled the incident wavefront to exactly match scattering in the sample. Afterwards, the transmitted light converged to a tight, high contrast focus (Fig. 7.1b, 7.1d). These matched wavefronts experience inverse diffusion, that is, they gain spatial coherence by travelling through a disordered medium.

For a given sample of scattering material, there is a unique incident wavefront that makes the object optimally focus light to a given point. Like a speckle pattern, this wavefront is disordered on the scale of the wavelength of light. This wavefront cannot be constructed from a small number of smooth base functions, which unfortunately renders the efficient algorithms used in adaptive optics (see e.g. [5]) ineffective. In Ref. 4, we presented an algorithm that finds the optimal wavefront when the sample is perfectly stationary and the noise level is negligible. To find applications in, for example, fluorescence excitation or photodynamic therapy, the wavefront has to be adjusted dynamically as the scatterers in the sample move. In this paper, we present two additional algorithms, that dynamically adjust the wavefront to follow changes 

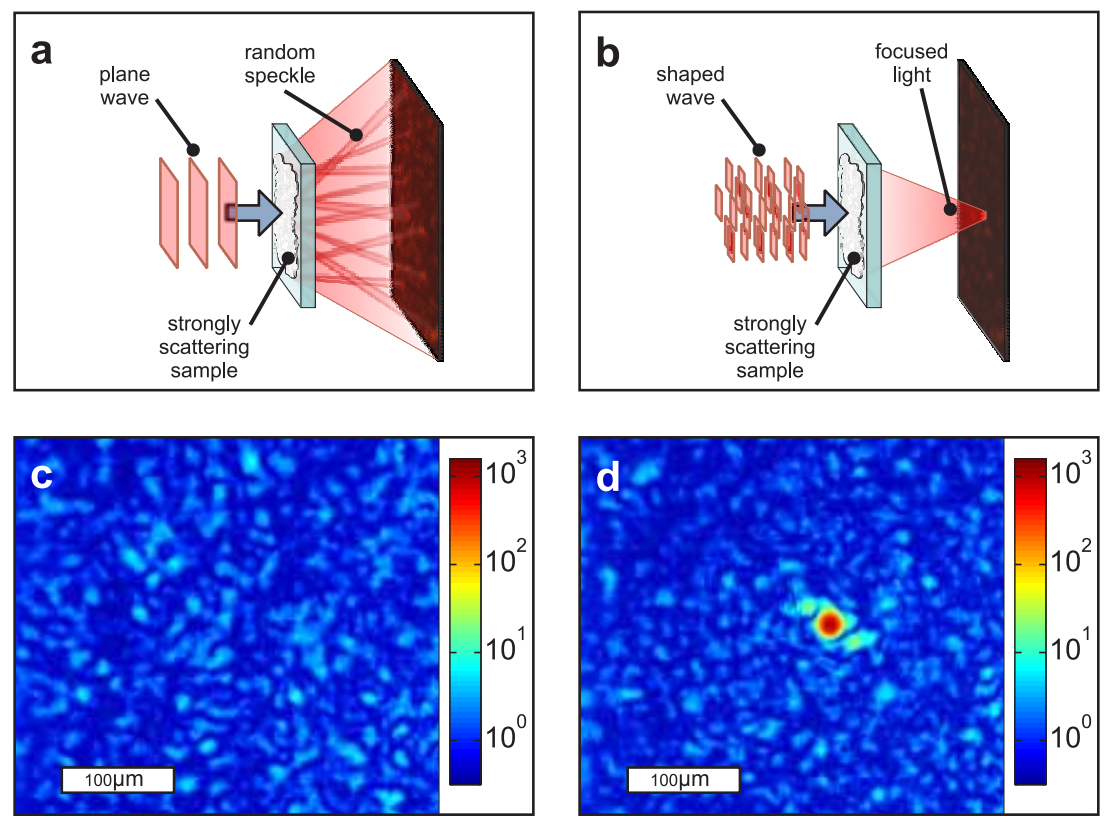

Figure 7.1: Principle and experimental results of inverse wave diffusion. a) A multiply scattering object destroys the spatial coherence of incident light. b) When the same object is illuminated with a specially constructed matching wavefront, the transmitted light focuses to a tight spot. c) Recorded intensity transmission of an unshaped wave through a $10 \mu \mathrm{m}$ thick layer of $\mathrm{TiO}_{2}$ pigment. d) Intensity transmission through the same sample with a shaped wavefront.

in the sample. The performance of the algorithms is in good agreement with numerical simulations and with an analytical model. We show that the new algorithms are superior to the original algorithm when the scatterers in the sample move or when the initial signal to noise ratio is poor.

Wave diffusion is a widely encountered physical phenomenon. The use of multiple scattered waves is the subject of intensive study in the fields of, for instance, ultrasound imaging[6-8], radio and microwave antennas[9, 10], seismography[11], submarine communication[12], and surface plasmons[13]. While the algorithms discussed in this paper were developed for spatial phase shaping of light, they can be used for any type of wave and apply to spatial phase shaping as well as to frequency domain phase shaping (also known as coherent control, see e.g. [14]) as the concepts are the same.

This article is organized as follows. First the key concepts of inverse diffusion are introduced and the three different algorithms are presented. Then the experimental apparatus is explained and the measured typical performance of the algorithms is compared. In the subsequent section, we will compare the experimental results with numerical simulations and analyze the data in terms of noise and stability of the scatterers. Finally, we will analytically explain the characteristic features of the different algorithms and discuss their sensitivity to noise. 


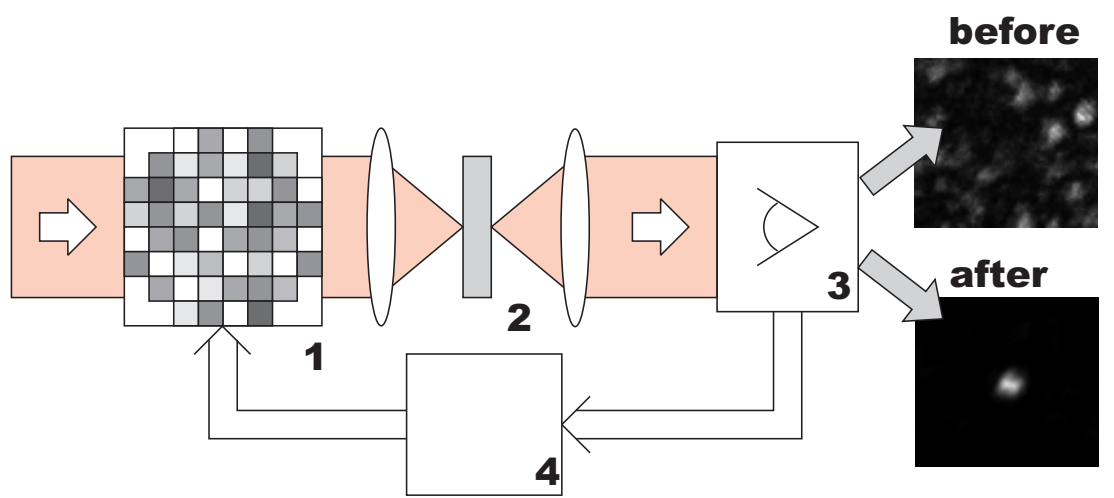

Figure 7.2: Feedback loop for achieving inverse diffusion. An incident monochromatic beam is shaped using a spatial light modulator (1) and projected on a non-transparent multiply scattering object (2). A detector (3) detects the amount of transmitted light that reaches the target area. A feedback algorithm (4) uses the signal from the detector to program the phase modulator. Before the algorithm is started, the transmitted light forms a random speckle pattern. The algorithm changes the incident wave to increase the intensity in the target area. After a few iterations, the transmitted light focuses on the target.

\subsection{Algorithms for inverse diffusion}

The key elements of an inverse diffusion setup are a multiply scattering sample, a spatial light modulator, an optimization algorithm and a detector, as shown in Fig. 7.2. The sample can be anything that scatters light without absorbing it. We will consider only samples that are thicker than approximately 6 transport mean free paths for light. Light transmitted through these samples is completely diffuse and the transmitted wavefront is completely scrambled, i.e., it has no correlation with the incident wavefront.[15] We assumed that the intensity is low so that non-linear effects are negligible.

The incident wavefront is constructed using a spatial phase modulator. The modulator consists of a 2D-array of pixels that are grouped into $N$ equally sized square segments. A computer sets the phase retardation for each of the segments individually to a value between 0 and $2 \pi$. The optimization algorithms program the phase modulator based on the detector output. Initially, each of the segments randomly contributes to the field at the detector. The algorithms find the unique configuration of retardations for which all contributions are in phase at the target. As follows from the triangle inequality, the magnitude of the field at the detector is at a global maximum for this optimal configuration. Since the sample completely scrambles the incident wavefront, all segments of the wavefront are scattered independently and the optimal wavefront will not be smooth.

Behind the sample is a detector that provides feedback for the algorithm. The detector defines the target area where the intensity is maximized. The field at the detector is the result of interference from scattered light originating from the different segments of incident wavefront. When the phase of one or more segments is changed, the target intensity responds sinusoidally. We sample the sine wave by taking 10 measurements. The process of capturing a single sine wave and possibly adjusting the 

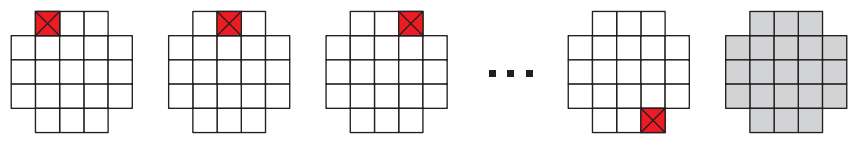

(a)
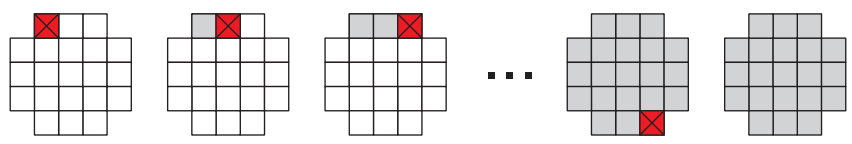

(b)
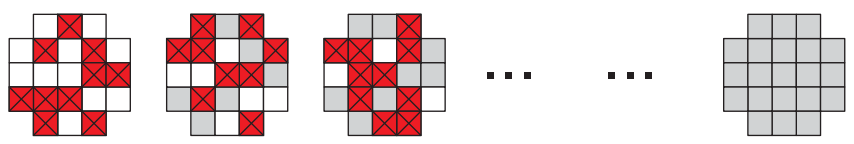

(c)

Figure 7.3: Principle used in the three different optimization algorithms. a) For the stepwise sequential algorithm, all segments are addressed sequentially (marked squares). After the optimal phase is measured for all segments, the modulator is updated to construct the optimal wavefront (light gray squares). b) The continuous sequential algorithm is equal to the first algorithm, except that the modulator is updated after each iteration. c) The partitioning algorithm randomly selects half of the segments and adjusts their overall phase. The modulator is updated after each measurement.

phase modulator accordingly is called an iteration.

The amount of control we have over the propagation of light in the disordered system is quantified by the signal enhancement. The enhancement $\eta$ is defined as

$$
\eta \equiv \frac{I_{N}}{\left\langle I_{0}\right\rangle},
$$

where $I_{N}$ is the intensity in the target after optimization and $\left\langle I_{0}\right\rangle$ is the ensemble averaged transmitted intensity before optimization. In a perfectly stable system, the enhancement is proportional to $N$ [4], meaning that the more individual segments are used to shape the incident wavefront, the more light is directed to the target. In practice, however, the enhancement is limited by the number of iterations that can be performed before the sample changes too much. We define the persistence time $T_{p}$ as the decay time of the field autocorrelate of the transmitted speckle, which is a measure for the temporal stability of the sample. The persistence time depends on the type of sample and on environmental conditions. Typical values of $T_{p}$ range from a few milliseconds in living tissue[16] to hours for solid samples in laboratory conditions. The other relevant timescale is the time required for performing a single iteration, $T_{i}$. In our experiments, we operate the phase modulator at just below $10 \mathrm{~Hz}$ and take ten measurements for each iteration; we have $T_{i} \approx 1.2 \mathrm{~s}$.

We will now present three algorithms we used to invert wave diffusion. The advantages and disadvantages of the algorithms are discussed briefly and will be analyzed in detail later.

\subsubsection{The stepwise sequential algorithm}

The stepwise sequential algorithm that was used in Ref. [4] is very straightforward. It is based on the fact that the field at the detector is a linear superposition of the contri- 
butions from all segments. This means that we can construct the optimal wavefront by optimizing each of the segments individually. The computer consecutively cycles the phase of each of the $N$ segments from 0 to $2 \pi$. The feedback signal is monitored and the phase for which the target intensity is maximal is stored. At this optimal phase, the contribution of this single segment is in phase with the background field (the mean contribution coming from all other segments). The phase retardation of the segment is reset to 0 before continuing with the next segment. This way, the background field remains unchanged. Only after all iterations are performed, the phase of each segment is set to this optimal value (see Fig. 7.3a). Now, all contributions have the same phase as the original background field.

In absence of measurement noise or temporal instability, algorithm 1 is guaranteed to find the global maximum in the lowest number of iterations possible. However, when $N T_{i} \gg T_{p}$, the speckle pattern decorrelates before all measurements are performed and the algorithm will not work. Therefore, it is important to adjust the number of segments to the persistence time.

\subsubsection{The continuous sequential algorithm}

The continuous sequential algorithm is very similar to the stepwise sequential algorithm except for the fact that the phase of each segment is set to its maximum value directly after each measurement (see Fig. 7.3b). This approach has two advantages. First of all, the algorithm runs continuously and dynamically follows changes in the sample's scattering behavior. Furthermore, the target signal starts to increase directly, which increases the signal to noise ratio of successive measurements. It is still necessary to adjust $N$ to the persistence time $T_{p}$.

\subsubsection{The partitioning algorithm}

The two sequential algorithms change the phase of one segment at a time. As one segment contributes only a small fraction of the wavefront, the signal to noise ratio is low and the initial convergence is slow. As an alternative, we propose a partitioning algorithm that simultaneously changes the phase of a whole group of segments (see Fig. 7.3c). The amplitude of the interference signal is maximal when the group contains half of the segments. At each iteration the segments are divided randomly over two equally sized partitions. Then, the target intensity is maximized by changing the phase of one partition with respect to the other. This process is repeated indefinitely, and the modulator is randomly repartitioned every time.

Since the phase of many segments is changed simultaneously, the initial increase in intensity will be fast and the feedback signal will be high. Therefore, this algorithm is expected to be less sensitive to noise and to recover from disturbances more rapidly.

\subsection{Experiment}

The different algorithms were tested experimentally using the setup shown in Fig. 7.4. In our case the scattering medium is a $10 \mu \mathrm{m}$ thick layer of rutile $\mathrm{TiO}_{2}$ pigment [17] 


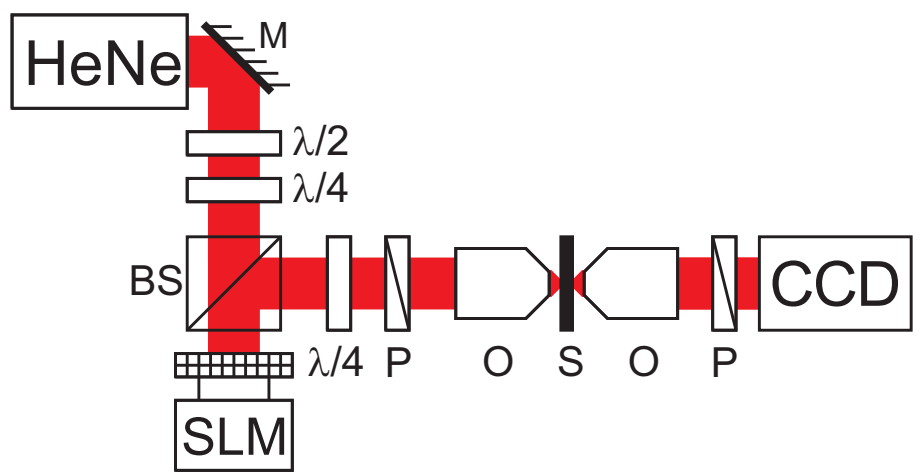

Figure 7.4: Experimental apparatus used for inverting diffusion. Light from a HeNe laser is spatially modulated by a liquid crystal spatial light modulator (SLM). Wave plates and a polarizer (P) are used to generate and select the polarization state for which the modulator works in phase-mostly mode. The shaped beam is demagnified by a factor of 3 (telescope not shown) and focused on the sample (S). A reference detector monitors the total intensity falling on the sample. A microscope objective, a polarizer and a CCD-camera are used to detect the intensity in the target focus, a few millimeters behind the sample. M, mirror; BS, non-polarizing $50 \%$ beam splitter.

with a mean free path of $0.55 \pm 0.1 \mu \mathrm{m}$, determined by measuring the total transmission at a wavelength of $632.8 \mathrm{~nm}$. This sample is illuminated by a $632.8 \mathrm{~nm} \mathrm{HeNe}$ laser. The laser beam is expanded and spatially modulated by a Holoeye LC-R 2500 liquid crystal light modulator (LCD). The modulator is a reflective twisted nematic liquid crystal on silicon (TN-LCoS) device. Such devices are designed for intensity modulation. However, by choosing an appropriate combination of the incident polarization and the analyzed polarization, the LCD can be used as a phase modulator with a minimal residual intensity modulation[18]. We characterized the LCD using the method described in [19] and select an optimal combination of elliptical polarizations to achieve a phase-mostly modulation mode with $2 \pi$ phase modulation and a maximum of $21 \%$ residual intensity modulation. The shaped beam is focused on the sample using a $63 x$ objective with a numerical aperture (NA) of 0.85 . A 20x objective $(\mathrm{NA}=0.5)$ images a point that is approximately $3.5 \mathrm{~mm}$ behind the sample onto a 12-bit CCD camera (Allied Vision Technologies Dolphin F-145B). This point is the target area where we want the light to focus. A computer integrates the intensity in a circular area with a radius of 20 pixels (corresponding to $129 \mu \mathrm{m}$ in the focal plane of the objective). This target area is smaller than a typical speckle spot. Using this signal as feedback, the computer programs the phase modulator using one of the algorithms described above.

We first run the three different algorithms with $N=52$. Since $T_{p} / T_{i} \gg 52$, we do not expect to see decoherence effects. In total, 208 iterations were performed, which means that the sequential algorithms ran four times consecutively. The results of the optimization procedures is shown in Fig. 7.5a. Although the three algorithms reach the same final enhancement of intensity, there are significant differences between the algorithms. The enhancement for the stepwise sequential algorithm increases 

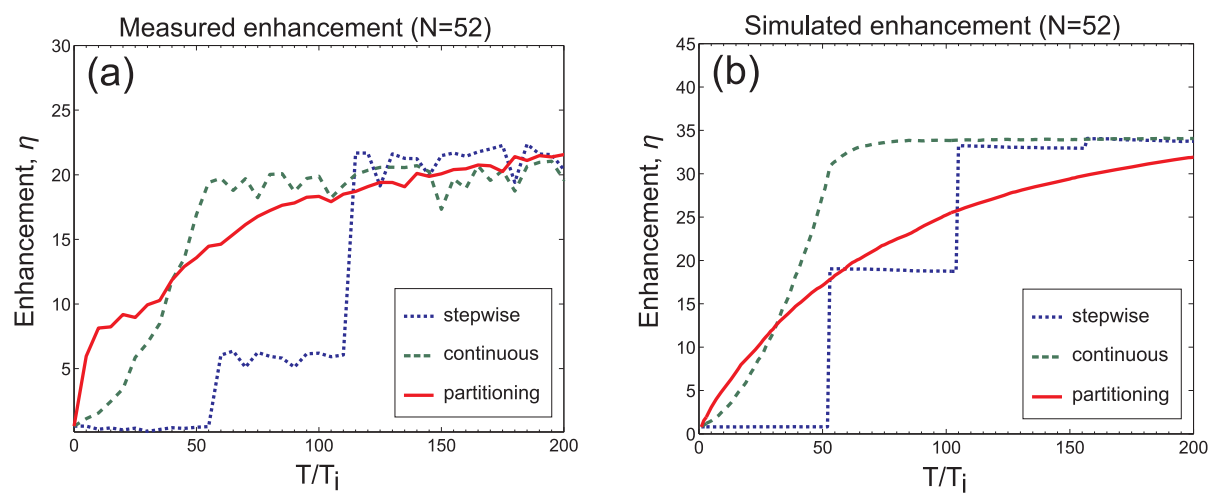

Figure 7.5: a) Typical runs of the stepwise sequential algorithm (dotted curve), the continuous sequential algorithm (dashed curve) and the partitioning algorithm (solid curve). All algorithms were run with $N=52$. The sequential algorithms were repeated four times. b) Simulation results for $N=52$ averaged over 64 runs. The simulation captures the main features of the three algorithms, but predicts a higher maximum enhancement.
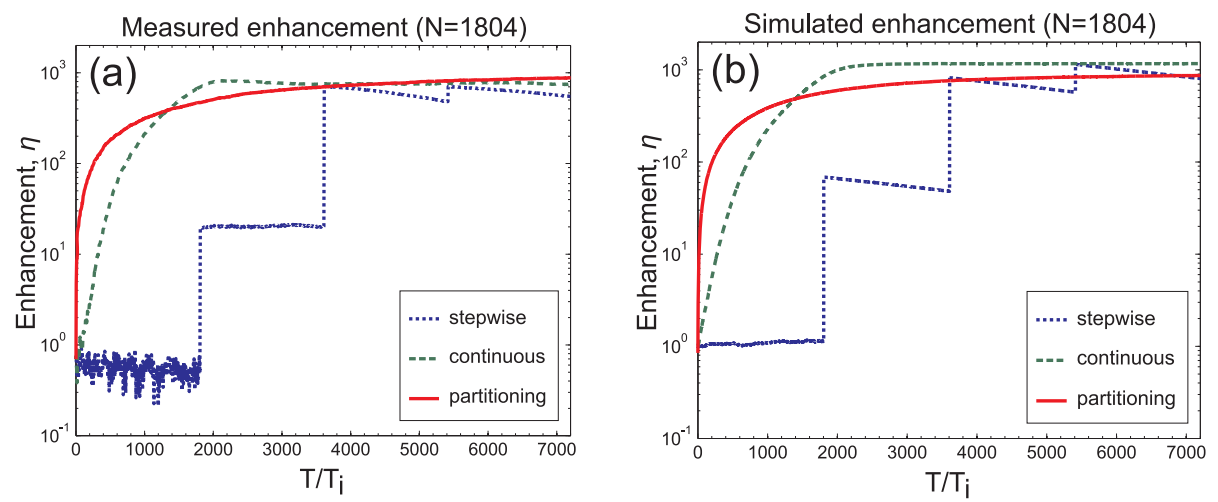

Figure 7.6: a) Typical runs of the stepwise sequential algorithm (dotted curve), the continuous sequential algorithm (dashed curve) and the partitioning algorithm (solid curve). All algorithms were run with $N=1804$. The enhancement are plotted on a logarithmic scale. b) Simulation results for $N=1804$ averaged over 64 runs.

in discrete steps because the phase modulator is only reprogrammed every $N$ iterations. During the first $N$ iterations, the target signal is low and the algorithm suffers from noise. The saturation enhancement is reached after the second update (after $2 N$ iterations). The continuous sequential algorithm and the partitioning algorithm both start updating the wavefront immediately and, therefore, have a higher initial increase of the signal. The continuous sequential algorithm is the first algorithm to reach the saturation enhancement (after $N$ iterations). The partitioning algorithm has the fastest initial increase in the target signal. It is, however, the last algorithm to reach the saturation enhancement since the final convergence is very slow.

When the number of segments in the wavefront is increased, we expect to find a 
higher target intensity. Figure 7.6a shows the experimental results for $N=1804$ on a logarithmic scale. The final intensity enhancement is approximately 40 times higher than in Fig. 7.5a. A further difference is that in this situation the effects of decoherence are no longer negligible. This effect is most clearly visible with the stepwise sequential algorithm. The phase modulator is updated after each $N$ iterations and between the updates the intensity decays exponentially with a $1 / e$ decay of about $T_{p} / T_{i}=5000$ iterations.

The convergence behavior of the three algorithms is similar to the experiment that is shown in Fig. 7.5. The partitioning algorithm clearly causes a higher signal enhancement during the first 1000 iterations. The initial increase in the enhancement is linear with a slope of 0.37 . Initially, this linear increase is far superior to the quadratic increase obtained with the continuous sequential algorithm.

We conclude that both new algorithms are valuable improvements over the original stepwise sequential algorithm. These algorithms are far less sensitive to noise and the target signal is kept at a constant value even in the presence of decoherence. The partitioning algorithm has the fastest initial increase and therefore will recover from disturbances most rapidly.

\subsection{Simulations}

In order to obtain a better understanding of the effects of noise and fluctuations on the performance of the different algorithms we perform numerical simulations. The disordered medium is represented by a transmission matrix with elements drawn from a circular Gaussian distribution (more details on the matrix representation can be found below). Decoherence is modelled by adding a small perturbation to the transmission matrix after every measurement. Finally, measurement noise is included by adding a random value to the simulated detector signal.

Figures 7.5b and 7.6b show the simulated enhancement for a system with $T_{p} / T_{i}=$ 5000. Every iteration, ten measurements are performed for phase delays between 0 and $2 \pi$. To each of these measurements, Gaussian noise with a standard deviation of $0.3 I_{0}$ was added. The magnitude of the noise is comparable to experimental observations. The three different algorithms were run with $N=52$ and $N=1804$ to simulate the experiments shown in Fig. 7.5a and Fig. 7.6a.

The simulations are in good qualitative agreement with the experimental data. The result for the stepwise sequential algorithm shows that the effects of noise and decoherence are simulated realistically. Furthermore, the initial signal increase and the long time convergence behavior correspond to the measured results. The only significant difference is the $20 \%$ to $50 \%$ higher enhancement reached in the simulations. A possible explanation for this difference is the residual amplitude cross-modulation in our phase modulator. Due to this cross-modulation, the optimal wavefront cannot be generated exactly. Furthermore, the amplitude modulation decreases the accuracy of the measurement of the optimal phase. The partitioning algorithm is less sensitive to this last effect since the cross-modulation is averaged over many segments with different phases. Since the simulations capture the overall behavior of the algorithms very well, we can use them to extrapolate to situations with a lot of noise and strong decoherence or, on the other hand, to perfectly stable systems. 


\subsection{Analytical expressions for the enhancement}

In this section, we analyze the performance of the algorithms with analytical theory and compare these results to the simulations. We describe scattering in the sample with the transmission matrix elements, $t_{m n}$. This matrix couples the fields of the incident light and the transmitted light.

$$
E_{m}=\sum_{n}^{N} t_{m n} A_{n} e^{i \phi_{n}},
$$

where the $\phi_{n}$ is the phase of the $n$th segment of the phase modulator. Assuming that the modulator is illuminated homogeneously, all incoming channels carry the same intensity. We write $A_{n}=1 / \sqrt{N}$ to normalize the total incident intensity. Elements $E_{1}, E_{2}, \ldots$ correspond to single scattering channels of the transmitted light. Since we are interested in focusing light to a single spot, we need to consider only a single transmission channel, $E_{m}$. The intensity transmitted into channel $m$ is given by

$$
\left|E_{m}\right|^{2}=\frac{1}{N}\left|\sum_{n}^{N} t_{m n} e^{i \phi_{n}}\right|^{2} .
$$

Regardless of the values of the elements $t_{m n}$ of the transmission matrix, the intensity $\left|E_{m}\right|^{2}$ has its global maximum when the phase modulator exactly compensates the phase retardation in the sample for each segment, i.e. $\phi_{n}=-\arg \left(t_{m n}\right)$. The target intensities before optimization $\left(I_{0}\right)$ and after an ideal optimization $\left(I_{\max }\right)$ are given by

$$
I_{0}=\frac{1}{N}\left|\sum_{n}^{N} t_{m n}\right|^{2},
$$

and

$$
I_{\max }=\frac{1}{N}\left(\sum_{n}^{N}\left|t_{m n}\right|\right)^{2} .
$$

For a disordered medium the elements of $t_{m n}$ are independent and have a Gaussian distribution.[20-23] Rewriting Eq. (7.5) gives

$$
\begin{aligned}
\left\langle I_{\max }\right\rangle & =\left\langle\frac{1}{N} \sum_{n, k \neq n}^{N}\left|t_{m n}\right|\left|t_{m k}\right|+\frac{1}{N} \sum_{n}^{N}\left|t_{m n}\right|^{2}\right\rangle, \\
& =\left\langle I_{0}\right\rangle\left[(N-1) \frac{\pi}{4}+1\right],
\end{aligned}
$$

where the angled brackets denote ensemble averaging over disorder. Eq. (7.7) predicts that the expected maximum enhancement for an ideally stable, noise free system linearly depends on the number of segments $N$. For $N \gg 1$, we have $\eta \approx \pi N / 4$.

\subsubsection{Performance in fluctuating environments}

In reality, the sample will not be completely stable. Whether this instability is due to a drift of the sample position, movement of the scatterers, changing humidity or 


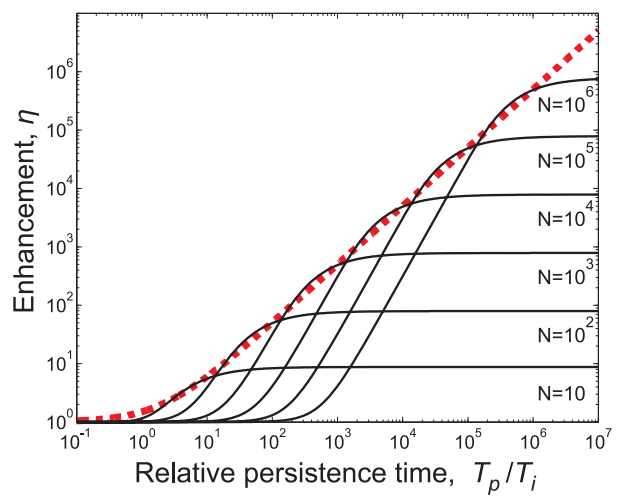

Figure 7.7: Theoretical maximum enhancement as a function of coherence time for different algorithms. The solid lines represent the maximum enhancement that can be obtained using the sequential algorithms. The enhancement depends on the number of segments used in the algorithm. The dashed line shows the enhancement for the partitioning algorithm where $N \gg T_{p} / T_{i}$.

any other cause, the transmission matrix will fluctuate over time. In the simulations, we modelled decoherence by repeatedly adding a small perturbation to each of the matrix elements.

$$
t_{m n} \rightarrow \frac{1}{\sqrt{1+\delta^{2}}}\left(t_{m n}+\xi\right)
$$

where $\xi$ is drawn from a complex Gaussian distribution with mean 0 and standard deviation $\delta$. The prefactor normalizes the transformation so that $\left\langle|t|^{2}\right\rangle$ remains constant. By substituting the continuous limit of Eq. (7.8) in Eq. (7.6), we find an analytic expression for the effect of decoherence,

$$
\left\langle I_{N}\right\rangle=\left\langle I_{0}\right\rangle\left[\frac{\pi}{4 N}\left(\sum_{n}^{N} e^{-T_{n} \delta^{2} /\left(2 T_{i}\right)}\right)^{2}+O(1)\right],
$$

where $T_{n}$ is the time that has past since the phase of segment $n$ was measured. This simple model explains the exponential decay of the intensity that was observed in the measurements (see Fig. 7.6a) and predicts a decay time of $T_{p}=T_{i} / \delta^{2}$.

We now calculate the maximum enhancement that can be reached with the continuous sequential algorithm in the presence of decoherence. Because the phases of the segments are measured sequentially, at any given time the values for $T_{n} / T_{i}$ are equally spaced between 1 and $N$. From Eq. (7.9) we find a maximum intensity enhancement of

$$
\eta_{N} \equiv \frac{\left\langle I_{N}\right\rangle}{\left\langle I_{0}\right\rangle}=\frac{\pi}{4 N}\left(\frac{1-e^{-N T_{i} /\left(2 T_{p}\right)}}{e^{T_{i} /\left(2 T_{p}\right)}-1}\right)^{2}+O(1) .
$$

The maximum enhancement for both sequential algorithms is the same. However, since the stepwise algorithm only updates the projected wavefront after $N$ iterations, the enhancement decreases exponentially between updates. 


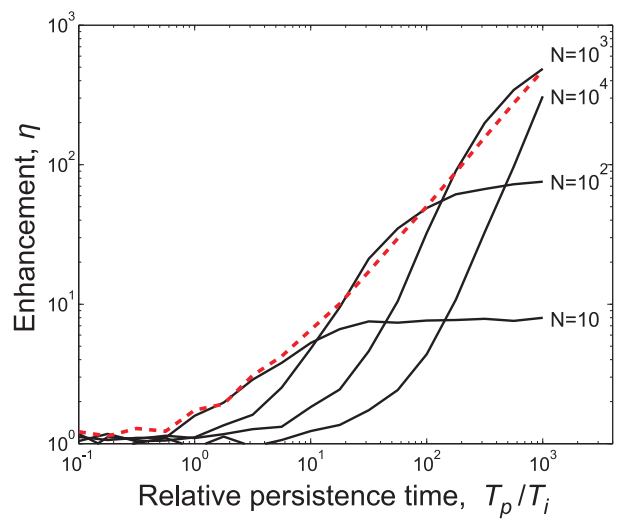

Figure 7.8: Simulated effect of decoherence on the sequential algorithms (solid curve) and on the partitioning algorithm (dashed curve). The simulations are averaged over 25 runs. Only when $N \approx 2.51 T_{p} / T_{i}$, the sequential algorithms perform slightly better than the partitioning algorithm.

In Fig. 7.7 the enhancement for different values of $N$ is plotted versus $T_{p} / T_{i}$. When the persistence time is large $\left(T_{p} / T_{i} \gg N\right)$, decoherence effects do not play a role and the enhancement linearly depends on $N$ as was seen in Eq. (7.7). For $T_{p} / T_{i}<N$, however, the enhancement decreases because the speckle pattern decorrelates before all iterations are performed and the enhancement drops to zero. As a consequence, the sequential algorithms only perform optimal when $N$ is adjusted to $T_{p}$. When $T_{p}$ is known a-priori, this optimum for $N$ can be found by maximizing Eq. (7.10). We find

$$
N_{\mathrm{opt}}=W T_{p} / T_{i} \text {, }
$$

where $W \approx 2.51$ is the solution of $\exp (W / 2)=1+W$. The maximal enhancement achievable with sequential algorithms follows by substituting Eq. (7.11) into Eq. (7.10) and equals $\eta_{\mathrm{opt}}=0.640 T_{p} / T_{i}$.

With the partitioning algorithm $\eta$ increases by $1 / 2$ each iteration of the algorithm (see Section 7.A). As long as $N \gg T_{p} / T_{i}$, the enhancement saturates at $\eta=T_{p} /\left(2 T_{i}\right)+1$, when the increase is exactly cancelled by the effect of decoherence. The most important difference with the sequential algorithms, is that the enhancement reached with the partitioning algorithm does not depend on N. In Fig. 7.7 it can be seen that the partitioning algorithm outperforms the sequential algorithms for almost all combinations of $T_{p}$ and $N$. The sequential algorithm only give a slightly higher enhancement when they are fine-tuned for a known persistence time $\left(N=2.51 T_{p} / T_{i}\right)$. In most situations, $T_{p}$ is not known a-priory or varies over time and the partitioning algorithm will be preferable.

Our analytical results for all three algorithms are supported by numerical simulations (see Fig. 7.8). The simulations exactly reproduce the theoretical curves shown in Fig. 7.7. For the simulations of the partitioning algorithm we used $N=4096$, a number that can easily be reached with a LCD phase modulator. Again, the partitioning algorithm can be seen to have good overall performance, whereas the sequential 


\begin{tabular}{|l|c|c|c|}
\hline & $\begin{array}{c}\text { stepwise } \\
\text { sequential }\end{array}$ & $\begin{array}{c}\text { continuous } \\
\text { sequential }\end{array}$ & partitioning \\
\hline signal & $2 I_{0} \sqrt{1 / N}$ & $2 I_{0} \sqrt{\eta / N}$ & $\eta I_{0}$ \\
bias & $I_{0}$ & $\eta I_{0}$ & $\eta I_{0}$ \\
relative shot noise SNR & $2 \sqrt{I_{0} / N}$ & $2 \sqrt{I_{0} / N}$ & $\sqrt{\eta I_{0}}$ \\
rms phase correction & $\sqrt{3} \pi$ & $\sqrt{3} \pi$ & $\sqrt{2 / \eta}$ \\
\hline
\end{tabular}

Table 7.1: Signal and noise characteristics of the three algorithms. The rms phase correction is a measure for the required sensitivity.

algorithms only work well for certain combinations of $N$ and $T_{p}$.

In conclusion, the maximum enhancement that can be reached linearly depends on the sample's persistence time. For the sequential algorithms $\eta=0.64 T_{p} / T_{i}$, but only when $N$ is precisely adjusted to $T_{p}$. The partitioning algorithm has $\eta=0.5 T_{p} / T_{i}$, as long as $N$ is large enough. Using these analytical relations, the performance of each of the three algorithms in different experimental situations can easily be estimated.

\subsection{Effect of Noise}

Measurement noise affects the measured phases. Noise-induced errors in the phases lead to a reduction of the enhancement, $\eta$. We will now compare the signal-to-noise ratio (SNR) of the three different algorithms. In a single iteration of an algorithm, the phase of one or more segments is varied, while the phase of the other segments is kept constant. The intensity at the detector equals

$$
I(\Phi)=I_{A}+I_{B}+2 \sqrt{I_{A} I_{B}} \cos \left(\Phi-\Phi_{0}\right)
$$

where $I_{B}$ is the intensity at the target originating from the modulated segments, $I_{A}$ is the target intensity caused by light coming from the other segments, $\Phi$ is the phase that is varied and $\Phi_{0}$ is the unknown optimal value for the phase. The last term in Eq. (7.12) is the signal that is relevant for measuring $\Phi_{0}$. There first two terms constitute a constant bias.

Table 7.1 lists the magnitudes of the signal and the bias for each of the three algorithms. If the detection system is photon shot noise limited, the noise is proportional to the square root of the bias. When, on the other hand, constant noise sources such as readout noise or thermal noise are dominant, the SNR is directly proportional to the signal magnitude. Table 7.1 also shows the root mean square (rms) phase correction that is applied during each iteration of the corresponding algorithm. The rms phase correction is a measure for the required accuracy of the measurements.

With the stepwise sequential algorithm, $I_{A} \approx I_{0}$ and on average $I_{B}=I_{0} / N$. Since the initial diffuse transmission $I_{0}$ is low and $N$ can be very high, the SNR is low. The continuous sequential algorithm has a higher SNR since the overall intensity at the 
detector increases while the algorithm progresses and $I_{A} \approx \eta I_{0}$. Assuming the dominant noise source is constant, the SNR will increase as the enhancement becomes higher. Therefore, the algorithm can be accelerated by decreasing the integration time of the camera as the algorithm advances. In case the detection system is photon shot noise limited, the SNR remains constant during the optimization since both the signal and the shot noise scale as $\sqrt{\eta I_{0}}$.

The highest SNR is achieved with the partitioning algorithm. Since we always change the phase of half of the segments, $I_{A} \approx I_{B} \approx \eta I_{0} / 2$, resulting in a maximal signal. Unlike the sequential algorithms, the SNR does not depend on $N$. Therefore the number of segments can be increased without suffering from noise. Like with the continuous sequential algorithm, the integration time can be adjusted dynamically to optimize the speed/SNR tradeoff. Although the partitioning algorithm has the highest SNR, the magnitude of the phase corrections decreases as the algorithm progresses. The required accuracy in measuring $\Phi_{0}$ increases at the same pace as the SNR increases.

The partitioning algorithm is very sensitive to measurement errors since, when the measured $\Phi_{0}$ has an error, half of the segments will be programmed with the wrong phase. In the extreme case where the error equals $\pi$ the enhancement completely disappears in a single iteration. A simple and effective solution to this problem is to keep the previous configuration of the phase modulator in memory. When an optimization step causes the signal to decrease, the algorithm can revert to the saved configuration.

\subsection{Simultaneously optimizing multiple targets}

For some applications one wishes to focus on multiple targets at once. To achieve multi-target focusing, one could use the sum of the intensities in all targets as feedback. In this case the feedback signal would equal (compare Eq. 7.12)

$$
\begin{aligned}
I_{\mathrm{M}}(\phi) & \equiv \sum_{m}^{M} I_{m}(\phi) \\
& =\sum_{m}^{M} I_{m A}+I_{m B}+2 \sqrt{I_{m A} I_{m B}} \cos \left(\Phi-\Phi_{m 0}\right),
\end{aligned}
$$

where the summation is over all $M$ targets. Each of the cosine terms in Eq. (7.14) is weighted by the square root of the intensity contribution of the modulated segments $I_{m B}$ and the contribution of the unmodulated segments $I_{m A}$. This weighting makes that targets that initially are more intense are optimized more effectively. While the intensity increases in all targets, the enhancement will not be distributed equally over the targets and the distribution depends on the starting conditions.

With the sequential algorithms, it is possible to distribute the enhancement equally over all targets by using a different feedback signal. If we use the summed square root 
of the intensities in the targets as feedback, we have a feedback 'amplitude'

$$
\begin{aligned}
A_{M}(\phi) & \equiv \sum_{m}^{M} \sqrt{I_{m}(\phi)} \\
& \approx \sum_{m}^{M} \sqrt{I_{m A}}+\sqrt{I_{m B}} \cos \left(\Phi-\Phi_{m 0}\right)
\end{aligned}
$$

where we used that $I_{m A} \gg I_{m B}$ for the sequential algorithms. For these algorithms, $I_{m A}$ is approximately equal to the initial intensity in target $m$. The feedback signal in Eq. (7.16) is only proportional to the propagation amplitude $\sqrt{I_{m B}}$ of the modulated segment to the target. Therefore, the signal does not depend on the initial conditions and the intensity enhancement will be distributed evenly over all targets.

\subsection{Conclusion}

Three different algorithms for inverting wave diffusion were presented. The algorithms were compared experimentally, with numerical simulations and using analytical theory. We found good agreement between experimental data, simulations and theory. Moreover, the simulations and theory can be used to predict the performance in different experimental situations.

The effectiveness of the algorithms was quantified by the enhancement. It was seen that the enhancement depends on the number of segments $N$ and the relative persistence time $T_{p} / T_{i}$. For the sequential algorithms to have optimal performance, it is required to adjust $N$ to match $T_{p}$. This means that these algorithms need a-priori knowledge of the system. The partitioning algorithm does not need this knowledge and always performs close to optimal. Moreover, the algorithm causes the enhancement to increase the most rapidly of the three investigated methods. All in all, this algorithm is a good candidate for applying inverse diffusion in instable scattering media such as living tissue. In the future, learning algorithms (see e.g. [24, 25]) might be developed to further improve the performance of inverse diffusion, for instance by dynamically balancing the trade-off between signal to noise ratio and measurement speed.

The maximum enhancement linearly depends on the number of measurements that can be performed before the speckle pattern decorrelates $\left(T_{p} / T_{i}\right)$. The faster the measurements, the higher the enhancement. In our current system, the speed is limited by the response time of the LCD. Fast micro mechanical phase modulators have a mechanical response time of about $10 \mu$ s (see e.g. [26]), which allows a $10^{4}$ times faster operation than with our current system. At such high speeds, the response time of the operating system of the control computer will become a bottleneck. Since the computation time required for the algorithm itself is negligible, this bottleneck can be overcome by using a real-time operating system, or by replacing the computer with a programmable logic device. In perfused tissue, a typical decorrelation timescale is $10 \mathrm{~ms}$ [16], which means that an enhancement of about 50 should be possible with currently available technology. 


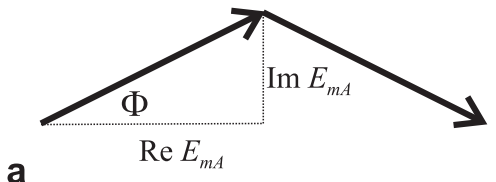

a

$\operatorname{Re} E_{m A}$

Figure 7.9: Complex plane representation of the partitioning algorithm. a) Before the iteration the contributions from $A$ and $B$ are not exactly in phase. b) After the iteration, the contributions are aligned and the resulting intensity is higher.

\section{A Calculation of the performance of the partitioning algorithm}

In this section we calculate the development of the enhancement of the partitioning algorithm under ideal conditions. During one iteration of the partitioning algorithm, the phase modulator is randomly split into two groups ( $A$ and $B$ ), each containing half of the segments. The relative phase $(\Phi)$ of group $B$ is cycled from 0 to $2 \pi$. During this cycle, the target intensity is given by

$$
I(\Phi)=\left|E_{m A}+E_{m B} e^{i \Phi}\right|^{2},
$$

where $E_{m A}$ is the contribution of the segments in group $A$ to the target field

$$
E_{m A}=\sum_{n \in A} \sqrt{\frac{\left\langle I_{0}\right\rangle}{N}} \xi_{m n},
$$

with

$$
\xi_{m n} \equiv \sqrt{\frac{1}{\left\langle I_{0}\right\rangle}} t_{m n} e^{i \phi_{n}},
$$

and similar for $E_{m B}$. The coefficients $\xi_{m n}$ are initially random and distributed according to a normalized circular Gaussian distribution, meaning that $\langle\xi\rangle=0$ and $\left\langle(\operatorname{Re} \xi)^{2}\right\rangle=\left\langle(\operatorname{Im} \xi)^{2}\right\rangle=1 / 2$. As the algorithm proceeds, the phases $\phi_{n}$ are adjusted and the distribution gradually changes to a Rayleigh distribution when a high enhancement is reached. The average value $\langle\xi\rangle$ increases from 0 to $\sqrt{\pi / 4}$ as all contributions are aligned to be in phase. At any moment during the optimization, $\langle\xi\rangle=$ $\sqrt{(\eta-1) /(N-1)}$ and $\left\langle|\xi|^{2}\right\rangle=1$.

Figure 7.9 gives a graphical representation of a single iteration. Before the iteration, $E_{m A}$ and $E_{m B}$ have a different phase. Without loss of generality, we choose the phase of $\left(E_{m A}+E_{m B}\right)$ to be 0 . The intensity before the iteration is given by

$$
I_{\text {before }}=\left(\operatorname{Re} E_{m A}+\operatorname{Re} E_{m B}\right)^{2} .
$$

After the iteration, $\Phi$ is set to the value that caused the highest target intensity, which means that $E_{m A}$ and $E_{m B}$ are now in phase. The target intensity then equals

$$
I_{\mathrm{after}}=\left(\left|E_{m A}\right|+\left|E_{m B}\right|\right)^{2},
$$

which is higher than or equal to before the iteration. 
We now calculate the average intensity gained in a single iteration. We consider the regime where already a few iterations have been done $(\eta \gg 1)$. In this regime, we can approximate

$$
\begin{aligned}
\left|E_{m A}\right| & =\sqrt{\left(\operatorname{Re} E_{m A}\right)^{2}+\left(\operatorname{Im} E_{m A}\right)^{2}} \\
& \approx \operatorname{Re} E_{m A}+\frac{\left(\operatorname{Im} E_{m A}\right)^{2}}{2 \operatorname{Re} E_{m A}} .
\end{aligned}
$$

Using this result in Eq. (7.21) gives

$$
\begin{aligned}
& I_{\text {after }}=\left(\operatorname{Re} E_{m A}+\operatorname{Re} E_{m B}\right)^{2}+\left(\operatorname{Im} E_{m A}\right)^{2}+\left(\operatorname{Im} E_{m B}\right)^{2}+ \\
&+\frac{\operatorname{Re} E_{m A}}{\operatorname{Re} E_{m B}}\left(\operatorname{Im} E_{m B}\right)^{2}+\frac{\operatorname{Re} E_{m B}}{\operatorname{Re} E_{m A}}\left(\operatorname{Im} E_{m A}\right)^{2}+ \\
& \quad+\frac{\left(\operatorname{Im} E_{m A}\right)^{4}}{4\left(\operatorname{Re} E_{m A}\right)^{2}}+\frac{\left(\operatorname{Im} E_{m B}\right)^{4}}{4\left(\operatorname{Re} E_{m B}\right)^{2}}+\frac{\left(\operatorname{Im} E_{m A} \operatorname{Im} E_{m B}\right)^{2}}{2 \operatorname{Re} E_{m A} \operatorname{Re} E_{m B}}
\end{aligned}
$$

where the terms on the last line can be neglected. If $N \gg 1$, then $\operatorname{Re} E_{m B} / \operatorname{Re} E_{m A} \approx 1$. The intensity gain for the iteration $\Delta I \equiv I_{\text {after }}-I_{\text {before }}$ is now found to be

$$
\Delta I=2\left(\operatorname{Im} E_{m A}\right)^{2}+2\left(\operatorname{Im} E_{m B}\right)^{2}
$$

We are primarily interested in the regime $N \gg \eta \gg 1$ ), where the algorithm picks up the main part of the final enhancement. In this regime, $\langle\xi\rangle \ll 1$ and the probability distribution of $\xi$ is still close to the original Gaussian distribution. Therefore, $\left\langle(\operatorname{Im} \xi)^{2}\right\rangle \approx 1 / 2$ and it follows from Eq. (7.18) that

$$
\Delta I=\frac{1}{2}\left\langle I_{0}\right\rangle
$$

Therefore, we expect the intensity enhancement $\eta$ to increase with $1 / 2$ after each iteration of the algorithm. With this information, we also calculate the typical phase adjustment that is performed in each iteration. From Fig. 7.9 it follows that the root mean square phase adjustment equals

$$
\Phi_{\mathrm{rms}} \equiv \sqrt{\left\langle\Phi^{2}\right\rangle}=\sqrt{\left\langle\frac{\left(\operatorname{Im} E_{m A}\right)^{2}}{\left(\operatorname{Re} E_{m A}\right)^{2}}\right\rangle}=\sqrt{\frac{2}{\eta}}
$$

When $\eta$ approaches its maximum, all contributions are almost completely in phase and $\left\langle(\operatorname{Im} \xi)^{2}\right\rangle$ vanishes. In this regime, the algorithm becomes less and less effective, as was seen in simulations and experiments (see Fig. 7.6). Adaptive switching to one of the other algorithms will ensure optimal convergence.

\section{Bibliography}

[1] E. A. Milne, Radiative equilibrium in the outer layers of a star, Monthly Not. Roy. Astron. Soc. 81, 361 (1921).

[2] S. Chandrasekhar, Radiative transfer (Dover Publications, Inc., New York, 1960). 
[3] A. Ishimaru, Limitation on image resolution imposed by a random medium, Appl. Opt. 17, 348 (1978).

[4] I. M. Vellekoop and A. P. Mosk, Focusing coherent light through opaque strongly scattering media, Opt. Lett. 32, 2309 (2007), Chapter 3 of this thesis.

[5] R. K. Tyson, Principles of adaptive optics, 2nd ed. (Academic Press, New York, 1998).

[6] M. Fink, D. Cassereau, A. Derode, C. Prada, P. Roux, M. Tanter, J.-L. Thomas, and F. Wu, Time-reversed acoustics, Rep. Prog. Phys. 63, 1933 (1999).

[7] O. I. Lobkis and R. L. Weaver, On the emergence of the Green's function in the correlations of a diffuse field, J. Ac. Soc. Am. 110, 3011 (2001).

[8] L. Borcea, G. Papanicolaou, and C. Tsogka, Interferometric array imaging in clutter, Inv. Prob. 21, 1419 (2005).

[9] G. Lerosey, J. de Rosny, A. Tourin, and M. Fink, Focusing beyond the diffraction limit with far-field time reversal, Science 315, 1120 (2007).

[10] G. J. Foschini, Layered space-time architecture for wireless communication in a fading environment when using multi-element antennas, Bell Labs Technical Journal 1, 41 (1996).

[11] N. M. Shapiro, M. Campillo, L. Stehly, and M. H. Ritzwoller, High-resolution surface-wave tomography from ambient seismic noise, Science 307, 1615 (2005).

[12] W. A. Kuperman, W. S. Hodgkiss, H. C. Song, T. Akal, C. Ferla, and D. R. Jackson, Phase conjugation in the ocean: Experimental demonstration of an acoustic time-reversal mirror, J. Acoust. Soc. Am. 103, 25 (1998).

[13] M. I. Stockman, S. V. Faleev, and D. J. Bergman, Coherent control of femtosecond energy localization in nanosystems, Phys. Rev. Lett. 88, 067402 (2002).

[14] J. L. Herek, W. Wohlleben, R. J. Cogdell, D. Zeidler, and M. Motzkus, Quantum control of energy flow in light harvesting, Nature 417, 533 (2002).

[15] R. Pappu, B. Recht, J. Taylor, and N. Gershenfeld, Physical one-way functions, Science 297, 2026 (2002).

[16] J. D. Briers and S. Webster, Quasi real-time digital version of single-exposure speckle photography for full-field monitoring of velocity or flow fields, Opt. Commun. 116, 36 (1995).

[17] R. H. J. Kop, P. de Vries, R. Sprik, and A. Lagendijk, Observation of anomalous transport of strongly multiple scattered light in thin disordered slabs, Phys. Rev. Lett. 79, 4369 (1997).

[18] J. A. Davis, J. Nicolás, and A. Márquez, Phasor analysis of eigenvectors generated in liquidcrystal displays, Appl. Opt. 41, 4579 (2002).

[19] J. Reményi, P. Várhegyi, L. Domján, P. Koppa, and E. Lõrincz, Amplitude, phase, and hybrid ternary modulation modes of a twisted-nematic liquid-crystal display at $400 \mathrm{~nm}$, Appl. Opt. 42, 3428 (2003).

[20] J. W. Goodman, Statistical optics (Wiley, New York, 2000).

[21] N. Garcia and A. Z. Genack, Crossover to strong intensity correlation for microwave radiation in random media, Phys. Rev. Lett. 63, 1678 (1989).

[22] M. A. Webster, T. D. Gerke, A. M. Weiner, and K. J. Webb, Spectral and temporal speckle field measurements of a random medium, Opt. Lett. 29, 1491 (2004).

[23] C. W. J. Beenakker, Random-matrix theory of quantum transport, Rev. Mod. Phys. 69, 731 (1997).

[24] D. E. Goldberg, Genetic algorithms in search, optimization \& machine learning (AddisonWesley, Reading, MA, 1989).

[25] R. S. Judson and H. Rabitz, Teaching lasers to control molecules, Phys. Rev. Lett. 68, 1500 (1992).

[26] M. Hacker, G. Stobrawa, R. Sauerbrey, T. Buckup, M. Motzkus, M. Wildenhain, and A. Gehner, Micromirror SLM for femtosecond pulse shaping in the ultraviolet, Appl. Phys. B 76, 711 (2003). 
102 | Bibliography 


\section{Chapter 8}

\section{Transport of light with an optimized wavefront}

In our experiments we construct a wavefront that maximizes the intensity at a given target position. In this chapter, we investigate the transport properties of light with such an optimized wavefront. We introduce the concept of 'active' measurements to describe a measurement where the wavefront is tailored to the specific realization of disorder in the sample. We show that this new type of experiments is very promising as it could be used to find signatures of absorption and possibly Anderson localization of light, or to test mesoscopic transport theories and selectively study extended modes in a sample.

So far, we have used a simple ad hoc matrix model (Eq. (3.1)) to describe scattering and focusing through a disordered medium. This model is based on the assumption of uncorrelated transmission coefficients (UTC). In reality, the constraint on energy conservation makes that the matrix elements are correlated. Such correlations can be described by random matrix theory (RMT). RMT predicts that it is possible to generate an incident wavefront that is fully transmitted through an opaque object, regardless of the thickness of the object.

In Section 8.1 we give a brief introduction to RMT and discuss how it can be applied to describe scattering in a disordered optical waveguide. In Section 8.2 we use RMT to describe the transport of light in an active measurement. We calculate experimentally observable quantities and compare the situations with the matched and unmatched incident wavefronts. Furthermore, we discuss the physical relevance of these 'actively measured' quantities and compare the results with predictions made with the UTC model. The total transmission of an optimized wavefront is found to be an observable that characterizes the correlations in the scattering matrix. Finally, in Section 8.3, we extend the RMT model so that it applies to a slab geometry and accounts for other experimental parameters, such as the resolution of the light modulator. We use the extended model to calculate how experimental limitations affect the measurements and we show how they can be compensated for.

\subsection{Random matrix theory}

Random matrix theory is a very general mathematical theory for statistically describing extremely complex systems. Some excellent reviews on RMT can be found in Refs. 1, 2 and 3. The theory has been developed by Wigner[4] to describe statistics of eigenvalues of many-body systems. It was originally employed to describe the properties of excited states of atomic nuclei and also proved very successful in calculating the statistics of electron scattering in mesoscopic conductors and quan- 


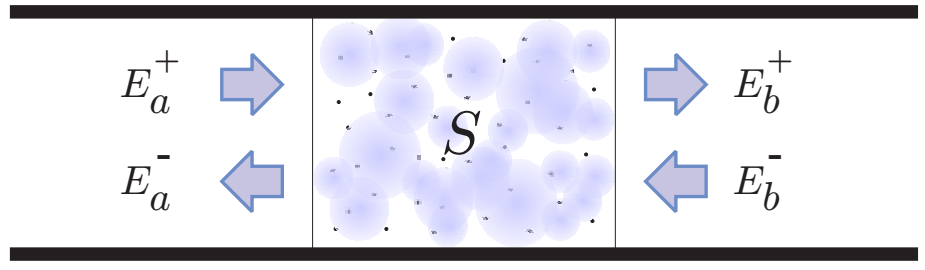

Figure 8.1: Scattering in a waveguide geometry. The scattering matrix $S$ couples incident waveguide modes $E_{a}^{+}$and $E_{b}^{-}$to outgoing waveguide modes $E_{a}^{-}, E_{b}^{+}$. In a transmission experiment, $E_{b}^{-}=0$.

tum dots[5-8]. Weaver[9] was the first to study the application of RMT to acoustical waves instead of quantum mechanical wave functions. In pioneering experiments in acoustics $[9,10]$ and microwaves $[11,12]$ the distribution of eigenfrequencies in irregular cavities was measured. RMT proved very successful in predicting the statistical properties of eigenfrequency spacing in these systems.[3] Although most results of RMT are for monochromatic waves and, therefore, do not describe the dynamics of scattering, methods for extending the matrix treatment to describe time dependence are being developed[13-16].

More recently, RMT has been used to calculate correlations of light scattering in disordered optical waveguides (see e.g. [2]) and random lasers[17]. Because only very general concepts are used, it is very easy to translate results for one type of waves to a different system. This also means that experimental observations in one field of physics are directly relevant for many other disciplines.

The basic assumption of RMT is that the system is so complex that you might as well use a completely random Hamiltonian matrix to describe it. In Wigner's original approach[4], the 'randomness' of the matrix is invariant under unitary transformations of Hilbert space. In terms of light scattering, this invariance means that the scattered light has no spatial or angular correlation with the incident light whatsoever. The only other constraint that Wigner imposed on the random matrices is that there is a conserved quantum number that imposes a symmetry condition on the matrix. In the case of scattering this condition is that the energy is conserved (no absorption). Usually, an optical system also has time-reversal symmetry, which puts an extra constraint on the random matrix. Due to these constraints the elements of the matrix have to be statistically correlated. Typically, the number of scattering channels is large and the correlation between any pair of channels is negligible. Only when many scattering channels are involved, the effects of the correlations become visible.

\subsubsection{Random matrix theory in a waveguide geometry}

We first explain how RMT is applied to a simple waveguide geometry as is shown in Fig. 8.1. To both sides of a strongly scattering disordered sample a waveguide supporting exactly $N$ propagating waveguide modes (channels) is connected. Evanescent fields in the waveguide are not taken into consideration. The sample scatters incident light into reflected and transmitted waveguide channels. 


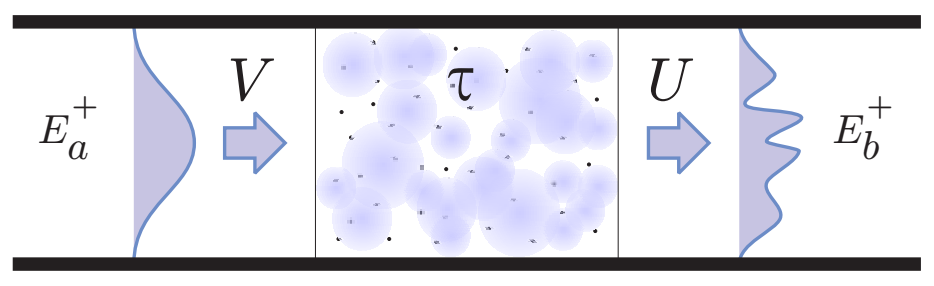

Figure 8.2: Transmission experiment in a waveguide geometry. The transmission matrix of the system is decomposed in three matrices: $V$ maps the incident light to eigenmodes of the sample, $\tau$ gives amplitude transmission coefficients for the eigenmodes. $U$ maps the eigenmodes back to real space on the other side of the sample.

Scattering by the sample is represented by the scattering matrix $S$ that connects the incident field $E_{a}^{+}, E_{b}^{-}$to the channels leaving the sample $\left(E_{a}^{-}\right.$and $\left.E_{b}^{+}\right)$. Channels labelled $a$ are channels on the left side of the sample and Channels labelled $b$ are channels on the right side of the sample. The scattering matrix $S$ is defined as

$$
\left[\begin{array}{c}
E_{a}^{-} \\
E_{b}^{+}
\end{array}\right]=S\left[\begin{array}{c}
E_{a}^{+} \\
E_{b}^{-}
\end{array}\right] .
$$

We assume that the sample does not absorb any light and, therefore, $S$ is unitary. When, in addition, scattering in the sample is reciprocal, time-reversal symmetry is obeyed and $S$ is both unitary and symmetric. Although all our experimental systems have time-reversal symmetry, unitarity alone is sufficient for the derivations made in this chapter to be valid. The scattering matrix can be split into four matrices describing reflection and transmission

$$
\left[\begin{array}{c}
E_{a}^{-} \\
E_{b}^{+}
\end{array}\right]=\left[\begin{array}{cc}
r_{a a}^{-+} & t_{a b}^{--} \\
t_{b a}^{++} & r_{b b}^{+-}
\end{array}\right]\left[\begin{array}{c}
E_{a}^{+} \\
E_{b}^{-}
\end{array}\right] .
$$

From now on, we only consider transmission from left (channels $a$ ) to right (channels $b$ ) as described by the matrix $t_{b a}^{++}$, in short $t$. RMT tells us that the restriction of energy conservation gives rise to a statistical structure in the transmission matrix. This structure is visible in the singular value decomposition (SVD) of the transmission matrix. Like any matrix, the transmission matrix $t$ can be decomposed as

$$
t=U \tau V
$$

where $U$ and $V$ are unitary matrices and $\tau$ is a diagonal matrix with real, non-negative elements. The physical interpretation of Eq. (8.3) is illustrated in Fig. 8.2. Matrix $V$ maps the incident light from real space coordinates onto transmission eigenmodes of the sample. The intensity transmission coefficients for each of these eigenmodes are given by the diagonal elements of $\tau^{2}$. Matrix $U$ maps the eigenmodes back to spatial coordinates at the back of the sample.

In RMT $U$ and $V$ are random unitary matrices with elements that have a circular Gaussian distribution. The statistical properties of these matrices are invariant to any 


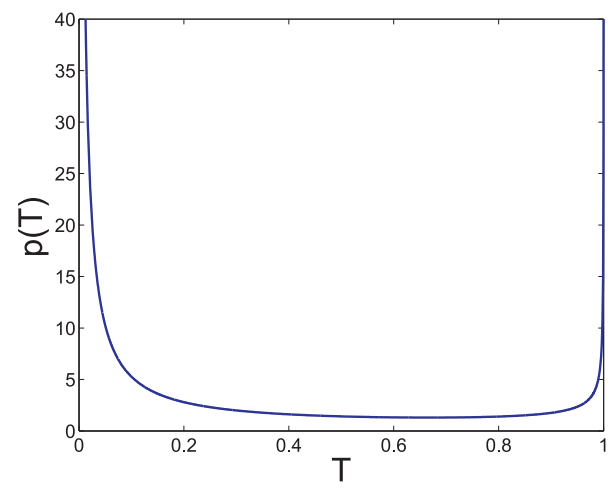

Figure 8.3: Probability density of the intensity transmission eigenvalues $T \equiv \tau^{2}$ as derived in Ref. 5 .

unitary transform of the Hilbert space.[4] In other words, when we replace $U$ (or $V$ ) by $U^{\prime}=B U\left(V^{\prime}=B V\right)$, with $B$ any unitary matrix, the ensemble properties do not change. It, therefore, does not matter whether a channel is defined as a waveguide mode, an incident plane wave or a position on the sample surface. Moreover, these constraints on $U$ and $V$ make that these matrixes are statistically independent of $\tau$.

\subsubsection{Distribution of transmission eigenvalues}

Intuitively, one might expect that all modes in a random system are roughly equivalent and the distribution of $T \equiv \tau^{2}$ is peaked around the total transmission of the sample. However, as was shown first by Dorokhov[5], the eigenvalues have a probability density that is given by

$$
p(T)=\langle T\rangle \frac{1}{2 T \sqrt{1-T}} .
$$

This probability density is plotted in Fig. 8.3. The eigenvalue distribution is bimodal: almost all eigenvalues are either 1 or 0 . A common terminology is to speak of 'open' and 'closed' channels. Light in an open channel is almost fully transmitted $(T \approx 1)$, whereas light in closed channels is almost completely reflected $(T \approx 0)$. The prediction that all channels are either open or closed is also known as the maximal fluctuation theorem $[18,19]$.

The distribution in Eq. (8.4) is the result of the restriction of energy conservation that is imposed on the scattering matrix. Due to this restriction, a mechanism called eigenvalue repulsion spreads out the values of $1 / \xi$ to a uniform distribution.[2, 18, 20] Here, $\xi$ is the localization length that is associated with a mode in the sample. The localization length relates to the transmission of a sample by $T=\cosh ^{-2}(L / \xi)$. The uniform distribution of $1 / \xi$ transforms into the distribution of $T$ as is given by Eq. (8.4). Originally, RMT was used to derive Eq. (8.4) for a waveguide. However, later it was shown that this probability density function is more generally valid and also applies to a slab geometry.[21] 
Equation (8.4) has divergencies at 0 and at 1 . The divergency at 0 is not integrable. In fact, there is a minimum value for the transmission coefficient of a mode[2, 22], which is given by $T=\cosh ^{-2}(1 /\langle T\rangle)$. This cutoff normalizes the probability distribution of $T$. The physical interpretation of the cutoff is that with $\langle T\rangle \approx \ell / L$, the localization length of a mode cannot be shorter than the mean free path $\ell$. In practice, the cutoff for $T$ is often ignored since most of the time one is only interested in the moments of $T$. These moments depend very little on the cutoff.[22]

\subsubsection{The effect of refractive indices}

Random matrix theory is most conveniently formulated in a waveguide geometry with an equal number of waveguide channels on both side of the sample. In an experiment, the refractive index in front of the sample is often different from the refractive index at the back. Moreover, the sample itself has an effective refractive index that may differ from that of its environment. The number of channels scales with the refractive index squared. Therefore, the number of channels in the sample is not the same as the number of channels in the connected waveguides.

To account for the different number of scattering channels on both sides of the medium we assume that all channels are statistically equivalent. The number of channels is determined by the refractive index in each of the media. At an interface, scattering randomly redistributes the light over all channels. When the light enters a medium with a lower refractive index, some linear combinations of channels are completely reflected. This reflection is the random equivalent of total internal reflection.

In general the transmission coefficient at the surface of a disordered medium is angle dependent[23-25]. If we define channels as incident angles, different channels are not statistically equivalent. Therefore, we chose to define channels in real space coordinates. Since angular effects are equal for all channels, the channels are still statistically equivalent. Furthermore, we assume that the refractive index contrasts are low and that scattering is strong, so that neighboring channels can be considered statistically independent.

We now consider three possible combinations of refractive indices and analyze their effect on the RMT formalism.

\section{Matrix description of a sample in air}

When a sample is surrounded by air, its effective refractive index is higher than that of the surrounding medium. This means that the sample supports more channels than can possibly couple to the outside world. The difference in supported channels is shown schematically in Fig. 8.4. Since no light can couple to these bound modes, we ignore them and just use the formalism in Eq. (8.3), with $\tau$ a random subset of the actual full $\tau$ matrix of the sample.

\section{Matrix description of a sample in a dense medium}

In the situation where the refractive index of the sample is lower than that of its environment not all incident channels can couple into the sample. Or, more correctly, 


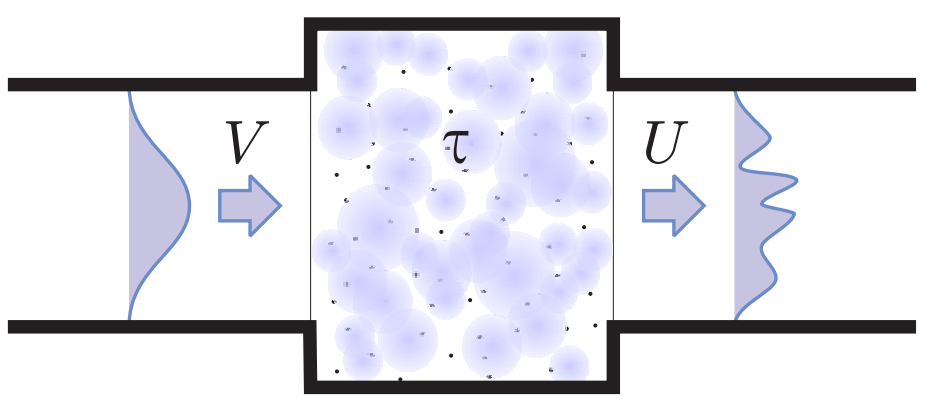

Figure 8.4: Diagrammatic representation of a sample in a medium with a lower refractive index. The sample supports more channels than the connecting waveguides and can, be thought of in a sense being 'wider'.

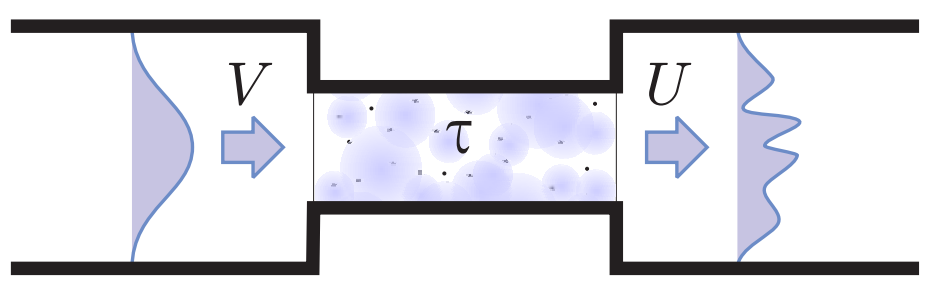

Figure 8.5: Diagrammatic representation of a sample in a medium with a higher refractive index. The sample supports fewer channels than the connecting waveguides and can, be thought of in a sense being 'narrower'. Not all incident channels can couple into the sample.

some linear combinations of incident channels have no support in the sample. As is depicted in the diagram in Fig. 8.5, the sample acts as a bottleneck. This situation could be modelled by using the formalism in Eq. (8.3) with a matrix $\tau$ that is extended with extra zeros for channels that cannot exist inside the sample.

\section{Matrix description of a sample on a substrate}

When the sample is on a substrate with a high refractive index, it is sandwiched between a low refractive index medium and a high refractive index medium. In this experimentally relevant situation, the number of incident channels is not equal to the number of transmitted channels and $t$ is a rectangular $M \times N$ matrix. Here $N$ is the number of incident channels and $M$ is the number of transmitted channels. In the singular value decomposition of $t, U$ is a unitary $M \times M$ matrix, $V$ is a unitary $N \times N$ matrix, and $\tau$ is a rectangular diagonal $M \times N$ matrix. The number of diagonal elements in $\tau$ equals $K \equiv \min (M, N)$. When the sample is illuminated through the low index waveguide, as is shown in Fig. 8.6, there will be no total internal reflection as the light travels from a low refractive index towards a high refractive index. Since the case with a rectangular $t$ matrix is the most general, we will use this case in our derivations. 


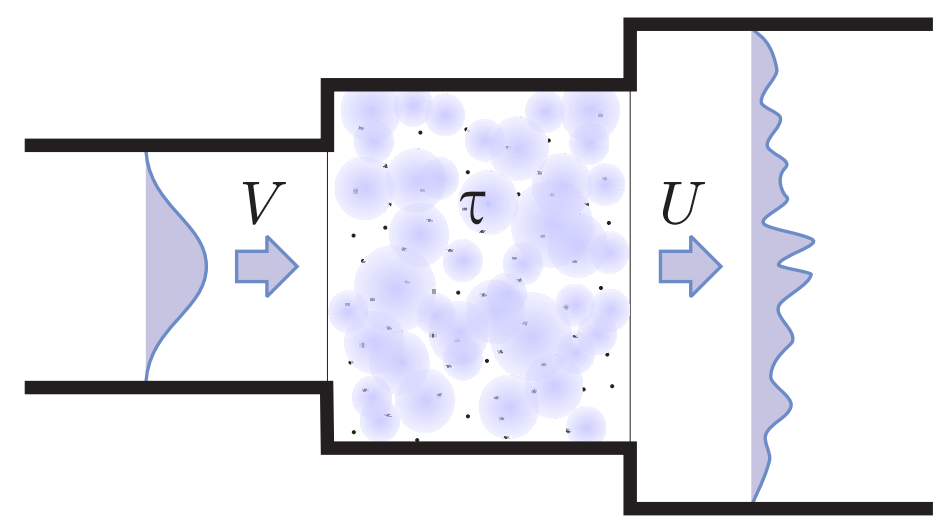

Figure 8.6: Diagrammatic representation of a sample on a substrate with a higher refractive index than that of the sample. All channels on the low index side of the sample can couple to the sample and, in turn, all channels in the sample can couple to the substrate.

\subsection{A new class of experimental observables}

A common way to experimentally study an optical system is to measure its response to a fixed incident wavefront. For a disordered system, the response will be stochastic. Useful results are obtained by statistical methods such as ensemble averaging and calculating correlation functions.

In our experiment, however, we actively adapt the incident wavefront to each specific realization of disorder in the sample and selectively excite an extended optical mode. This concept is analogous to the approach taken in the field of coherent control, where a femtosecond pulse is shaped in the frequency domain to optimize the response of a chemical system[26-31]. This pulse selectively excites a chemical pathway that can then be studied. For most systems described by RMT such control is not possible and at best ensemble averaged quantities can be observed.

One of the potentials of our method becomes apparent from the distribution $p(T)$ in Eq. (8.4). This equation predicts that there always exist channels that have $T=1$. Using a wavefront synthesizer we can, in principle, generate an incident wavefront that only couples to these open channels. Such a wavefront should be fully transmitted through an otherwise opaque scattering medium.

Open channels correspond to optical modes that extend over the whole thickness of the sample.[2] With our active measurement method, it is possible to selectively study the properties (correlations, speckle statistics, delay time statistics, behavior in random gain media, second harmonic generation, etcetera) of extended modes without having a contribution from the localized modes that correspond to closed channels. The relation between localized modes and extended modes is of paramount importance in the study of Anderson localization (where the number of extended modes vanishes, see e.g. [2]) and in the field of random lasers[32].

The possibility to perform active measurements once again illustrates the fundamental difference between having a light modulator in front of the sample or behind the sample. The two situations are shown in Fig. 8.7. In Fig. 8.7a the modulator is 
a

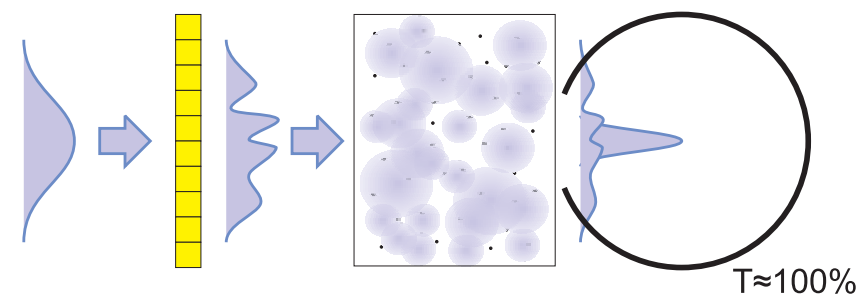

b
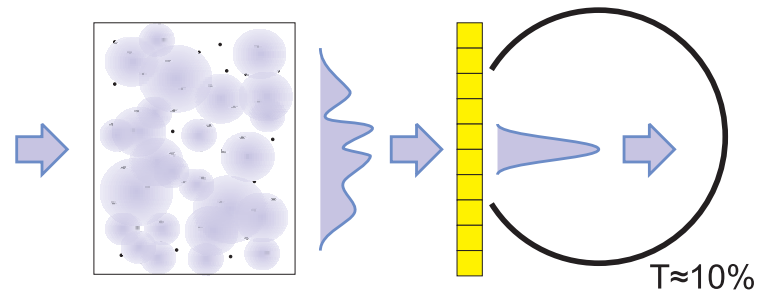

Figure 8.7: Difference between placing a light modulator in front of the sample or behind. a) Light modulator in front of the sample. A sharp focus surrounded by a speckle pattern is formed and the total transmission in the order of $100 \%$. b) Light modulator behind the sample. All transmitted light is formed into a focus without speckle. The total transmission does not change and typically is in the order of $10 \%$.

placed in front of the sample and the incident wavefront is optimized for creating a focus behind the sample. The optimal wavefront only couples to extended modes in the sample and the total transmission increases. Since only a fraction of all channels is open, there is no complete set of basis vectors for forming any desired field behind the sample. As a result, it is not possible to concentrate all light in the focus; the rest of the transmitted light will form a random speckle pattern. When a modulator is placed behind the sample, as is shown in Fig. 8.7b, it could in principle focus all transmitted light to a single point. However, light transport in the sample is, of course, not affected.

To find a wavefront that excites only open channels, one could theoretically perform a singular value decomposition of the full transmission matrix (see Eq. (8.3)). However, measuring the complete scattering matrix is experimentally very challenging since it contains tens of millions of elements. Moreover, measurement noise would accumulate and disturb the singular value decomposition. A different, much easier, approach is to create a focus at any position behind the sample. Since closed channels are fully reflected, only open channels contribute to this focus. When we optimize the incident light to create a maximally intense focus, we generate a wavefront that only overlaps with open channels.

In this section, we use the RMT model to calculate some experimentally observable quantities and compare the result for an unshaped wavefront (passive measurement) to the result of a wavefront that is optimized for creating a focus (active measurement). The results of the calculations that follow below are summarized in Table 8.1. We first derive these quantities for a waveguide geometry with a wavefront synthesizer that perfectly controls the phase and amplitude of all incident channels. 


\begin{tabular}{|l|c|c|}
\hline & passive & active (optimized) \\
\hline single channel & $\left\langle I_{\beta}\right\rangle=\left\langle\tau^{2}\right\rangle \frac{K}{M N}$ & $\left\langle\widetilde{I}_{\beta}\right\rangle=\left\langle\tau^{2}\right\rangle \frac{K}{M}$ \\
total & $\left\langle T_{\text {tot }}\right\rangle=\left\langle\tau^{2}\right\rangle \frac{K}{N}$ & $\left\langle\widetilde{T}_{\text {tot }}\right\rangle=\frac{2}{3}$ \\
\hline
\end{tabular}

Table 8.1: Average transmission into a single channel and average total transmission with and without actively shaped wavefront.

This assumption greatly simplifies the calculations. Later, in Section 8.3, we will see how the general results for an open system or for imperfect modulation can easily be found once the results for a perfect waveguide system are known.

\subsubsection{Observables in passive measurements}

We now calculate average values for the total transmission through the sample and for the intensity in a single transmission channel. The incident wavefront is not adapted to the sample, which is the normal, passive, way of measuring transmission coefficients. The general equation for calculating the field in outgoing channel $b$ is

$$
E_{b}=\sum_{a}^{N} t_{b a} E_{a}
$$

Using the singular value decomposition in Eq. (8.3), we obtain

$$
E_{b}=\sum_{a}^{N} \sum_{k}^{K} U_{b k} \tau_{k k} V_{k a} E_{a} .
$$

Because RMT is symmetric to unitary transformations of the incident field, we can use any arbitrary normalized field and achieve the same outcome. For simplicity, we illuminate only incident channel $a^{\prime}$ and have $E_{a}=\delta_{a^{\prime} a}$. The total transmission is calculated using

$$
T_{\text {tot }} \equiv \sum_{b}^{M} I_{b}
$$

with $I_{b} \equiv\left|E_{b}\right|^{2}$. The total transmission follows from substituting Eq. (8.6) into Eq. (8.7)

$$
T_{\text {tot }}=\sum_{b}^{M}\left|\sum_{k}^{K} U_{b k} \tau_{k k} V_{k a^{\prime}}\right|^{2} .
$$

After expanding the square and reordering terms, we have

$$
T_{\text {tot }}=\sum_{b}^{M} \sum_{k, k^{\prime}}^{K} U_{b k}^{*} U_{b k^{\prime}} \tau_{k k} \tau_{k^{\prime} k^{\prime}} V_{k a^{\prime}} V_{k^{\prime} a^{\prime}}^{*}
$$

Unitarity of $U$ implies that $\sum_{b}^{M} U_{b k}^{*} U_{b k^{\prime}}=\delta_{k k^{\prime}}$. Therefore, Eq. (8.9) reduces to

$$
T_{\text {tot }}=\sum_{k}^{K} \tau_{k k}^{2}\left|V_{k a^{\prime}}\right|^{2}
$$


To find the ensemble average total transmission, we use the assumption from RMT that $V$ and $\tau$ are statistically independent. We can, therefore, average Eq. (8.10) over all realizations of disorder to find

$$
\left\langle T_{\text {tot }}\right\rangle=\left\langle\sum_{k}^{K} \tau_{k k}^{2}\left|V_{k a^{\prime}}\right|^{2}\right\rangle=\left\langle\tau^{2}\right\rangle\left\langle\sum_{k}^{K}\left|V_{k a^{\prime}}\right|^{2}\right\rangle=\left\langle\tau^{2}\right\rangle \frac{K}{N} .
$$

where the unitarity of $V$ was used in the final step. When the refractive index behind the sample is higher than the refractive index in front of the sample, the factor $K / N$ equals one. On average, an incident field excites all eigenmodes with equal probability. Therefore, the average total transmission equals $\left\langle\tau^{2}\right\rangle$.

If, on the other hand the refractive index behind the sample is lower, the factor $K / N$ is less than one. To understand this factor, we examine the situation where there is no sample, just a direct transition between the two media. When $N>M$, there are more channels in the incident field than there are in the transmitted field. The excess channels cannot exist in the medium behind the sample and undergo total internal reflection. The fraction of channels that does not experience total internal reflection is $M / N$. When a sample is present, energy is distributed randomly over all sample channels. Only a fraction $M / N$ of the light can leave the sample.

Since in the RMT formalism all transmitted channels are equivalent, the average intensity in a single transmitted channel is simply found by dividing Eq. (8.11) by $M$

$$
\left\langle I_{b}\right\rangle=\left\langle\tau^{2}\right\rangle \frac{K}{M N}
$$

\subsubsection{Observables in active measurements}

We now calculate the same quantities as in the previous section for an active measurement where the incident wavefront is optimized to maximize transmission into a single channel $\beta$. We first determine this optimal incident field $\widetilde{E}_{a}$ and then use it to calculate the transmission into channel $\beta$ as well as the total transmission.

\section{Intensity in the optimized channel}

We now calculate the intensity in the target channel $\beta$ after an optimization. The incident field that maximizes the intensity in this channel follows from the CauchySchwartz inequality

$$
I_{\beta}=\left|\sum_{a}^{N} t_{\beta a} E_{a}\right|^{2} \leq \sum_{a}^{N}\left|t_{\beta a}\right|^{2} \sum_{a^{\prime}}^{N}\left|E_{a^{\prime}}\right|^{2},
$$

where the two sides are equal if and only if

$$
\widetilde{E}_{a}=A_{0} t_{\beta a}^{*}
$$

where we denoted the optimal incident field after optimization with a tilde sign. We are free to choose prefactor $A_{0} \in \mathbb{C}$; we choose $A_{0}$ so that $\widetilde{E}_{a}$ is normalized. Now, the 
optimal value of $I_{\beta}$ follows directly from Eq. (8.13)

$$
\widetilde{I}_{\beta}=\sum_{a}^{N}\left|t_{\beta a}\right|^{2}
$$

Using the singular value decomposition in Eq. (8.3), we find

$$
\begin{aligned}
\widetilde{I}_{\beta} & =\sum_{a}^{N}\left|\sum_{k}^{K} U_{\beta k} \tau_{k k} V_{k a}\right|^{2}, \\
& =\sum_{a}^{N} \sum_{k, k^{\prime}}^{K} U_{\beta k}^{*} U_{\beta k^{\prime}} \tau_{k k} \tau_{k^{\prime} k^{\prime}} V_{k a}^{*} V_{k^{\prime} a} .
\end{aligned}
$$

We use the unitarity of $V$ to get rid of the sum over $a$

$$
\widetilde{I}_{\beta}=\sum_{k}^{K}\left|U_{\beta k}\right|^{2} \tau_{k k}^{2}
$$

Our model allows us to average over $U$ and $\tau$ separately to find

$$
\left\langle\widetilde{I}_{\beta}\right\rangle=\left\langle\tau^{2}\right\rangle \frac{K}{M}
$$

In the situation where $M=K=N$, the fraction of the light that is transmitted into channel $\beta$ is equal to the total transmission before optimization (Eq. (8.11)).

\section{Total transmission}

We now calculate the transmitted field for the optimized incident wavefront given by Eq. (8.14). Using Eq. (8.6) we find

$$
\widetilde{E}_{b}=\sum_{a}^{N}\left(\sum_{k}^{K} U_{b k} \tau_{k k} V_{k a}\right) A_{0}\left(\sum_{k^{\prime}}^{K} U_{\beta k} \tau_{k^{\prime} k^{\prime}} V_{k^{\prime} a}\right)^{*} .
$$

Unitarity of $V$ implies that $\sum_{a}^{N} V_{k a} V_{k^{\prime} a}^{*}=\delta_{k k^{\prime}}$. We now have

$$
\widetilde{E}_{b}=A_{0} \sum_{k}^{K} U_{b k} U_{\beta k}^{*} \tau_{k k}^{2}
$$

The total transmission after optimization now follows from Eq. (8.7),

$$
\widetilde{T}_{\text {tot }}=A_{0}^{2} \sum_{b}^{M}\left(\sum_{k}^{K} U_{b k} U_{\beta k}^{*} \tau_{k k}^{2}\right)\left(\sum_{k^{\prime}}^{K} U_{b k^{\prime}}^{*} U_{\beta k^{\prime}} \tau_{k^{\prime} k^{\prime}}^{2}\right) .
$$

Using unitarity of $U$ in the summation over $b$ we find

$$
\widetilde{T}_{\text {tot }}=A_{0}^{2} \sum_{k}^{K}\left|U_{\beta k}\right|^{2} \tau_{k k}^{4} .
$$


The prefactor $A_{0}$ was defined to normalize the incident wavefront. Using Eq. (8.14) we find

$$
A_{0}^{-2}=\sum_{a}^{N}\left|t_{\beta a}\right|^{2}=\sum_{k}^{K}\left|U_{\beta k}\right|^{2} \tau_{k k}^{2},
$$

where the equality of Eqs. (8.15) and (8.18) was used in the last step. Finally, we have

$$
\widetilde{T}_{\text {tot }}=\frac{\sum_{k}^{K}\left|U_{\beta k}\right|^{2} \tau_{k k}^{4}}{\sum_{k^{\prime}}^{K}\left|U_{\beta k^{\prime}}\right|^{2} \tau_{k^{\prime} k^{\prime}}^{2}} .
$$

Here we see that the numerator is a weighted average over $\tau^{4}$ and the denominator is a weighted average over $\tau^{2}$ with equal weights. Averaging over $U$ gives

$$
\left\langle\widetilde{T}_{\text {tot }}\right\rangle=\frac{\left\langle\tau^{4}\right\rangle}{\left\langle\tau^{2}\right\rangle} \equiv C_{4,2} .
$$

We see that the total transmission after optimizing the incident wavefront is equal to $C_{4,2}$, which is defined as the ratio between the fourth and the second moment of $\tau$. $C_{4,2}$ does not depend on the refractive indices outside the sample. Using the distribution $p(T)$ that is predicted by RMT (Eq. (8.4)), $C_{4,2}$ can be calculated

$$
C_{4,2}=\frac{2}{3}
$$

Surprisingly, $C_{4,2}$ turns out to be a universal constant. A fraction of $2 / 3$ of the power of an ideally optimized wavefront is transmitted, regardless of the thickness of the sample.

\subsubsection{Comparison with the uncorrelated model}

The RMT model is very similar to the uncorrelated transmission coefficients (UTC) model in the sense that both models use a matrix with statistically equivalent random elements. The singular value decomposition that formed the start of our RMT calculations can also be performed on the UTC matrix and all observables can be calculated in exactly the same way. In effect, the only difference between the two models is the distribution of transmission eigenvalues. When we choose $\left\langle\tau^{2}\right\rangle$ to be equal in both models, the only observable difference is the value of $C_{4,2}$.

RMT takes into account the correlations arising from the constraint of energy conservation and gives the universal result of $C_{4,2}=2 / 3$. In the UTC model the matrix elements are uncorrelated. For such a matrix the eigenvalues are expected to have an exponential distribution.[33] In that case, $C_{4,2}=2\left\langle\tau^{2}\right\rangle$. The power in the optimized channel increases to $\left\langle\tau^{2}\right\rangle$, whereas the background transmission remains constant at $\left\langle\tau^{2}\right\rangle$. The optimized transmission is twice the original transmission and, therefore, it is not a universal constant in this model.

Apparently, $C_{4,2}$ is an accessible observable that depends on the symmetry of the disordered system, which makes it very interesting to determine it experimentally. 


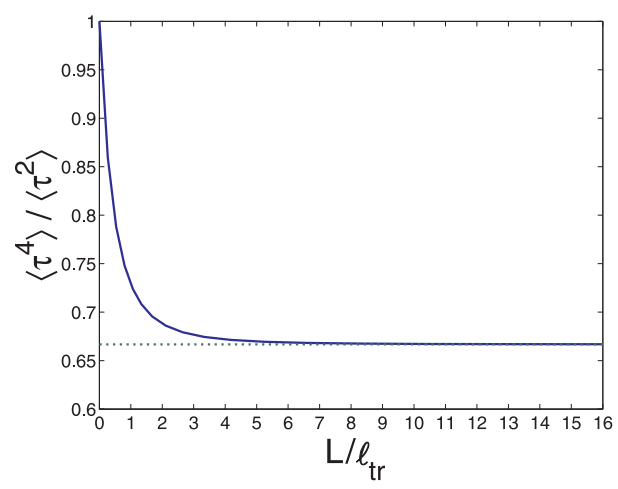

Figure 8.8: Ratio of the fourth moment to the second moment of the transmission eigenvalues $\left(C_{4,2}\right)$ as a function of sample thickness. With increasing thickness, $C_{4,2}$ rapidly converges to $2 / 3$.[2]

\subsubsection{Random matrix theory for thin samples}

We calculated $C_{4,2}=2 / 3$ using the RMT eigenvalue distribution of Eq. (8.4). This equation is only valid in the limit of a 'thick' sample. To investigate when a sample can be considered thick, we use the equation for the exact probability distribution of transmission eigenvalues as calculated by Beenakker[2]. The exact equation is more involved than its limit in the diffusion regime. Beenakker defines $x \equiv \operatorname{arccosh}(1 / \tau)$ and then derives

$$
P(x, s)=\frac{2}{\pi} \operatorname{Im} U\left(x-i 0^{+}, s\right),
$$

with

$$
s=\frac{4 L}{3 \ell_{t r}}
$$

and $U$ is the solution to the implicit equation

$$
U(\zeta, s)=U_{0}(\zeta-s U(\zeta, s)),
$$

with starting condition $U_{0}(\zeta)=\operatorname{coth}(\zeta)$. We used these equations to numerically evaluate $C_{4,2}$ and we found that $C_{4,2}$ converges to $2 / 3$ with increasing sample thickness (Fig. 8.8). The convergence is very rapid: starting at $C_{4,2}=1$ for an infinitely thin sample, $C_{4,2}$ is already at 0.7 for samples that are only one mean free path thick. Therefore, we conclude that samples in our experimental range can be considered thick and Eq. (8.4) can be used.

\subsubsection{Non-diffusive behavior}

The distribution of eigenvalues depends on the transport physics in the disordered medium. In the previous section, we saw that, for very thin samples, the distribution depends on the thickness of the sample.

In microwave experiments, it was observed that absorption in the medium changes the eigenvalue distribution to an exponential distribution.[34] This result can be understood in the sense that there is no constraint of energy conservation in such sys- 


\begin{tabular}{|l|c|c|}
\hline & passive & active \\
\hline single channel & $\left\langle I_{\beta}\right\rangle=\left\langle\tau^{2}\right\rangle \frac{K}{M_{\text {eff }} N}$ & $\left\langle\widetilde{I}_{\beta}\right\rangle=\left\langle|\gamma|^{2}\right\rangle\left\langle\tau^{2}\right\rangle \frac{K}{M}$ \\
total & $\left\langle T_{\text {tot }}\right\rangle=\left\langle\tau^{2}\right\rangle \frac{K}{N}$ & $\left\langle\widetilde{T}_{\text {tot }}\right\rangle=\left\langle|\gamma|^{2}\right\rangle \frac{2}{3}+\left(1-\left\langle|\gamma|^{2}\right\rangle\right)\left\langle\tau^{2}\right\rangle \frac{K}{N}$ \\
\hline
\end{tabular}

Table 8.2: Value of some important observables in a realistic experimental situation (also compare Table. (8.1)).

tems.[33] Therefore, the elements of the transmission matrix are uncorrelated, just as in the UTC model.

In the limit of extremely strong scattering $(k \ell \approx 1)$ interference effects dramatically change the transport properties of waves. $[35,36]$ This effect is known as Anderson localization. The observation of Anderson localization of light[37-39] has proven very difficult, especially because absorption can, in many situations, have a similar signature[40].

Both absorption and localization make that the extended modes vanish, thereby decreasing $C_{4,2}$. However, in quasi-1D systems the effect of localization on the distribution of eigenvalues is rather spectacular. At the localization threshold, eigenvalues start to cluster. This effect is called eigenvalue crystallization and leads to an oscillating eigenvalue distribution (see e.g. [2]). The study of localization in open 3dimensional systems is an ongoing field of research. Recent results[41] predict the distribution of decay rates of optical modes. It would be very interesting to know the distribution of transmission eigenmodes in such a 3-dimensional system and investigate if, by measuring $C_{4,2}$, the difference between localization and absorption can be established experimentally.

\subsection{Random matrix theory in an optical experimental situation}

In this section, we extend random matrix theory of wave scattering to describe a realistic optical experimental situation. First, the geometrical correlations that are present in a slab geometry are analyzed and then the effect of limitations of the modulator is taken into account. We find that a realistic experimental situation can be described with the degree of amplitude control $\gamma$ that accounts for all deviations from the 'ideal' waveguide geometry. The value of the observables in a realistic situation are summarized in Table 8.2.

\subsubsection{Slab geometry}

In our experiments we have a slab geometry as is depicted in Fig 8.9. In this geometry, not all channels are equivalent. When light is focused on incident channel $a$, transmitted channels directly opposite $a$ will receive more intensity than channels that are further away. To include this geometric effect, we recognize that the average 


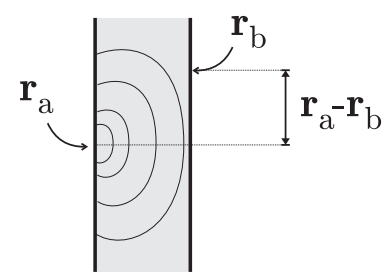

Figure 8.9: Slab geometry. Light diffuses from a point with surface coordinates $\mathbf{r}_{a}$ on the front of the sample to a point $\mathbf{r}_{b}$ at the back of the sample.

transmission coefficient is a function of the relative position of the channels.

$$
\left\langle\left|t_{b a}\right|^{2}\right\rangle=f\left(\left|\mathbf{r}_{b}-\mathbf{r}_{a}\right|\right)\left\langle\tau^{2}\right\rangle \frac{K}{M N},
$$

where all channels are defined in $r$ space. $\mathbf{r}_{a}$ and $\mathbf{r}_{b}$ are the 2-D coordinates of, respectively, the incident and the transmitted channels at the front and back sample surfaces. In a waveguide geometry $f\left(\left|\mathbf{r}_{\beta}-\mathbf{r}_{a}\right|\right)=1$. In a slab geometry $f$ can be calculated using diffusion theory. It is a function that starts at 1 and decreases with distance $\left|\mathbf{r}_{\beta}-\mathbf{r}_{a}\right|$ with a typical decay length that is comparable to the thickness of the sample. Furthermore, $f$ depends on the mean free path and the extrapolation lengths. Since $f$ decreases with distance, channels that are further away from the target contribute less. The effective total number of channels that contribute to the intensity in $\beta$ is given by

$$
N_{\text {eff }}=\sum_{a}^{N} f\left(\left|\mathbf{r}_{\beta}-\mathbf{r}_{a}\right|\right) .
$$

$N_{\text {eff }}$ converges to a finite value in the limit $K, M, N \rightarrow \infty$. In this limit, the waveguide model transforms into a model for an infinite slab and all physical quantities become independent of $N$. Similarly, the effective number of transmitted channels at the back of the sample originating from a point source at $\mathbf{r}_{a^{\prime}}$ equals

$$
M_{\mathrm{eff}}=\sum_{b}^{M} f\left(\left|\mathbf{r}_{b}-\mathbf{r}_{a^{\prime}}\right|\right)=\frac{M}{N} N_{\mathrm{eff}} .
$$

To introduce spatial dependency in the RMT formalism, we use the singular value decomposition (Eq. (8.3)) to find

$$
\left\langle\left|t_{b a}\right|^{2}\right\rangle=\left\langle\left|\sum_{k}^{K} U_{b k} \tau_{k k} V_{k a}\right|^{2}\right\rangle .
$$

Assuming that $\tau$ is still independent of $U$ and $V$, all geometrical effects are accounted for by imposing the following correlation between on $U$ and $V$

$$
\left\langle\left|U_{b k} V_{k a}\right|^{2}\right\rangle=f\left(\left|\mathbf{r}_{b}-\mathbf{r}_{a}\right|\right) \frac{1}{M N}
$$

In the previous sections, we (implicitly) used the assumption that the matrices $U$ and $V$ are uncorrelated only in the calculation of $\left\langle I_{b}\right\rangle$; all other observables in Table 8.1 
are not affected by this correlation. In the active measurements, the incident field is matched to the sample to maximize $I_{b}$. In a passive measurement, however, the incident illumination can have any arbitrary profile that only depends on the experimental configuration. The average value for $I_{b}$ depends on this intensity profile $I_{a}$

$$
\left\langle I_{b}\right\rangle=\sum_{a}^{N} f\left(\left|\mathbf{r}_{b}-\mathbf{r}_{a}\right|\right)\left\langle\tau^{2}\right\rangle \frac{K}{M N} I_{a} .
$$

When the detector is further away from the sample than the diameter of the diffuse spot, it is convenient to define far-field angular channels (far-field speckles). The number of such channels is $M_{\text {eff }}$ (also see Ref. 42 for a discussion on the number of independent channels). In that case, Eq. (8.36) reduces to

$$
\left\langle I_{b}\right\rangle=\left\langle\tau^{2}\right\rangle \frac{K}{M_{\mathrm{eff}} N},
$$

where the total incident power was assumed to be normalized. All other observables that were derived above are not affected by the slab geometry. It is now possible to supplement these results with spatial information. We can, for instance, calculate the intensity profile of the optimal incident field (Eq. (8.14))

$$
\left\langle\left|\widetilde{E}_{a}\right|^{2}\right\rangle=\left\langle A_{0}^{2}\right\rangle\left\langle\left|t_{\beta a}\right|^{2}\right\rangle=\left\langle A_{0}^{2}\right\rangle f\left(\left|\mathbf{r}_{\beta}-\mathbf{r}_{a}\right|\right)\left\langle\tau^{2}\right\rangle \frac{K}{M N}
$$

Below, we will use this intensity profile to determine how well a generated field matches the optimal field.

\subsubsection{Wavefront modulation imperfections}

So far, we have assumed that the incident wavefront is constructed with an infinitely large perfect wavefront generator. All practical imperfections, such as a finite size modulator or phase only modulation, are accounted for by a single parameter $\gamma$. We start by decomposing the incident field into a contribution to the perfect incident field and an orthogonal part $\Delta E_{a}$

$$
E_{a}=\gamma \widetilde{E}_{a}+\sqrt{1-|\gamma|^{2}} \Delta E_{a},
$$

where $\gamma$ is the inner product of the optimal field and the generated field

$$
\gamma \equiv \sum_{a}^{N} \widetilde{E}_{a}^{*} E_{a}
$$

With an ideal modulator, the optimal field can be generated perfectly and $\gamma=1$. All experimental limitations decrease $\gamma$. In the next section, we give some examples how to calculate $\gamma$ in realistic situations. Now, we first calculate how $\gamma$ effects the observables $\widetilde{I}_{\beta}$ and $\widetilde{T}_{\text {tot }}$. Since $\Delta E_{a}$ is orthogonal to the optimal field, it does not contribute to the intensity in the target focus. After optimization, the intensity in the focus is given by (compare Eq. (8.19))

$$
\left\langle\widetilde{I}_{\beta}\right\rangle=\left\langle|\gamma|^{2}\right\rangle\left\langle\tau^{2}\right\rangle \frac{K}{M} .
$$


To calculate the total transmission, we use a similar approach

$$
\left\langle\widetilde{T}_{\text {tot }}\right\rangle=\left\langle|\gamma|^{2}\right\rangle C_{4,2}+\left(1-\left\langle|\gamma|^{2}\right\rangle\right)\left(\left\langle\tau^{2}\right\rangle \frac{K}{N}-\epsilon\right),
$$

where $\left\langle\tau^{2}\right\rangle K / N$ is the transmission coefficient of the unoptimized field and $\epsilon$ is a small correction term takes into account the fact that the total transmission of the orthogonal field is slightly lower than the total transmission of a random field since it does not include the contribution of transmitted channel $\beta$. Also, at the back of the sample the transmitted fields corresponding to $\Delta E$ and $\widetilde{E}_{a}$ are not completely orthogonal. Both effects are of order $1 / N_{\text {eff }}$ and partially cancel. Therefore it is safe to neglect $\epsilon$.

When the incident field is not shaped to fit the scattering matrix of the sample, there will still be some accidental overlap with the optimal field. For such an incident field, $\left\langle|\gamma|^{2}\right\rangle$ is of order $1 / N_{\text {eff }}$ and will be neglected.

\subsubsection{Examples of realistic experimental situations}

We now calculate $\left\langle|\gamma|^{2}\right\rangle$ for various experimental conditions. Eqs. (8.41) and (8.42) can then be used to obtain the intensity in the focus, as well as the total transmission after optimization.

\section{Example 1: Finite size modulator}

Suppose the light modulator illuminates a finite area that contains $N_{s}$ independent scattering channels. The modulator perfectly modulates amplitude and phase of all these scattering channels. Then

$$
\gamma=\sum_{a}^{N_{s}} \widetilde{E}_{a}^{*} E_{a}=A_{0} A_{f} \sum_{a}^{N_{s}}\left|t_{\beta a}\right|^{2},
$$

where $A_{0}$ normalizes the optimal field (see Eq. (8.24)) and $A_{f}$ normalizes the actually generated field. Using Eq. (8.31) to introduce position dependence, we find

$$
\left\langle|\gamma|^{2}\right\rangle=\frac{\sum_{a}^{N_{s}} f\left(\left|\mathbf{r}_{\beta}-\mathbf{r}_{a}\right|\right)}{\sum_{a}^{N} f\left(\left|\mathbf{r}_{\beta}-\mathbf{r}_{a}\right|\right)}=\frac{\sum_{a}^{N_{s}} f\left(\left|\mathbf{r}_{\beta}-\mathbf{r}_{a}\right|\right)}{N_{\text {eff }}} .
$$

In the limit where the light modulator is very large $\gamma \rightarrow 1$. In the limit where the modulator is much smaller than the diffuse spot described by $f$, the degree of control is $\left\langle|\gamma|^{2}\right\rangle=N_{s} / N_{\text {eff. }}$.

\section{Example 2: Limited resolution}

We first calculate $\left\langle|\gamma|^{2}\right\rangle$ for the situation where the light modulator has a limited spatial resolution. We assume that each of the $N_{s}$ segments of the phase modulator illuminates a spatial cluster of $N / N_{s}$ scattering channels. We group the channels by segment and use Eq. (8.40) to find

$$
\gamma=\sum_{s}^{N_{s}} \sum_{a \in\left\{a_{s}\right\}} \widetilde{E}_{a}^{*} E_{s}
$$


where $\left\{a_{s}\right\}$ is the collection of channels that is illuminated by segment $s . E_{s}$ is the field that is generated by segment $s$ of the modulator. The incident field is optimized for a whole cluster at a time. After an optimization we have

$$
\begin{aligned}
& E_{s}=A_{s} \sum_{a \in\left\{a_{s}\right\}} \widetilde{E}_{a} \\
& A_{s} \equiv\left(\frac{N}{N_{s}} \sum_{s}^{N_{s}}\left|\sum_{a \in\left\{a_{s}\right\}} \widetilde{E}_{a}\right|^{2}\right)^{-1 / 2}
\end{aligned}
$$

where the prefactor $A_{s}$ normalizes the total power of the incident field. Substituting Eq. (8.46) in Eq. (8.45) we find

$$
\begin{gathered}
\gamma=A_{s} \sum_{s}^{N_{s}}\left|\sum_{a \in\left\{a_{s}\right\}} \widetilde{E}_{a}\right|^{2}, \\
|\gamma|^{2}=\frac{N_{s}}{N} \sum_{s}^{N_{s}}\left|\sum_{a \in\left\{a_{s}\right\}} \widetilde{E}_{a}\right|^{2} .
\end{gathered}
$$

Because elements of $\widetilde{E}_{a}$ are independent and $\widetilde{E}_{a}$ is normalized, we have

$$
\left\langle\widetilde{E}_{a} \widetilde{E}_{a^{\prime}}\right\rangle=\delta_{a^{\prime} a} \frac{1}{N}
$$

which leads to

$$
\left\langle|\gamma|^{2}\right\rangle=\frac{N_{s}}{N}
$$

\section{Example 3: Phase only modulation}

We consider the case of a phase only modulator that perfectly controls the phase of all $N$ incident waveguide channels. The amplitude of the channels is fixed at $1 / \sqrt{N}$. For this experimental configuration, we find

$$
\begin{gathered}
\gamma=\sum_{a}^{N} \widetilde{E}_{a}^{*} E_{a}=\frac{1}{\sqrt{N}} \sum_{a}^{N}\left|\widetilde{E}_{a}\right|, \\
\gamma^{2}=\frac{1}{N} \sum_{a, a^{\prime} \neq a}^{N}\left|\widetilde{E}_{a}\right|\left|\widetilde{E}_{a^{\prime}}\right|+\frac{1}{N} \sum_{a^{\prime \prime}}\left|\widetilde{E}_{a^{\prime \prime}}\right|^{2} .
\end{gathered}
$$

To average, we use the complex Gaussian probability function of $\widetilde{E}_{a}$ to arrive at

$$
\left\langle\gamma^{2}\right\rangle=\frac{\pi}{4}+\frac{1}{N}\left(1-\frac{\pi}{4}\right) .
$$




\section{Example 4: Realistic experimental conditions}

In this example, we calculate the degree of control under realistic experimental conditions. We analyze the geometry that was used for measuring the data in Fig. 3.4 where the enhancement as a function of the number of modulator segments is plotted. In this geometry, no lenses were used behind the sample; the camera was approximately $30 \mathrm{~cm}$ away from the sample surface. The incident light is focused on the sample surface and illuminates a spot that is much smaller than a millimeter. Analysis of the measured $t_{b a}$ coefficients showed that, on average, all segments contribute approximately equally to the amplitude of the target (variations are within 15\%). Furthermore, limitations of the phase modulator made that the generated field had a bias. On average, $14 \%$ of the incident intensity had a constant phase and could not be shaped.

Since all segments contribute equally, we expect the enhancement to be given by a combination of Example 2 and Example 3. Also taking into account that $14 \%$ of the incident light could not be shaped, we find

$$
\left\langle|\gamma|^{2}\right\rangle=0.86 \frac{N_{s}-1}{N_{\text {eff }}} \frac{\pi}{4}+\frac{1}{N_{\text {eff }}},
$$

which is an upper limit for the case where the sample is absolutely stable and there is no influence of measurement noise. We can now calculate the expected maximum intensity enhancement $\eta$

$$
\eta \equiv \frac{\left\langle\widetilde{I}_{\beta}\right\rangle}{\left\langle I_{\beta}\right\rangle} .
$$

Using Table 8.2 with Eq. (8.55) yields

$$
\begin{aligned}
\eta & =\left\langle|\gamma|^{2}\right\rangle M_{\mathrm{eff}} \frac{N}{M} \\
& =0.68\left(N_{s}-1\right)+1 .
\end{aligned}
$$

This result is the same as was found with the UTC model in Chapter 3. When all segments contribute equally, the UTC model gives the correct results even if the sample is not in a waveguide geometry. The measured enhancement ( $\eta \approx 0.57 N_{s}$, see Fig. 3.4) was slightly lower than the theoretical value because of measurement noise and due to the fact that segments in the center contribute slightly more than segments close to the edge of the phase modulator. This minute effect could be included in $f$ in Equation (8.44), to calculate a value for $\left\langle|\gamma|^{2}\right\rangle$ that is a few percent lower. In the experiments described in Chapter 3 the background intensity did not increase noticeably. This result is explained by the fact that $N_{s} \ll N_{\text {eff }}$ and, therefore, $\left\langle|\gamma|^{2}\right\rangle \ll 1$. In this regime, the total transmission is hardly affected by an optimization (see Eq. (8.42)).

\subsection{Conclusion}

We developed a model for describing the transport of a waves that are actively shaped to focus through a disordered sample. The new model is based on RMT and, therefore, correctly takes into account correlations between scattering channels. 
The concept of 'active' measurements was introduced. These measurements are based on wavefront shaping. One of the most interesting observables in an active measurement is the total transmission of an optimized wavefront. The fraction of transmitted power for such a wavefront turns out to be $2 / 3$, which is universally true regardless of the thickness of the sample. Physical processes such as absorption and Anderson localization change this value, which makes measuring it extremely interesting.

All results were first calculated in an ideal waveguide geometry and then translated to a realistic experimental slab geometry. This two-step calculation process proved to be a very powerful and versatile technique.

Apart from the observables that were calculated in this chapter, there is a multitude of interesting quantities that can be measured with our active measurement strategy. Since most of the theoretical work has been done on ensemble averages rather than on the subset of extended modes, many questions are still unanswered.

\section{Bibliography}

[1] M. L. Mehta, Random matrices and the statistical theory of energy levels (Academic Press, New York, 1967).

[2] C. W. J. Beenakker, Random-matrix theory of quantum transport, Rev. Mod. Phys. 69, 731 (1997).

[3] T. Guhr, A. Müller-Groeling, and H. A. Weidenmüller, Random matrix theories in quantum physics: Common concepts, Phys. Rept. 299, 189 (1998).

[4] E. P. Wigner, On the statistical distribution of the widths and spacings of nuclear resonance levels, Proc. Cambridge Philo. Soc. 47, 790 (1951).

[5] O. N. Dorokhov, Coexistence of localized and extended electronic states in the metallic phase, Sol. St. Commun. 51, 381 (1984).

[6] P. A. Mello, P. Pereyra, and N. Kumar, Macroscopic approach to multichannel disordered conductors, Ann. Physics 181, 290 (1988).

[7] E. Akkermans and G. Montambaux, Mesoscopic physics of electrons and photons, 2 ed. (Cambridge University Press, Cambridge, 2007).

[8] S. Datta, Electronic transport in mesoscopic systems (Cambridge University Press, Cambridge, 2007).

[9] R. L. Weaver, Spectral statistics in elastodynamics, J. Acoust. Soc. Am. 85, 1005 (1989).

[10] M. R. Schröder, Die Statistischen Parameter der Frequenzkurven von grossen Raumen, Acustika 4, 594 (1954), as discussed in Ref. [3]. English translation in J. Audio Eng. Soc. 35 (1987) 307.

[11] H.-J. Stöckmann and J. Stein, "Quantum" chaos in billiards studied by microwave absorption, Phys. Rev. Lett. 64, 2215 (1990).

[12] E. Doron, U. Smilansky, and A. Frenkel, Experimental demonstration of chaotic scattering of microwaves, Phys. Rev. Lett. 65, 3072 (1990).

[13] P. W. Brouwer and M. Büttiker, Charge-relaxation and dwell time in the fluctuating admittance of a chaotic cavity, Europhys. Lett. 37, 441 (1997).

[14] B. A. van Tiggelen, P. Sebbah, M. Stoytchev, and A. Z. Genack, Delay-time statistics for diffuse waves, Phys. Rev. E 59, 7166 (1999).

[15] H. Schomerus, K. J. H. van Bemmel, and C. W. J. Beenakker, Localization-induced coherent backscattering effect in wave dynamics, Phys. Rev. E 63, 26605 (2001). 
[16] S. E. Skipetrov and B. A. van Tiggelen, Dynamics of weakly localized waves, Phys. Rev. Lett. 92, 113901 (2004).

[17] K. L. van der Molen, R. W. Tjerkstra, A. P. Mosk, and A. Lagendijk, Spatial extent of random laser modes, Phys. Rev. Lett. 98, 132901 (2007).

[18] J. B. Pendry, A. MacKinnon, and A. B. Pretre, Maximal fluctuations - a new phenomenon in disordered systems, Physica A 168, 400 (1990).

[19] J. B. Pendry, A. MacKinnon, and P. J. Roberts, Universality classes and fluctuations in disordered systems, Proc. R. Soc. Lond. A 437, 67 (1992).

[20] A. Stone, P. A. Mello, K. A. Muttalib, and J.-L. Picha, in Mesoscopic phenomena in solids, Vol. 30 of Modern Problems in Condensed Matter Sciences, edited by B. L. Altshuler, P. A. Lee, and R. A. Webb (Elsevier Science, New York, 1991), p. 369.

[21] Y. V. Nazarov, Limits of universality in disordered conductors, Phys. Rev. Lett. 73, 134 (1994).

[22] M. C. W. van Rossum and T. M. Nieuwenhuizen, Multiple scattering of classical waves, Rev. Mod. Phys. 71, 313 (1999).

[23] A. Lagendijk, R. Vreeker, and P. de Vries, Influence of internal reflection on diffusive transport in strongly scattering media, Phys. Lett. A 136, 81 (1989).

[24] J. X. Zhu, D. J. Pine, and D. A. Weitz, Internal reflection of diffusive light in random media, Phys. Rev. A 44, 3948 (1991).

[25] M. U. Vera and D. J. Durian, Angular distribution of diffusely transmitted light, Phys. Rev. E 53, 3215 (1996).

[26] M. Shapiro and P. Brumer, Laser control of product quantum state populations in unimolecular reactions, J. Chem. Phys. 84, 4103 (1986).

[27] R. M. Bowman, M. Dantus, and A. H. Zewail, Femtosecond multiphoton dynamics of higher-energy potentials, Chem. Phys. Lett. 174, 546 (1990).

[28] R. S. Judson and H. Rabitz, Teaching lasers to control molecules, Phys. Rev. Lett. 68, 1500 (1992).

[29] D. Zeidler, S. Frey, K.-L. Kompa, and M. Motzkus, Evolutionary algorithms and their application to optimal control studies, Phys. Rev. A 64, 023420 (2001).

[30] T. C. Weinacht and P. H. Bucksbaum, Using feedback for coherent control of quantum systems, J. Opt. B 4, R35 (2002).

[31] J. L. Herek, W. Wohlleben, R. J. Cogdell, D. Zeidler, and M. Motzkus, Quantum control of energy flow in light harvesting, Nature 417, 533 (2002).

[32] S. Mujumdar, M. Ricci, R. Torre, and D. S. Wiersma, Amplified extended modes in random lasers, Phys. Rev. Lett. 93, 053903 (2004).

[33] E. Kogan, P. A. Mello, and H. Liqun, Wave scattering through classically chaotic cavities in the presence of absorption: An information-theoretic model, Phys. Rev. E 61, R17 (2000).

[34] R. A. Méndez-Sánchez, U. Kuhl, M. Barth, C. H. Lewenkopf, and H.-J. Stöckmann, Distribution of reflection coefficients in absorbing chaotic microwave cavities, Phys. Rev. Lett. 91, 174102 (2003).

[35] P. W. Anderson, Absence of diffusion in certain random lattices, Phys. Rev. 109, 1492 (1953).

[36] A. F. Ioffe and A. R. Regel, Non-crystalline, amorphous and liquid electronic smiconductors, Progress in semiconductors 4, 237 (1960).

[37] D. S. Wiersma, P. Bartolini, A. Lagendijk, and R. Righini, Localization of light in a disordered medium, Nature 390, 671 (1997).

[38] F. J. P. Schuurmans, M. Megens, D. Vanmaekelbergh, and A. Lagendijk, Light scattering near the localization transition in macroporous GaP networks, Phys. Rev. Lett. 83, 2183 (1999).

[39] M. Störzer, P. Gross, C. M. Aegerter, and G. Maret, Observation of the critical regime near Anderson localization of light, Phys. Rev. Lett. 96, 063904 (2006).

[40] F. Scheffold, R. Lenke, R. Tweer, and G. Maret, Localization or classical diffusion of light?, 


\section{4 | Bibliography}

Nature 398, 206 (1999).

[41] S. E. Skipetrov and B. A. van Tiggelen, Dynamics of Anderson localization in open 3D media, Phys. Rev. Lett. 96, 043902 (2006).

[42] J. F. de Boer, Optical fluctuations on the transmission and reflection of mesoscopic systems, Ph.D. thesis, University of Amsterdam, Amsterdam, 1995, available on http://cops.tnw.utwente.nl. 


\section{Chapter 9}

\section{Observation of open transport channels in disordered optical systems}

When a beam of light impinges on a strongly scattering object, such as a layer of white paint, tiny particles randomly scatter light in all directions. After a few scattering events, light is completely diffuse. The transport of multiply scattered light is, in general, described very well by a diffusion equation. However, the diffusion equation does not take into account interference effects. The inclusion of interference in the description of disordered wave propagation has led to the discovery of fundamental effects like enhanced backscattering $[1,2]$, universal conductance fluctuations $[3,4]$ and Anderson localization of light[5-7].

Here we demonstrate experimentally a new interference effect that was first predicted by Dorokhov[8]. Like the interference effects mentioned above, this effect was first derived for the propagation of electron wave functions in a disordered waveguide (a metal wire) and then translated to optics. [9, 10] A disordered waveguide can be described as a collection of orthogonal transport channels that each carry a fraction of the incident wave. Using random matrix theory (RMT), Dorokhov showed that due to interference these channels are either open (transmission close to 1) or closed (transmission close to 0 ).

The existence of open transport channels means that it is, in principle, possible to construct a wave that is fully transmitted through a normally opaque mesoscopic object, regardless of the thickness of that object. In a mesoscopic electronic conductor such control over the incident wave is not possible. For light, however, we can shape the wavefront of the incident wave with a high accuracy. In this chapter we show that the shaped wave selectively couples to open transport channels, causing the transmission through an opaque, strongly scattering sample to increase dramatically.

In Section 9.1 we give the theoretical relation for the total transmission of a shaped wavefront. Then, in Section 9.2, the wavefront optimization setup is described. The experimental results are presented in Section 9.3, followed by a more detailed description of the data analysis procedure and an estimation of the experimental error margins in Section 9.4.

\subsection{Expected total transmission}

The average transport of light is described accurately by a diffusion equation. On average, the total transmission coefficient is given by $\left(\ell+z_{e 1}\right) /\left(L+z_{e 1}+z_{e 2}\right)$, with $\ell$ the mean free path for light, $L$ the thickness of the scattering sample and $z_{e 1}$ and $z_{e 2}$ 
extrapolation lengths that account for reflection at the sample interface[11, 12].

To increase the total transmission above the value predicted by diffusion theory, we use a feedback algorithm that shapes the incident wavefront. The algorithm maximizes the intensity in a target focus behind the sample. Since only open transport channels contribute to the intensity in the target, the ideally shaped wavefront selectively couples to these open channels. In the extreme case where all open channels have a transmission coefficient of exactly 1 (the so called maximum fluctuations theorem[13, 14]), light with an optimal wavefront is completely transmitted.

Random matrix theory[8] predicts a distribution that is close to the extreme situation of maximal fluctuations (see Eq. (8.4)). We express the amount of fluctuations in the distribution as $C_{4,2}$, which is defined in Eq. (8.26) and denotes the transmission coefficient of an ideally shaped wavefront. For Dorokhov's distribution $C_{4,2}=2 / 3$. This universal value is independent of the thickness or the mean free path of the sample.

To couple to open channels, a high degree of control over the incident wavefront is essential. When only a small subset of the scattering channels is controlled, the transmission matrix elements are effectively uncorrelated and no open channels can be found. Therefore, the experiment is designed to approximate the ideal wavefront as closely as possible. Still, in any experiment there will be a difference between the optimal wavefront and the field that is actually generated. This is especially true in an open system where the optimal wavefront has infinite support. The total transmission of an incompletely optimized wavefront equals (see Eq. (8.42))

$$
\widetilde{T}_{\text {tot }} \equiv \frac{\widetilde{P}_{\text {tot }}}{P_{\text {in }}}=|\gamma|^{2} C_{4,2}+\left(1-|\gamma|^{2}\right) T_{\text {tot }} \text {, }
$$

where $\widetilde{P}_{\text {tot }}$ is the totally transmitted power of the incompletely optimized wavefront, $P_{\text {in }}$ is the incident power and $T_{\text {tot }} \equiv P_{\text {tot }} / P_{\text {in }}$, with $P_{\text {tot }}$ the totally transmitted power of an non-optimized wavefront. $|\gamma|^{2}$ is the degree of control as defined in Eq. (8.40). In the case that we have full control over the incident wavefront $|\gamma|^{2} \rightarrow 1$ and $\widetilde{T}_{\text {tot }} \rightarrow C_{4,2}$.

The degree of control can be determined experimentally by measuring the power in the target focus $\widetilde{P}_{\beta}$ after optimization. From Eq. (8.41) we can make $|\gamma|^{2}$ explicit

$$
|\gamma|^{2}=\frac{\widetilde{P}_{\beta}}{P_{\text {tot }}} \frac{n_{2}^{2}}{n_{1}^{2}}
$$

where $n_{1}=1$ and $n_{2}=1.52$ are the refractive indices of the medium in front of the sample (air) and behind the sample (glass substrate) respectively. Since $|\gamma|^{2}$ can be determined experimentally, Eq. (9.1) can be solved to find $C_{4,2}$.

\subsection{Experiment}

The experimental apparatus is designed to have optimal control over the incident wavefront. The setup is shown in Fig. 9.1. An expanded beam from a $632.8 \mathrm{~nm}$ HeNe laser is rotated to a $45^{\circ}$ linear polarization by a half waveplate and impinges on a polarizing beam splitter cube. Both horizontal and vertical polarization are modulated using the macropixel modulation method that was described in Section 2.1.4. 


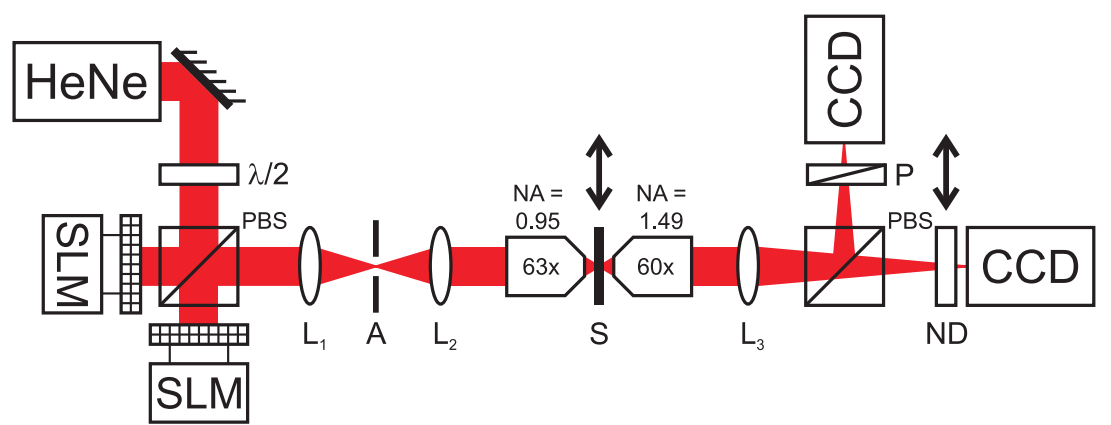

Figure 9.1: Experimental setup for finding open transport channels. HeNe, expanded $632.8 \mathrm{~nm} \mathrm{HeNe}$ laser; $\lambda / 2$, half waveplate; PBS, polarizing beam splitter cube; SLM, spatial light modulator. A, iris diaphragm; 63x, microscope objective; 60x, oil immersion microscope objective; $S$, sample; P, polarizer; ND, neutral density filter; $L_{1}, L_{2}$, $L_{3}$, lenses with focal length of respectively $250 \mathrm{~mm}, 200 \mathrm{~mm}$ and $600 \mathrm{~mm}$. ND and S are translated by computer controlled stages.

The synthesized wavefront is focused onto the sample with a high numerical aperture (NA) objective ${ }^{1}$ so that almost all angular channels are controlled. A high NA oil-immersion objective ${ }^{2}$ collects almost all transmitted light. The immersion oil matches the refractive index of the substrate $n=1.52$ and, therefore, eliminates reflections at the back surface of the substrate. The transmitted light is split into horizontal and vertical polarizations by a beam splitter cube. A second polarizer improves the cube's limited extinction ratio for reflected light. A camera measures the power of the horizontally polarized light in an area with the size of a single speckle to provide feedback for the optimization algorithm. We use the stepwise sequential algorithm (see Section 7.1.1) to optimize the wavefront. A calibrated neutral density filter with a transmission of $1.4 \cdot 10^{-3}$ is automatically placed in front of the camera to be able to measure the high intensity in the target focus after optimization. The magnification of the detection system is $225 \times$. At this magnification, individual speckles are well separated on the camera. A second camera measures the transmission in the vertical polarization channel. This camera is not used during the optimization procedure itself.

The sample is a layer of spray-painted $\mathrm{ZnO}$ particles (see Section 2.4) in an air matrix. The particles have an average diameter of $200 \mathrm{~nm}$, which makes them strongly scattering for visible light. The mean free path was determined by measuring the total transmission and equals $0.85 \pm 0.15 \mu \mathrm{m}$ at a wavelength of $632.8 \mathrm{~nm}$. Two samples were used: one with a thickness of $5.7 \pm 0.5 \mu \mathrm{m}$, and one that is approximately twice as thick $(11.3 \pm 0.5 \mu \mathrm{m})$. The sample was mounted on a motorized translation stage that translates the sample perpendicular to the axis of the objectives between experiments. This way, a different random configuration of scatterers could be used for each experiment. The translation stage also provides a reliable way of making reference measurements (see Section 9.3).

\footnotetext{
${ }^{1}$ Zeiss 440068-0000-000 Achroplan 63x/NA $=0.95$ without cover glass correction.

${ }^{2}$ Nikon MRD01691 CFI Plan Apochromat TIRF 60x/NA = 1.49
} 

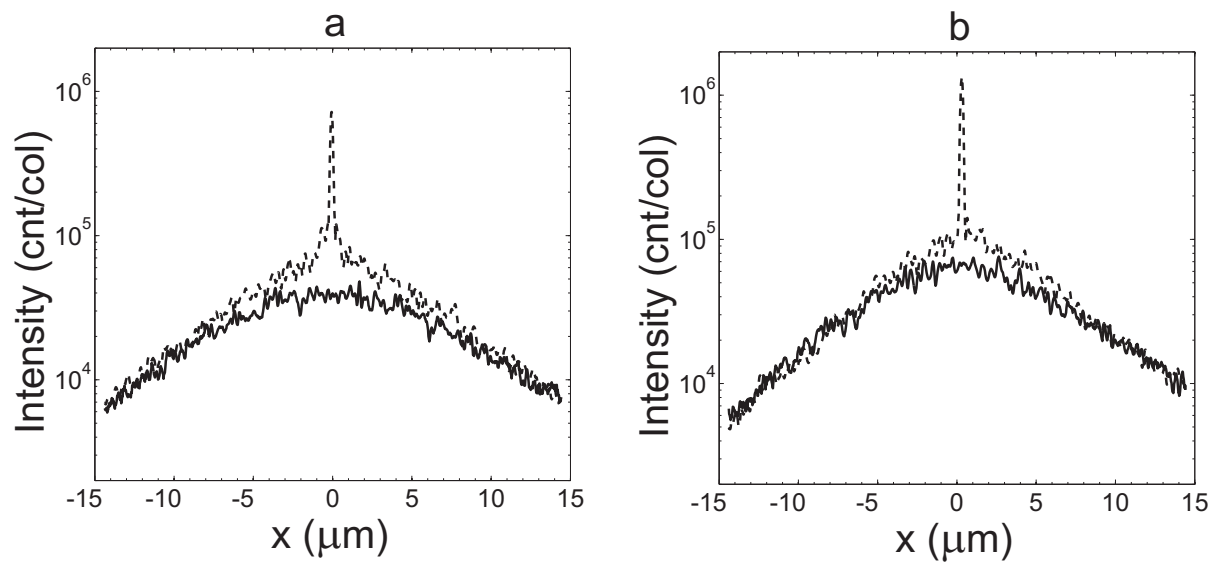

Figure 9.2: Logarithmic plot of the intensity transmission in the horizontal polarization channel. The intensity is integrated per column. a) $11.3 \mu \mathrm{m}$-thick layer of $\mathrm{ZnO}$ pigment. Solid curve, transmission of non-optimized wavefront; Dashed curve, transmission of optimized wavefront. Measurements with and without neutral density filter were combined to cover the full dynamic range. b) Same for a $5.7 \mu$ m-thick layer of $\mathrm{ZnO}$ pigment.

\subsection{Results}

Optimizing the incident wavefront caused the intensity in the target to increase dramatically. The increase in the target intensity was a factor of $746 \pm 28$ for the $11.3 \mu \mathrm{m}$ thick sample and a factor of $671 \pm 14$ for the $5.7 \mu \mathrm{m}$-thick sample in the particular cases that are shown in Fig. 9.2. In addition, the intensity also increased outside the target area, even though the algorithm only used the intensity in the target as feedback. This is an important observation since it indicates that the transmission matrix is correlated. By optimizing transmission to a single target, the incident intensity is redistributed from closed channels to open transport channels. If the elements of the transmission matrix had been uncorrelated, the background intensity would not have changed (also see Section 8.2.3).

The increase in the background intensity is limited to an area of a few micrometers around the target. The diameter of this area seems to be roughly proportional to the thickness of the sample. This makes sense as, in a slab geometry, propagation paths that are further apart than the thickness of the sample are essentially uncorrelated. The intensity increase of the background is maximal just around the optimized spot. There, the intensity increased by a factor of $4.1 \pm 0.7$ and $2.3 \pm 0.3$ for the thick and the thin sample respectively ${ }^{3}$. The total transmission in the background (not including the optimized spot) increased a factor of $1.32 \pm 0.05$ and $1.26 \pm 0.08$, respectively.

The algorithm optimizes the transmission through the whole optical system that is between the light modulator and the camera. To make sure that the extra intensity is indeed the result of an increased transmissivity of the sample, we need to exclude the trivial effect of an increased transmission trough e.g. the microscope objective.

\footnotetext{
${ }^{3}$ In Fig. 9.2 the intensity increase appears to be lower since the figure shows the intensity integrated over all columns.
} 


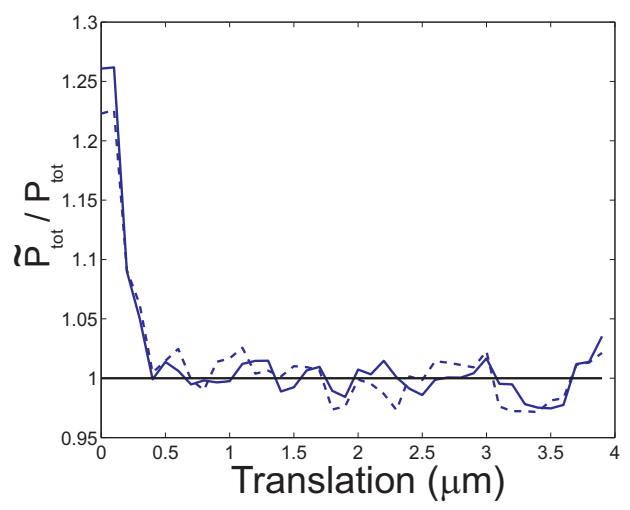

Figure 9.3: Typical total transmission after optimization as a function of sample displacement. Solid curve, horizontal polarization. Dashed curve, vertical polarization. Curves are normalized to the reference transmission (average intensity between $0.6 \mu \mathrm{m}$ and $3.9 \mu \mathrm{m}$ displacement). Fluctuations of the intensity are the result of speckle.

Therefore, we used exactly the same wavefront for measuring the non-optimized reference transmission as we used to measure the optimized transmission. The only difference between the two measurements is a slight translation of the sample. At the new position, the scattering behavior of the sample is different and the optimized wavefront has no special significance anymore.

The effect of translating the $11.3 \mu \mathrm{m}$-thick sample is shown in Fig. 9.3. Initially, the total transmission in both of the polarization channels has increased significantly. The intensity on one of the cameras is slightly higher since it includes the contribution of the optimized spot. Since the pixels in the target area saturate, in this plot the difference is relatively small. After translating the sample over $600 \mathrm{~nm}$, the effect of optimizing the wavefront is completely lost. The transmission is averaged over $34 \mathrm{im}$ ages taken at displacements increasing from $0.6 \mu \mathrm{m}$ to $3.9 \mu \mathrm{m}$ to obtain the reference transmitted power for the non-optimized situation $\left(P_{\text {tot }}\right)$. We observe a slight variation in the total transmission when the sample is translated. The expected variance of fluctuations in the total transmission is $1 /(2 g)$ (see e.g. Ref. 15), where $g \equiv N T_{\text {tot }}$, with $N$ the number of participating scattering channels. From the size of the diffuse spot we estimate $N \approx 4.5 \cdot 10^{3}$. With a measured transmission of $T_{\text {tot }}=0.24$ (see Section 9.4), we expect fluctuations with a standard deviation of $2 \%$, which is consistent with the data.

We performed automated sequences of optimizations. Between each measurement, the sample was translated to find a new realization of disorder. For each of the measurements, we determined the degree of control using Eq. (9.2). In Fig. 9.4 we plotted the increase in total transmission versus the degree of control for a $11.3 \mu \mathrm{m}$ thick sample. The total transmission increases linearly with $|\gamma|^{2}$. The maximum increase in transmission that was achieved is $40 \%$. We conclude that, with increasing degree of control, a larger fraction of the incident intensity couples to open channels, causing the total transmission to increase.

From a linear regression we find that the curve has a slope of 1.7. With an original 


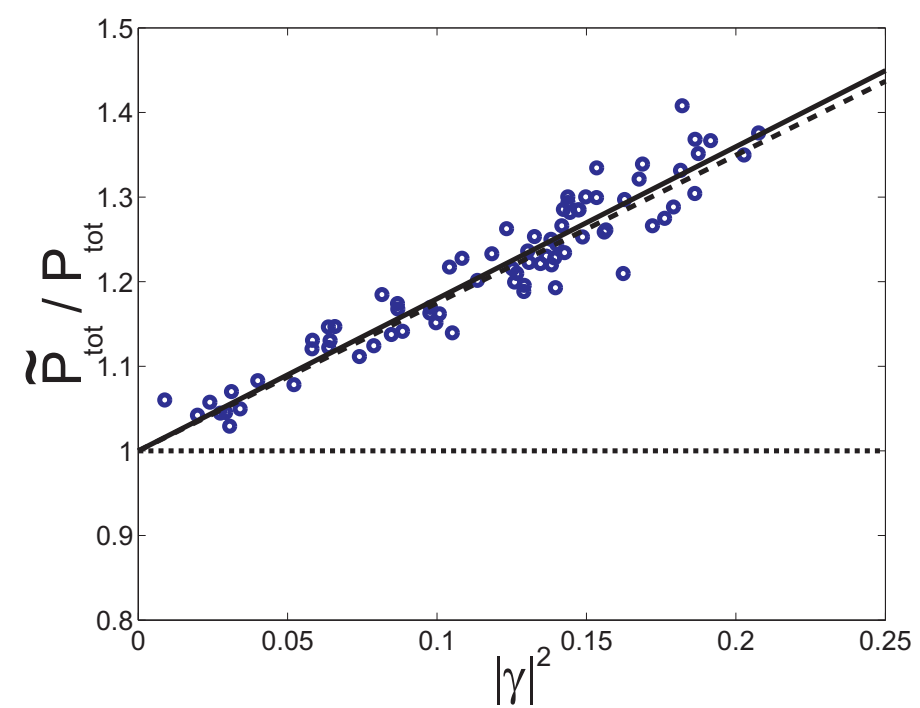

Figure 9.4: Normalized total transmission as a function of the degree of control $|\gamma|^{2}$ for a single sequence of measurements. Circles, measured transmission after constructing the incident wavefront; solid line, linear regression to the measurements; dotted line, diffusion theory; dashed line, random matrix theory, $C_{4,2}=2 / 3$ (there are no adjustable parameters).

average transmission coefficient of $T_{\text {tot }}=0.24$ we expect a total transmission of 0.65 when $|\gamma|^{2}=1$. This result is very close to the expected value of $2 / 3$.

For this particular sequence, we reached a maximum control fraction of $|\gamma|^{2}=0.21$. The algorithm used 3816 control segments and phase only modulation. In this situation $|\gamma|^{2}$ can theoretically not be higher than $(3816 / N)(\pi / 4) \approx 0.67$. Even in a perfectly stable system, $|\gamma|^{2}$ will be lower due to geometrical effects (see Section 8.3.2), so $|\gamma|^{2}=0.21$ is in line with expectations. The variations in $|\gamma|^{2}$ are probably the result of drifting environmental conditions that affect the stability of the setup. Although this drift is undesired, it has the advantage of giving a wide range of $|\gamma|^{2}$ to investigate.

We now combine the results from different measurements into a universal plot. To do this, we subtracted the contribution of the uncontrolled fraction of the incident wavefront to find the controlled part of the transmission

$$
T_{\mathrm{c}} \equiv \widetilde{T}_{\mathrm{tot}}-\left(1-|\gamma|^{2}\right) T_{\mathrm{tot}},
$$

From Eq. (9.1) we expect that $T_{\mathrm{C}}=|\gamma|^{2} C_{4,2}$. As a result, all measurements should follow a universal curve with a slope of $C_{4,2}$. In Fig. 9.5 we plotted the controlled transmission for seven measurement sequences performed on two different samples. All data points collapse to a single line, regardless of the thickness of the sample. This is a clear indication that the transmission of the ideal wavefront $\left(C_{4,2}\right)$ is a universal parameter.

For the $11.3 \mu \mathrm{m}$-thick sample the linear regression gives a slope $0.68 \pm 0.01$ and for the $5.7 \mu \mathrm{m}$-thick sample we find a slope of $0.68 \pm 0.02$, where the uncertainties represent the $0.95 \%$ confidence interval of the regression. Combining the data from 


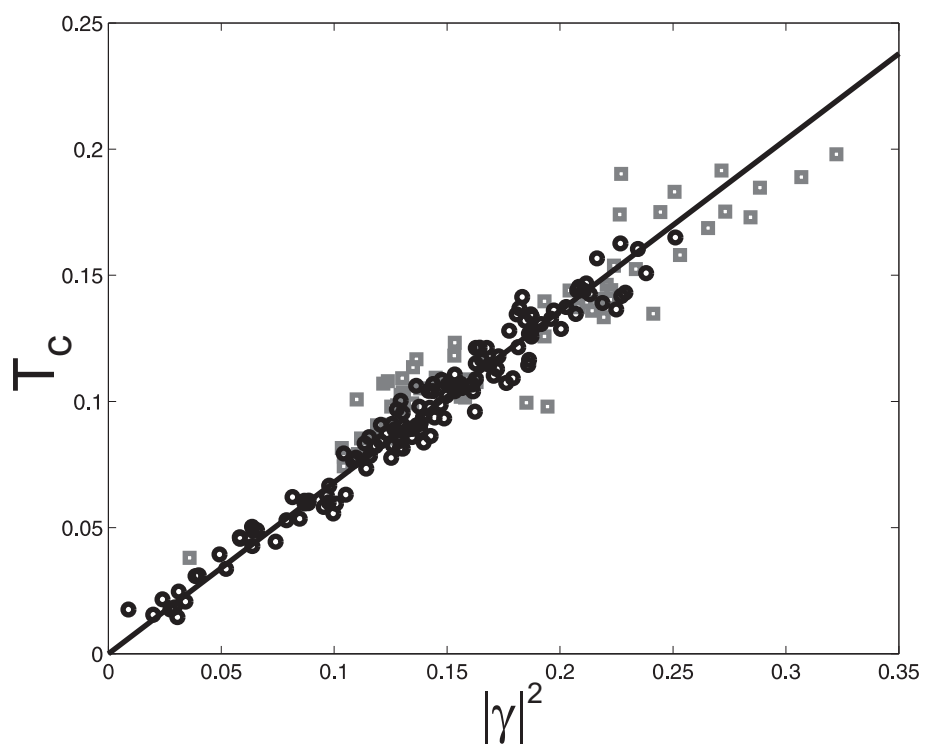

Figure 9.5: Total transmission of the controlled fraction of the wavefront. Data from seven measurement sequences on two different samples collapses to a universal curve. There are no free parameters in the data or the theory. Circles, results for $11.3 \mu \mathrm{m}$-thick sample; squares, results for $5.7 \mu \mathrm{m}$-thick sample; solid line, linear regression with slope of 0.68 . The theoretical curve for $C_{4,2}=2 / 3$ is not shown as it overlaps with the fit.

both samples, we find $C_{4,2}=0.68 \pm 0.07$ where the uncertainty is determined by the worst case systematical errors due to the extrapolation of the intensity profile (see Section 9.4).

\subsection{Details of the data analysis}

In this section we give experimental details about the data analysis. In Section 9.4.1 we explain how the totally transmitted power was measured using a CCD camera. This measurement method introduces a small but significant error in the determination of $C_{4,2}$. Other possible sources of systematical measurement error are discussed in Section 9.4.2. In Section 9.4.3 we explain how the incident intensity was measured accurately.

\subsubsection{Diffuse transmission measurement with a camera}

We measured the totally transmitted power with the CCD cameras behind the sample. The cameras collect most of the transmitted light, but the diffuse spot has such a wide profile that its tails are not imaged onto the CCD chip. Therefore, the camera image is extrapolated to obtain the total intensity.

It turned out to be hard to reliably fit a theoretical curve to the complete intensity 


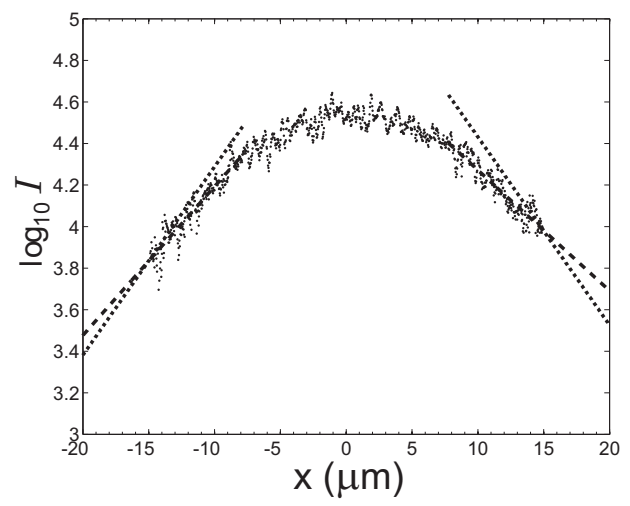

Figure 9.6: Logarithmic plot of the transmitted intensity profile for a reference measurement. The intensity is integrated per column. Dots, measurements; Dashed line, exponential extrapolation; Dotted line, steepest slope that is consistent with diffusion theory.

profile, as the profile depends on the incident wavefront and many other parameters. According to diffusion theory, the transmission profile asymptotically decays exponentially with a decay length of $\left(L+z_{e 1}+z_{e 2}\right) / \sqrt{6}$ (see Section 1.3.4). It, therefore, makes sense to extrapolate the camera image exponentially.

A typical transmission profile of a reference measurement is shown in Fig. 9.6. It can be seen that the exponential extrapolation gives a slight overestimation of the intensity since the measured profile does not fully reach into the exponential asymptotic limit. Alternatively, we extrapolated the profile with the steepest exponential decay that is in agreement with diffusion theory. For this, we took the lower limit for the sample thickness $(L=10.8 \mu \mathrm{m}$ and $L=5.2 \mu \mathrm{m})$, the minimal mean free path $(\ell=0.7)$ and the minimal extrapolation lengths $\left(z_{e 1}=z_{e 2}=2 / 3 \ell\right)$. This alternative extrapolation method (dotted lines in Fig. 9.6) gives a lower limit for the total intensity. By averaging the results of the two methods we find a total intensity of $(2.92 \pm 0.08) \cdot 10^{7}$ counts for the profile in Fig. 9.6, where the error margin is the difference between the results of the two extrapolation methods. A fraction of $82 \%$ of the total intensity fell on the CCD.

The typical uncertainty in this experiment is about $4 \%$. The profiles for the reference intensity $P_{\text {tot }}$ and the optimized intensity $\widetilde{P}_{\text {tot }}$ are almost equal at the edge of the image (see Fig. 9.2). Therefore, it is likely that the error caused by the extrapolation is either positive for both profiles, or negative for both profiles. In this case, the extrapolation only causes an uncertainty of $\pm 2 \%$ in $C_{4,2}$. By calculating $C_{4,2}$ for all combinations of error margins a worst case error margin of $\pm 9 \%$ is found.

\subsubsection{Possible causes of systematical error}

It is possible that small systematical errors are introduced by the imaging system (microscope objective, lenses and camera) that record the transmitted light. We measured the intensity distribution coming from a sample that is illuminated with a wide $(>500 \mu \mathrm{m})$ spot. In this situation, the intensity distribution of the transmitted field 
should be flat (except for speckle). We used this flat-field reference image to compensate for the position dependent sensitivity of the imaging system.

The angular dependency of the imaging system was not calibrated. However, the width of the optimized spot was equal to the diffraction limit of $\lambda /\left(2 n_{2}\right)$ to within an experimental uncertainty of $6 \%$. Therefore, we expect the effect of angular dependency to be below $6 \%$.

\subsubsection{Measurement of the incident power}

With the cameras we measure the total power that is transmitted through a sample before and after an optimization. We also measure $P_{\text {in }}$, which is a reference for the incident power. This reference value is equal to the total power that is incident on the sample times the detection efficiency of the transmitted light. When $P_{\text {in }}$ is known, we can calculate absolute values for the transmission coefficients. In measuring $P_{\text {in }}$, great care was taken not to introduce systematical errors. A complicating factor is that the optimized wavefront is different for every measurement, which causes variations in the power that reaches the sample. To compensate for these variations, we determined $P_{\text {in }}$ using

$$
P_{\text {in }}=P_{\text {tot }} \frac{P_{1}}{P_{2}} \frac{1}{1-R}
$$

where $P_{1}$ is the detected transmitted power with an empty substrate in place of the sample. The substrate was illuminated with an unshaped wavefront: all pixels of the modulator were set to the same phase, so that the incident light forms a focused wave. $P_{2}$ was measured using the same unshaped wavefront, with the sample in place. The sample was at exactly the same position where the optimization was performed. $P_{\text {tot }}$ is the reference transmission of the shaped wavefront. It was measured by translating the sample after each optimization (see Fig. 9.3). The factor $1 /(1-R)$ compensates for the Fresnel reflection at the front surface of the substrate. For a uniformly illuminated microscope objective with NA $=0.95$, the average Fresnel reflection coefficient is $R=$ 0.11 . We determined the transmission coefficient of the unshaped wavefront using $T_{\text {tot }}=(1-R) P_{2} / P_{1}$.

\subsection{Conclusion}

We have found experimental evidence of the existence of open transport channels for light in opaque, strongly scattering materials. A wavefront shaping algorithm was used to focus light through opaque objects. We observed that the transmitted intensity in the target focus increased by almost three orders of magnitude. Simultaneously, the intensity outside the focus increased, even though the algorithm only optimized the intensity in the focus. This result cannot be explained by diffusion theory or by an uncorrelated matrix model. We conclude that the elements of the transmission matrix are correlated as predicted by random matrix theory. Optimizing the transmission at a single point creates a wavefront that selectively couples to open transport channels in the medium.

The total transmission has a linear relation to the intensity in the target focus. This relation confirms the theoretical results from Section 8.3.2. The total transmission 
through a disordered sample was increased by a factor of up to 1.4.

We used the intensity in the target focus to derive the degree of control over the incident wavefront. By considering only the transmission of the controlled fraction of the wavefront, we were able to compare the results for different samples and different realizations of disorder. All results showed a universal behavior that is in excellent quantitative agreement with the distribution of channel transmission coefficients as is predicted by random matrix theory.

\section{Bibliography}

[1] M. P. van Albada and A. Lagendijk, Observation of weak localization of light in a random medium, Phys. Rev. Lett. 55, 2692 (1985).

[2] P. E. Wolf and G. Maret, Weak localization and coherent backscattering of photons in disordered media, Phys. Rev. Lett. 55, 2696 (1985).

[3] J. F. de Boer, M. C. W. van Rossum, M. P. van Albada, T. M. Nieuwenhuizen, and A. Lagendijk, Probability distribution of multiple scattered light measured in total transmission, Phys. Rev. Lett. 73, 2567 (1994).

[4] F. Scheffold and G. Maret, Universal conductance fluctuations of light, Phys. Rev. Lett. 81, 5800 (1998).

[5] D. S. Wiersma, P. Bartolini, A. Lagendijk, and R. Righini, Localization of light in a disordered medium, Nature 390, 671 (1997).

[6] M. Störzer, P. Gross, C. M. Aegerter, and G. Maret, Observation of the critical regime near Anderson localization of light, Phys. Rev. Lett. 96, 063904 (2006).

[7] T. Schwartz, G. Bartal, S. Fishman, and M. Segev, Transport and Anderson localization in disordered two-dimensional photonic lattices, Nature 446, 52 (2007).

[8] O. N. Dorokhov, Coexistence of localized and extended electronic states in the metallic phase, Sol. St. Commun. 51, 381 (1984).

[9] M. C. W. van Rossum, Mesoscopic phenomena in multiple light scattering, Ph.D. thesis, Univeristy of Amsterdam, Amsterdam, 1995, e-print arXiv:cond-mat/9504101.

[10] C. W. J. Beenakker, Random-matrix theory of quantum transport, Rev. Mod. Phys. 69, 731 (1997).

[11] A. Lagendijk, R. Vreeker, and P. de Vries, Influence of internal reflection on diffusive transport in strongly scattering media, Phys. Lett. A 136, 81 (1989).

[12] J. X. Zhu, D. J. Pine, and D. A. Weitz, Internal reflection of diffusive light in random media, Phys. Rev. A 44, 3948 (1991).

[13] J. B. Pendry, A. MacKinnon, and A. B. Pretre, Maximal fluctuations - a new phenomenon in disordered systems, Physica A 168, 400 (1990).

[14] J. B. Pendry, A. MacKinnon, and P. J. Roberts, Universality classes and fluctuations in disordered systems, Proc. R. Soc. Lond. A 437, 67 (1992).

[15] J. F. de Boer, Optical fluctuations on the transmission and reflection of mesoscopic systems, Ph.D. thesis, University of Amsterdam, Amsterdam, 1995, available on http://cops.tnw.utwente.nl. 


\section{Chapter 10}

\section{Summary and outlook}

In this thesis, we explored the use of wavefront shaping to steer light through strongly scattering materials. We found that scattering does not irreversibly scramble the incident wave. By shaping the incident wavefront, an opaque object can form any desired wavefront and focus light as sharply as an aberration free lens. We used feedback from a target behind, or in, an opaque object to shape the incident wave. This way, light was focused through, or inside, an opaque object for the first time ever.

Wavefront shaping provides a new way to perform experiments on strongly scattering samples. By studying the total intensity transmission of a shaped wavefront, we found experimental evidence of open transport channels for light. This is an example of how wavefront shaping can be used to study fundamental properties of scattered waves.

Our results lay both an experimental and a theoretical basis for wavefront shaping experiments. The concepts that were developed are generally applicable to any linear complex system. Therefore, our methods can be directly translated to other disordered systems such as scattering of microwaves, sound, seismic waves, electrons or neutrons. Even though we have only taken some first steps in this field, the outline of future applications and exciting research subjects becomes visible. We now discuss some of the possible applications of our work.

Opaque object can be made to focus light with an excellent resolution. Therefore, thin layers of paint might be used as cheap disposable lenses. Scattering can also be used to improve the resolution and, thereby, the data transfer rate of a free space communication system. Instead of creating a focus, multiply scattered light can also be made to form a beam. This could be useful when performing laser spectroscopy of opaque materials. The scattered coherent light could then be collected with a high efficiency, thus greatly improving the sensitivity. Even better, incoherent background light does not form into a beam and, therefore, would not be detected. The wavefront that focuses light through an opaque object is unique for each object. This property could be used in security applications. When, for example, a small photodiode is covered with a layer of white paint, it is only possible to focus light on it when the matching wavefront is known.

Light can also be focused inside an opaque object. The applications of this method are endless. One could, for example, focus light on a single fluorescently labelled cell below the human skin or inside bone. This application would allow one to study, track or destroy individual (malignant) cells. To make this futuristic application possible, the wavefront needs to be constructed faster than the cells in the tissue move. We demonstrated that wavefront shaping in a living flower petal is possible. For wavefront shaping in human tissue, especially in perfused tissue, the speed of the wavefront shaping process needs to improve dramatically. This speed improvement is possible with the use of extremely fast light modulators that have recently been 


\section{6 | Summary and outlook}

developed.

Our method can also be used to focus light inside non-random complex systems. This way, one can focus light on a quantum dot in a three-dimensional photonic crystal cavity, or excite a desired mode in a complex photonic metamaterial or plasmonic device. All in all, wavefront shaping can be used to guide light (or any other wave) to any desired position in an arbitrary complex environment. 


\section{Nederlandse samenvatting}

Dit proefschrift gaat over de voortplanting van licht in ondoorzichtige witte dingen zoals velletjes papier, laagjes verf, eierschillen, bloemblaadjes en tanden. Witte materialen verstoren de voortplanting van licht: als je bijvoorbeeld met een zaklantaarn op een velletje papier schijnt, gaat het licht niet als een bundel door het papier heen, maar in plaats daarvan gloeit het hele papier op met een vaag schijnsel. Het licht is diffuus geworden: het straalt gelijkmatig naar alle richtingen uit en er is niet meer te zien waar het licht oorspronkelijk vandaan kwam. Het is daarom ook onmogelijk om door het materiaal heen te kijken.

Tot nu toe was het niet mogelijk om te beïnvloeden hoe licht zich door een ondoorzichtig materiaal voortplant: het licht werd altijd diffuus. Wij hebben een techniek bedacht waarmee we het licht wél kunnen sturen. Met deze techniek kun je licht door een ondoorzichtige laag heen projecteren of zelfs concentreren op een punt diep binnenin een ondoorzichtig object. Hiermee wordt het misschien ooit mogelijk om licht door huid of bot heen op één enkele gemarkeerde kankercel te focusseren, of om door de ingewikkelde structuur van een microchip heen één enkele transistor te belichten. Ook bij andere toepassingen, zoals telecommunicatie, optische experimenten aan microstructuren en spectroscopie aan poeders kan onze techniek uitkomst bieden. Uiteindelijk zou dit ook kunnen leiden tot een verbetering van bestaande medische beeldvormende technieken.

\section{Interferentie en verstrooiing}

Licht is een golf. Zoals alle golven kunnen lichtgolven met elkaar interfereren. Interferentie is niets anders dan dat twee golven bij elkaar opgeteld worden. Als twee golven tegelijkertijd aankomen zijn ze in fase, zoals te zien is in Figuur 1a. De piek van de ene golf telt dan op bij de piek van de andere golf en het resultaat is een golf met grote pieken en dalen (dit heet constructieve interferentie). Het kan ook zijn dat
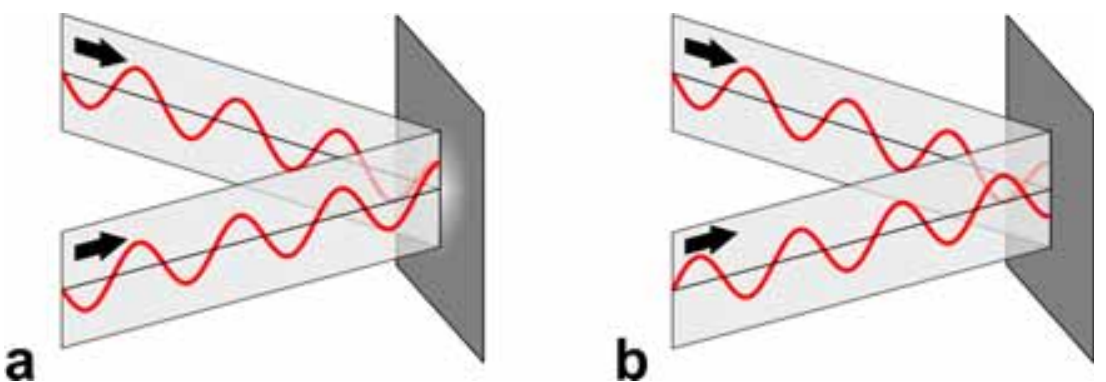

Figuur 1: Interferentie. a) De golven komen tegelijk aan. Ze zijn in fase en versterken elkaar. b) De golven zijn uit fase en doven elkaar uit. 


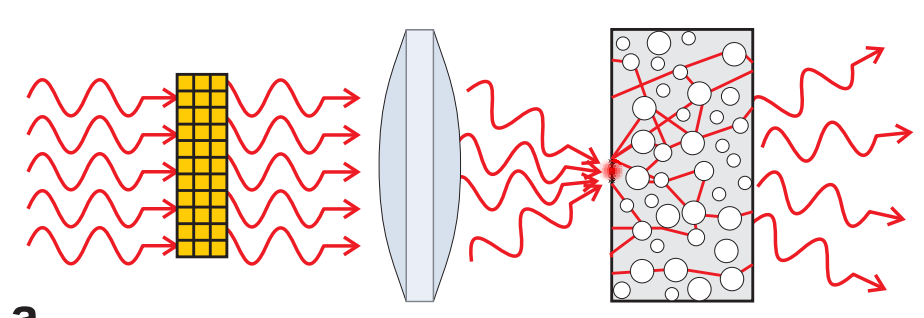

a

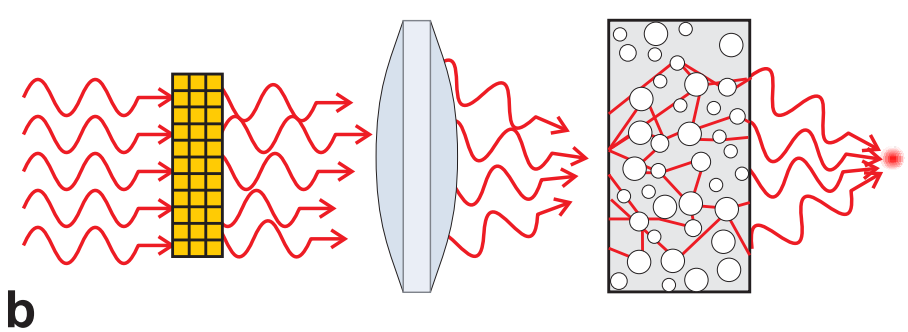

Figuur 2: Principe van het experiment. a) een vlakke golf wordt gefocusseerd op een ondoorzichtig wanordelijk materiaal. Door verstrooiing ten gevolge van microscopische oneffenheden legt het licht een zeer complexe route af. Na honderden keren verstrooien bereikt een klein deel van het licht de achterkant van het materiaal en vormt een diffuse vlek. b) Door de fase van het binnenkomende licht op precies de goede manier aan te passen is het mogelijk om de bijdragen van alle vlakjes constructief te laten interfereren op een punt achter het medium. Het medium werkt in dit geval als een wanordelijke lens en focusseert het licht.

twee golven uit fase zijn. De ene golf komt dan wat later aan dan de andere zodat de pieken van die golf samenvallen met de dalen van de andere golf; het resultaat is dan dat de twee golven elkaar opheffen. Deze situatie is afgebeeld in Figuur $1 \mathrm{~b}$.

In een wit materiaal zitten allemaal kleine deeltjes waar het licht op botst. Bij iedere botsing verandert de voortplantingsrichting van het licht. Het licht 'stuitert' door het materiaal, een beetje zoals het balletje in een flipperkast. Dit proces heet verstrooiing van licht. Al het verstrooide licht interfereert met elkaar, zodat er een complex wanordelijk interferentiepatroon ontstaat. Dit patroon staat bekend als laserspikkel (Engels: speckle). Een voorbeeld van een spikkelpatroon is te zien in Figuur 3.3a in Hoofdstuk 3. Het hangt af van de fase van alle interfererende golven of ergens een heldere of juist een donkere plek ontstaat.

\section{Licht focusseren met een eierschil}

We hebben een opstelling ontworpen die een bundel laserlicht in duizenden vlakjes verdeelt en dan de fase van elk van deze vlakjes afzonderlijk kan aanpassen. Het licht van elk vlakje zal op een andere manier verstrooid worden (zie Figuur 2a). Door de fase van de vlakjes op de juiste manier in te stellen kunnen we nu zorgen dat het verstrooide licht op een bepaalde plek constructief interfeert (Figuur 2b). De lichtintensiteit op die plek neemt daardoor enorm toe, soms wel met een factor duizend. 
Het resultaat is dat een ondoorzichtig object, zoals een eierschil, licht focusseert tot een scherp punt. Eigenlijk werkt de eierschil dus als een 'ondoorzichtige lens'.

Er zijn ongelooflijk veel mogelijke combinaties van fases te maken. Om de juiste combinatie te vinden gebruiken we een computer die de hoeveelheid licht in het gewenste focus meet en de fases aanpast. In het begin is de lichtintensiteit heel laag, maar door de fase van een vlakje aan te passen kunnen we de hoeveelheid licht laten toenemen. De computer herhaalt dit proces voor alle vlakjes. Uiteindelijk hebben we dan een heel ingewikkeld gevormde bundel gemaakt die precies compenseert voor alle botsingen aan de deeltjes in het materiaal. In hoofdstuk 3 laten we zien dat deze methode de optimale combinatie van fases vindt. Omdat deze combinatie afhangt van de positie van alle deeltjes in het materiaal, werkt onze methode nog niet voor materialen waarin de deeltjes bewegen, zoals melk of doorbloed weefsel. Met recent ontwikkelde technologie is het echter mogelijk om het proces zo te versnellen dat we in de toekomst 'real-time' kunnen compenseren voor de bewegende deeltjes.

\section{Belangrijkste resultaten}

Het belangrijkste resultaat van dit proefschrift is dat we in staat zijn om licht door een ondoorzichtig materiaal heen te focusseren. Deze doorbraak heeft nationaal en internationaal veel aandacht gekregen (zie bijvoorbeeld 'Witte verf of eierschaal als brandglas voor bundel laserlicht' in het NRC Handelsblad van 14 juli 2007). Het is niet alleen gelukt om verstrooid licht in een scherp focus samen te laten komen, we begrijpen de resultaten ook kwantitatief. We hebben uitgerekend hoe intens het focus is (Hoofdstuk 3), wat er gebeurt als de deeltjes in het materiaal bewegen (Hoofdstuk 7) en hoe groot het focus is (Hoofdstuk 4). Het blijkt dat het focus van, bijvoorbeeld, een eierschil precies even scherp is als het focus dat je met de best mogelijke lens zou kunnen maken. In Hoofdstuk 6 laten we verder nog zien dat wanordelijke verstrooiing gebruikt kan worden om meer optische informatie te versturen dan normaal mogelijk is.

We zijn ook in staat om licht binnenin een materiaal te focusseren, iets wat zeer interessant is voor bijvoorbeeld medische toepassingen. Dit experiment is technisch veel lastiger dan licht ergens dwars doorheen focusseren. Om de juiste combinatie van fases te vinden is het namelijk nodig om de lichtintensiteit op de gewenste plek te meten. Als je, bijvoorbeeld, binnen in de huid wilt focusseren, is het niet mogelijk om op die plek een detector aan te brengen. We hebben daarom gebruik gemaakt van zeer kleine bolletjes met een fluorescerende verf erin. Dit soort bolletjes zijn wél in te brengen. Door nu te meten hoeveel het bolletje fluoresceert, meten we indirect hoeveel licht er van buiten af op het bolletje valt. Dit experiment staat beschreven in Hoofdstuk 5.

Verstrooiing van licht lijkt wiskundig gezien erg veel op het transport van elektronen in een metaaldraad. We kunnen daarom met licht voorspellingen testen die gedaan zijn voor elektronische systemen. Het voordeel van onze optische methode is dat we het binnenkomende licht heel precies kunnen vormen, iets wat met elektronen nu niet mogelijk is. Eén van de meest verrassende voorspellingen is dat het mogelijk moet zijn om een golf te maken die voor de volle $100 \%$ wordt doorgelaten door een ondoorzichtig materiaal. In Hoofdstuk 8 laten we zien wat dit betekent voor ons 
experiment: als we de binnenkomende lichtbundel optimaliseren om op één punt licht te focusseren moet de intensiteit rondom dat punt ook omhoog gaan. Er zal dus in totaal veel meer licht doorgelaten worden (en er zal dus ook veel minder licht worden gereflecteerd). We hebben in een experiment aangetoond dat de totale transmissie inderdaad significant stijgt (Hoofdstuk 9). Hiermee bevestigen we een belangrijk theoretisch aspect van het transport van elektronen, geluid, licht en andere golven.

\section{Conclusie}

We hebben een methode ontwikkeld om met behulp van interferentie laserlicht door ondoorzichtige materialen heen te sturen. Het op deze manier sturen van laserlicht is een nieuw onderwerp in de optica. Dit proefschrift kan daarom het beste gezien worden als een eerste verkenning van dit gebied. Het is nu al duidelijk dat er nog heel veel verder te experimenteren valt en dat er interessante toepassingen verwacht kunnen worden. We hopen dan ook dat dit nieuwe vakgebied ook de komende jaren nog tot veel spectaculaire resultaten zal leiden. 


\section{Dankwoord}

"Niet in de afzondering zullen we onszelf ontdekken, maar onderweg, in de stad, in de menigte, als ding onder de dingen, als mens onder de mensen."
"Ce n'est pas dans je ne sais quelle retraite que nous nous découvrirons, c'est sur la route, dans la ville, au milieu de la foule, chose parmi les choses, homme parmi les hommes."

- Jean Paul Sartre, Situations (1976)

In geen enkel ander hoofdstuk van mijn proefschrift zul je het woordje 'ik' tegenkomen om de simpele reden dat alles wat ik bereikt heb het resultaat is van een intensieve samenwerking waaraan iedereen op zijn of haar eigen wijze heeft bijgedragen. Daarom wil ik hier iedereen bedanken die mij in de afgelopen vier jaar heeft bijgestaan en heeft gezorgd voor een leuke sfeer in de vakgroep en daarbuiten. Een paar mensen wil ik apart noemen.

Allereerst mijn begeleiders Ad en Allard. Allard, het was jouw idee waar dit project allemaal mee begon. Vanaf het eerste moment was ik enthousiast en je hebt dat enthousiasme steeds aangewakkerd met de vele (soms wilde) ideeën die in onze gesprekken ontstonden. Ik vond het heel prettig om met je samen te werken en soms ook over andere dingen dan natuurkunde te kunnen praten.

Ad, het is mede dankzij jouw flexibele instelling dat we uiteindelijk met zijn allen dit mooie resultaat hebben behaald. Ik bewonder je scherpe inzicht in de natuurkunde en je visie op hoe goede wetenschap hoort te werken. Ik denk met plezier terug aan mijn bezoeken aan het Amolf en de momenten dat we over een zin gebogen zaten om nét dat ene woord te vinden dat precies de goede lading had.

$\mathrm{Na}$ de verhuizing bleef in Enschede een kleine, maar gezellige groep over. Bas, bedankt voor je snelle hulp met computers. Cock, dank je voor al het werk dat je hebt verricht aan mijn opstelling. Je hebt altijd voor een goede sfeer in de groep gezorgd en ik ben blij je als kamergenoot gehad te hebben. Hannie, mijn meest nieuwschierige kamergenoot en groepspsychologe. Karen Munnink stond altijd klaar voor hulp met formulieren en andere dingen waar ik niets van snap. Léon de masterchief van het chemisch lab en daarbuiten. Phil, blijf zwemmen! Raymond, het was altijd een prettige verrassing als je weer wat leven in de brouwerij kwam brengen. Willem Tjerkstra, de ideale schrijver om mee naar de film te gaan. En natuurlijk Willem Vos die als een soort vliegende keep zowel in Amsterdam als in Enschede de vakgroepen bestuurt. Willem, dank je voor je ondersteuning, inbreng en enthousiasme.

Wouter en Elbert, het was erg leuk en leerzaam om jullie te mogen begeleiden. Jullie grote inzet en creatieve ideeën hebben veel voor mij betekend. Elbert heeft in grote mate bijgedragen aan Hoofdstukken 2 en 5, hartelijk dank hiervoor.

Het was altijd gezellig om op het AMOLF langs te komen. In het bijzonder herinner ik me de vrolijke kamer met Bart, Merel en Ramy; Alex, die mij heeft geïnspireerd om weer Sartre gaan lezen en Bernard, het is altijd een plezier met je te praten, ook al kan ik je af en toe niet helemaal bijhouden. Otto, Paulo, Patrick, Sanli bedankt voor de inspirerende discussies. Verder wil ik iedereen in de Photon Scattering en Photonic Bandgaps groepen bedanken voor de prettige samenwerking. Ook veel dank aan de 
Photon Scattering groep voor het uitlenen van de $\mathrm{TiO}_{2}$ samples en aan Simone voor het uitlenen van haar melktand.

Ook met sommige oud groepsleden heb ik een hechte band opgebouwd. Karen en Tom, jullie waren geweldige collega's, zeker ook toen de dingen tegenzaten. Peter, Boris, Martijn, jullie hebben me wegwijs gemaakt in de natuurkunde. Tijmen, op de één of andere manier komen we altijd op bijzondere plaatsen terecht... Femius, Ivan en Nina, Lydia, Professor Valentin Freilikher, en alle andere geweldige mensen bij COPS, dankjewel!

I would like to thank Professor Sir John Pendry and Professor Vinod Subramaniam for their enthusiastic response to our research.

Ik heb de afgelopen vier jaar niet alleen maar werkend doorgebracht. Ik wil al mijn vrienden bedanken die er voor hebben gezorgd dat ik af en toe het laboratorium uit kwam om de echte wereld te (her) ontdekken. in het bijzonder Claartje, Kell, Marieke en Maarten, Mieke en Willem Mees, Moes, Nienke en Pieter, Wietse en alle mensen van de Parkweg. Frans, met jou hardlopen was altijd erg ontspannend en gezellig.

Thomas, je woont ver weg, maar je bent dichtbij. Je hebt me enorm geholpen met alles wat me de afgelopen jaren bezig gehouden heeft. Dank je. ¡Estíbaliz, cuida bien de el! Henrik, Marc, ik ben blij jullie als paranimfen te hebben. Ik zal proberen niet flauw te vallen, maar het stelt me gerust dat er twee goede vrienden (met veel toneelervaring) klaar staan om het over te nemen. Iris, je hebt veel voor me betekend de afgelopen jaren. Ik hoop dat jij ook heel veel plezier zult beleven aan je onderzoek.

Papa, Mama, mijn hoofd duizelt als ik bedenk wat er in de afgelopen jaren allemaal is gebeurd. Al die tijd hebben jullie mij gesteund en waren jullie enthousiast en geïnteresseerd in mijn onderzoek. Dankjewel. Ik ben blij jullie te zien genieten van jullie reizen en van de kleine en grote dingen in het leven. Casper en Karin, het is goed om familie te hebben die je steunt en die ook weet hoe ze een goed feest moeten organiseren, bedankt voor de leuke tijden!

Bert, je bent er helaas niet meer, maar jouw aanstekelijke enthousiasme voor de wetenschap heeft mij voor altijd gevormd. Dank je wel hiervoor. 

